\title{
Advancing ReBCO-CORC Wire and Cable-in-Conduit Conductor Technology for Superconducting Magnets
}

\author{
Tim Mulder
}





\section{ADVANCING REBCO-CORC WIRE AND \\ CABLE-IN-CONDUIT CONDUCTOR TECHNOLOGY FOR SUPERCONDUCTING MAGNETS}

Tim Mulder 


\section{Dissertation Graduation Committee:}

Chairman: Prof. dr. ir. J. W. M. Hilgenkamp University of Twente

Promotor: $\quad$ Prof. dr. ir. H. H. J. ten Kate

University of Twente

Co-Promotor: Dr. M. M. J. Dhallé

University of Twente

Members: $\quad$ Prof. dr. ir. H. J. M. ter Brake

University of Twente

Prof. dr. ir. R. Akkerman

University of Twente

Prof. dr. E. Lomonova

Prof. dr. R. Ross

University of Eindhoven

Dr. ir. A. Kario

University of Delft

Dr. ir. D.C. van der Laan

Karlsruhe Institute of Technology

University of Colorado / ACT

The work in this thesis was carried out at CERN, Experimental Physics Department, ATLAS Magnet Group, Geneva, Switzerland; and supported by the University of Twente, Faculty of Science and Technology, chair of Industrial Application of Superconductivity and chair Energy-Materials-Systems, Enschede, the Netherlands.

Publisher:

Tim Mulder, University of Twente,

P.O. Box 217, 7500 AE Enschede, the Netherlands

(C) Tim Mulder, Enschede, the Netherlands - 2018

No part of this work may be reproduced by print photocopy or any other means without the permission in writing from the publisher.

Printed by Ipskamp Printing, Enschede, the Netherlands

ISBN: 978-90-365-4616-4 


\section{ADVANCING REBCO-CORC WIRE AND \\ CABLE-IN-CONDUIT CONDUCTOR TECHNOLOGY \\ FOR SUPERCONDUCTING MAGNETS}

\section{DISSERTATION}

to obtain

the degree of doctor at the University of Twente, on the authority of the rector magnificus, prof. dr. T.T.M. Palstra,

on account of the decision of the graduation committee, to be publicly defended on Friday September 28, 2018 at 12:45

by

\section{Tim Mulder}

born on July 6, 1989

in Doetinchem, the Netherlands 
This thesis has been approved by the promotor:

Prof. dr. ir. H. H. J. ten Kate 


\section{Contents}

1 Introduction 1

1.1 Superconductors for Large Magnet Systems . . . . . . . . . . . . . . 2

1.1.1 Low Temperature Superconductors (LTS) . . . . . . . . . . . 2

1.1.2 High Temperature Superconductors (HTS) . . . . . . . . . . . 3

1.2 ReBCO Multi-Tape Cables . . . . . . . . . . . . . . . . . 4

1.2 .1 Roebel Cable . . . . . . . . . . . . . . . . . . 4

1.2.2 Twisted Stacked Tape Cable (TSTC) $\ldots \ldots \ldots \ldots \ldots$

1.2.3 Conductor On Round Core (CORC) . . . . . . . . . . . . . 6

1.3 Joints for ReBCO CORC Conductors . . . . . . . . . . . . . . . . . . 8

1.4 Scope and Objective of the Thesis . . . . . . . . . . . . . . . . . 9

2 From ReBCO Coated Conductor to Conductor On Round Core 11

2.1 Introduction . . . . . . . . . . . . . . . . . . 11

2.2 ReBCO Coated Conductor Technology . . . . . . . . . . . . . . . . 12

2.2.1 Tape Performance and Recent Advancements . . . . . . . . . . 14

2.2 .2 Strain and Tape Bending . . . . . . . . . . . . . . . . 18

2.3 CORC Conductor Geometry, Bending and Challenges . . . . . . . . . . . 21

2.3 .1 Geometry of CORC . . . . . . . . . . . . . . . . . 21

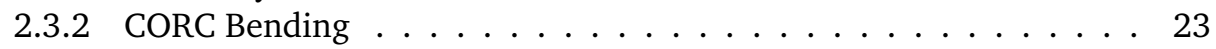

2.3 .3 Production Process . . . . . . . . . . . . . . . . 25

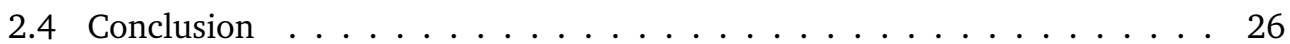

3 Current Distribution in and Resistance of CORC Joints 27

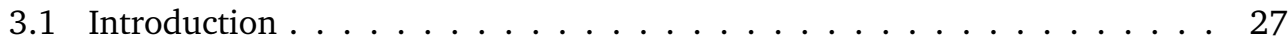

3.2 Physics Aspects of Current Sharing . . . . . . . . . . . . . . . 30

3.2.1 Characteristic Tape Contact Resistance . . . . . . . . . . . . . 31

3.2.2 Current Sharing at Constant and Alternating Current . . . . . . . 32

3.2.3 Current Sharing in HTS Power Cables . . . . . . . . . . . . . 40

3.3 Novel Joint Design . . . . . . . . . . . . . . . . . . . . . . . . . . 42

3.3.1 New Concept . . . . . . . . . . . . . . . . . . . 42

3.3.2 Nodal-Network Joint Model . . . . . . . . . . . . . . . . . . . . 43

3.3.3 Calculation of Current Distribution . . . . . . . . . . . . . 45

3.3.4 Optimization of the Stage Length . . . . . . . . . . . . . . 47

3.3.5 Calculation of Joint Resistance . . . . . . . . . . . . . . 48 
3.3.6 Parametric Sensitivity Analyses . . . . . . . . . . . . . . 50

3.4 Joints for CORC Cable-In-Conduit Conductors . . . . . . . . . . . . . . . 52

3.4.1 Optimizing the Current Distribution Between CORC Strands . . . . 53

3.4 .2 Summary of the Modeling Results . . . . . . . . . . . . . 56

3.4 .3 Engineering Values . . . . . . . . . . . . . . . . . . 57

3.5 Experimental Realization of the Novel CORC Joints . . . . . . . . . . . . . 58

3.5.1 Preparation of CORC Conductor Ends . . . . . . . . . . . . 58

3.5.2 Terminals for Single CORC Conductors . . . . . . . . . . . . 58

3.5.3 Terminals for CORC Cable-In-Conduit Conductors . . . . . . . . . . 61

3.6 Experimental Realization of CORC Joint Terminals . . . . . . . . . . . . . 64

3.7 Conclusion . . . . . . . . . . . . . . . . . . . . 64

4 CORC Wires and Cables $\quad 65$

4.1 Introduction . . . . . . . . . . . . . . . . 65

4.2 Predicting the Performance of CORC . . . . . . . . . . . . . 66

4.2 .1 Geometrical Scaling . . . . . . . . . . . . . . . . 66

4.2.2 Scaling with Temperature and Magnetic Field . . . . . . . . . . . 67

4.2.3 Fitting Equation for ReBCO Based Conductors . . . . . . . . . 70

4.3 Performance of a Single CORC Cable . . . . . . . . . . . . . . . . 71

4.3.1 CORC Sample and Test Setup . . . . . . . . . . . . . . . 72

4.3.2 Performance of the CORC Cable and its ReBCO Tapes . . . . . . . . 74

4.3.3 Performance of the Joint Terminals . . . . . . . . . . . . . . . 76

4.3.4 Quench Behavior and Conductor Survival . . . . . . . . . . . 76

4.3.5 Discussion and Conclusion Regarding the CORC Cable Test . . . . . 79

4.4 Test of CORC Wires in Coil Configuration . . . . . . . . . . . . . . . . . 80

4.4 .1 CORC Wire Samples . . . . . . . . . . . . . . . . . 82

4.4.2 Degradation of the First Sample . . . . . . . . . . . . . . . 84

4.4.3 Electrical Performance of the CORC Wires . . . . . . . . . . . . . 84

4.4.4 Effect of Electro-Magnetic Load Cycling . . . . . . . . . . . . . . 85

4.4 .5 Analyses of Extracted Tapes . . . . . . . . . . . . . . . . . 85

4.4.6 Effect of Temperature on Wire Performance $\ldots \ldots \ldots \ldots$

4.4 .7 Joints for CORC wires . . . . . . . . . . . . . . . . . 88

4.4 .8 Conclusion . . . . . . . . . . . . . . . . . . 91

4.5 ReBCO CORC Demonstration Magnets . . . . . . . . . . . . . . 91

4.5.1 Current Status of ReBCO Coated Conductor based Magnets . . . 92

4.5.2 CORC Racetrack Coil at CERN . . . . . . . . . . . . . . . . . . . 93

4.5 .3 CORC Wire for the Racetrack Coil . . . . . . . . . . . . . . . . 94

4.5.4 Winding and Test of the CORC Racetrack Demonstrator . . . . . 96

4.6 Conclusion . . . . . . . . . . . . . . . . . . . . 99

5 Six-Around-One CORC Cable-In-Conduit Conductors 101

5.1 Introduction . . . . . . . . . . . . . . . . . . . . . . . . 101

5.2 Design of CORC type Cable-In-Conduit Conductors . . . . . . . . . . . 104

5.2 .1 CORC CICC Layout . . . . . . . . . . . . . . . . . . . . . 104

5.2.2 Realization of an Exercise Model CORC CICC . . . . . . . . . . 105

5.3 Worlds First CORC type Cable-In-Conduit Conductor . . . . . . . . . . 106 
5.3.1 Design and Properties . . . . . . . . . . . . . . . 106

5.3.2 Preparation of the CICC and the Sample Holder . . . . . . . . 108

5.3.3 In-field Performance at Liquid Helium Temperature . . . . . . . . 111

5.3.4 Self-Field Performance at $77 \mathrm{~K} \ldots \ldots \ldots \ldots$

5.3.5 Performance of the Joint Terminals at $77 \mathrm{~K} \ldots \ldots \ldots \ldots \ldots$

5.3 .6 Discussion . . . . . . . . . . . . . . . . . . . . 114

5.3 .7 Conclusion . . . . . . . . . . . . . . . . . . . . 116

5.4 Two New CORC CICC Samples for Magnets aiming at Fusion Experiments and Particle Detectors . . . . . . . . . . . . . . . . 117

5.4.1 Layout of the Two CORC Six-Around-One CICCs . . . . . . . . . 117

5.4.2 Jacketing of the CORC Cable-In-Conduit Conductors . . . . . . . 120

5.4.3 Joint Terminal Layout and Optimization . . . . . . . . . . . . 122

5.4.4 Test Setup of the Two CORC CICCs . . . . . . . . . . . . . . 123

5.4.5 Results of the Two CORC Cable-In-Conduit Conductors . . . . . . . 123

5.4.6 Extracted Single-Strand and Single Tape Analyses . . . . . . . . . 135

5.4.7 Plausible Cause of the Observed Degradation . . . . . . . . . . 139

5.4.8 Discussion on Future Conductor and Joint Improvements . . . . . 140

5.4 .9 Conclusion . . . . . . . . . . . . . . . . . . . 140

5.5 CORC CICC Bus Bars . . . . . . . . . . . . . . . . . . . . . . . 142

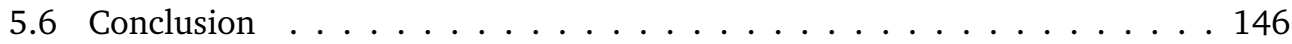

6 Conclusion 147

6.1 CORC Wires and Cables for High-Field Magnets . . . . . . . . . . . . 147

6.2 CORC Cable-In-Conduit Conductors for Large-Scale Magnets . . . . . . . . 149

6.3 Novel Joint Terminals for CORC Conductors . . . . . . . . . . . . . . 150

$\begin{array}{ll}\text { Summary } & 163\end{array}$

$\begin{array}{ll}\text { Samenvatting } & 167\end{array}$

$\begin{array}{ll}\text { Acknowledgments } & 171\end{array}$

$\begin{array}{ll}\text { List of Publications } & 173\end{array}$ 



\section{Chapter 1}

\section{Introduction}

ReBCO Coated Conductors are ceramic flat tape High Temperature Superconductors (HTS) capable of carrying large currents in a broad temperature and magnetic field range. ReBCO tapes significantly exceed the performance reach of $\mathrm{NbTi}$ and $\mathrm{Nb}_{3} \mathrm{Sn}$ Low Temperature Superconductors (LTS), which allows the creation of large-scale magnets capable of generating magnetic field far beyond $20 \mathrm{~T}$ at $4.5 \mathrm{~K}$ and of magnets operating in the temperature range of 30 to $60 \mathrm{~K}$, a range served uniquely by ReBCO material.

Large-scale magnets require currents far beyond the capacity of a single ReBCO tape and therefore multiple ReBCO tapes must be combined into a multi-tape, high-current ReBCO cable. The three main cabling concepts today are the Roebel, Twisted Stack Tape and Conductor On Round Core (CORC) cable. The focus of the work presented in this thesis is on the development of CORC technology. CORC type conductors can be grouped in three categories: CORC cable, which is a standalone general purpose HTS cable; thin CORC wire, that aims at a high-current density for application in accelerator magnets and insert coils; and CORC Cable-In-Conduit Conductor designed for enabling stable, high-current magnets and their bus lines. A key aspect of this work is the development of joint terminals for CORC conductors that allow low-resistive and homogeneous current injection and extraction. This logical line through the work is also summarized in Figure 1.1

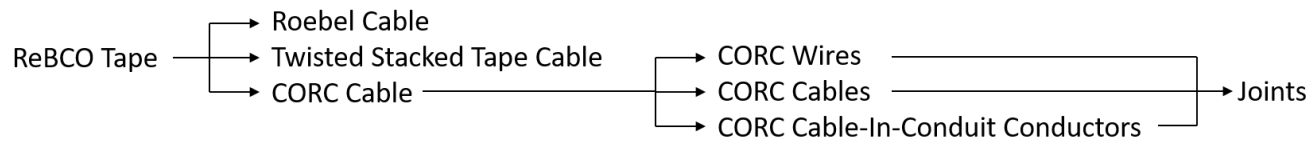

Figure 1.1: The connecting line showing the path from the ReBCO tape to the Conductor On Round Core (CORC) wires, cables and Cable-In-Conduit Conductors and their joint terminals. 


\subsection{Superconductors for Large Magnet Systems}

\subsubsection{Low Temperature Superconductors (LTS)}

A superconducting material loses electrical resistivity when operated at a sufficiently low combination of temperature $T$, current density $J$ and magnetic field $B$. Cables manufactured from such a material are able to carry orders of magnitude larger transport currents than normal conductors of similar size, i.e. copper or aluminium, since superconductors do not experience ohmic loss. Consequently, used in a magnet they are able to generate a much higher magnetic field with less conductor material. The two mostly used superconducting materials today are Niobium-Titanium (NbTi) [1] and Niobium-Tin $\left(\mathrm{Nb}_{3} \mathrm{Sn}\right)[2,3]$. These materials are considered Low Temperature Superconductors (LTS), which have a critical temperature $T_{c}$ below $20 \mathrm{~K}$. NbTi has a $T_{c}$ of $9.3 \mathrm{~K}$, while $\mathrm{Nb}_{3} \mathrm{Sn}$ has a $T_{c}$ of $18.3 \mathrm{~K}$. In practice, both are operated only at temperatures of $1.9 \mathrm{~K}$ (in superfluid helium) or of about $4.5 \mathrm{~K}$, because their performance declines steeply by going up in temperature. NbTi-based conductors are practical in production and handling and are relatively inexpensive. $\mathrm{Nb}_{3} \mathrm{Sn}$ conductors offer a performance in terms of achievable $T, J$ or $B$, which is almost double of $\mathrm{NbTi}$, but require a more difficult production process since such conductors need a precise heat treatment after coil winding. Moreover, they are less practical in handling, since $\mathrm{Nb}_{3} \mathrm{Sn}$ becomes brittle after heat treatment. Conductors of both types of material are well-developed and implemented in many small and large scale magnet applications today.

A major, well-known application of LTS is the magnet system of the Large Hadron Collider (LHC) at CERN used for particle bending. CERN's LHC comprises around $120015 \mathrm{~m}$ long NbTi dipole magnets, $400 \mathrm{NbTi}$ quadrupole magnets, three large NbTi-based detector magnet systems and a further few thousand small NbTi based corrector- and other magnets. The operating currents of these magnets range from a few hundred ampere to 21 $\mathrm{kA}$, with magnetic fields ranging from a few $\mathrm{mT}$ to $9 \mathrm{~T}[4,5,6,7]$.

Another well-known application of LTS materials is nuclear fusion, like in the ITER project, which aims to demonstrate the principle of electric power generation using nuclear fusion. ITER mostly uses $\mathrm{Nb}_{3} \mathrm{Sn}$-based magnets to generate dynamic magnetic fields peaking in the range of 12 to $13 \mathrm{~T}$ to contain and shape the plasma and to generate the large currents that drive the fusion process. $\mathrm{Nb}_{3} \mathrm{Sn}$-based conductors are required for most ITER coils since the magnetic fields involved stretch far beyond the limits achievable with NbTi-based conductors [8].

A relatively new material, often classified outside the LTS temperature region, is the alloy Magnesium-Boride $\left(\mathrm{MgB}_{2}\right)$ with a $T_{c}$ value of $39 \mathrm{~K}$. The production of $\mathrm{MgB}_{2}$ cables requires a heat treatment similar to the one needed for $\mathrm{Nb}_{3} \mathrm{Sn}$ cables and the cables are also brittle after heat treatment. The high magnetic field performance of $\mathrm{MgB}_{2}$ cables at $4.2 \mathrm{~K}$ is worse than niobium-based cables as the conductor's critical current density is reduced steeply by introducing an external magnetic field and therefore these type of cables are mainly designed for superconducting bus lines for transport of high currents [9], 
but also for conduction-cooled MRI magnets with operating temperatures in the range of 20-25 K (to avoid the use of expensive and lot less accessible helium as coolant).

\subsubsection{High Temperature Superconductors (HTS)}

A further increase in magnetic field or operating temperature can be achieved only with High Temperature Superconductors (HTS). HTS materials typically have critical temperatures above $77 \mathrm{~K}$ and therefore can be cooled with for example liquid nitrogen or helium gas. The mostly developed HTS materials are the ceramics $\operatorname{ReBCO}[10]\left(R e \mathrm{Ba}_{2} \mathrm{Cu}_{3} \mathrm{O}_{x}\right.$ with $R e=$ Rare Earth, commonly either yttrium or gadolinium) and BSCCO [11] $\left(\mathrm{Bi}_{2} \mathrm{Sr}_{2}\right.$ $\mathrm{Ca}_{n-1} \mathrm{Cu}_{n} \mathrm{O}_{2 n+4+x}$ ), where Bi-2223 (tapes) and Bi-2212 (round wires) are the most common compositions. As discussed in more detail in Chapter 2, ReBCO conductors are produced in the shape of a flat tape with usually a 1-2 $\mu \mathrm{m}$ thin superconducting layer coated on a metallic (steel or Hastelloy) substrate via chemical- or laser deposition. Therefore it is called a ReBCO Coated Conductor (CC). BSCCO is produced as tape or round wire depending on the detailed composition of the BSCCO compound, but BSCCO conductors are becoming obsolete as the ReBCO Coated Conductor is highly preferred over BSCCO due to its better in-field performance at temperatures above $30 \mathrm{~K}[12,13]$. Table 1.1 provides an overview of the basic properties of the six most-used superconductors for magnet technology.

Table 1.1: Key properties of the six most common superconducting materials for magnet systems.

\begin{tabular}{lcccc}
\hline \hline & $\begin{array}{c}\text { Strand Shape } \\
{[-]}\end{array}$ & $\begin{array}{c}\mathrm{T}_{c}(0 T, 0 A) \\
{[\mathrm{K}]}\end{array}$ & $\begin{array}{c}\mathrm{B}_{c 2}(0 K, 0 A) \\
{[\mathrm{T}]}\end{array}$ & $\begin{array}{c}\text { Brittle } \\
{[-]}\end{array}$ \\
\hline $\mathrm{NbTi}$ & Round/Rect. Wire & 9.3 & 14.6 & No \\
$\mathrm{Nb}_{3} \mathrm{Sn}$ & Round/Rect. Wire & 18.3 & $24-28$ & Yes \\
$\mathrm{MgB}_{2}$ & Round/Rect. Wire & 39 & 16 & Yes \\
$\operatorname{ReBCO}$ & Flat Tape & 93 & $>100$ & Slightly \\
$\mathrm{Bi}-2212$ & Round Wire & 85 & $>100$ & Yes \\
$\mathrm{Bi}-2223$ & Flat Tape & 110 & $>100$ & Yes \\
\hline \hline
\end{tabular}

Although HTS materials can be operated at a much higher temperature than LTS materials, HTS really excel in performance at low temperatures due to their ability to retain a high critical current density in relatively high magnetic fields, due to their upper critical magnetic field of over $100 \mathrm{~T}$ at $5 \mathrm{~K}$ [14]. In practice, NbTi magnets are capable of generating magnetic field up to $9 \mathrm{~T}$ and $\mathrm{Nb}_{3} \mathrm{Sn}$ magnets go up to about $20 \mathrm{~T}$. HTS ReBCO-based magnets have the potential of generating magnetic fields far beyond $20 \mathrm{~T}$, as already demonstrated at the NHMFL with a 32 T LTS/HTS hybrid solenoid [15]. Such an increase in magnetic field will benefit many applications, e.g. circular particle colliders that use magnetic field for particle bending. A higher magnetic field allows for higher particle energy and consequently for higher collision energies, which may lead to exciting new discoveries in particle physics.

Another important benefit of HTS materials is their much higher electrical and thermal stability compared to LTS [16]. Around $5 \mathrm{~K}$, the critical current and critical field of an HTS 
conductor depend only weakly on temperature, compared to LTS materials where the temperature is a very important factor in the performance. Consequently, a small amount of energy deposited in an LTS cable operating near full capacity easily causes an abrupt change from the superconducting to the normal state (quench), while energies of one to several orders of magnitude larger are required for a similar effect on an HTS cable. Conductor stability is a very important aspect of large magnet systems with a stored energy of many GJ, since a conductor quench would result in a considerable energy dump within the cold-mass of the magnet system, resulting in a long down-time due to discharching, re-cooling and re-charging of the magnets. The combination of enhanced stability and performance at temperatures around or below $5 \mathrm{~K}$ are also important for standalone magnets for scientific purpose, often outweighing any additional cooling costs.

The operating temperature range of 30 to $60 \mathrm{~K}$ is unique to (ReBCO) HTS. Magnet operation in this temperature range allows to cool the conductors with helium gas or by direct conduction when coupled to cooling machines, which offers a simplification of the cryogenic system and operating costs compared to conductors cooled by liquid helium. HTS materials also allow for superconducting power transmission in the electrical grid, as liquid nitrogen cooled ReBCO-based transmission cables are well underway to become economically viable for solving specific bottlenecks compared to the present copper transmission cables $[17,18]$.

\subsection{ReBCO Multi-Tape Cables}

The performance of ReBCO tape conductors has sky-rocketed in the last decade due to major technological improvements of the conductor production process. There are many ReBCO tape manufacturers today, together producing a wide range of tapes varying in width, thickness and current carrying capacity. The standard ReBCO tapes of most manufacturers are 4 and $12 \mathrm{~mm}$ in width, have a Hastelloy substrate thickness of $50 \mu \mathrm{m}$ or $100 \mu \mathrm{m}$ and carry 30 to $40 \mathrm{~A} / \mathrm{mm}$ width at $77 \mathrm{~K}$, which increases to $60-120 \mathrm{~A} / \mathrm{mm}$ at $4.2 \mathrm{~K}$ and $10 \mathrm{~T}$, strongly depending on the manufacturer and on tape type. Still, largescale magnets often require currents well beyond the capacity of a single ReBCO tape and therefore multiple tapes have to be combined into a multi-tape, high-current ReBCO cable. Having multiple strands within a single cable also improves cable stability in case of small defects in single strands, as well as it reduces inductive and coupling losses caused by dynamic currents and magnetic fields. The focus in HTS cable development is mainly on three different designs: the Roebel cable [19, 20], the Twisted Stacked Tape Cable [21] and the Conductor On Round Core cable [22, 23]. Each multi-tape cable has its own advantages and target application, which is discussed in more detail below.

\subsubsection{Roebel Cable}

The ReBCO Roebel cable is based on a century old cabling concept named after Ludwig Roebel [24] designed to reduce AC loss in copper bus bars. The concept was proposed for a superconducting context in 1999 in a paper by Martin Wilson, who suggested to 
prepare Roebel conductors from HTS tapes [25] and the first ReBCO Roebel cable was prepared at the Karlsruhe Institute of Technology in 2006 [26]. A Roebel cable comprises several meander-shaped ReBCO tapes woven together as shown in Figure 1.2. ReBCO tapes in the Roebel cable start as $12 \mathrm{~mm}$ wide tapes and the meandering shape is created by cutting out parts of the tape width using a reel-to-reel punching machine [19].

The advantage of the Roebel cable is its ribbon shape, allowing a high superconductor to stabilizer ratio, in combination with fully transposed ReBCO tapes, which allows a high cable current density with limited AC loss. The cable is flexible in the 'out-of-plane' bending direction, which enables relatively straightforward coil winding comparable to using NbTi based Rutherford cables. On the other hand, the more difficult 'in-plane' bending complicates its use in more complex coil shapes. The Roebel cable is developed primarily for future generations of fully HTS or hybrid accelerator magnets, racetracktype motor and generator coils and high-field insert coils. The development of Roebel cables is already in an advanced stage and the first Roebel-based demonstration magnets are under construction [27].

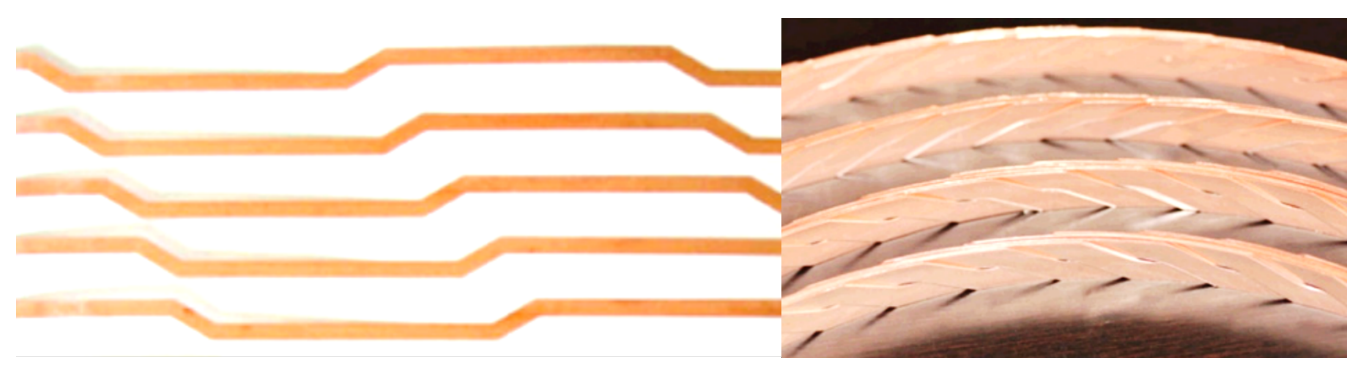

Figure 1.2: Picture of the meandering ReBCO tapes used in Roebel cables and the final woven conductor [19].

\subsubsection{Twisted Stacked Tape Cable (TSTC)}

The Twisted Stacked Tape Cable (TSTC) was introduced by Takayasu et al. in 2011 and comprises a stack of ReBCO coated conductors with a long twist [21], as presented in Figure 1.3. The TSTC aims mainly for application in high-field magnets, but also for implementation in the power transport sector. The TSTC has a high current density, but lacks flexibility, since a full stack of tapes is difficult to bend. Twisting of the stacked tapes can only be achieved with relatively long pitch lengths and therefore such conductor is mainly applicable for DC or quasi-DC applications. The bare shape of the twisted cable is unfit for magnet winding, therefore such cable is often placed within a round copper shell or within a groove of a larger central former to create axial symmetry, which allows magnet winding but obviously also reduces the conductor's current density, as shown in Figure 1.3. The development of the TSTC has focused in recent years mainly on TSTCbased Cable-In-Conduit Conductors aiming at high current for large high-field magnets in nuclear fusion experiments [28]. 


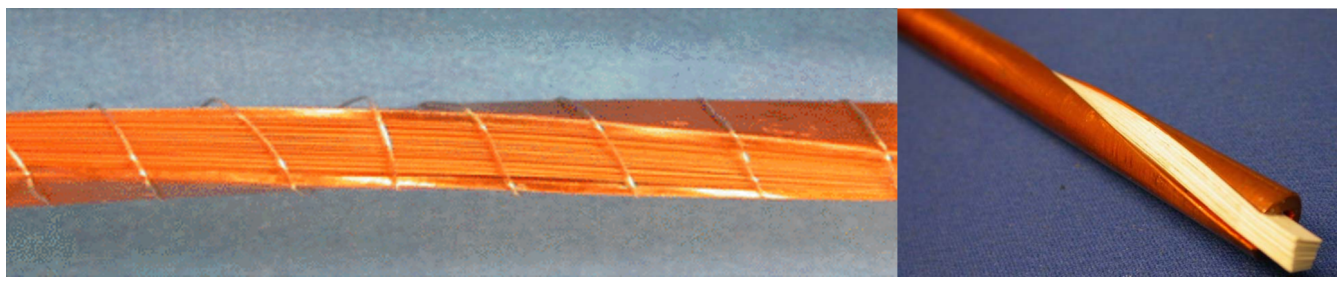

Figure 1.3: Picture of the bare Twisted Stacked Tape Conductor [21] and a round conductor comprising a TSTC encapsulated in a copper shell [28].

\subsubsection{Conductor On Round Core (CORC)}

The Conductor On Round Core (CORC) conductor, the main topic of this thesis, was introduced by D. van der Laan in 2009 [22, 23] and is currently commercially available through the company Advanced Conductor Technologies (ACT). CORC features a round cable shape, with the ReBCO tapes twisted in many layers around a central metallic core to limit AC loss. The CORC conductor, as presented in Figure 1.4, differentiates itself from the Roebel and Twisted Stacked type conductors by offering a high omni-directional flexibility, which allows for straightforward magnet winding, and by the conductor's round symmetric shape that provides a high resiliency towards transverse loads. This all comes at the cost of a lower superconductor to stabilizer ratio and thus a lower overall current density than in the Roebel- and Twisted Stacked Tape cables, due to the presence of the metallic core. In contrast to the Roebel conductor, no tape material is lost in the production process by cutting. Although, the CORC conductor has 30\% higher tape use than the TSTC due to the tape spiraling. The production process of CORC wires and cables is automated and extra features, e.g. polyamide insulation, can directly be incorporated in the production process, which allows for direct of-the-shelf use similar to thick NbTi wires.

CORC-type conductors can be categorized in three different groups, each with its own target application, advantages and disadvantages:

- The CORC cable is a CORC conductor with a typical diameter between 5 and $8 \mathrm{~mm}$, with 3 or $4 \mathrm{~mm}$ wide ReBCO tapes, which can be used standalone or as a strand for the CORC Cable-In-Conduit Conductor discussed below. The mean current density of such a CORC cable is approximately $150 \mathrm{~A} / \mathrm{mm}^{2}$ at $4.2 \mathrm{~K}$ and $20 \mathrm{~T}$, which is obviously lower than the current density in Roebel or Twisted Stacked cables due to the conductor's core. Therefore the CORC cable's target application areas are superconducting magnets in which stability is key and power transmission in confined spaces (such as in navel vessels).

- The CORC wire is a thin CORC conductor with an outer diameter smaller than $4 \mathrm{~mm}$ [29]. These wires are developed for higher flexibility with smaller bending radii, as low as $20 \mathrm{~mm}$, compared to the CORC cable and are aimed at application in accelerator magnets, high-field insert coils or standalone solenoids for general purpose. The CORC wire uses the latest state-of-the-art $2 \mathrm{~mm}$ wide $R e B C O$ tapes with a substrate thickness of $30 \mu \mathrm{m}$. Current densities of over $300 \mathrm{~A} / \mathrm{mm}^{2}$ at $4.2 \mathrm{~K}$ and 
$20 \mathrm{~T}$ can be reached due to the wire's thin core and the high-performance ReBCO tapes. A picture of a CORC cable and a CORC wire is presented in Figures 1.4a and $1.4 \mathrm{~b}$.

- The CORC Cable-In-Conduit Conductor (CORC CICC) comprises a cable composed of multiple bundled CORC strands, jacketed by a metal shell [30]. The CORC CICC is developed for high current, high-field magnets, where stability is a key requirement, but also for HTS bus bars that feed current to such large magnet systems. CORC CIC-Conductors are typically designed to carry over $50 \mathrm{kA}$ at $10 \mathrm{~T}$ at a temperature of $4.5 \mathrm{~K}$, with moderate current densities below $150 \mathrm{~A} / \mathrm{mm}^{2}$. A picture of a bundled six-around-one CORC Cable-In-Conduit Conductor is presented in Figure 1.4c.

(a)

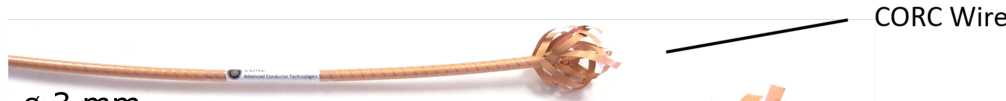

$\varnothing 3 \mathrm{~mm}$

(b)

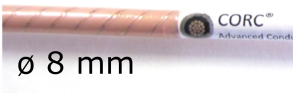

(c)

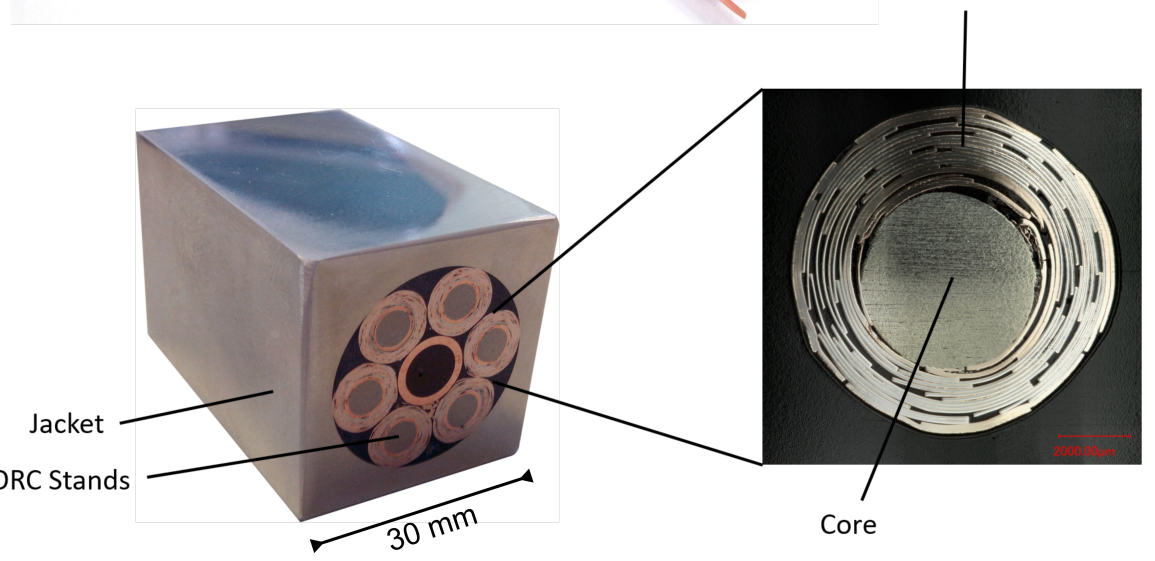

Figure 1.4: Picture of a thin flexible CORC wire and a thicker CORC cable/strand (courtesy of D. van der Laan) and of a multi-strand Cable-In-Conduit Conductor.

Table 1.2 provides characteristic operating currents and current densities of conductors used in existing superconducting magnets and bus bars, as well as the corresponding values for $R e B C O$ cables, which are currently under development. The table demonstrates that the ReBCO-based conductors under development are able to carry currents that exceed the performance of the well-developed LTS conductors. Further research on ReBCO coated conductors and multi-tape ReBCO cables will lead to a further increase in their current-carrying capacity and thereby boost the performance of future superconducting magnets, bus bars and other high current applications even further. 
Table 1.2: Rated performance of practical conductor examples of various material types in existing applications and examples of ReBCO based conductors that are currently under development.

\begin{tabular}{|c|c|c|c|c|c|}
\hline & $\begin{array}{c}\text { Material } \\
{[-]}\end{array}$ & $\begin{array}{l}\text { Dimensions } \\
{[\mathrm{mm} \times \mathrm{mm}]}\end{array}$ & $\begin{array}{c}\mathrm{I}_{o p}(B, T) \\
{[\mathrm{kA}]} \\
\end{array}$ & $\begin{array}{c}\mathrm{J}_{o p} \\
{\left[\mathrm{~A} / \mathrm{mm}^{2}\right]} \\
\end{array}$ & $\begin{array}{l}\text { Ref. } \\
{[-]}\end{array}$ \\
\hline \multicolumn{6}{|l|}{ Single-Strand Conductors } \\
\hline Aegis Solenoid & NbTi & $1.25 \times 0.8$ & $0.2(5 \mathrm{~T}, 4.2 \mathrm{~K})$ & 194 & {$[31]$} \\
\hline ReBCO Coated Conductor** & $\mathrm{ReBCO}$ & $4 \times 0.096$ & $0.3(10 \mathrm{~T}, 4.2 \mathrm{~K})$ & 780 & \\
\hline \multicolumn{6}{|l|}{ Multi-Strand Cables } \\
\hline LHC Dipoles & $\mathrm{NbTi}$ & $15.1 \times 1.48$ & $11.85(8.3 \mathrm{~T}, 1.9 \mathrm{~K})$ & 530 & {$[32]$} \\
\hline FRESCA II & $\mathrm{Nb}_{3} \mathrm{Sn}$ & $21.3 \times 1.9$ & $10.9(13.4 \mathrm{~T}, 1.9 \mathrm{~K})$ & 270 & [33] \\
\hline Roebel* & $\operatorname{ReBCO}$ & $12 \times 1.1$ & $12.6(7 \mathrm{~T}, 4.5 \mathrm{~K})$ & 970 & [34] \\
\hline TSTC* & $\operatorname{ReBCO}$ & $\varnothing 7.4$ & $6.8(20 \mathrm{~T}, 4.5 \mathrm{~K})$ & 158 & {$[28]$} \\
\hline CORC Cable* & $\mathrm{ReBCO}$ & $\varnothing 7.6$ & $13(12 \mathrm{~T}, 4.5 \mathrm{~K})$ & 300 & {$[35]$} \\
\hline CORC Wire* & ReBCO & $\varnothing 3.6$ & $3(20 \mathrm{~T}, 4.5 \mathrm{~K})$ & 300 & [29] \\
\hline \multicolumn{6}{|l|}{ Cable-In-Conduit Conductors } \\
\hline CERN Superconducting Links & $\mathrm{MgB}_{2}$ & $\varnothing 65$ & 165 (1T, 4.5K) & 50 & [9] \\
\hline W7X Fusion Coils & $\mathrm{NbTi}$ & $16 \times 16$ & $16(6 \mathrm{~T}, 5 \mathrm{~K})$ & 63 & {$[36]$} \\
\hline ITER Toroidal Field Coils & $\mathrm{Nb}_{3} \mathrm{Sn}$ & $\varnothing 43.7$ & $68(12 \mathrm{~T}, 4.5 \mathrm{~K})$ & 45 & {$[8,37]$} \\
\hline ITER Central Solenoid & $\mathrm{Nb}_{3} \mathrm{Sn}$ & $49 \times 49$ & 45 (13T, 4.5K) & 19 & {$[8,37]$} \\
\hline ITER Poloidal Field Coils & $\mathrm{NbTi}$ & $52 \times 52$ & $52(5-6 \mathrm{~T}, 4.5 \mathrm{~K})$ & 19 & {$[8,37]$} \\
\hline Twisted Stacked Tape CICC** & $\operatorname{ReBCO}$ & $\varnothing 19$ & $20(15 \mathrm{~T}, 4.5 \mathrm{~K})$ & 55 & {$[38]$} \\
\hline Twisted Stacked Tape CICC* & $\operatorname{ReBCO}$ & $70 \times 19$ & $50(12 \mathrm{~T}, 8 \mathrm{~K})$ & 40 & [39] \\
\hline CORC CICC* & $\operatorname{ReBCO}$ & $30 \times 30$ & $80(12 \mathrm{~T}, 4.5 \mathrm{~K})$ & 89 & [35] \\
\hline
\end{tabular}

* Currently in development phase

** Standard ReBCO tape with $50 \mu \mathrm{m}$ of substrate and $20 \mu \mathrm{m}$ of copper stabilizer on both sides.

\subsection{Joints for ReBCO CORC Conductors}

Joints between ReBCO CORC conductors, or between any superconducting cables in general, are a key part co-determining the conductor's performance. Realizing a low joint resistance is important as it limits energy dissipation, while an equal contact resistance to each $\mathrm{ReBCO}$ tape ensures a homogeneous current distribution throughout the CORC conductor. Significant progress was already made towards practical CORC joint technology for single CORC cables in the years prior to the start of this work, resulting in compact cylindrical terminals in which the CORC cable is soldered [40]. However, in practice a low contact resistance remains problematic with this type of terminal as the Hastelloy substrate and the buffer layers in each ReBCO tape form a high-resistance barrier between layers in the CORC conductor. Similarly, homogeneous current distribution could not be achieved, since the Hastelloy substrate and the buffer layers are increasing the contact resistance to each subsequent layer. Improvements in the CORC joint technology is therefore highly desirable.

As part of this work, a novel type of joint terminal has been investigated that reduces the joint resistance, thereby also improving the current distribution in the CORC conductor. In parallel, a practical method for realizing this type of joints was developed and a nodalnetwork model of the joint terminals was designed, which is able to predict the joint resistance and to provide insight in the current distribution process in the CORC conductor. 
An important aspect of the new joint terminals is their scalability, from a terminal for a single CORC wire or cable all the way to a terminal for the more voluminous multi-strand CORC Cable-In-Conduit Conductor. Design and modeling results are elaborated in more detail in Chapter 3.

\subsection{Scope and Objective of the Thesis}

CERN has an interest in the CORC Cable-In-Conduit Conductor for possible use in future, high-current detector magnets and in the bus lines that feed current to these magnets; as well as in thin flexible CORC wire, this time for high-field accelerator magnets and insert coils. Therefore a joint research effort was established between CERN, Advanced Conductor Technologies and the University of Twente, aiming at further advancing the technology of CORC conductors.

The aim of this thesis work is to pioneer the development of ReBCO CORC conductors for application in large-scale magnet systems. This includes both the development of thin flexible CORC wires with high current density, as well as the design, preparation and testing of CORC multi-strand Cable-In-Conduit Conductors. As argued above, also the development of low-resistive joints for CORC wires, cables and Cable-In-Conduit Conductors, either interconnecting two CORC conductors or to connect a CORC conductor to a current lead, is an essential part of the work.

The research consists of the design and realization of demonstration CORC conductors, including the preparation of several CORC Cable-In-Conduit Conductors and their characterization, supported by modeling work on CORC conductors and their joint terminals.

The thesis is divided in the following chapters:

- In Chapter 1, a short introduction to HTS ReBCO-based conductors and their benefits for large scale magnets is presented.

- In Chapter 2, a more detailed description of the layout, performance, limits and the latest developments of ReBCO coated conductor, focusing on the design aspects required to prepare a CORC conductor from the ReBCO tapes is provided.

- In Chapter 3, current sharing between ReBCO tapes and layers within the CORC conductor is discussed and a novel design for joint terminals of the CORC conductors is introduced. This novel joint design aims to reduce contact resistance and to improve the current distribution between ReBCO tapes in the CORC conductor.

- In Chapter 4, two characterization experiments are described that were conducted to assess the performance of several state-of-the art CORC cables and wires. The experiments aimed specifically at identifying points of improvement that can be used to further optimize CORC performance. The tested CORC wires are for application in high-field magnets. Therefore, this chapter is concluded with a summary of the current status of several ReBCO based magnets and details on the design and preparation of a CORC-type racetrack coil at CERN. 
- In Chapter 5, the design, preparation and testing of three multi-strand CORC CableIn-Conduit Conductors is described. These conductors are designed for future implementation in magnet systems such as present in large particle detectors and fusion experiments, but also in the bus lines that feed relatively high currents to these magnets.

- In Chapter 6, the overall conclusions and recommendations for future development of CORC conductors are formulated.

To support reading the thesis, its outline is presented graphically in Figure 1.5.

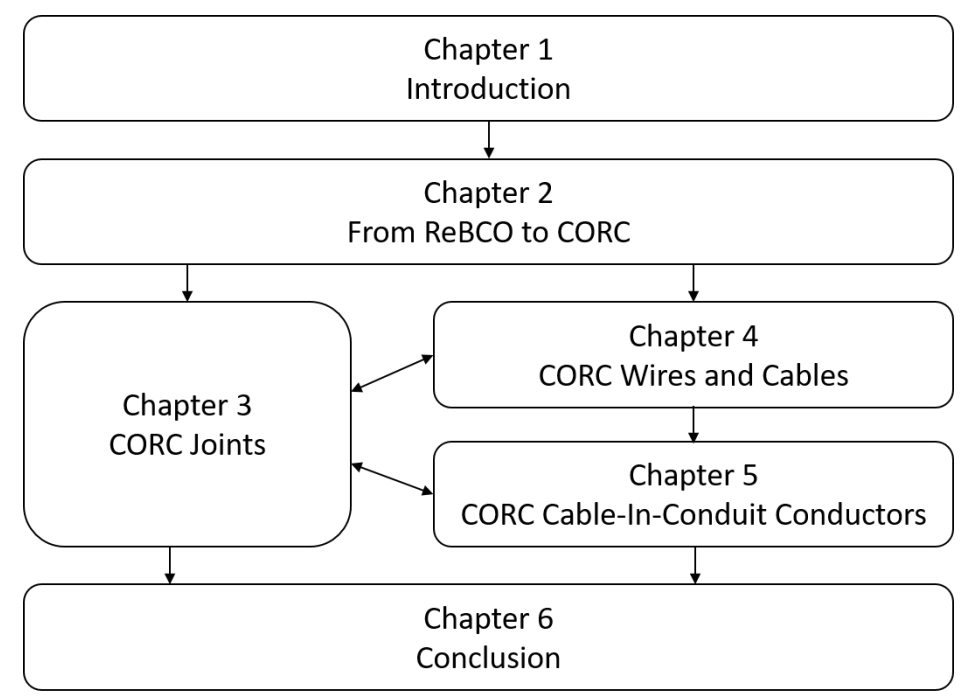

Figure 1.5: Chart with the outline of the thesis and the flow of information. 


\section{Chapter 2}

\section{From ReBCO Coated Conductor to Conductor On Round Core}

In this chapter, the development route from ReBCO coated conductor to the multi-tape CORC conductor is described. ReBCO tapes are the main ingredient of the CORC conductor and therefore key in its design. The tape production techniques, performance, mechanical behavior and limitations are addressed and recent advancements in the tape technology are highlighted. CORC conductors comprise many ReBCO tapes and benefit directly from advancements in tape technology. Aspects in the design and production of the CORC conductor, but also its mechanical behavior and limitations are reported.

\subsection{Introduction}

Conductor On Round Core (CORC) is a round flexible conductor that is based on HTS ReBCO coated conductor technology. The CORC conductor's round shape provides the advantage of flexible isotropic bending. This makes CORC appealing for superconducting power transport in confined spaces, such as navy vessels, but also for application in high-field superconducting magnets operated in the 2 to $4 \mathrm{~K}$ temperature range. In this temperature range the performance of ReBCO coated conductors out performs LTS niobium based alternatives by far and the round CORC shape allows practical magnet winding. Alternatively, CORC can be operated at elevated temperatures of 30 to $50 \mathrm{~K}$, a temperature range uniquely served by $R e B C O$. This offers a significant simplification of the cryogenic system and a reduction in cooling costs compared to operation at $4 \mathrm{~K}$.

CORC comes in many configurations, each tailored for its designated application. Thin ReBCO CORC wires with diameters smaller than $4 \mathrm{~mm}$ are made available due to recent advances in ReBCO tape technology. Throughout this thesis, we will use the $4 \mathrm{~mm}$ (arbitrary, but reflecting the present state-of-the-art) diameter as the boundary between relatively thin "wire" and thicker "cable" technology. CORC wires are much more flexible and also have a higher current density due to their thinner former. The CORC wire design 
is mainly focused on application in high-field magnets and insert coils operated at $4 \mathrm{~K}$ to generate magnetic fields of $20 \mathrm{~T}$ and above.

On the other side of the spectrum lay the multi-strand CORC Cable-In-Conduit Conductors (CICC) for large, high-current magnet applications such as magnets for particle detectors and experimental fusion facilities. These conductors are characterized by their relatively high thermal en electrical stability, in combination with high transport currents of over $80 \mathrm{kA}$ in magnetic fields exceeding $10 \mathrm{~T}$ at a temperature of $4 \mathrm{~K}$. CORC CICCs are discussed in detail in Chapter 5. A picture of a CORC wire and CORC cable is presented in Figure 2.1.

Several (multi-strand) CORC conductors in various configurations were designed, produced and tested in a joint effort between CERN, Advanced Conductor Technologies (ACT) company and the University of Twente. The general issues and challenges in the design and production of such conductors are discussed in this chapter. The primary component in each CORC conductor is the ReBCO coated tape conductor.

\subsection{ReBCO Coated Conductor Technology}

ReBCO coated conductors are superconducting ceramic tape conductors based on the crystal structure of a rare earth (Re) element combined with barium, copper and oxygen. The rare earth atom is commonly Yttrium forming an $\mathrm{YBa}_{2} \mathrm{Cu}_{3} \mathrm{O}_{x}$ (YBCO) crystal structure, but can be interchanged with several chemically similar rare earth elements, e.g. gadolinium (GdBCO), each of which has advantages and disadvantages. The superconducting layer has to be grown with high precision to align its crystal structure. If not, the grain boundaries between multiple unaligned domains, with a misorientation of over only 2 to 3 degrees, form resistive barriers that greatly reduce the flow of current [41]. Relatively simple extrusion or Powder in Tube (PiT) production methods are therefore unavailable for this material, so that more advanced and costly methods are used. There are multiple routes in the production of coated conductors, all comprising a pre-texturing and a deposition step under vacuum conditions. The pre-texturing of the substrate step

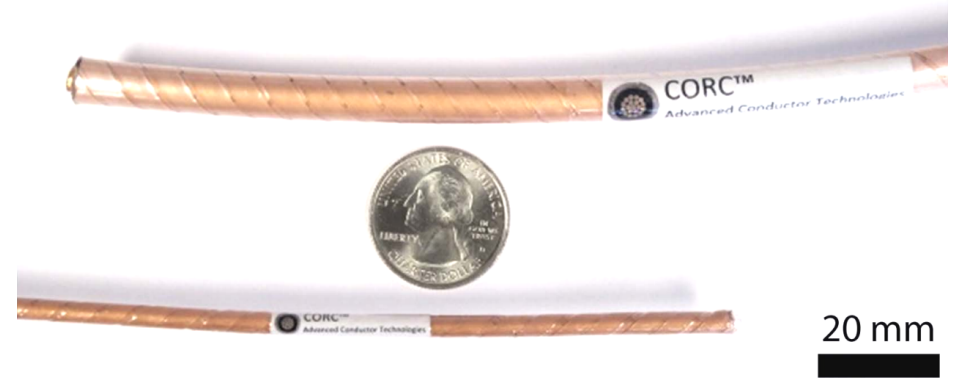

Figure 2.1: Picture of a thin CORC wire $(\varnothing=3 \mathrm{~mm})$ aimed for use in high-field magnets and inserts, compared to a thicker CORC cable $(\varnothing=8 \mathrm{~mm})$ that can for example be used as a strand in a CORC CICC [29]. 
ensures the alignment during the deposition and growth of the buffer and ReBCO layers. Two common texturing techniques are:

- Ion Beam Assisted Deposition (IBAD), where the substrate is ionized during the deposition step, which catalyzes the growth of a textured buffer layer [42].

- Rolling Assisted Biaxially Textured Substrate Technology (RABiTS), where the substrate is annealed and its microstructure is mechanically textured via a rolling process [43].

Both texturing methods produce ReBCO tape with the ReBCO layer attached to either a steel or a Hastelloy C-276 substrate. The substrate thus serves as a relatively strong mechanical support for the 1 to $2 \mu \mathrm{m}$ thick layer of ReBCO. Several buffer layers are applied to smoothen the transition between the lattice structures of the substrate and the ReBCO layer and to avoid chemical diffusion between both, which would corrupt the ReBCO coating. Deposition of the buffer and ReBCO layers can be achieved via several methods:

- Metalorganic Deposition (MOD): here the tape's surface is coated with a thin liquid organic film. The film then undergoes a chemical reaction with added gas, which forms the buffer and ReBCO layers [44];

- Metalorganic Chemical Vapor Deposition (MOCVD): similar to MOD, but with high purity vapors instead of the liquid film [45];

- Pulsed Laser Deposition (PLD): here a high-power laser pulse vaporizes target material that is then deposited as a thin film on the substrate [46];

- Reactive Co-Evaporation (RCE): different elements are evaporated in vacuum and deposited individually on the substrate [47].

An overview of the production process routes used by selected ReBCO coated conductor manufacturers is presented in Table 2.1. For chemical protection, the ReBCO layer is capped with a thin coating of silver. Often, a copper stabilizer is additionally electroplated on one side or both sides of the tape or fully surround the tape to offer a parallel path for current (and heat) and thus to increase the tape's thermal stability, albeit at the cost of current density. A schematic layout of a characteristic ReBCO coated conductor is presented in Figure 2.2.

At the time of writing, over 10 different ReBCO tape manufacturers are active. This relatively large number reflects the world-wide interest in the potentially wide applicability of ReBCO tape technology. Especially in the last couple of years new developments in tape technology succeeded each other rapidly; better process control has improved the reproducible performance of the tapes drastically (boosting both the critical current density $\mathrm{J}_{e}$ and production yield), the thickness of the substrate is significantly reduced, additional Zr doping allows for improved in-field performance, etc.. Especially the thinner substrates in the tape from SuperPower have proven to be instrumental in improving CORC technology. The substrate thickness not only dictates the minimum bending diameter of the ReBCO tapes, which directly effects the minimum diameter of the CORC conductor, but 
Table 2.1: Some selected ReBCO coated conductor manufacturers and there production routes[48].

\begin{tabular}{ll}
\hline \hline Manufacturer & Route \\
\hline American Superconductor (AMSC) & RABiTS/MOD \\
Bruker & IBAD/PLD \\
Fujikura & IBAD/PLD \\
SuNAM & IBAD/RCE \\
Sumitomo & RABiTS/PLD \\
SuperOx & IBAD/PLD \\
SuperPower & IBAD/MOCVD \\
STI & IBAD/RCE \\
\hline \hline
\end{tabular}

also allows to increase the tape's overall current density, since the total tape thickness is reduced while its critical current remains the same.

\subsubsection{Tape Performance and Recent Advancements}

The performance and configuration of ReBCO tapes vary considerably between tape manufacturers. The tape configurations differ in width, stabilizer, substrate thickness and the location of the stabilizer. Two examples of very different ReBCO tapes are presented in Figure 2.3. The $45 \mu \mathrm{m}$ thin and $3 \mathrm{~mm}$ wide tapes are from SuperPower Inc., used e.g. in thin CORC wires, while the $12 \mathrm{~mm}$ wide and much thicker, $\sim 205 \mu \mathrm{m}$, tape is from THEVA, designed for implementation in the large coils of the first superconducting wind turbine in the ECOSWING project [50]. The CORC conductor mainly uses tapes from SuperPower for their thin substrate, therefore the focus here is on tapes from this manufacturer. Tapes from SuperPower are named by their type SCS (Surround Copper Stabilizer) or SF (Stabilizer Free) followed by their width in $\mathrm{mm}$ and their substrate thickness in $\mu \mathrm{m}$. SCS4050

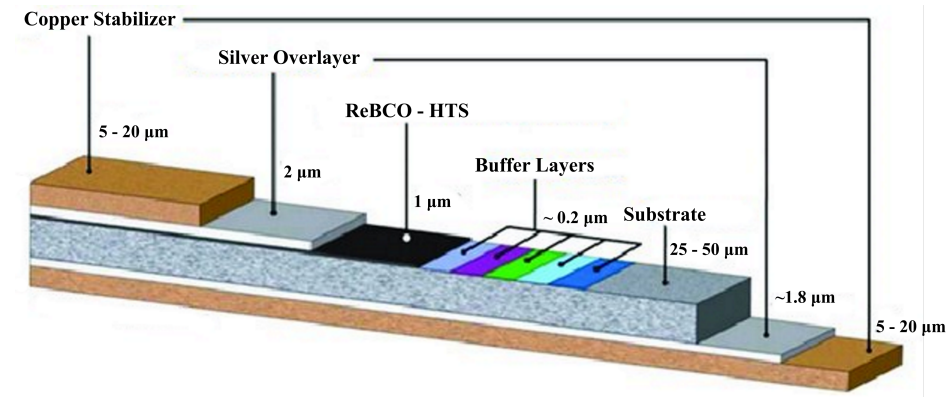

Figure 2.2: Layout of a ReBCO based coated conductor of the company SuperPower Inc. [49]. The substrate serves as mechanical support, the buffer layers offer a chemical barrier and a transition between the lattice structure of the substrate and the ReBCO layer. The silver and copper layers improve the tape's electrical and thermal stability and also allow to make direct and simple electrical connections to the tape's surface. 
is the most commonly used tape from SuperPower for CORC cables with thus a width of $4 \mathrm{~mm}$ and $50 \mu \mathrm{m}$ of Hastelloy substrate and the SCS2030 type of tape is mainly used for CORC wires for its narrow $2 \mathrm{~mm}$ width and thin $30 \mu \mathrm{m}$ of substrate.

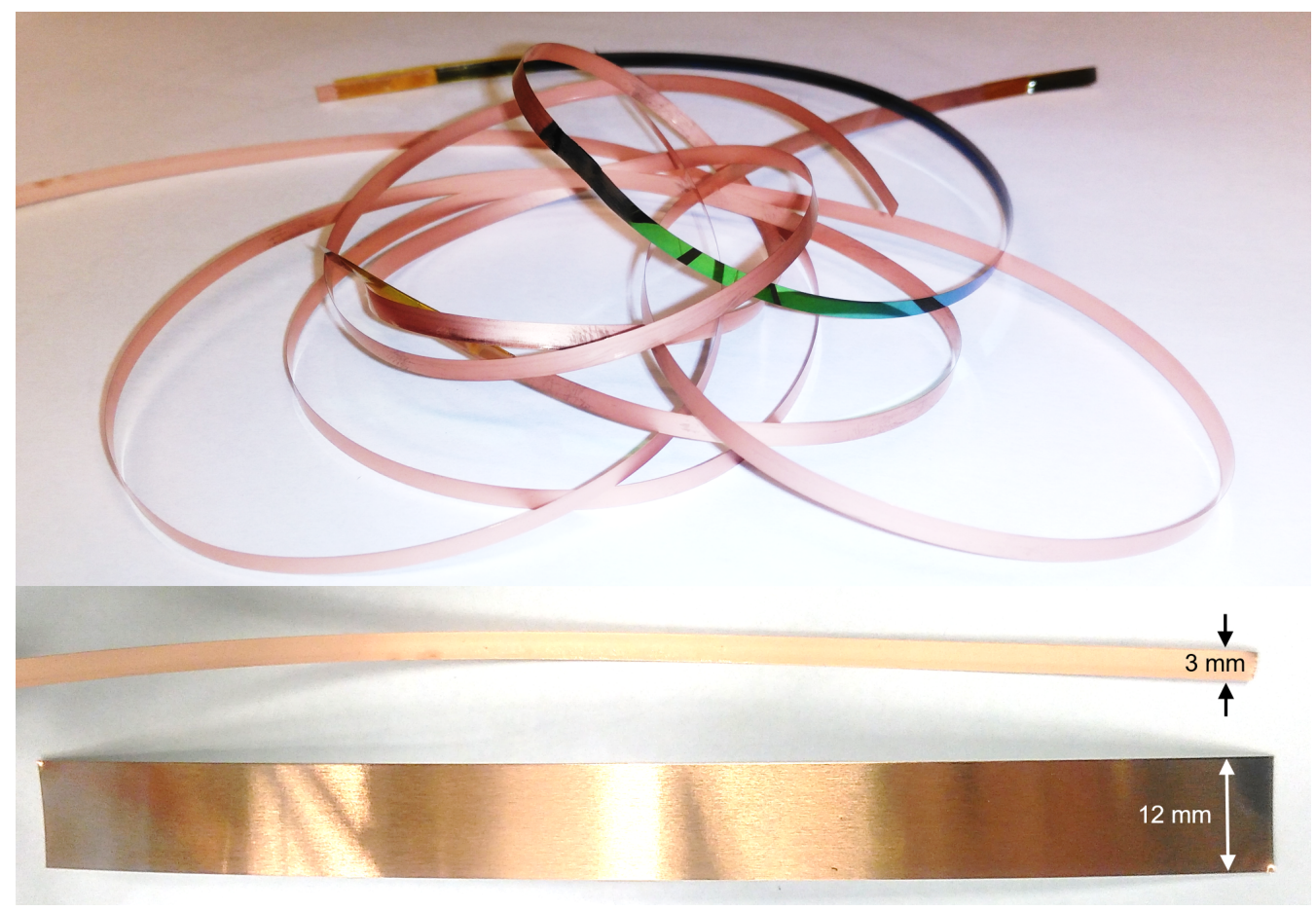

Figure 2.3: Top picture shows a ReBCO tape of $3 \mathrm{~mm}$ wide that has a $30 \mu \mathrm{m}$ thick Hastelloy substrate and $5 \mu \mathrm{m}$ of copper stabilizer plated on each side of the conductor. The ends of the tape are not yet plated and the ReBCO and buffer layers are visible. This tape is relatively flexible and has a high engineering current density due to its thin substrate and stabilizer. Bottom picture shows the same $3 \mathrm{~mm}$ wide tape next to a $12 \mathrm{~mm}$ wide tape with a $100 \mu \mathrm{m}$ thick substrate and $100 \mu \mathrm{m}$ of copper stabilizer, which is mechanically more robust and thermally more stable than the thin flexible tape at the cost of a much lower engineering current density.

The performance of ReBCO tapes, or any other superconducting wire, can be described in terms of critical current and n-value. Both values depend on magnetic field, magnetic field-angle, temperature and mechanical strain. Together, these two quantities describe the current-voltage characteristics of the tapes, often described as a power-law:

$$
E=E_{0}\left(\frac{I}{I_{c}(B, \phi, T, \epsilon)}\right)^{n(B, \phi, T, \epsilon)} .
$$

Here $E$ is the electric field along conductor, $E_{0}$ is an electric field criterion (usually 10 or $100 \mu \mathrm{V} / \mathrm{m}), I$ is the operating current, $I_{c}$ is the critical current of the tape and $n$ is the tape's n-value. The $\mathrm{n}$-value is rather similar between different tape manufacturers, 
with a value around 30 at $77 \mathrm{~K}$ and 40 to 60 at $4.2 \mathrm{~K}$ [51]. The critical current as function of field and temperature varies substantially between different producers. $\mathrm{I}_{c}$ measurements for many commercially available tapes can be found in literature [51, $52,53]$. A recent example of such critical current measurement on commercial tapes is presented in Figure 2.4, which illustrates the large diversity in the performance of the tapes. It also shows the advancement in tape performance for one manufacturer by doping-induced flux pinning.

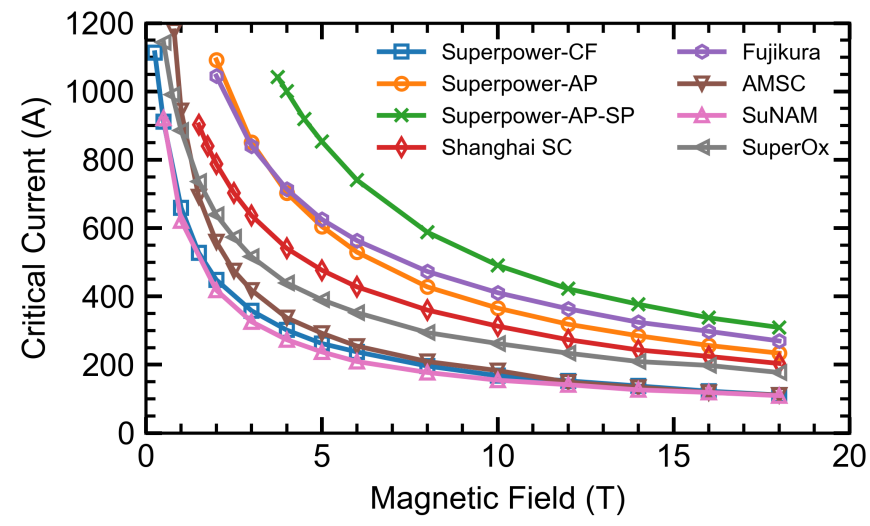

Figure 2.4: Critical current as function of magnetic field for $4 \mathrm{~mm}$ wide tapes from several ReBCO CC manufacturers, reported by Tsuchiya et al. [51]. The tapes with increased doping, especially the Superpower-AP-SP ( $A P=$ Advanced Pinning, $S P=$ Special, due to its thin $30 \mu \mathrm{m}$ substrate), perform significantly better in magnetic field compared to tapes without doping such as the Superpower-CF tape.

The magnetic field dependence of the critical current in ReBCO tapes is anisotropic, i.e. $\mathrm{I}_{c}$ does not only depend on the magnitude of the magnetic field, but also on its orientation with respect to the conductor. The tapes perform best in a magnetic field parallel to the tape's surface in the crystallographic $a b$-plane and worst with the magnetic field perpendicular to the tape's surface along the crystallographic c-axis. A perpendicular magnetic field causes a larger reduction in the tape's critical current, as can be seen in Figure 2.5. Roebel and Twisted Stacked ReBCO conductors have the advantage that they can in principle be mounted such that all tapes everywhere experience a parallel magnetic field. This is not the case with the CORC conductor, where all tapes experience both parallel and perpendicular magnetic field components along one twist pitch. Depending on the tape-to-tape contact resistance, current sharing between tapes may improve $\mathrm{I}_{c}$ performance as the current in tapes that locally experience a perpendicular field can redistribute to tapes that do not.

Improved tape performance in a magnetic field can be achieved by adding artificial impurities (doping) to the ReBCO layer. These impurities serve as pinning centers for the magnetic flux-lines. Presently, the impurity of choice is often Zirconium ( $\mathrm{Zr}$ ), as this element induces the growth of self-assembled $\mathrm{BaZrO}_{3}$ (BZO) nanorods along the crystallographic c-axis that greatly improve the tape's critical current density in magnetic 


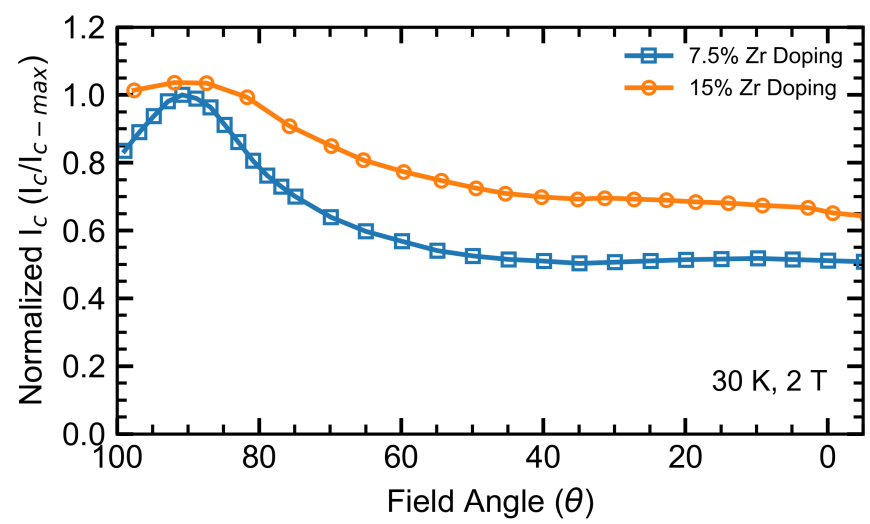

Figure 2.5: Critical current as function of field angle for different amounts of Zr doping [56].

field perpendicular to its surface (B//c) [54, 55]. Currently, ReBCO tapes with $7.5 \%$ of $\mathrm{Zr}$ doping are commercially available. A further increase of $\mathrm{Zr}$ doping from $7.5 \%$ to $15 \%$ is currently under development and can provide a further boost in critical current density in the mid- to high-magnetic field range, but it causes a reduction of the tape's performance in low-magnetic fields [56].

An analytical expression was developed by Hilton et al. that describes the transport current of ReBCO tape as function of magnetic field and field angle with respect to the tape's surface at constant temperature [57]. This expression was fitted to measured data of several commercially available ReBCO CCs by Hilton et al.. The fitting equation is given by:

$$
I_{c}(B, \theta)=\frac{k_{0}}{\left(B+\beta_{0}\right)^{\alpha_{0}}}+\frac{k_{1}}{\left(B+\beta_{1}\right)^{\alpha_{1}}} \cdot\left[w^{2}(B) \cos ^{2}\left(\theta-\phi_{1}\right)+\sin ^{2}\left(\theta-\phi_{1}\right)\right]^{-1 / 2},
$$

where

$$
w(B)=c\left[B+\left(\frac{1}{c}\right)^{\frac{5}{3}}\right]^{\frac{3}{5}} .
$$

Here the $I_{c}, B$ and $\theta$ are the tape's critical current, magnetic field and field angle. The fitting parameters are $k_{i}, \alpha_{i}, \beta_{i}$ and $c$, while a possible offset in the position of the tape's peak critical current is incorporated by $\phi_{i}$. The fitting parameters for SuperPower SCS4050-AP tape are provided in Table 2.2. ReBCO tapes in the CORC conductor experience the full range of field angles within one twist pitch. Their critical current thus changes as function of their longitudinal position along the CORC conductor and rotation with respect to the magnetic field, which has to be accounted for while estimating the CORC conductor's performance, which is the primary use of Equation 2.2 throughout this work. 
Table 2.2: Parameters obtained by fitting Equations 2.2 and 2.3 to critical current data for SuperPower SCS4050-AP tape at $4.2 \mathrm{~K}$ derived by Hilton et al. [57].

\begin{tabular}{ll}
\hline \hline Parameter & Value \\
\hline$k_{0}$ & 8870 \\
$k_{1}$ & 18500 \\
$\alpha_{0}$ & 1.30 \\
$\alpha_{1}$ & 0.809 \\
$\beta_{0}$ & 13.8 \\
$\beta_{1}$ & 13.8 \\
$\phi_{1}$ & -0.180 \\
$c$ & 2.15 \\
\hline \hline
\end{tabular}

The critical current density of the tapes can be further increased by reducing the thickness of both substrate and stabilizer. In 2017, SuperPower started the commercial production of ReBCO coated conductors with a substrate thickness of $30 \mu \mathrm{m}$, in parallel with their standard tape with $50 \mu \mathrm{m}$ thick substrate, to serve the large demand of thinner, high $\mathrm{J}_{c}$ tapes [58]. A further reduction of the thickness from 30 to 25 (and maybe 20) $\mu \mathrm{m}$ is being investigated and may see production in the years to come. This development yields about a doubling of the engineering current density of the tapes that is very beneficial for high-field application in coils beyond $20 \mathrm{~T}$.

Another development in the tape technology is the reduction of the tape width, either by the production of narrower tape or by striation of wider tapes (laser engraving multiple narrower tapes on one wide tape). Narrow tapes show reduced AC loss [59] and improved flexibility enabling smaller diameter CORC wires. The standard tape widths of SuperPower tapes are 4 and $12 \mathrm{~mm}$. Recently, tapes of 3 and $2 \mathrm{~mm}$ wide with thinner substrate of $30 \mu \mathrm{m}$ went in production and became commercially available.

\subsubsection{Strain and Tape Bending}

The 1 to $2 \mu \mathrm{m}$ thick ReBCO layer itself is a brittle ceramic. Stress inside the ReBCO layer can introduce resistive microscopic cracks in the superconductor [60], which may lead to permanent degradation of the tape's superconducting properties. The amount of strain that causes degradation in the overall tape performance depends on the deposition process the manufacturer is using and on the tape configuration; especially the thickness and position of substrate and stabilizer material. More $\mathrm{Cu}$ stabilizer causes an increase of the irriversible strain limit of the tapes $[61,62]$. The strain dependence of the critical current of ReBCO CCs has been measured and is described abundantly in literature [63, 64, 65]. Measurements show that ReBCO tapes are more vigilant in the in-plane compressive strain regime, which allows smaller tape bending radii than in the tensile strain regime. Typical strain limits are $+0.5 \%$ in the tensile strain regime and -0.8 to $-1.0 \%$ in the compressive strain regime, as demonstrated by Otten et al. [65]. A sharp decrease in critical current is observed upon reaching the tensile strain limit, after which irreversible and severe degradation in tape performance occurs. Compressed ReBCO layers show a 


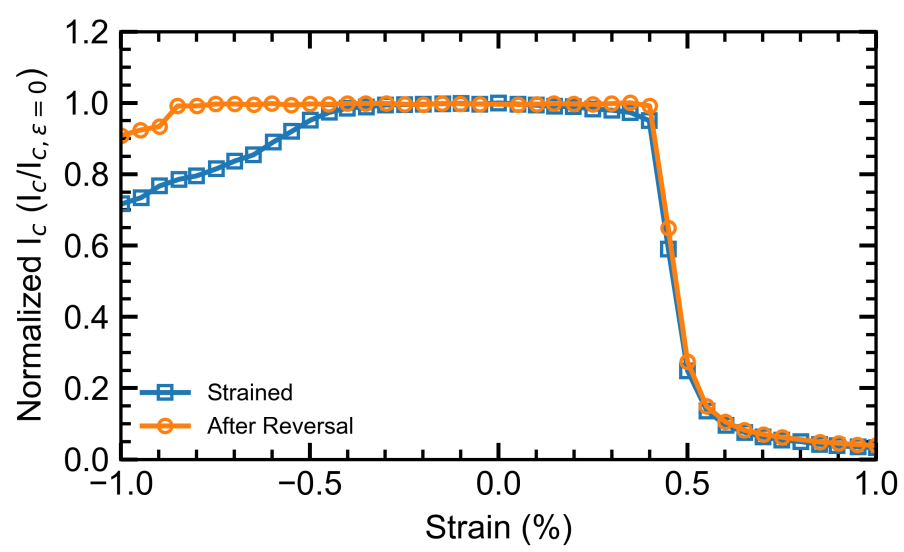

Figure 2.6: Effect of tensile and compressive in-plain strains on the critical current of SuperPower SF12100 (SF = Stabilizer Free) tape reported by Otten et al. [65]. The graph shows that ReBCO CCs have a higher limit at compressive strain compared to tensile strain. In the compressive strain regime the critical current of the tape fully recovers up to $-0.8 \%$ strain, while the tape permanently degrades at a tensile strain beyond $0.5 \%$.

slower decline in performance as function of strain after reaching a compressive strain limit, which is in many cases reversible after the strain is released, as shown in Figure 2.6. Tapes in CORC conductors are therefore wound with the ReBCO layer facing inwards to make optimal use of their strain dependent properties.

Similarly, ReBCO coated conductors have a high tolerance to transverse compressive strain, where degradation of a bare unstabilized coated conductor is observed only at stress beyond $300 \mathrm{MPa}$ [66]. Transverse tensile strain causes ceramic layers delaminate from each other at low stress below $15 \mathrm{MPa}$ [67] and results in severe degradation of the tape's critical current. However, tensile transverse stresses on the tapes are absent in normal operation of a CORC conductor as it mainly experiences transverse compressive loads due to the electro-magnetic Lorentz forces.

The amount of in-plane strain in the ReBCO layer depends on the bending diameter of the tape and on the distance between the tapes strain-free neutral axis and the ReBCO layer. Most ReBCO tapes have their Hastelloy substrate in the center of the tape, with the ReBCO+buffer layers on one side of the substrate and copper stabilizer material plated on both sides. Bending the tapes strains the ReBCO layer positively (tensile) or negatively (compressive) depending on the bending direction, as presented in Figure 2.7. The strain $\epsilon$, either compressive or tensile, in the ReBCO layer as function of substrate thickness and bending radius is given by:

$$
\epsilon(r, t)=\frac{l_{\text {ReBCO }}-l_{\text {neutral }}}{l_{\text {neutral }}}=\frac{2 \pi(r \pm t / 2)-2 \pi r}{2 \pi r}=\frac{ \pm t}{2 r}
$$




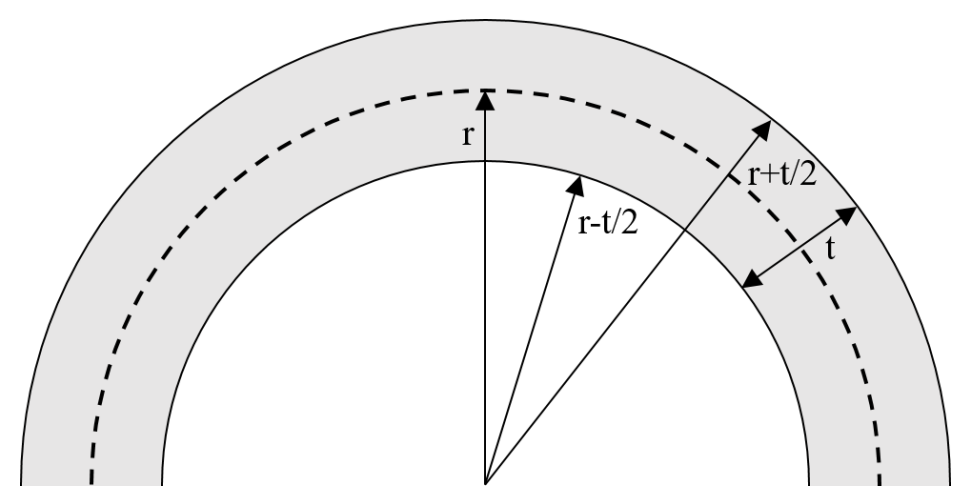

Figure 2.7: Schematic view of tape bending, with the neutral plane in the center of the Hastelloy substrate indicated by the dashed line. The ReBCO layer is located at either $r-t / 2$ or at $r+t / 2$, which has influence on the strain in the ReBCO layer.

Here $t$ is the thickness of the substrate and $r$ the bending radius of the tape with respect to the center of the Hastelloy substrate, which is assumed to be the strain free neutral plane, as shown in Figure 2.7. For example, the ReBCO layer of a tape with $50 \mu \mathrm{m}$ of Hastelloy substrate is strained $1.2 \%$ for a bending radius of $2.1 \mathrm{~mm}$.

Tapes in CORC conductors are wound in a helical fashion, which requires a small alteration in equation 2.4 to include the twist pitch $p$ of the tape:

$$
\epsilon(r, t, p)=\frac{\sqrt{p^{2}+4 \pi^{2}\left(r \pm \frac{1}{2} t\right)^{2}}}{\sqrt{p^{2}+4 \pi^{2} r^{2}}}-1 .
$$

The introduction of the pitch length $p$ causes a reduction of the strain in the tape's longitudinal direction. The tape's strain in the former's azimuthal direction still obeys Equation 2.4. Any resulting micro-cracks in the ReBCO layer are therefore less likely to extends over the entire width of the tape and will not cause a large reduction in performance.

A thinner substrate allows to reduce the minimum bending radius of the tapes and thus directly reduce the minimum diameter of the CORC conductor. A thinner core can be used, which significantly increases the overall current density of the conductor. The minimum bending radius of $\operatorname{ReBCO}$ tapes with $30 \mu \mathrm{m}$ of Hastelloy substrate is around $1.2 \mathrm{~mm}$ for $1.2 \%$ of compressive strain, which allows the production of CORC wires with diameters of 2.5 to $4 \mathrm{~mm}$. A further decrease in substrate thickness from 30 to $25 \mu \mathrm{m}$ will reduce the minimum CORC diameter to $1.8 \mathrm{~mm}$, which further boosts the conductors current density and thus its potential for implementation in high-field magnets. The current densities that can be reached with tapes of various substrate thicknesses are presented in Figure 2.8. The figure clearly shows that not only the diameter of the CORC conductor is reduced drastically by going to thinner substrate, but also the current density is boosted, which makes thinner substrates a very important ingredient in the ongoing development of thin CORC conductors. 


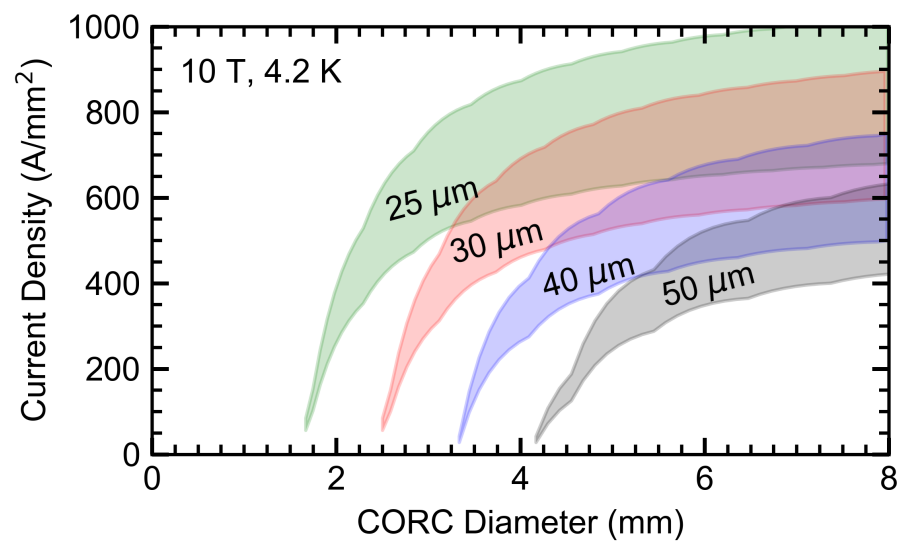

Figure 2.8: Predicted overall current density as function of conductor diameter for CORC conductors using ReBCO CCs with substrate thicknesses ranging from 25 to $50 \mu \mathrm{m}$. A lift factor of 2 to 3 is used to scale the current from $77 \mathrm{~K}$ and self-field to $4.2 \mathrm{~K}$ and $10 \mathrm{~T}$. Thinner substrate allows for thinner CORC conductors with diameters as small as $1.8 \mathrm{~mm}$. CORC wires manufactured with $25 \mu \mathrm{m}$ substrate bring current densities of $800 \mathrm{~A} / \mathrm{mm}^{2}$ at $10 \mathrm{~T}$ and $4.2 \mathrm{~K}$ within reach (corresponding to $400 \mathrm{~A} / \mathrm{mm}^{2}$ at $20 \mathrm{~T}$ and $4.2 \mathrm{~K}$ ), which makes them very suitable for high field magnets and inserts.

\subsection{CORC Conductor Geometry, Bending and Challenges}

\subsubsection{Geometry of CORC}

ReBCO coated conductors are helically wound in several layers mostly on a round metallic core to form the CORC conductor, as presented in Figure 2.9. Tubes or some metallic cable structure can be considered as core as well. Each layer comprises two to four $\mathrm{Re} \mathrm{BCO}$ tapes wound in parallel depending on the winding radius. Layers are laid down alternately in clockwise and counter-clockwise direction for improved conductor handling and reduction of the solenoidal component in the conductor's self field. Spiraling of the tapes introduces a twist pitch, which reduces AC loss. The tapes in the CORC conductor are not fully transposed (in which case each tape would experience an identical environment along one twist pitch, such as in Rutherford or Roebel cables), but they remain within their layer. Each additional layer of tapes increases the current density of the conductor, as the volume fraction of the conductors core is reduced.

The tapes are wound with winding angles $\alpha$ between 30 and 60 degree with some little spacing of 0.3 to $0.7 \mathrm{~mm}$ in between, as illustrated in Figure 2.10. The winding angle depends on the type of CORC conductor. CORC wires with narrow tapes tend to have smaller winding angles and thus shorter twist pitches to maintain the conductors flexibility. Thicker CORC cables with standard $4 \mathrm{~mm}$ wide tape have larger angles and longer twist pitches. The spacing has to be optimized for bending of the CORC conductor, which is explained in more detail in Section 2.3.2. The amount of tape per conductor varies as the twist pitch and winding radius can be different in each conductor configuration. The 
length of tape required to prepare a CORC conductor is given by the sum of the lengths of the individual tapes:

$$
l=L \sum_{i=1}^{n} \frac{\sqrt{\left(2 \pi r_{i}\right)^{2}+p_{i}^{2}}}{p_{i}}
$$

where $l$ is the total required length of tape material, $L$ is the conductor length, $r_{i}$ is winding radius of a given tape and $p_{i}$ its twist pitch. The first large CORC order of CERN in 2014 featured a CORC cable of $12.2 \mathrm{~m}$ in length that comprised 38 SuperPower SCS4050 ReBCO tapes wound in twelve layers. The conductor had a record length at that time and incorporated $610 \mathrm{~m}$ of ReBCO tape; which averaged to $1.31 \mathrm{~m}$ of tape per meter of conductor per tape. A $1.78 \mathrm{~m}$ long CORC wire was prepared for a measurement at the University of Twente with 29 ReBCO SCS2030 tapes. The CORC wire comprised a total of $80 \mathrm{~m}$ of ReBCO tape, which averaged to $1.55 \mathrm{~m}$ of tape per meter of conductor per tape. Typically, the tape efficiency (conductor length / tape length per tape) of the CORC conductor is in between 0.6 and 0.9, depending on the type of CORC conductor. For comparison, the Roebel conductor has a lower tape efficiency compared to the CORC conductor, since during preparation of tapes for the Roebel conductor just over half of the tape's volume is 'punched' away to create the required meandering tape layout [68]. The resulting tape efficiency is around 0.5. The Twisted Stacked conductor obviously has maximum tape efficiency compared to both CORC and Roebel conductor, since the tapes run parallel, are stacked and straight and thus it has an efficiency of 1.0.

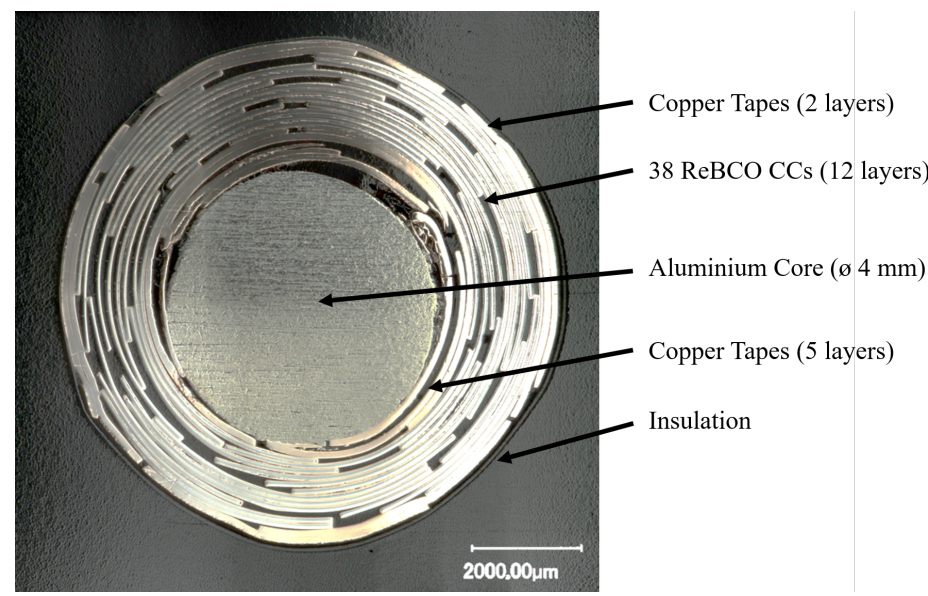

Figure 2.9: Picture of the cross-section of a CORC cable that comprises an aluminium round core, 38 ReBCO tapes wound in 12 layers and some additional copper tapes around the core and on the outside for mechanical protection.

The core of the CORC conductor can be a metal rod or tube that defines the bending radius of the ReBCO tapes. The material of the core is commonly Oxygen Free High Conductivity (OFHC) copper or electrical aluminum, since these material act as stabilizer material for improved electrical and thermal stability. Another possibility is to take a tube as conductor 


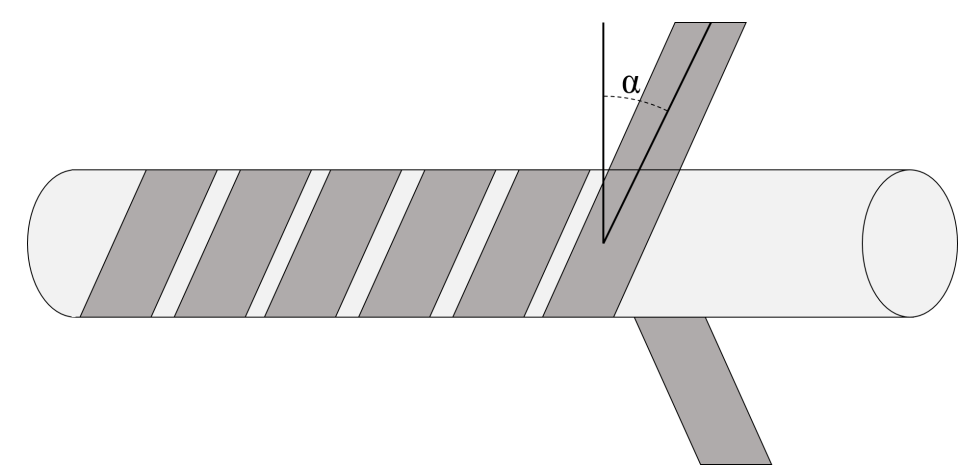

Figure 2.10: Schematic illustrating the winding angle of the ReBCO tapes.

core, which allows internal forced-flow cooling of the CORC conductor. The feasibility of a tube as core depends on the tube's bore diameter and conductor length, as the pressure drop over its length might become too high for effective forced-flow cooling.

\subsubsection{CORC Bending}

One of the main advantages of the CORC conductor is it's omni-directional bending tolerance. The CORC conductor can therefore be bent to various shapes and forms, as shown e.g. in Figure 2.11. The mechanism behind the flexibility of CORC conductors is that the tapes in different layers are able to slide over each other to a position where they experience the least amount of strain. Nevertheless, bending a conductor that is based on flat ceramic tapes is not trivial and requires a delicate touch. Permanent degradation may occur when a CORC conductor is bent over a radius that is too small, as demonstrated by D. van der Laan et al. and J. Weiss et al. in [29] and [69].

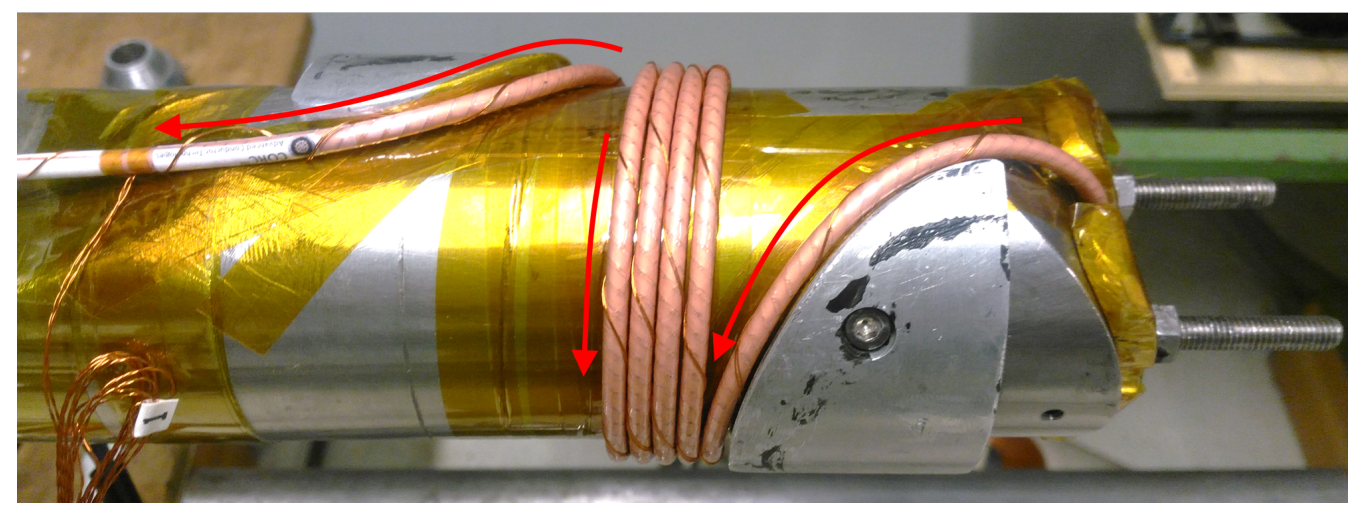

Figure 2.11: CORC wire of $3.5 \mathrm{~mm}$ in diameter bend on the Twente Sample holder, involving 3 different bending directions. 


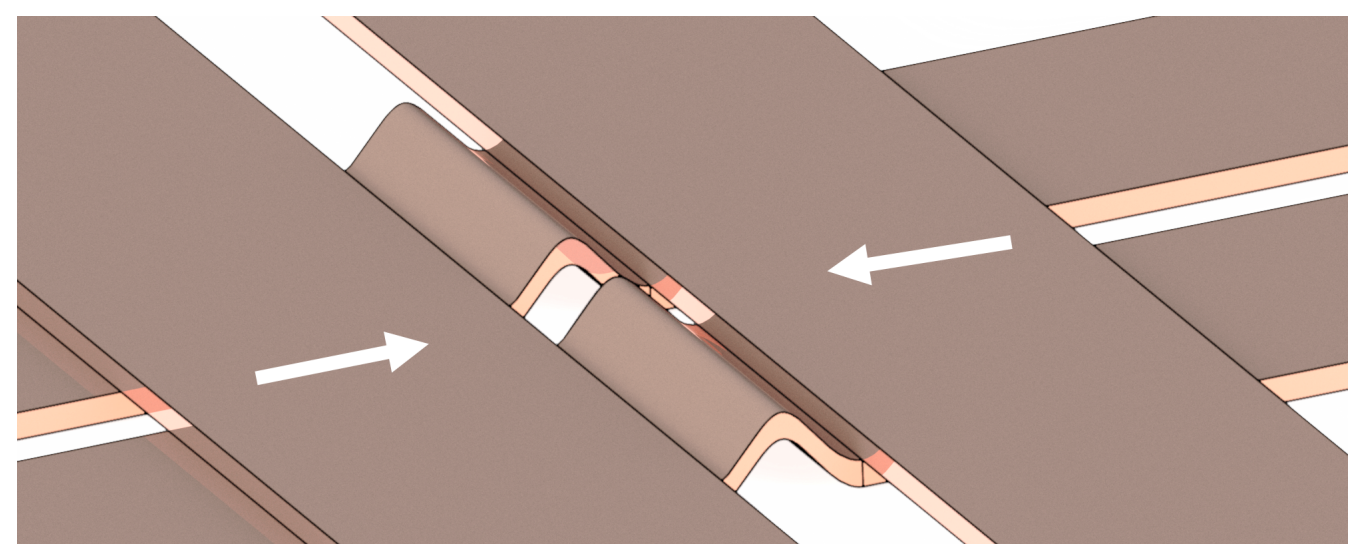

Figure 2.12: 3D representation of the tape buckling process to be avoided during CORC production and application. The upper tapes slide while bending the CORC cable. When there is too much friction between layers, the tapes in the bottom layer get caught between tapes of in the upper one, causing them to buckle.

One of the issues that may arise when bending a CORC conductor is tape buckling (tapes give way under compressive pressure or strain). Buckling usually occurs due to the combination of loosely wound tapes and bending of the CORC conductor or a gap spacing between tapes that is too large. While sliding, a loosely wound tape can be caught between two other tapes as is illustrated in Figure 2.12. Buckling will likely lead to a large or total reduction of the conductor's $\mathrm{I}_{c}$ around the region with the buckled tapes. Buckling can be avoided by increasing the winding tension, which makes it less likely for a tape to be caught between other tapes while bending [70] and by limiting the gap spacing. Another option is to add some lubrication to the tapes, which reduces the sliding friction and therefore makes it less likely a tape will get stuck between other tapes. CORC cabling thus requires optimization of inter-tape spacing, lubrication, tape tension and constant control of parameters to arrive at a uniform product.

Often 2 or 3 tapes are wound in parallel in a single layer. The distance between the tapes is another important aspect in the design of the CORC conductor. It is impossible to bend a CORC conductor that has no space between tapes, but it is also undesirable to have a too large distance between tapes as it reduces the current density of the CORC conductor. The distance between tapes can be optimized for the desired bending radius of the CORC conductor. When a CORC conductor is bent, the tapes on the inner radius of the conductor need to slide a given distance towards each other, which reduces the spacing between them. Otherwise adjacent tapes are crammed together, which can cause degradation of their performance. The minimal distance between tapes can be calculated with the following equations:

$$
\frac{c_{\text {inner }}}{c_{\text {neutral }}}>\frac{w}{w+s}
$$




$$
\frac{2 \pi(r-d)}{2 \pi r}>\frac{w_{0} / \cos (\alpha)}{w_{0} / \cos (\alpha)+s} .
$$

Here $c$ is the circumference of the neutral or inner radius of the CORC conductor, $r$ is the radius of the neutral axis, $d$ is the distance of the tapes with respect to the conductor's neutral axis, $w$ is the effective tape width in the conductors longitudinal direction, $w_{0}$ is the original tape width (commonly either 4,3 or $2 \mathrm{~mm}$ ), $\alpha$ is the tapes winding angle and $s$ is the distance between tapes. There is still some distance between tapes after bending when the ratio between inner and neutral radius of the CORC conductor is larger than the ratio between tape width and tape width + spacing. Therefore the minimum spacing $s_{\min }$ between tapes for a given bending radius and CORC conductor diameter is given by:

$$
s_{\text {min }}=\frac{d}{r-d} w_{0} / \cos (\alpha) .
$$

For example, the minimum distance between tapes in the outer layer of a CORC wire with a diameter of $3.5 \mathrm{~mm}, 2 \mathrm{~mm}$ wide tapes wound at an angle of 45 degrees that will be bent around a former with outer radius of $30 \mathrm{~mm}$ is $0.16 \mathrm{~mm}$.

\subsubsection{Production Process}

The CORC conductor is manufactured by the start-up company Advanced Conductor Technologies in Boulder, CO, USA. Conductor winding starts with the conductor's core. The core is pulled through the cabling machine while one layer of tapes (usually multiple tapes in parallel) is wound around it. This process is repeated for each additional layer. In this 'reel-to-reel' process, the length of the CORC conductor is in principle limited only by the lengths of the tapes and the core, and the spool-size of the winding machine. A picture of the cabling machine is presented in Figure 2.13. Usually, a polyethylene insulation sleeve is fitted around the CORC conductor after production, but other options exist. The sleeve holds all tapes together and prevents some of the buckling as it limits the outwards movements of the ReBCO tapes during conductor bending.

A challenge in the design and production is the application of the right amount and uniformity of tensile winding load to the ReBCO tapes during the production process. Not enough pre-load on the tapes results in loosely wound tapes, which can lead to buckling as explained in the previous section. Excessive pre-load can cause some degradation as the resulting stress in the ReBCO layer can produce microscopic cracks. The thickness of the Hastelloy substrate and the amount of stabilizer has an influence on the amount of pre-load that a single tape can handle. It is still under investigation what the best load settings are for the different types of ReBCO tapes and adjustments are required case by case.

Each test performed on a CORC conductor provides valuable feedback for further optimization and improvement of the production and handling of CORC conductors, to arrive at the highest level of performance possible. 


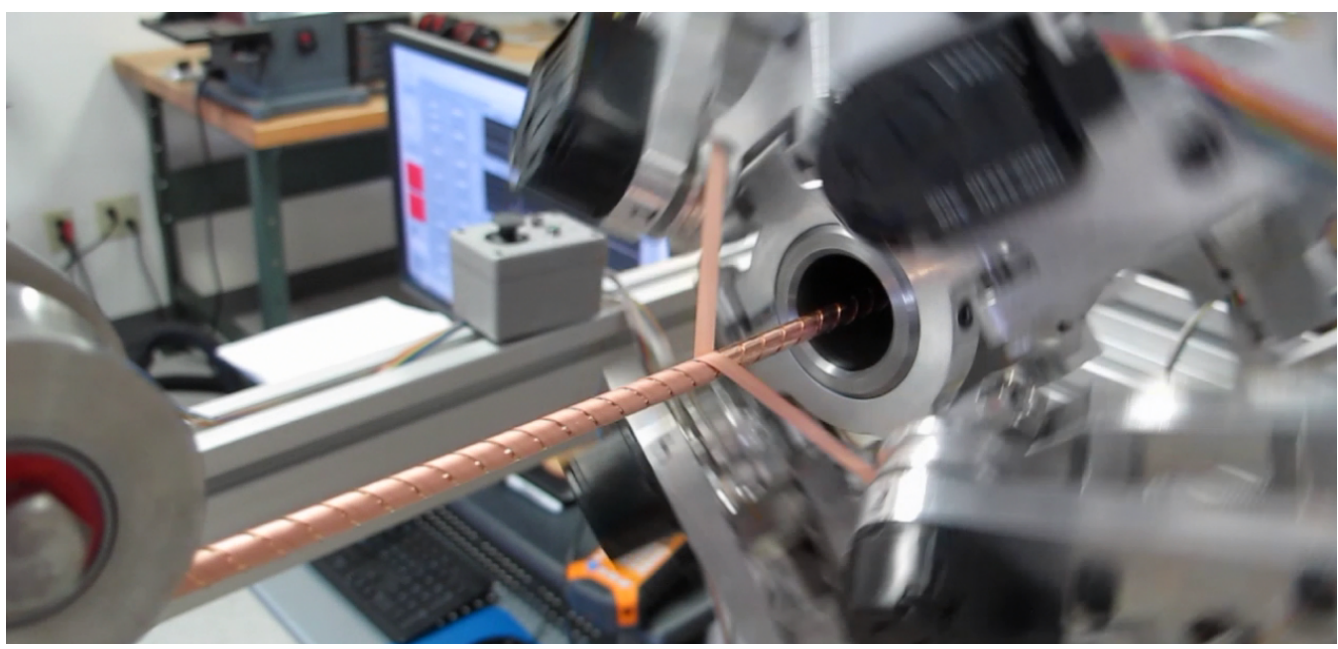

Figure 2.13: Picture of the CORC conductor winding machine of ACT during the layer-by-layer taping process [40].

\subsection{Conclusion}

In this chapter, the development route from a single ReBCO coated conductor to a multitape CORC conductor is described. ReBCO coated conductors are produced by over 10 different manufacturers using a wide scalar of production routes, which yields tape ranging from 2 to $12 \mathrm{~mm}$ in width, with a variety in internal substrate and stabilizer layouts and also in electrical performance.

Recent advancements in tape technology, such as the reduction of substrate thickness of SuperPower tape from 50 to $30 \mu \mathrm{m}$ and additional $\mathrm{Zr}$ doping significantly increased the tape's electrical performance significantly. It is shown that the reduction of substrate thickness is key in the development of CORC wire technology, as it causes an increase in overall tape, and thus CORC current density and also a reduction of the minimum diameter of CORC conductors. This development allowed preparation of CORC wires with diameters of 2.4 to $4 \mathrm{~mm}$ and a current density ranging from 400 to $800 \mathrm{~A} / \mathrm{mm}^{2}$ at $10 \mathrm{~T}$ and $4.5 \mathrm{~K}$.

One of the main features of CORC is its high tolerance to omni-directional bending, which is made possible by tapes sliding within the CORC conductor while bending. Still, the flexibility of CORC conductors is limited and highly dependent on conductor and tape layout. Optimization of the conductor design and production settings has to be performed on a case-by-case basis for optimal electrical performance and flexibility. 


\section{Chapter 3}

\section{Current Distribution in and Resistance of CORC Joints}

CORC joints are essential for the conductor's performance, as they have a dominant role in determining current distribution among ReBCO tapes and are a constant source of ohmic heating. Therefore, low-resistive joints, that feature a uniform current distribution are key. In this chapter, the state-of-the art joints for CORC that were available at the start of this work and their limitations are described. Thereafter, the physical aspects behind current sharing are explained. This is followed by the introduction of a novel conductor joint technique for CORC conductors that is investigated by ACT and CERN, which offers a significant improvement of current distribution among ReBCO tapes and a significant reduction in joint resistance compared to previous designs.

\subsection{Introduction}

Current sharing in superconducting multi-strand conductors has always been an important factor in their design. Injecting current homogeneously in each strand is important for stable thermal and electrical operation of the conductor. Inhomogeneous current injection leads to some strands operating closer to their current limit than others, making them more prone to quenching and, as side effect, generating an unwanted perturbation in magnetic field. Additionally, superconductors are precious materials, especially HTS $R e B C O$ tapes. Arguably, the wide spread use of ReBCO tapes is currently mainly limited by their high cost. Therefore it is beneficial to make optimal use of all strands, not only for conductor stability, but also for financial reasons.

The CORC conductor comprises many ReBCO tapes and each tape has a resistive Hastelloy substrate. As discussed in the previous chapter, this Hastelloy layer faces outwards with respect to the CORC conductor's core to minimize the strain in the ReBCO. Hence, the ReBCO tapes in the CORC conductor need to be unwound over the length of the joint section to make direct electrical contact to the ReBCO side of the tape. Consequently it 
is challenging to prepare low-resistive electrical connections to CORC conductors that are not bulky and inject or extract current homogeneously to or from each tape. Several iterations of CORC joint designs have been realized over recent years in search of an optimal jointing solution.

The first CORC test samples had simple joint terminals, consisting of a flat metal plate to which all tapes of the samples were soldered individually, as shown in Figure 3.1 [23]. The tapes were unwound to allow direct soldering of the tapes, with the ReBCO side of the tape facing the metal plate. Besides being bulky, this type of terminal is not ideal as it is not practical for CORC cables with many tapes. Nevertheless, this design provides a functional electrical connection that allowed to demonstrate the feasibility of the first CORC cables ever made.

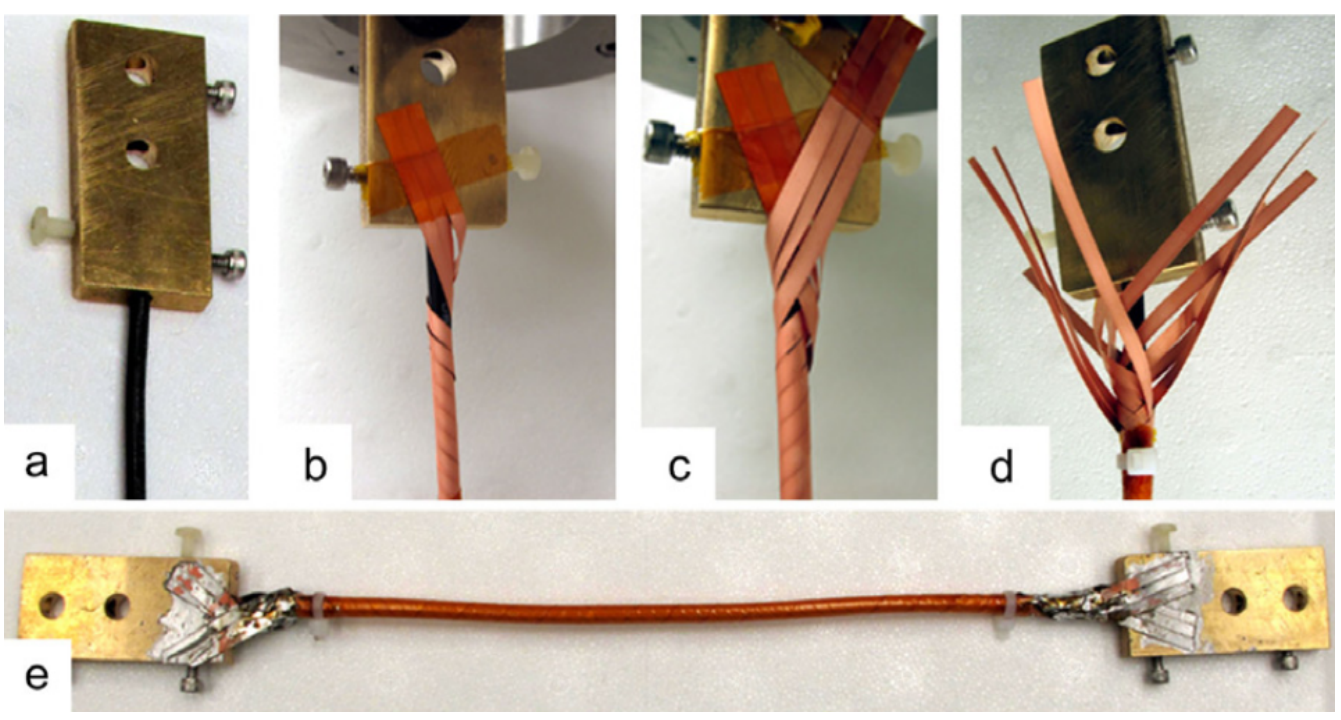

Figure 3.1: First type of joint terminals for CORC conductors, by which the ReBCO tapes are unwound and soldered individually to a flat copper or brass terminal [23].

The CORC joint design progressed from this flat terminal to a cone-shaped one. ReBCO tapes of the CORC cable's inner layers are soldered to the end of the cone and the tapes from the outer layers are soldered further towards the base of the cone. The cone shape allows to reduce the size of the terminal and allows jointing of CORC cables with more layers and tapes. However, this terminal type is still too bulky, not practical in preparation and can not ensure homogeneous current distribution over the different layers of the CORC cable [71, 72, 73]. The tapes soldered near the base of the cone have a lower contact resistance than tapes soldered to the end of the cone, purely due to the longer resistive path to the cone's tip, which made homogeneous current injection principally impossible as demonstrated in [72].

The next generation of CORC joint terminals were of the solder-filled type. The insulation is removed from the conductor and the tapes are partially unwound and inserted in a copper cylinder, as shown in Figure 3.2. The entire terminal is then heated and filled with 

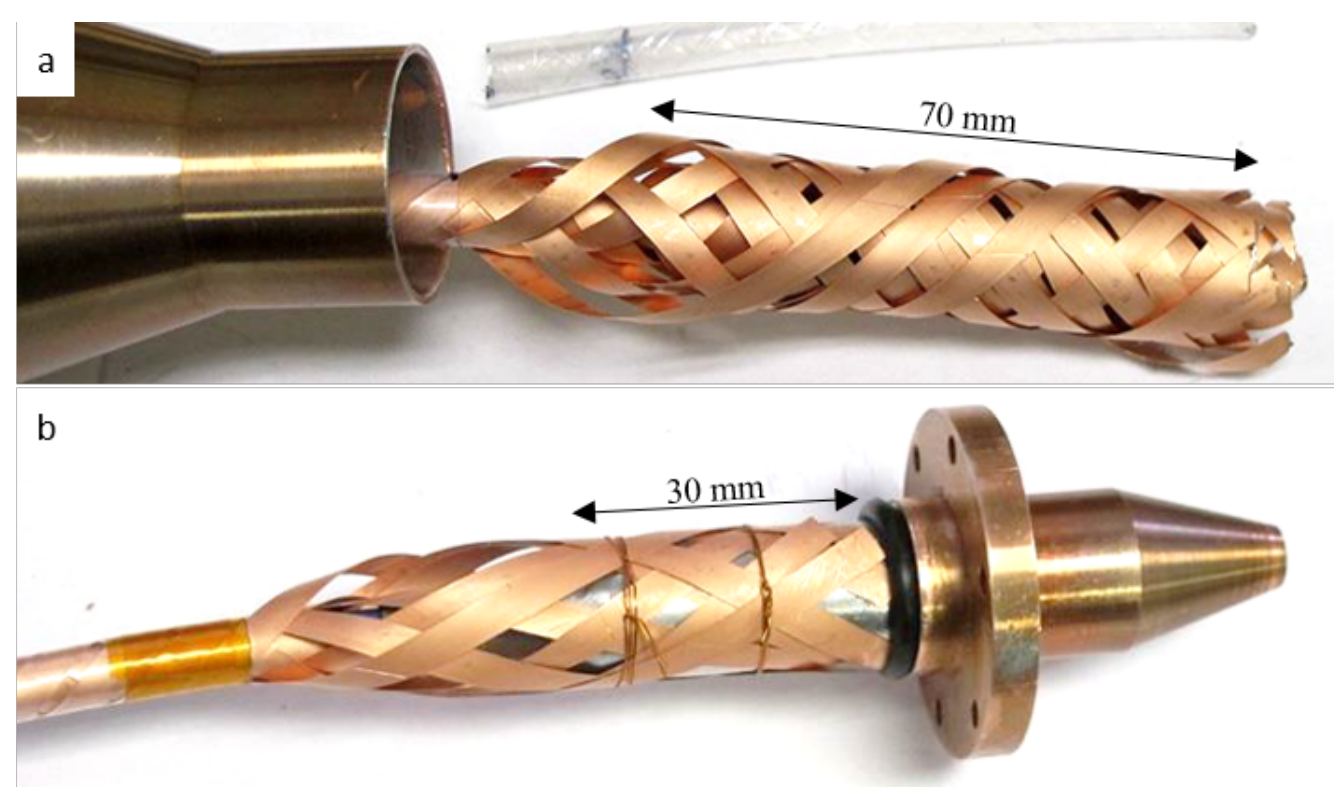

Figure 3.2: CORC joint terminal type in which the tapes are partially unwound and attached to a copper termination. A copper cylinder is placed over the tapes and filled with soldering material. The resulting resistance of this terminal is in the range of 6 to $111 \mathrm{n} \Omega$ [73].

solder. The solder can flow in-between tapes, since the tapes are partially unwound. The preparation is practical, however it yielded poorly reproducible joint resistance values ranging from 6 to $111 \mathrm{n} \Omega$ [73].

Another type of electrical joint is demonstrated and is shown in Figure 3.3. Here the insulation is removed from a section of a few centimeters of CORC cable. The tapes are pre-tinned without unwinding. A terminal is then clamped on the pre-tinned, un-insulated CORC cable. The resulting joint terminal has a resistance of some $250 \mathrm{n} \Omega$, which can be further decreased by increasing the length of the joint.

A complete overhaul in CORC joint design followed. The new terminal comprises a thin copper tube in which the end of the CORC cable is inserted. The voids between cylinder and cable are filled with solder, as shown in Figure 3.4. The resulting joint terminal has a similar size as the CORC cable itself and is practical in manufacturing, however, it does not distribute current well between layers. Current has to pass through the resistive Hastelloy of the outer layers first in order to reach the inner layers. Therefore, current flows predominantly in the outer layers since these have the lowest contact resistance. The current only reaches the inner layers at high overall current, when the outer layers are already saturated. A low joint terminal resistance and uniform current distribution was lost in favor of ease of preparation and reduced terminal size. This jointing design was the state-of-the-art at the start of the work described here.

A new solution to prepare electrical connections to CORC cables was therefore needed by which current homogeneously is injected in the ReBCO tapes and simultaneously the 

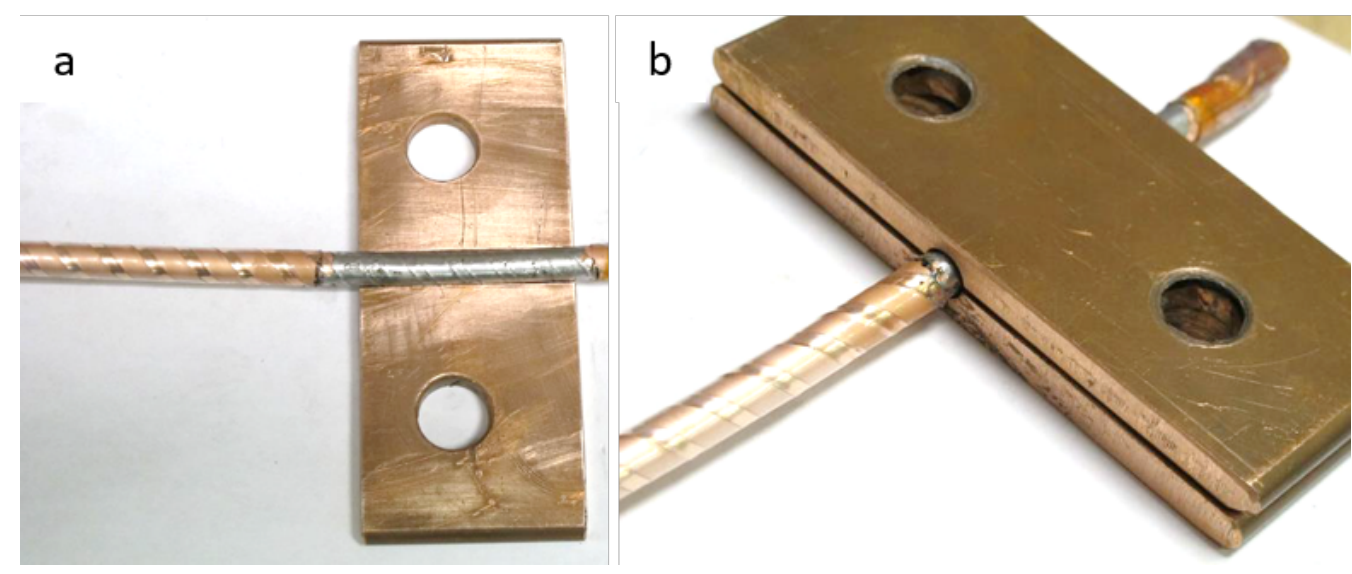

Figure 3.3: First joint terminal which does not require unwinding of the CORC cables. However, the resistance of these terminals are about $250 \mathrm{n} \Omega$ and the current is mainly injected in the outer layers [73].

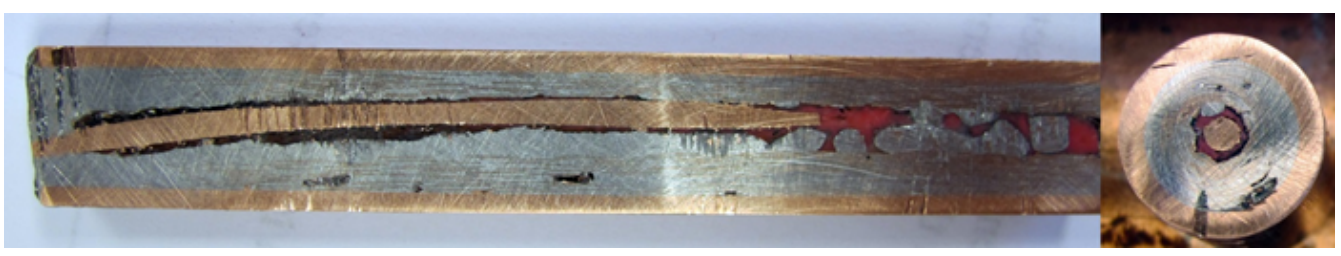

Figure 3.4: Longitudinal and transverse cross-sectional views of one of the first cylindrical tube terminals filled with solder. The core of the CORC cable is in the center of the tube and the tapes are spread out over the volume within the terminal. Some gas pockets are still present, especially near the insulated core [74].

resistance of these connections is reduced, while maintaining a practical preparation procedure.

\subsection{Physics Aspects of Current Sharing}

The thermal-electromagnetic stability of superconducting multi-strand conductors depends on several factors including available cooling, amount of stabilizer material, operating temperature, which impacts both temperature margin and heat capacity, and current sharing between strands. Current sharing between strands improves conductor stability, however it also increases coupling loss when changing current. There is a trade-off in conductor design when choosing optimal resistance values at various positions in the composite structure, which has to be fine-tuned for a particular conductor application. AC-operated applications, such as power transport cables, require a relatively high resistance between strands to minimize coupling loss. In DC-applications, such as most superconducting magnets, the design emphasis is generally on maximizing current shar- 
ing to ensure a homogeneous current distribution between all strands in a cable. However, even in DC applications there is an optimal inter-strand resistance, lower is not always better. Superconducting magnets always have to cope with varying magnetic field during a ramp to operating current, when switching off and often also during stationary operation under influence of external fields. Current (re)distribution among strands is driven by the electric potential difference between them. Taking the voltage reference $V=0$ on the current terminal, the potential of the $\mathrm{i}^{\mathrm{th}}$ strand can be written as:

$$
V_{i}=I_{i} R_{i}+U_{0}\left(\frac{I_{i}}{I_{c}}\right)^{n}+L_{i} \frac{d I_{i}}{d t}+\sum_{i \neq j} M_{i j} \frac{d I_{j}}{d t}
$$

The first term in this equation is the ohmic voltage across the strand-to-terminal contact resistance $R_{i}$, the second term is the usual power-law describing the superconducting nature of the strand itself and the last two terms are the strand's self-inductance and the mutual inductance between strands.

\subsubsection{Characteristic Tape Contact Resistance}

CORC type conductors comprise many ReBCO tapes in several layers. There is pressure contact between tapes of different layers. One tape is in contact with all tapes of the layer above and the layer below, i.e. a ReBCO tape in one of the inner layers of a CORC conductor with layers comprising each three tapes is in electrical contact with 6 other tapes. The inter-strand resistance between the ReBCO tapes in a CORC cable is about $1 \cdot 10^{-6}$ to $2 \cdot 10^{-6} \Omega \mathrm{m}$ [75], while the surface contact resistance $R_{s}$ per tape ranges from $2 \cdot 10^{-9}$ to $2 \cdot 10^{-7} \Omega \mathrm{cm}^{2}$, which translates to resistance as function of splice length $R_{L}$ of $R_{L}=R_{s} / W=5 \cdot 10^{-10}$ to $5 \cdot 10^{-8} \Omega \mathrm{m}$ per tape of $4 \mathrm{~mm}$ in width. The splice resistance of ReBCO tapes has been investigated extensively and a brief summary is presented in the Table 3.1.

The values presented in Table 3.1 are for splices prepared in a controlled environment specialized for preparing low-resistive tape-to-tape contacts. During preparation of the solder-filled state-of-the-art terminals for CORC cables, it is difficult and maybe even impossible to maintain a similar level of control. The tapes spring out slightly, but are not pressed against the tube's wall. Therefore, the amount of solder between the terminal's casing and ReBCO tapes is uncertain and can cause some variation in the tape-tocasing contact resistance; an additional $10 \mu \mathrm{m}$ of Sn63Pb37 solder at $4.2 \mathrm{~K}$ already adds $5 \mathrm{n} \Omega \mathrm{cm}^{2}$ to the splice resistance. Consequently it is important to quantify the effect of some variation in tape contact resistance on the current distribution within the CORC conductor. 
Table 3.1: Surface resistance of electrical connections to ReBCO coated conductor tapes at $4.2 \mathrm{~K}$ and $O$ T. The values provided correspond to only one ReBCO tape, thus a splice between two jointed ReBCO tapes has twice this resistance value.

\begin{tabular}{lclc}
\hline \hline & $\begin{array}{c}\text { Surface Resistance } \\
\left(\mathrm{n} \Omega \mathrm{cm}^{2}\right)\end{array}$ & Tape & Source \\
\hline ReBCO on the inside & 20 & SuperPower SCS4050 & {$[76]$} \\
CERN & 18 & SuperPower SCS4050 & {$[76]$} \\
CERN & 20 & SuperPower SCS4050 & {$[76]$} \\
CERN & 21 & SuperPower SCS4050 & {$[77]$} \\
University of Sao Paulo & 16 & SuperPower SCS12050 & {$[77]$} \\
University of Sao Paulo & $<20$ & SuperPower SCS4050 & {$[49]$} \\
SuperPower & 186 & SuperPower SCS4050 & {$[76]$} \\
ReBCO on the outside & 217 & SuperPower SCS4050 & {$[76]$} \\
CERN & & & \\
CERN & & & \\
\hline \hline
\end{tabular}

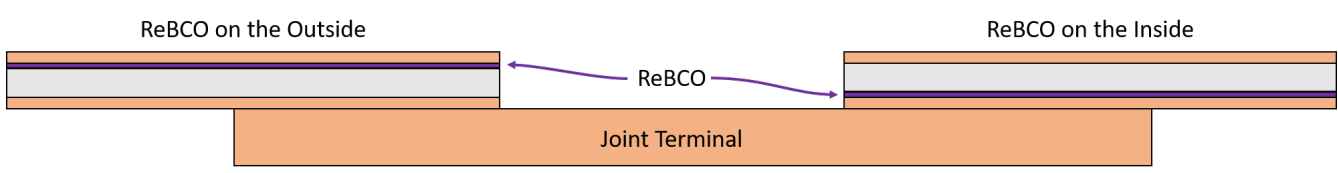

Figure 3.5: Two possible splice orientations when soldering ReBCO tapes to a metal terminal. The first option (left) is to splice with the ReBCO facing away from the terminal, thus with the resistive Hastelloy barrier in-between the superconductor and terminal. The second option (right) is with the ReBCO facing towards the joint casing.

\subsubsection{Current Sharing at Constant and Alternating Current}

\section{Effect of Tape Orientation on Current Distribution}

The joint terminals that were discussed in the introduction of this chapter can be divided into two basic categories: joints that inject current in the ReBCO side of the coated conductor, which requires unwinding of the ReBCO tapes, and terminals that inject current in the Hastelloy side of the coated conductor, which commonly does not require unwinding of the tapes. In the second type, the Hastelloy layer is situated between the ReBCO and the terminal's metal casing since the substrate in the tapes faces outwards. A common preconception, maybe even a misconception, is that soldering to the Hastelloy side of the coated conductor only brings disadvantages, due to the increased contact resistance and consequently the increased power dissipation in the joints. However, it also brings a benefit: the relative variation in splice resistance, e.g. due to a non-uniform solder layer thickness, is decreased as the absolute value of the splice resistance increases. This should result in a more uniform current distribution in the CORC cable. 


\section{Calculation of Current Distribution}

A relatively simple numerical model, based on Equation 3.1, is applied to calculate the current distribution in ReBCO tapes of a CORC cable as function of current for various contact-resistance configurations. Current sharing in DC-operated conductors with low ramp-rates is dominated by the resistance of the electrical connections and, for conductors of long length, also by the inter-strand resistance. Therefore the time-dependent part of Equation 3.1 can be neglected to find the current distribution in a DC-operated conductor. For short samples, the inter-strand resistance is much larger than the resistance of the electrical connections, so that it can be assumed that all current is distributed at the joint terminals. In this case the voltage over the sample is given by the following equation:

$$
V_{i}=I_{i} R_{i}+U_{0}\left(\frac{I_{i}}{I_{c}}\right)^{n} .
$$

Here $V$ is the voltage over the strand, $I_{i}$ is the current, $\mathrm{I}_{c}$ its critical current, $n$ the strand's $\mathrm{n}$-value and $R_{i}$ is the contact resistance between strand and terminal. The current in each tape is calculated by solving the following two equations:

$$
0=I_{i} R_{i}+U_{0}\left(\frac{I_{i}}{I_{c}}\right)^{n}-V_{i},
$$

and

$$
0=I_{t o t}-\sum_{i=1}^{n} I_{i} .
$$

The self-inductance and mutual inductance between either tapes or layers are added to Equation 3.3 allowing calculation of the time dependent current distribution (e.g. during current ramping):

$$
0=I_{i} R_{i}+U_{0}\left(\frac{I_{i}}{I_{c}}\right)^{n}+L_{i} \frac{d I_{i}}{d t}+\sum_{i \neq j} M_{i j} \frac{d I_{j}}{d t}-V_{i} .
$$

Here $L$ is the self-inductance and $M$ is the mutual inductance between strands. In addition, charge conservation demands that the total change in the overall current matches the sum of the current changes within individual ReBCO layers and the CORC's core:

$$
0=\frac{d I_{t o t}}{d t} \sum_{i=1}^{n} I_{i}
$$

The schematic representation of the electrical circuits for the constant and alternating current situations are presented in Figure 3.6. 
$\underline{\text { Constant Current }}$

$$
R_{c 1, \text { in }} \quad U_{0}\left(\frac{I_{1}}{I_{c 1}}\right)^{n_{1}} \quad R_{c 1, \text { out }}
$$

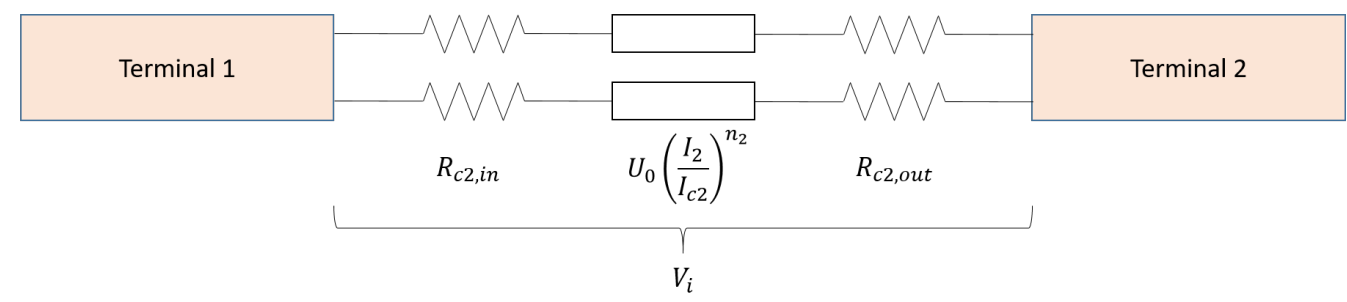

Alternating Current

$$
R_{c 1, \text { in }} \quad U_{0}\left(\frac{I_{1}}{I_{c 1}}\right)^{n_{1}}+L_{1} \frac{d I_{1}}{d T}+\sum_{j \neq 1} M_{1 j} \frac{d I_{j}}{d t} \quad R_{c 1, \text { out }}
$$

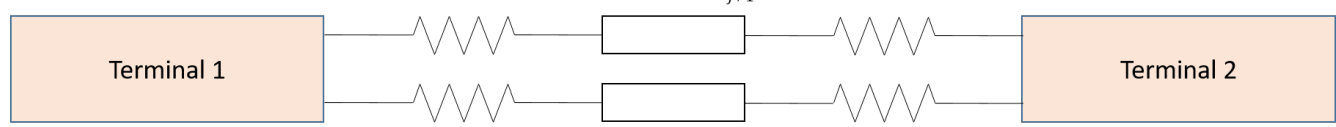

$$
\underbrace{R_{C 2, \text { in }} \quad U_{0}\left(\frac{I_{2}}{I_{C 2}}\right)^{n_{2}}+L_{2} \frac{d I_{2}}{d T}+\sum_{j \neq 2} M_{2 j} \frac{d I_{j}}{d t} \quad R_{C 2, \text { out }}}_{V_{i}}
$$

Figure 3.6: Electrical circuits used for calculating the current distribution among tapes within a CORC cable for a variety of contact resistances. In the figures, two tapes are represented, but the circuit can be scaled up to any number of tapes with each their individual contact resistance.

The following assumptions are made to demonstrate the difference in current sharing between directly splicing to the ReBCO side of the tape compared to splicing to the substrate side:

- A splice contact resistance of $20 \pm 5 \mathrm{n} \Omega \mathrm{cm}^{2}$ is assumed for splices to the ReBCO side of the tape, as illustrated in Figure 3.5. The error in splice surface resistance of $5 \mathrm{n} \Omega \mathrm{cm}^{2}$ is relatively high (and may actually be even higher in reality) as soldering a sizable amount of ReBCO tapes to a small joint terminal is a challenge where often precision is lost due to a lack of space.

- A splice contact resistance of $200 \pm 15 \mathrm{n} \Omega \mathrm{cm}^{2}$ is assumed for splices to the Hastelloy side of the tape, using the values reported in Table 3.1.

- All tapes are part of a CORC cable comprising 20 ReBCO coated conductors and a copper core of $5 \mathrm{~mm}$ in diameter.

The normalized current in the tapes with the highest and lowest contact resistances for both cases is presented in Figure 3.7. 


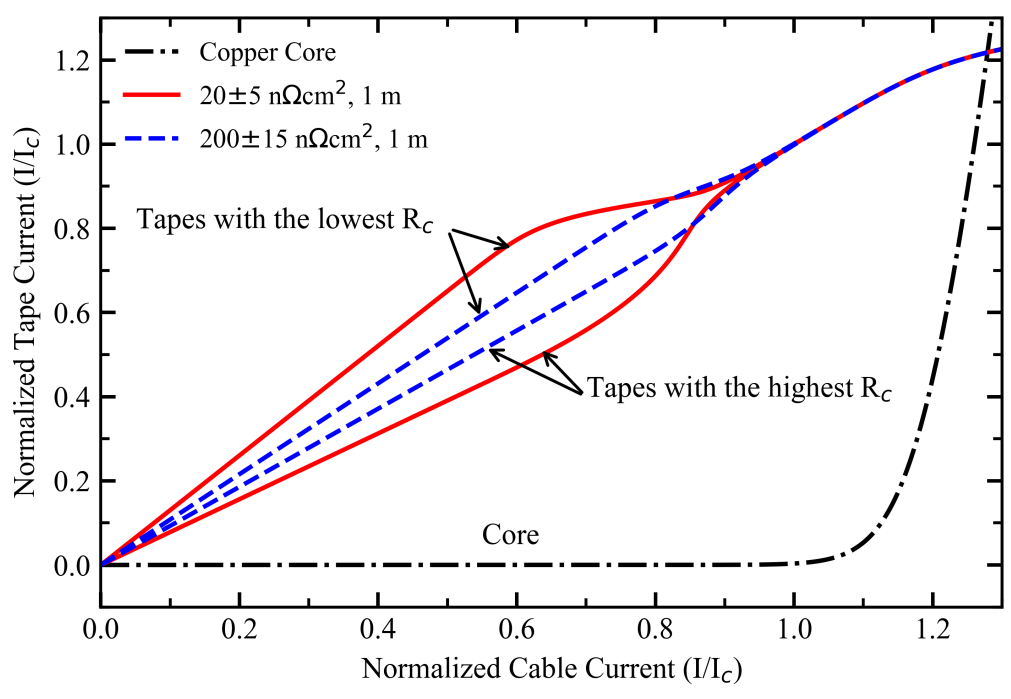

Figure 3.7: Model prediction of the normalized current distribution between the ReBCO tapes in a CORC cable that have the highest and the lowest contact resistances. Current primarily is in the tape with the lowest contact resistance, until that tape is saturated. A current increase beyond $I_{c}$ (after all tapes are saturated) pushes current to the conductor's stabilizer, in this case primarily into the CORC cable's copper core.

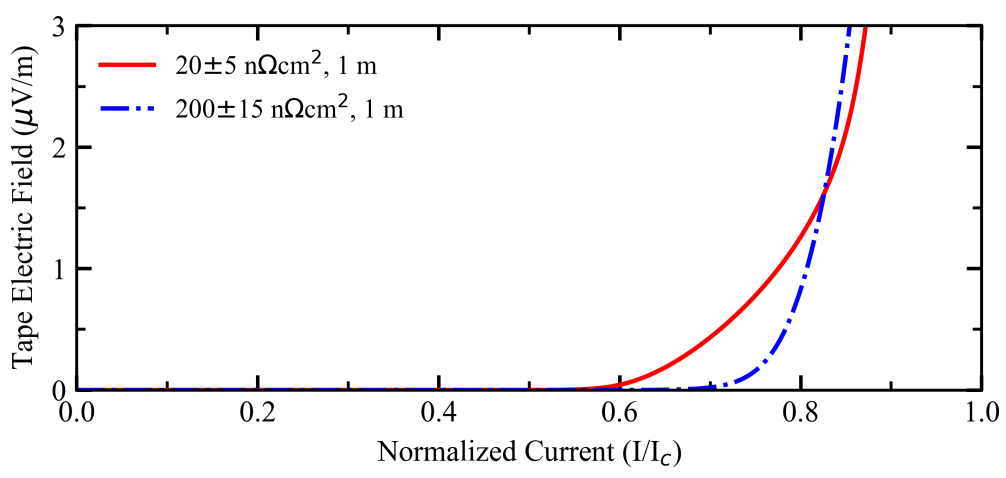

Figure 3.8: Modeled electric field in the tapes with the lowest contact resistance corresponding to the current distribution presented in Figure 3.7. In the case of a contact resistance of $20 \pm 5 \mathrm{n} \Omega$, the electric field (and thus Joule heating) rises at a lower current compared to the case of $200 \pm 15 \mathrm{n} \Omega$.

A low contact resistance with a relatively large uncertainty, which is the case with a resistance of $20 \pm 5 \mathrm{n} \Omega$, results in saturation of the tape with the lowest contact resistance at an overall current of about $60 \%$ of the cable $\mathrm{I}_{c}$ and this tape carries approximately $60 \%$ more current than the tape with the highest contact resistance. A similar distribution in 
current was experimentally observed in a CORC sample comprising 6 tapes in 2 layers, as is described in [72]. The current distribution between tapes improves when the Hastelloy substrate faces towards the splice, since the relative variation in the splice resistance is much lower compared to the splice of tapes soldered directly on the ReBCO side of the tape. In the case of a tape contact resistance of $200 \pm 15 \mathrm{n} \Omega$, the tape with the lowest contact resistance carries approximately $15 \%$ more current than the tape with the highest contact resistance. This improved current distribution causes a significant increase in the performance of the CORC cable, as the transition from the superconducting state to the normal state starts at a higher current and therefore less power is dissipated in the CORC cable in the current range of 60 to $80 \%$ of $\mathrm{I}_{c}$, as shown in Figure 3.8. The downside of having the Hastelloy substrate facing towards the splice is of course that it increases the joint resistance and thus increases the power dissipation in the joints. In both cases, the current is shared with the conductor's core when the tapes are nearing or exceeding their saturation current. Note how, at conductor $\mathrm{I}_{c}$, only a limited fraction of the current is shared with the copper core.

\section{Parametric Sweeps at Constant Current}

Three parameter sweeps were performed to evaluate the current distribution among the tapes as function of the tape's contact resistance, the variation in contact resistance and the conductor length:

- The variation in surface resistance is swept from $20 \pm 2 \mathrm{n} \Omega \mathrm{cm}^{2}$ to $20 \pm 5 \mathrm{n} \Omega \mathrm{cm}^{2}$ in steps of $1 \mathrm{n} \Omega \mathrm{cm}^{2}$.

- The absolute surface resistance is swept from $20 \pm 5 \mathrm{n} \Omega \mathrm{cm}^{2}$ to $200 \pm 5 \mathrm{n} \Omega \mathrm{cm}^{2}$ in steps of $60 \mathrm{n} \Omega \mathrm{cm}^{2}$.

- The conductor length is swept in logarithmic steps from 1 to 1000 meters.

All sweeps assume that current is only shared in the joints. The current in the tapes with the highest and lowest surface contact resistance is presented in Figures 3.9, 3.10 and 3.11. The current on the $\mathrm{x}$-axis is normalized to the overall critical current of the CORC cable and the $y$-axis represents the normalized current deviation from the average tape current.

From the calculations the following conclusions are drawn:

- For the purpose of current sharing, it is beneficial to increase the uniformity of the splices either by reducing the absolute variation in joint resistance or by reducing the relative variation in surface contact resistance (i.e. by increasing the total resistance).

- The length of the conductor only starts to matter when the tapes are nearing their critical current and built up a substantial voltage level.

- The calculations assume that contact resistances $R_{c i, i n}$ and $R_{c i, o u t}$ (see Figure 3.6) have the same values. When this is not the case, tapes have different potentials, thus 


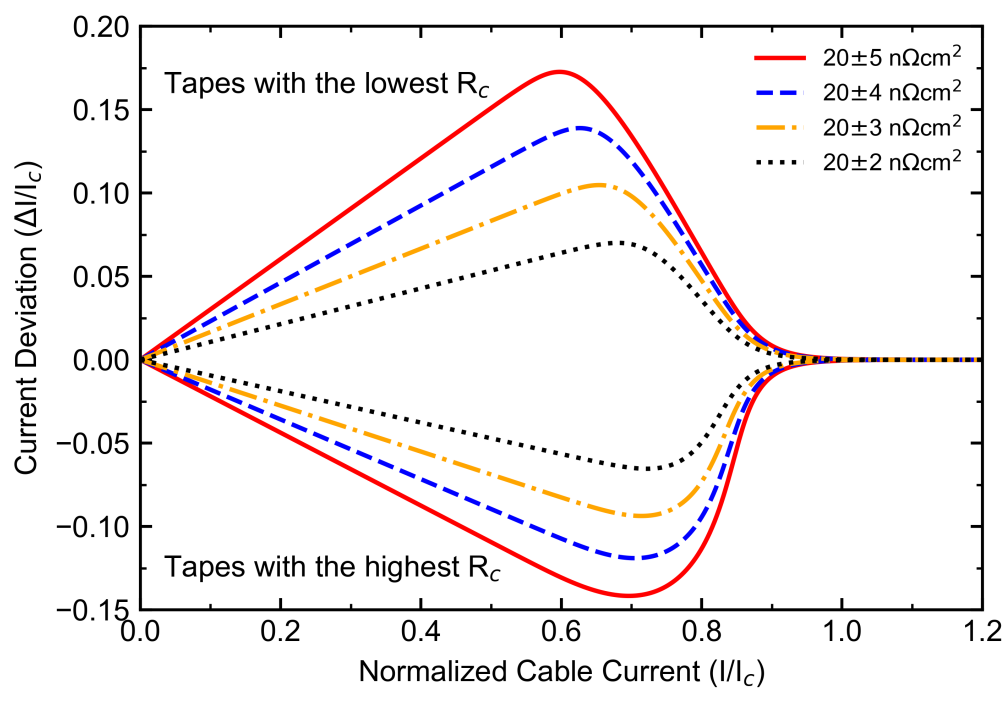

Figure 3.9: Calculated current in the ReBCO tapes in a CORC cable containing 20 tapes, plotted for both the lowest and highest surface contact resistance. Different pairs of curves correspond to different levels of variation around an average value of $20 \mathrm{n} \Omega \mathrm{cm}^{2}$.

current transfers from tape to tape. Consequently, in this case power is dissipated in the conductor at any current.

\section{Current Sharing at Alternating Current}

AC operation of CORC introduces extra inductive components to the current distribution within the conductor. AC operation as well as ramping up and down forces a current re-distribution between the layers of the CORC conductor and also in its metal core. The current distribution in short CORC samples still depends mainly on the contact resistance of each tape with the conductor joints, since the amount of coupled flux and hence, the inductive voltages are low. However, this is not the case in long CORC samples.

Similar to the DC case, the current distribution in an AC-operated CORC conductor may be calculated by solving equations $3.4,3.5$ and 3.6. The effects of inductive coupling are illustrated using a relatively straightforward example. The mutual inductance matrix of a straight CORC cable comprising 8 layers and a total 16 tapes is derived using ANSYS - MAXWELL and is used to calculate current distribution among layers as function of time. In Table 3.2, the values are presented for a conductor length of $1 \mathrm{~m}$. The mutual inductance values show that the layers are relatively strongly coupled, in the sense that they are comparable to the self-inductances. The table also illustrates how the mutual inductance values depend on layer winding direction, because the layers are not fully transposed within the CORC geometry. Layers with the same winding direction (e.g. layers 3 and 5) are better coupled compared to layers with the opposite winding direction 


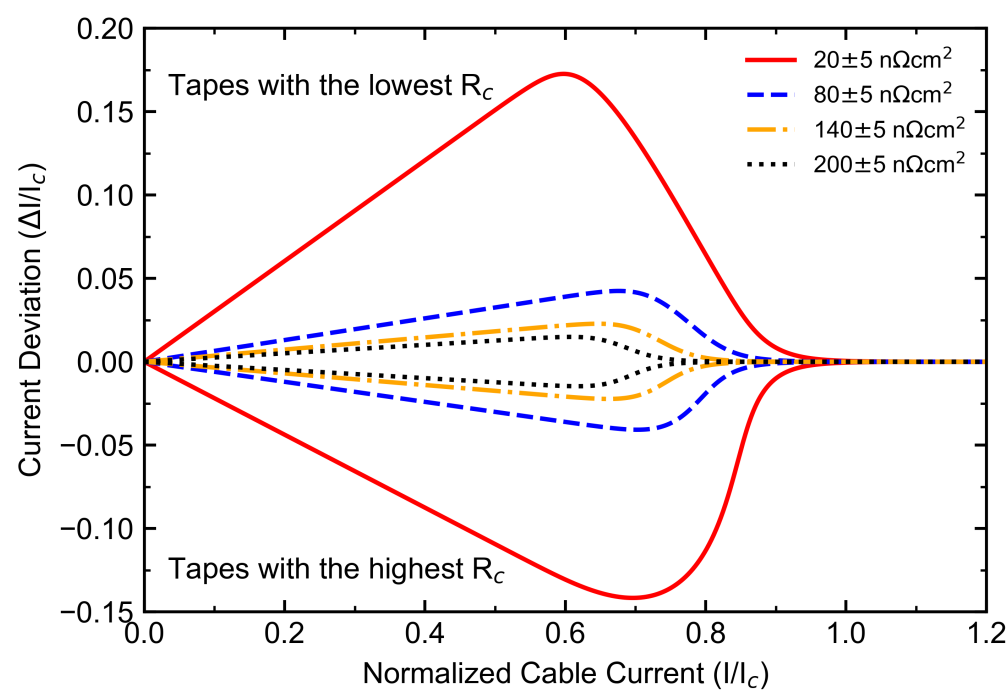

Figure 3.10: Normalized current in the tapes with the highest and lowest surface contact resistance as function of current. Between different pairs of curves, the contact resistance is varied without changing the variance of the resistance. Better current sharing is observed in the conductor with higher contact resistance, due to the higher consistency of the contact resistance.

(e.g. layers 3 and 4). The core of the CORC cable essentially behaves as a straight rod and has a relatively low self-inductance. It is also less coupled to the ReBCO layers.

With the self- and mutual inductance values in Table 3.2 known, an Ordinary Differential Equation (ODE) solver can work out the voltage drop across the cable and the individual layer currents as function of time. At low current, such that the voltage over the ohmic contacts is much lower than the inductive voltage, a variation in layer current of $5 \%$ is observed due to strong inductive coupling. At high current, such that the voltage over the ohmic contacts are similar to or exceeding the inductive voltage, the variation in layer current either increases or decreases towards the variation in the constant current case, as the voltage over the contacts becomes more dominant. The relative contribution of the joints is dependent on the overall current, conductor length, ramp-rate and layer layout.

\section{Power Dissipation in Conductor and Joints}

An important aspect of current sharing and joint resistance is the ensuing power dissipation. Power is dissipated in the electrical connections due to their resistance; in the conductor itself as its strands go through transition from superconducting to normal state; and between strands as current redistributes. Conductor test samples are often cooled directly by a surplus of liquid that provides a significantly larger cooling power than is required. Joints with a high resistance are then permitted as long they do not negatively 


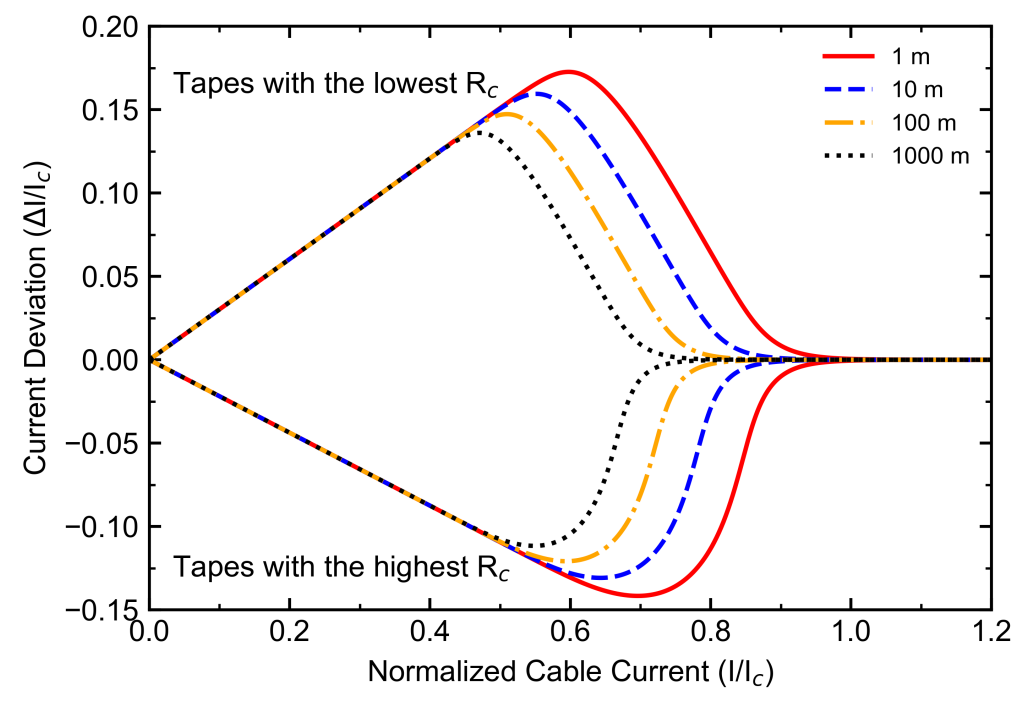

Figure 3.11: Normalized current in the tapes with the highest and lowest surface contact resistance as function of current. In this case, the conductor length is varied, keeping the surface contact resistance of each tape at $20 \pm 5 \mathrm{n} \Omega \mathrm{cm}^{2}$. A longer conductor length does improve current distribution when the first tapes start to saturate.

Table 3.2: Inductance matrix of an 8 layered CORC cable, all values are in $\mathrm{nH} / \mathrm{m}$.

\begin{tabular}{lccccccccc}
\hline \hline & Core & Layer 1 & Layer 2 & Layer 3 & Layer 4 & Layer 5 & Layer 6 & Layer 7 & Layer 8 \\
\hline Core & $\mathbf{4 3 . 2}$ & 82.7 & 82.2 & 81.7 & 81.2 & 80.7 & 80.3 & 79.9 & 79.5 \\
Layer 1 & 82.7 & $\mathbf{1 7 8 . 3}$ & 151.9 & 176.1 & 149.5 & 174.4 & 147.4 & 173.0 & 145.5 \\
Layer 2 & 82.2 & 151.9 & $\mathbf{1 7 9 . 1}$ & 149.0 & 177.0 & 146.9 & 175.2 & 145.0 & 173.9 \\
Layer 3 & 81.7 & 176.1 & 149.0 & $\mathbf{1 8 0 . 0}$ & 146.4 & 178.0 & 144.3 & 176.2 & 142.3 \\
Layer 4 & 81.2 & 149.5 & 177.0 & 146.4 & $\mathbf{1 8 0 . 9}$ & 143.8 & 178.8 & 141.8 & 177.1 \\
Layer 5 & 80.7 & 174.4 & 146.9 & 178.0 & 143.8 & $\mathbf{1 8 1 . 9}$ & 141.0 & 180.0 & 138.9 \\
Layer 6 & 80.3 & 147.4 & 175.2 & 144.3 & 178.8 & 141.0 & $\mathbf{1 8 3 . 1}$ & 138.1 & 181.1 \\
Layer 7 & 79.9 & 173.0 & 145.0 & 176.2 & 141.8 & 180.0 & 138.1 & $\mathbf{1 8 4 . 3}$ & 135.3 \\
Layer 8 & 79.5 & 145.5 & 173.9 & 142.3 & 177.1 & 138.8 & 181.1 & 135.3 & $\mathbf{1 8 5 . 9}$ \\
\hline \hline
\end{tabular}

influence the experiment. Actual applications and larger experiments on the other hand are often limited in cooling power and require-low resistive joints to keep the cost of cooling to a minimum.

The power dissipation is calculated for several CORC cable and joint configurations and the results are presented in Table 3.3. The tapes have a critical current of $300 \mathrm{~A}$ with an $\mathrm{n}$-value of 30 in a magnetic field of $10 \mathrm{~T}$ and the joint terminals are outside the magnetic field. The table shows that by increasing the variation in the joint resistance from $20 \pm 2$ to $20 \pm 5$, the power dissipation within the conductor increases by a factor of 2 at $80 \%$ of the conductor's $I_{c}$. The cable enters the current sharing regime due to inhomogeneous 
current distribution in the joint terminals already at $60 \%$ of the conductor's overall $\mathrm{I}_{c}$ value and a tape contact resistance of $20 \pm 5 \mathrm{n} \Omega$. The tapes are the dominant factor in the current distribution at conductor $\mathrm{I}_{c}$. At both $60 \%$ and $80 \%$ of $\mathrm{I}_{c}$, the current is better distributed over the tapes when the conductor length is increased. The power dissipated in the tapes is reduced by a factor 3 by increasing the conductor length from 1 meter to 10 meters. Further increasing the length does not reduce the power dissipation per meter of conductor significantly at 60 nor $80 \%$ of conductor $\mathrm{I}_{c}$. Increasing the contact resistance from $20 \pm 5$ to $200 \pm 5 \mathrm{n} \Omega$ causes a reduction in the power dissipation within the conductor itself with a factor 3 , but increases the total power dissipation by a factor 10 at both $60 \%$ and $80 \%$ of conductor $\mathrm{I}_{c}$.

The choice to increase the resistance of the electrical connections may be advantageous when cooling of the joints is more practical than cooling of the conductor. This allows operation closer to $I_{c}$ due to better current sharing and higher conductor stability. However, more efficient operation of the conductor obviously comes at a price of increased cooling requirements for the joint terminals.

Table 3.3: Calculated power dissipated in the electrical connections and within a $6 \mathrm{kA}$ CORC cable comprising 20 tapes, for several cable and joint configurations.

\begin{tabular}{|c|c|c|c|c|c|c|c|c|}
\hline \multirow[b]{2}{*}{ Run } & \multirow[b]{2}{*}{$\begin{array}{c}\text { Resistance } \\
\mathrm{n} \Omega\end{array}$} & \multirow[b]{2}{*}{$\begin{array}{l}\text { Length } \\
\mathrm{m}\end{array}$} & \multicolumn{2}{|c|}{ Power at $60 \%$ of $\mathrm{I}_{c}$} & \multicolumn{2}{|c|}{ Power at $80 \%$ of $\mathrm{I}_{c}$} & \multicolumn{2}{|c|}{ Power at $100 \%$ of $\mathrm{I}_{c}$} \\
\hline & & & $\begin{array}{c}\text { Joints } \\
\text { mW }\end{array}$ & $\begin{array}{l}\text { Conductor } \\
\mathrm{mWm}^{-1}\end{array}$ & $\begin{array}{c}\text { Joints } \\
\text { mW }\end{array}$ & $\begin{array}{l}\text { Conductor } \\
\mathrm{mWm}^{-1}\end{array}$ & $\begin{array}{c}\text { Joints } \\
\text { mW }\end{array}$ & $\begin{array}{c}\text { Conductor } \\
\mathrm{mWm}^{-1}\end{array}$ \\
\hline 1 & $20 \pm 2$ & 1 & 12.9 & 4E-04 & 23.0 & 0.9 & 36 & 597 \\
\hline 2 & $20 \pm 3$ & 1 & 12.8 & 1E-03 & 22.9 & 1.2 & 36 & 597 \\
\hline 3 & $20 \pm 4$ & 1 & 12.7 & 4E-03 & 22.8 & 1.6 & 36 & 597 \\
\hline 4 & $20 \pm 5$ & 1 & 12.6 & $2 \mathrm{E}-02$ & 22.7 & 2.0 & 36 & 597 \\
\hline 5 & $20 \pm 5$ & 10 & 12.7 & 9E-03 & 22.9 & 0.7 & 36 & 597 \\
\hline 6 & $20 \pm 5$ & 100 & 12.7 & 3E-04 & 23.0 & 0.6 & 36 & 597 \\
\hline 7 & $20 \pm 5$ & 1000 & 12.7 & 7E-05 & 23.0 & 0.6 & 36 & 597 \\
\hline 8 & $80 \pm 5$ & 1 & 52.4 & 2E-04 & 92.2 & 0.9 & 144 & 596 \\
\hline 9 & $140 \pm 5$ & 1 & 90.1 & $1 \mathrm{E}-04$ & 161 & 0.7 & 252 & 596 \\
\hline 10 & $200 \pm 5$ & 1 & 130 & 9E-05 & 230 & 0.6 & 360 & 595 \\
\hline
\end{tabular}

\subsubsection{Current Sharing in HTS Power Cables}

Power cables were the first large-scale practical application based on ReBCO tape technology. They are designed to operate in the electrical grid as underground transmission lines transporting energy more efficiently than copper or aluminium transmission lines. HTS power cables comprise multiple layers of ReBCO tapes wound around the cable's former. One of the design challenges is that current tends to stay in the outer layers due to the difference in layer impedance. The layout of CORC conductors is very similar to these power cables. Therefore both conductors face similar issues in terms of current sharing.

There are many examples in literature of optimization of the tape and layer layout in HTS power cables to reduce AC loss and improve current sharing. The main optimization chal- 
lenge in power cables is to enhance current sharing, which may be hampered by inductive coupling between layers. Current sharing can be improved by optimizing the winding angle and winding direction of the ReBCO layers, in combination with optimization of the contact resistance[78, 79]. One solution offered is to equalize the impedance between layers, which can be achieved by increasing the joint resistance [79], similar to the DC case described in Section 3.2.2.

HTS power cables commonly comprise 2 to 4 layers of ReBCO tapes for power transport and 1 or 2 ReBCO layers to shield the outside world from the cable's self-field. Insulation between the ReBCO layers in power cables is thick to allow multiple-phase power and are usually wound with a winding angle between 60 and 80 degrees, as illustrated by Figure 2.10. Layers in CORC conductors are wound directly on top of each other and are typically wound with an angle between 40 and 60 degrees to improve the conductor's flexibility and to cope with their much thinner core. Therefore, layers in CORC conductors have better coupling between layers than layers in power cables. Low coupling in singlephase power cables (most similar to CORC) results in current de-phasing between the different layers, demonstrated by Mukoyama et al. [79] and consequently related ACloss. The phase difference can be eliminated, by either optimizing layer winding angle or contact resistance, which reduces the differences in their impedance and causes a decrease of AC-loss with a factor up to 10 [80].

The ReBCO layers in CORC conductors are strongly coupled, as all have similar radii and pitch lengths. Therefore, the current peaks of the different ReBCO layers are synchronous. To demonstrate this, the current sharing inside a CORC cable comprising 16 tapes in 8 layers is calculated at $50 \mathrm{~Hz}$ using a sinusoidal wave with an overall current amplitude

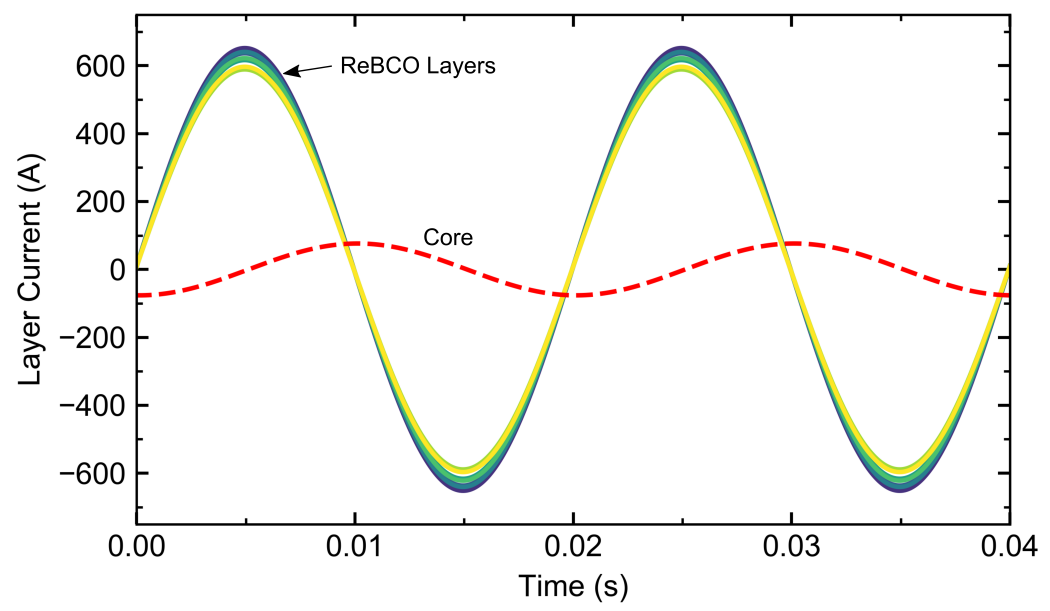

Figure 3.12: Current in the ReBCO layers and core as function of time for an alternating current with a frequency of $50 \mathrm{~Hz}$ and an amplitude of $5 \mathrm{kA}$. Current peaks in the ReBCO layers are synchronous with a variation of 5\%, while the core shows an a-synchronous peak and carries $1.2 \%$ of the total current. 
of $5 \mathrm{kA}$. The current distribution among the ReBCO layers is shown in Figure 3.12. The current is close to homogeneous maximum variation of 5\%. There is no de-phasing between current in the layers. The current in the CORC's core is not in phase with the current in the ReBCO layers and carries, in this setting, on average $1.2 \%$ of the total current, resulting in a significant ohmic loss.

CORC conductors offer much better initial current sharing due to their highly coupled layers compared to HTS power cables. However, a relatively large fraction of current is induced in the CORC's core. The resistivity of the copper core decreases as the temperature decreases, therefore causing a further increase of AC-loss. Therefore, CORC conductors, without a low-resistive core, have potential for application in single-phase power transport technology.

\subsection{Novel Joint Design}

\subsubsection{New Concept}

The main challenge of making 'good' electrical connections to CORC-type conductors is to inject current homogeneously in all layers of the conductor, while keeping ohmic loss acceptably low. State-of-the-art joint terminals for CORC cables at the start of this work were on the right track in terms of practical preparation and size, but were lagging behind in terms of current distribution and joint resistance. As discussed in Section 3.1, the preparation method for these terminals was to insert the CORC cable as a whole into a copper tube and to fill all voids between tube and conductor with solder. A novel approach to this preparation method was introduced by ACT to improve current sharing and to reduce joint resistance, while maintaining ease of preparation of the old design and a practical realization of this method was developed at CERN.

In the new method, the ReBCO tapes in the outer layers are cut short such that a staircase like geometry appears in the conductor's joint section before inserting it into the joint terminal, instead of simply removing the insulation material and inserting the conductor as a whole into the tube. This preparation method is called staging, also known as tapering, and each 'step' of the staircase is called a stage, as presented in Figure 3.13. The prepared CORC conductor can have multiple stages, with each containing one or more layers. The main idea behind this design is that current can flow directly to the exposed ReBCO tapes in the inner layers without having to pass the high-resistance Hastelloy and the buffer layers of the tapes in the outer layers. Consequently the contact resistance decreases and current is injected more homogeneously.

The design of the joint relies for a large part on the 'springiness' of the conductor's ReBCO tapes, but this springiness also poses a challenge during preparation. The tapes tend to spring out when the insulation material around the CORC conductor is removed. This can become problematic when the tapes spring out outside of the terminal, as they cannot be easily inserted into the terminal afterwards. However, when tapes spring out inside the terminal, they are pushed against the tube's inner wall, which reduces the solder layer 


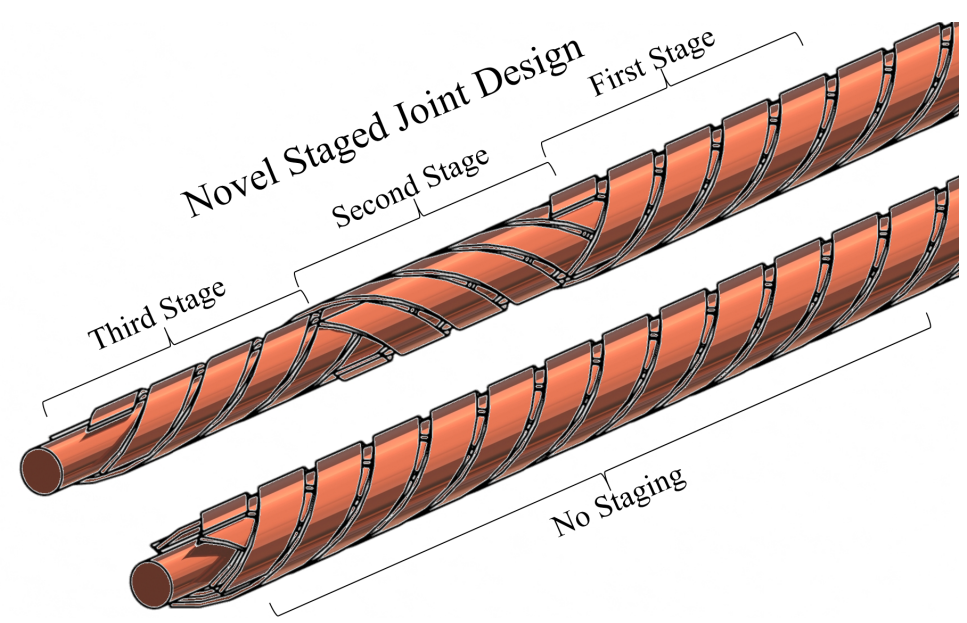

Figure 3.13: Image showing the new design with the staged CORC cable end compared to the old design without staging. The new joint design allows more direct injection of current into the inner ReBCO layers of the CORC cable by cutting away sections of tape creating a staircase-like geometry. The steps of this staircase are called stages and the cutting process is called staging, also known as tapering.

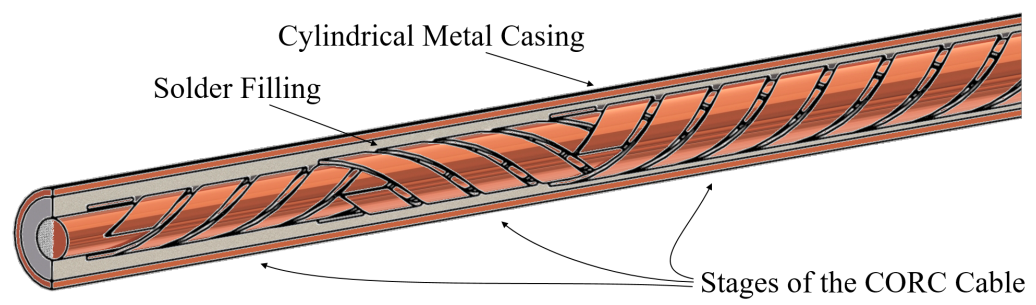

Figure 3.14: Image showing the staged CORC cable inside a cylindrical joint terminal filled with solder.

thickness between tape and terminal and thus reduces the contact resistance. Moreover, as the tapes spring out, the solder in the joint terminal can more easily flow to fill the voids between tapes. Figure 3.14 shows an image of a joint following the new design.

In support of this new joint approach, a custom-built nodal-network model is developed to help understanding the behavior of these joint terminals. The model demonstrates the benefits of the new jointing method when the old and novel designs are compared, but is also used to predict, evaluate and optimize joint configurations.

\subsubsection{Nodal-Network Joint Model}

The joint model is based on a network of 'resistors' that includes both linear- (solder and metal terminal) and non-linear components (ReBCO tapes), as shown in Figure 3.15. The model assumes a steady state situation, with constant currents so that inductive components can be ignored. 


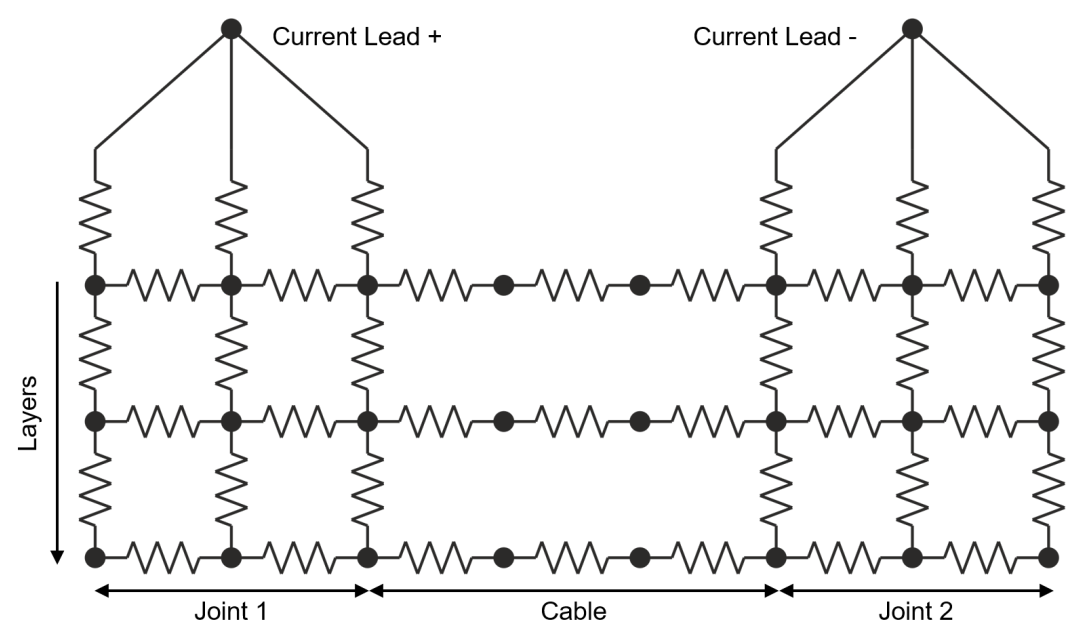

Figure 3.15: Schematic representation of a resistance network used for the calculations. Current can distribute between layers inside the joint terminal. It is assumed that there is no current transfer between layers in the CORC conductor as the contact resistance is there relatively high.

The model implements both Kirchhoff's voltage and current laws to solve the voltages in the nodes and the currents through the branches of the network. Kirchoff's voltage law expresses the single-valuedness of the electric potential by demanding that the sum of all voltage drops across the branches of any closed path through the network equals zero:

$$
\sum_{k=1}^{n} V_{k}=0
$$

Kirchhoff's current law states that the sum of the currents that flow into a node is equal to the sum of the currents that flow out of that node, thus expressing charge conservation:

$$
\sum_{k=1}^{n} I_{k}=0
$$

The system is solved for both Kirchhoff's laws for each branch between two nodes by applying the following equation:

$$
\left(V_{j}-V_{i}\right)-I_{i j} R_{i j}=0,
$$

where $V_{i}$ is the potential of each node, $I_{i j}$ the current that flows between them and $R_{i j}$ is the calculated temperature- and field dependent resistance. The equation is slightly rewritten for all connections that comprise ReBCO tape: 


$$
\left(V_{j}-V_{i}\right)-L U_{0}\left(\frac{I_{i j}}{I_{c}}\right)^{n}=0,
$$

where the second term includes the power-law description of the electric field - current relationship and the length $L$ between nodes. The nodes typically represent the actual ReBCO part of the the tapes, as indicated in Figure 3.16. The resistance between two nodes is calculated with the following equation, which includes both the current flowing in parallel through the solder and stabilizer material (the $1^{\text {st }}$ term) and the current going straight through the tapes (the $2^{\text {nd }}$ term):

$$
\frac{1}{R_{n}}=x\left(\frac{\rho_{p} \ln \left(r_{k} / r_{k+1}\right)}{2 \pi L}\right)^{-1}+(1-x)\left(\sum_{k=1}^{n} \frac{\rho_{k} \ln \left(r_{k} / r_{k+1}\right)}{2 \pi L}\right)^{-1} .
$$

Here $R_{n}$ is the total resistance from node to node, $\rho_{p}$ is the resistivity of the parallel 'bypass', $\rho_{k}$ is the effective resistivity of the materials within the ReBCO tapes and of the solder in-between the tapes, $r$ is the radial position of the node and $L$ is the length of the element. The natural logarithm takes into account the radial 'fanning in' of the current as it flows from a higher to a lower layer. Here, $x$ represents the relative cross-sectional area of the parallel bypass, which can be different for each CORC conductor configuration. The temperature dependent resistivities of copper and Hastelloy are taken from [81, 82] and the field dependence of the copper resistivity is taken from [83]. The solder resistivities are taken from [81, 84]. The bond between the silver layer and Hastelloy and between the silver layer and buffer layers is not perfect and therefore a significantly higher resisitivity than that of pure silver was used to model this cap layer [85].

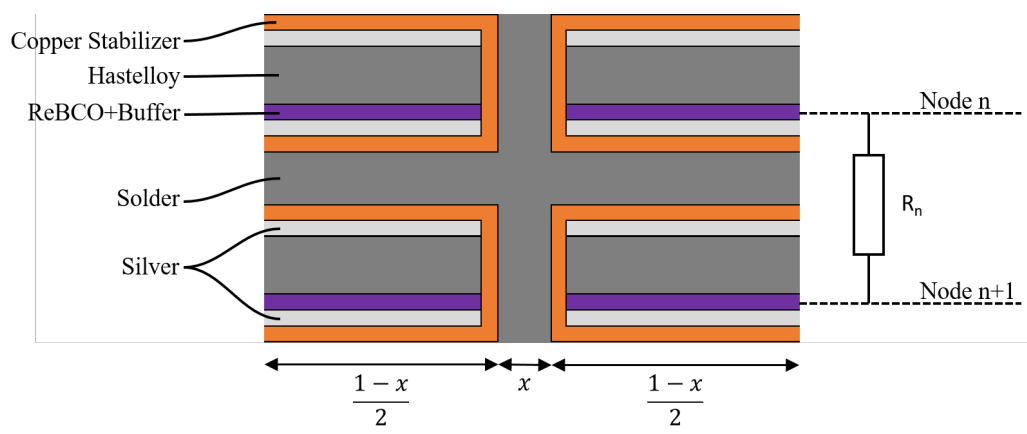

Figure 3.16: Schematic view of the different components in the tapes included in the joint model. Nodes are typically placed in the ReBCO layer of the tape. Current flows from tape to tape either directly through the highly-resistive Hastelloy and buffer layers or bypassing it via the copper and solder.

\subsubsection{Calculation of Current Distribution}

The primary goal of the model is to evaluate the difference in performance between a staged CORC conductor joint (the novel design) and the non-staged joint. In this compar- 
ison, the model primarily assumes a conductor geometry with 36 ReBCO SCS4050 tapes wound in 12 layers ( 3 tapes per layer) around a copper core of $5 \mathrm{~mm}$ in diameter. Detailed conductor and joint configurations are then varied to predict the current distribution and resistance of specific joint layouts.
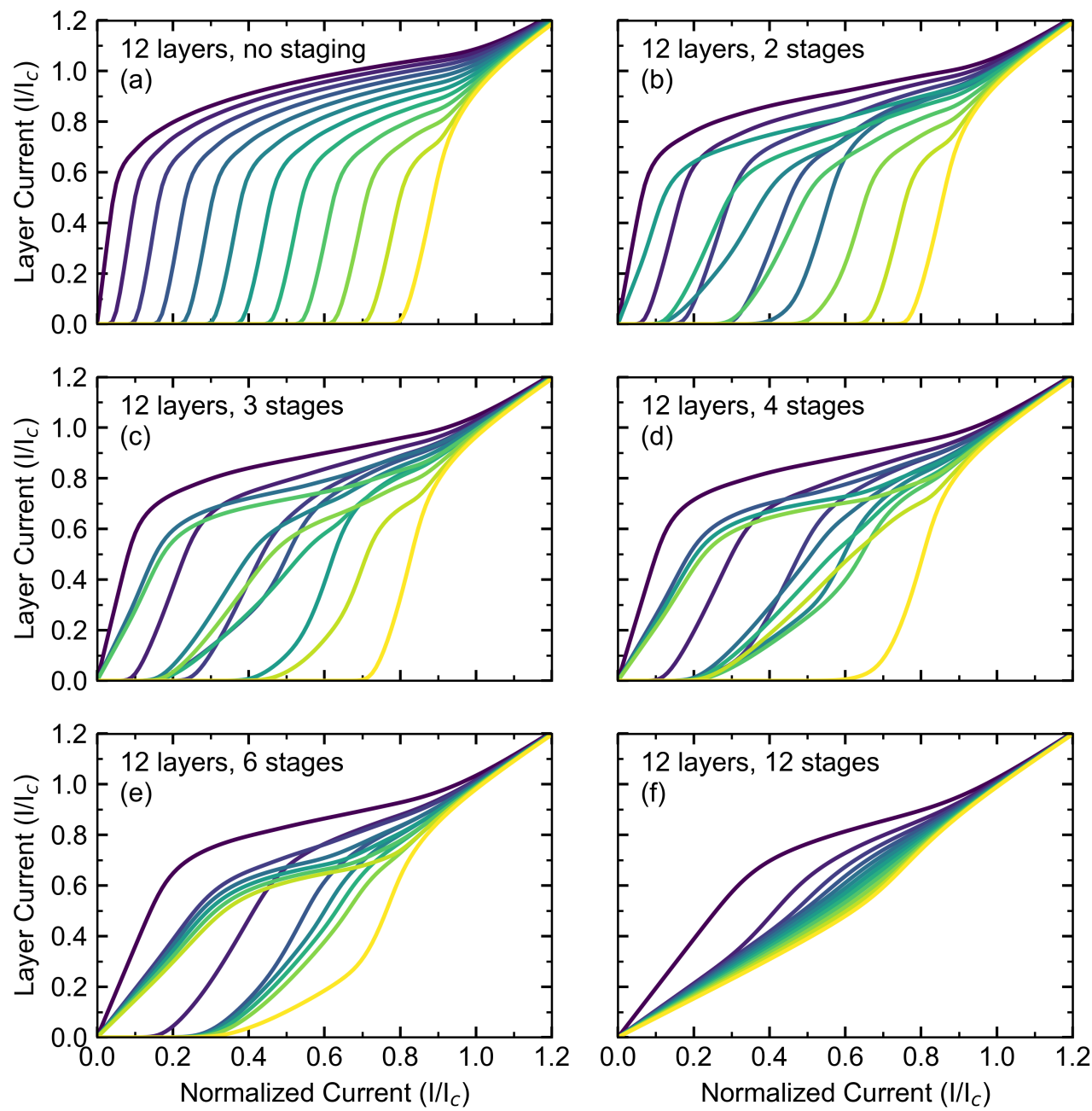

Figure 3.17: In Figure a to $f$ the current distribution in the different layers (different curves in each graph) is presented as function of the normalized current. The amount of stages has a large influence on the current distribution between layers of the CORC conductor. The different plots correspond to different stage configurations of a CORC cable with 12 layers. The stages are distributed equally over the joint length of $200 \mathrm{~mm}$. Staging each of the layers greatly improves the current distribution. The distribution of the fully staged cable (plot f) can be further improved by optimizing the individual stage lengths. 
The current distribution is calculated for the CORC cable using six different joint configurations. The joint configurations are varied representing the amount of stages, from 'non-staged' to a fully staged CORC cable. For the first comparison, the length of each stage is identical, distributed equally over the joint length. The joint is exposed to a magnetic field of $2.0 \mathrm{~T}$ at $5 \mathrm{~K}$. The calculated current distribution is presented as function of cable current for all six variations in Figure 3.17.

The non-staged configuration presents a clearly non-uniform current distribution. The outer layers first have to fully saturate with current before current starts to flow also in a deeper laying consecutive layer, since each consecutive layer has a higher contact resistance with the joint terminal than the layer before. Therefore, in this case, there typically is almost no current in the inner layers, while the tapes in the outer layers operate near their critical current. Each additional stage improves the current distribution in the cable substantially, up to the point where the cable ends are fully staged. However, the current distribution in the fully staged configuration is not yet fully homogeneous, since each stage has the same length as all other stages.

\subsubsection{Optimization of the Stage Length}

Staging each layer in the CORC cable to equal-length stages improves the current distribution compared to the non-staged case, as was illustrated in Figure 3.17. However, with equally sized stages, the current is not yet distributed perfectly homogeneous. Tapes in the inner layers do not spring out all the way to the bore-wall of the copper terminal. Therefore, the solder layer between the inner tapes and the terminal is relatively thick and thus these tapes have a higher contact resistance. The joint model is used to perform an iterative optimization of the stage lengths, aiming to create an equal contact resistance to each layer. As initial condition, all stages have the same length and in each iteration a percentage of the stage length of the layer with the lowest contact resistance is removed and added to the stage with the highest contact resistance. The variation $\left(I_{i}-\left\langle I_{i}\right\rangle\right) / I_{c}$, with $I_{i}$ the current in the $\mathrm{i}^{\text {th }}$ layer, $I_{\text {avg }}$ the average layer current and $I_{c}$ the cable's critical current, in the current distribution during this iterative solving process is presented in Figure 3.18.

The current in each layer of the CORC cable is well-distributed (within $2 \%$ of the average layer current) after 15 iterations. The corresponding optimized stage lengths for a $200 \mathrm{~mm}$ long CORC joint terminal are presented in Table 3.4. Note that this example is indicative, the exact distribution of stages along the length of the joint depends on the CORC conductor's tape layout and geometry and needs to be assessed for each individual conductor. A good rule of thumb for Sn63Pb37 solder-filled joints with typical CORC cables (using ReBCO tapes with a Hastelloy substrate thickness of $50 \mu \mathrm{m}$ ) that comprise at least 10 layers is to have the stage of the inner most layer approximately twice as long as the stage of the outer-most layer. 

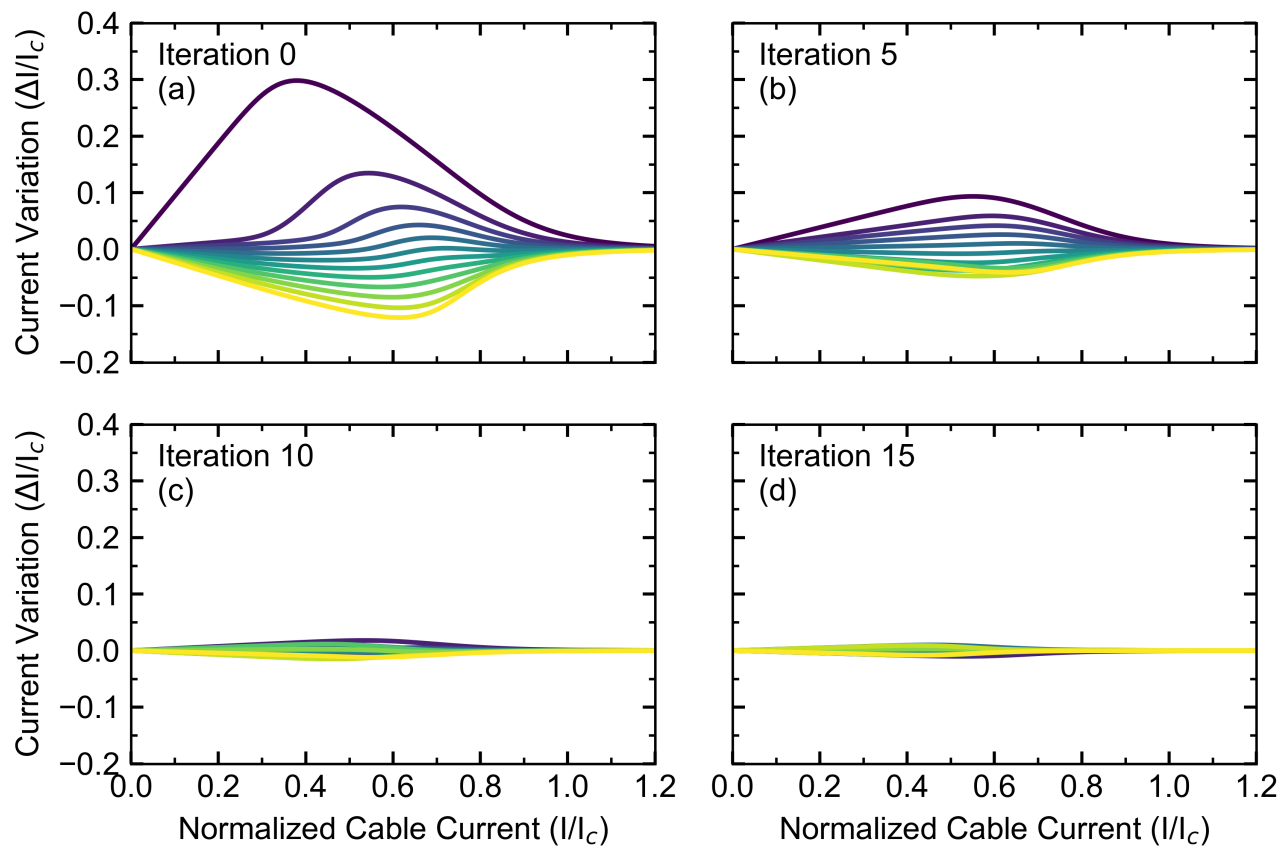

Figure 3.18: Variation $\Delta I / I_{c}=\left(I_{i}-\left\langle I_{i}\right\rangle\right) / I_{c}$ in tape current versus the normalized cable current during the iterative optimization process of the stage lengths. The initial condition uses 12 equal-length stages for a CORC cable of 36 ReBCO tapes distributed over 12 layers of 3 tapes each. The current in each layer is within $2 \%$ from the average layer current after 15 iterations.

\subsubsection{Calculation of Joint Resistance}

Low-resistance joints are preferred, since they limit the amount of joule heating and thus the amount of required cooling power. Staging inherently reduces the joint resistance, as a fraction of the highly resistive Hastelloy substrates is removed. The joint resistance as function of normalized cable current is calculated for various joint configurations and presented in Figure 3.19, once more for a joint of $200 \mathrm{~mm}$ in length that embeds a CORC cable comprising $36 \mathrm{ReBCO}$ tapes in 12 layers.

As introduced in Section 3.1, the joint resistance of the non-staged configuration starts already low at $6 \mathrm{n} \Omega$, but then quickly rises as successive layers saturate with current. This gradual saturation of successive layers shows up as the 'step-like' features in the non-staged curve in Figure 3.19. Staging increases the initial joint resistance, because the joint lengths of the outer layers are reduced due to the staging process. However, a clear reduction in joint resistance is observed at higher current, especially in the regime of 60 to $80 \%$ of cable $\mathrm{I}_{c}$. The reduction in resistance can be as high as $80 \%$ at $80 \%$ of the conductor $I_{c}$ when compared to the non-staged configuration. Staging the copper material of the terminal itself to match the shape of the staged CORC cable reduces the 
Table 3.4: Values for 12 optimized stage lengths in a $200 \mathrm{~mm}$ long joint terminal.

\begin{tabular}{lc|lc}
\hline \hline Layer & Stage Length $(\mathrm{mm})$ & Layer & Stage Length $(\mathrm{mm})$ \\
\hline Layer 1 & 12.2 & Layer 7 & 16.7 \\
Layer 2 & 14.6 & Layer 8 & 17.2 \\
Layer 3 & 15.1 & Layer 9 & 18.0 \\
Layer 4 & 15.6 & Layer 10 & 18.6 \\
Layer 5 & 16.2 & Layer 11 & 19.3 \\
Layer 6 & 16.4 & Layer 12 & 20.1 \\
\hline \hline
\end{tabular}

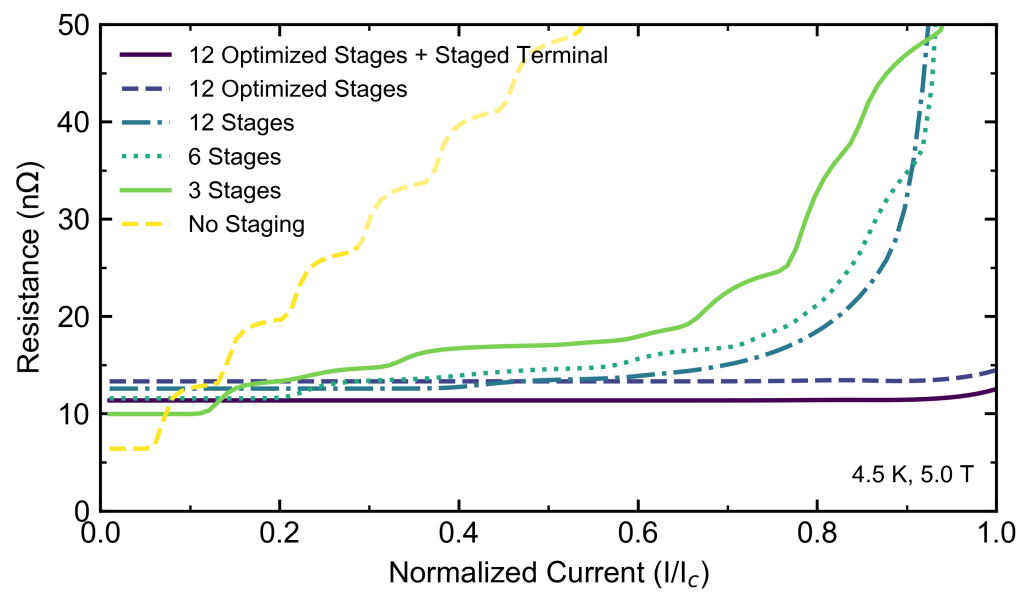

Figure 3.19: Joint resistance as function of normalized current for various staging configurations. The joint is $200 \mathrm{~mm}$ long, is filled with Sn63Pb37 solder and embeds a CORC cable comprising 36 ReBCO tapes in 12 layers. The joint resistance is reduced drastically by increasing the amount of stages to about $20 \%$ of its original unstaged value at $80 \%$ of the conductor's $I_{c}$. Optimizing the stage length and adding matching stages on the inside of the terminal's copper casing further reduces the joint resistance.

resistance of the joint terminal from $14 \mathrm{n} \Omega$ to $12 \mathrm{n} \Omega$. This reduction is mainly due to the replacement of solder by copper. Also, the tapes of the inner ReBCO layers don't have to spring out as far as before, because the inner bore of the terminal is closer to the tapes.

For each of the non-optimized joint configurations, a superconducting transition is observed well before the critical current of the CORC cable is reached. Inhomogeneous current distribution drives some layers into transition before others. The ensuing voltage build-up in the layer itself cannot be fully isolated from the joint voltage, which causes a steep rise in resistance when the cable $\mathrm{I}_{c}$ is approached. In the optimized joint configuration, such premature layer transition to the normal state has no effect on the joint resistance as all layers enter the transition simultaneously. 


\subsubsection{Parametric Sensitivity Analyses}

\section{Effect of Substrate Thickness on the Joint Resistance}

The Hastelloy substrate and the buffer layers in the ReBCO tapes forms a resistive barrier that more or less forces current to flow around it via the $\mathrm{Cu}$-stabilizer and the solder to the ReBCO side of the tape. Reducing the thickness of the Hastelloy substrate reduces the contact resistance of the tape. The effect of such reduction in substrate thickness on the joint terminal resistance is presented in Figure 3.20.

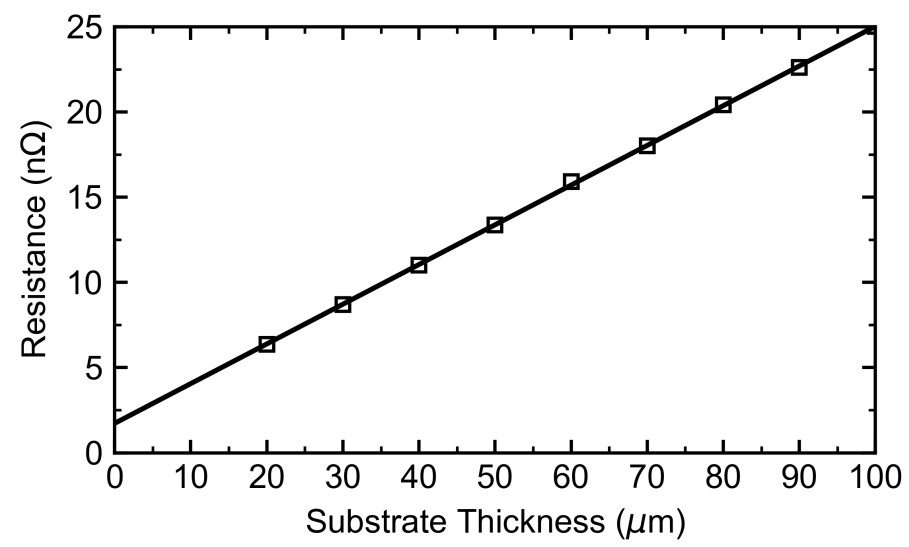

Figure 3.20: Joint resistance as function of tape substrate thickness for a joint of $200 \mathrm{~mm}$ in length. Reducing the Hastelloy substrate thickness reduces the joint resistance with $23 \mathrm{n} \Omega$ per $10 \mu \mathrm{m}$ of substrate. The Hastelloy layer still has a relatively high resistance compared to the parallel path of solder and copper, so that most current still flows around the substrate. The reduction in substrate thickness simply reduces the length of this parallel path and thereby reduces the joint resistance.

The joint resistance increases linearly with the substrate thickness. However, this effect is not caused by the reduction in resistance of the Hastelloy layer. The resistivity of the Hastelloy is several orders of magnitude higher than the resistivity of common solder metals. The thickness of the Hastelloy therefore hardly matters when there is a parallel path through the solder and Cu-stabilizer, as this parallel path will always carry most of the current. Instead, the reduction of Hastelloy substrate thickness also reduces the path length in the solder parallel to the Hastelloy, see Figure 3.16, and this is what causes the reduction in the overall joint resistance. CORC wires that use ReBCO tapes with only $30 \mu \mathrm{m}$ of Hastelloy substrate are therefore expected to have joints with a lower resistance than a CORC cable that uses ReBCO tapes with $50 \mu \mathrm{m}$ of substrate.

\section{Effect of Solder Resistivity on the Joint Resistance}

The standard solder used in the model and in the preparation of the terminals at CERN, described in Section 3.5, is eutectic tin-lead material (Sn63Pb37). This material is easy 
to use, commonly available, inexpensive and has a relatively low electrical resistivity of 5.4.10 $10^{-8} \Omega \mathrm{m}$ at $4.2 \mathrm{~K}$ outside magnetic field. Other materials, such as pure indium, have an even lower electrical resisivity of $1.8 \cdot 10^{-10} \Omega \mathrm{m}$, but are more expensive and less practical to use for filling voids as they have worse wetting properties than tin-lead. The resistivity of the solder has a relatively modest effect on the total joint resistance as shown in Figure 3.21. Replacing the tin-lead based solder by indium reduces the joint resistance by just over a factor 3 , despite the fact that the resistivity of indium is two orders of magnitude lower than that of the tin-lead eutectic.

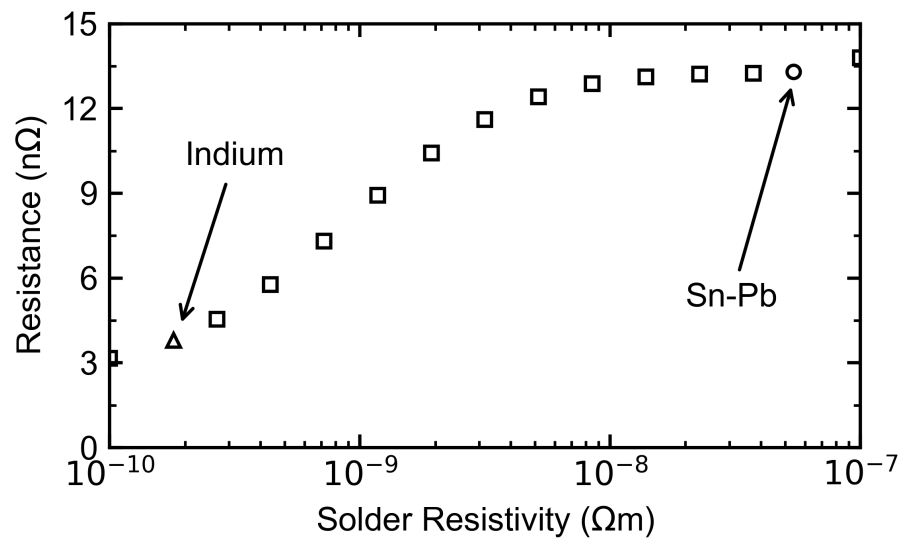

Figure 3.21: Resistance of a $200 \mathrm{~mm}$ long joint terminal as function of the solder resistivity. Replacing the tin-lead based solders by indium reduces the joint resistance by just over a factor 3 .

\section{Effect of Joint Length on the Joint Resistance}

Also, the length $l$ of the terminals effects their resistance, as illustrated in Figure 3.22. In this graph the calculated contact resistance is presented as a function of terminal length. The contact resistance decreases with increasing length of the terminal, following close to the expected $1 / l$ behavior as long as the ReBCO tapes within the joint terminal are still fully superconducting. This is no longer the case when the current inside tapes in the joint terminal is close to or over their critical current, because the tapes then start to behave similar to a normal conductor; increasing the terminal length in this case simply causes an increase in resistance. In an optimal scenario the joint terminals are exposed to a lower magnetic field than the main conductor. The current in such situation does not exceed or even come close to the overall critical current of the ReBCO tapes in the joint section and the joint resistance decreases essentially as $1 / l$ with increasing the joint length at any operating current. 


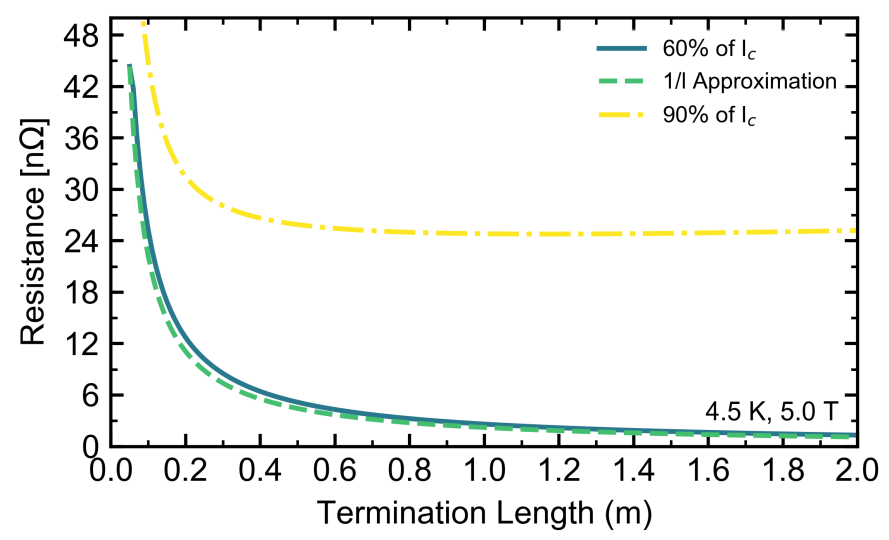

Figure 3.22: Joint resistance of a CORC conductor comprising 36 tapes in 12 layers as function of the joint length. The joint is in the same magnetic field as the main conductor section, which is a worst-case scenario. The joint resistance decreases with close to $1 / l$ when operated at $60 \%$ of the conductors $I_{c}$. Near or over $I_{c}$, the developing resistance of the ReBCO tapes causes an increase in the contact resistance with increasing length.

\subsection{Joints for CORC Cable-In-Conduit Conductors}

As described in Chapter 1, a CORC Cable-In-Conduit conductor comprises multiple CORC strands wound around a central rod or tube. Each CORC strand in this multi-strand conductor requires a low-resistance connection that distributed current homogeneously among strands. The design of the novel joint for single CORC cables, discussed in Section 3.3, is scaled up for application with CORC Cable-In-Conduit Conductors. A similar approach for staging and filling is used as for the joints of single CORC cables and wires.

The ends of each CORC strand are staged in order to improve current distribution among the layers in the strand and to reduce the joint resistance. The ReBCO tapes in each stage are held together with a bandage of solder, which melts during solder filling of the joint terminal. Although the CORC strands are twisted in the main conductor section to form the twisted cable bundle, the ends of the CORC strands cannot be twisted after applying the bandage of solder around the stages and it is impractical to twist the staged ends before applying this bandage. Therefore, the ends of the CORC strands remain straight over the joint length. The prepared ends of the CORC strands are bundled and placed inside a rectangular or cylindrical copper joint terminal, which is then filled with solder. A schematic layout of a joint terminal for a CORC Cable-In-Conduit Conductor is presented in Figure 3.23.

The resistance of joint terminals for the CICC conductors is calculated by combining the numerical joint model, described in Section 3.3.2, with a finite-element model in ANSYS. The numerical model incorporates the non-linear resistance of the superconductors and 


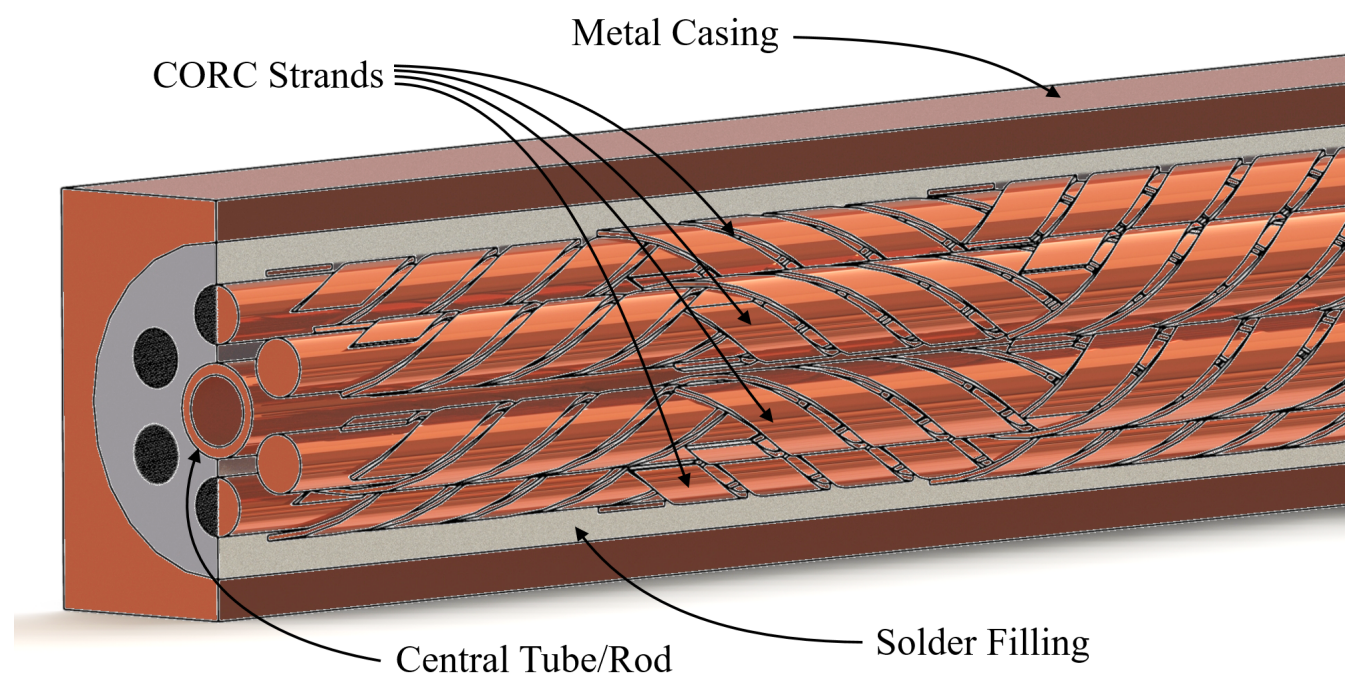

Figure 3.23: 'Cut-away' view of the joint terminal for a CORC CICC. Six CORC strands are bundled around a central rod or tube. Approximately $25 \%$ of the terminal's bore is filled with low-resistive solder.

the ANSYS model describes the bulk of the copper and solder around the bundle of CORC strands. A characteristic joint terminal for the CORC CIC-conductor is $350 \mathrm{~mm}$ in length and is predicted to have a resistance of about 1 to $2 \mathrm{n} \Omega$ at a temperature of $4.5 \mathrm{~K}$ in a magnetic field of $2 \mathrm{~T}$.

\subsubsection{Optimizing the Current Distribution Between CORC Strands}

The splice between two CORC CICC terminals or between a CORC terminal and a bus bar is often located only on one side of the joint terminal. Current is therefore only injected at one side of the joint terminal, which generally leads to inhomogeneous current distribution among the CORC strands. This is an effect especially important in relative short conductors. Each CORC strand has a different path length to the terminal surface where current is injected and thus experiences a different contact resistance. Strands located furthest from the splice have the highest contact resistance while strands closest to the splice have the lowest contact resistance. Current is shared accordingly.

A solution to improve the current distribution between strands is to have exactly a halfinteger twist pitch length between joint terminals, as shown in Figure 3.24. An N+0.5 number of pitch lengths between the terminals leads to a $180^{\circ}$ shift in strand layout inside the terminals. Strands that have a relatively low contact resistance in one joint then have a high contact resistance in the other and vice versa. In this case, each strand has almost the same total contact resistance, which results in a homogeneous current distribution 
between strands as shown in Figure 3.25. Here, the current distribution among strands is calculated for an integer and half-integer number of twist pitches between the terminals and plotted as a function of temperature.

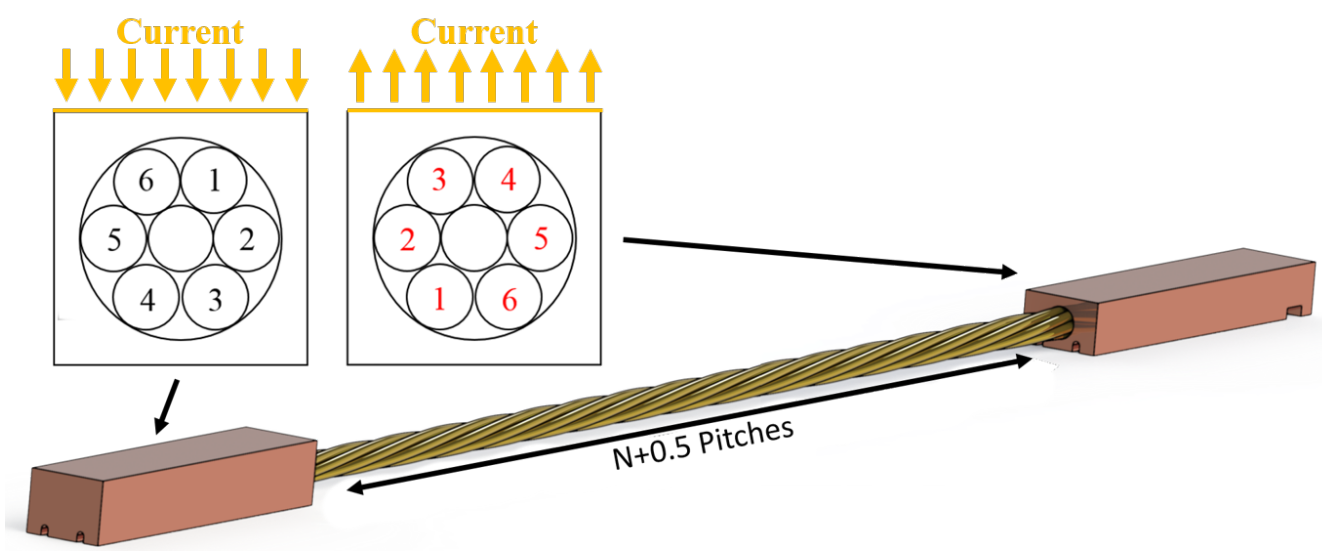

Figure 3.24: The bundle of six CORC strands requires a half-integer number of twist pitch lengths between the two joint terminals to reduce the variation in individual contact resistances to each strand on the current distribution within the cable.
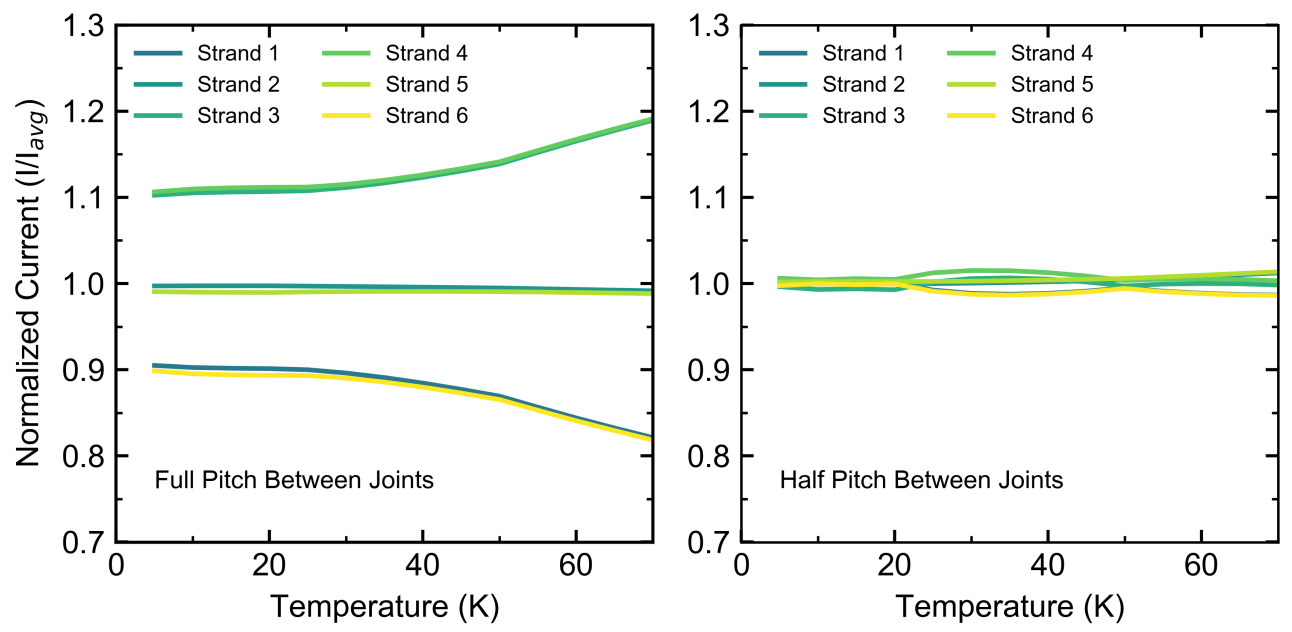

Figure 3.25: Calculated normalized current in each strand as function temperature for an integer and half-integer number of pitches in-between terminals. A half-integer number of pitches in-between the terminals provides a more homogeneous current distribution among strands, as each strand has roughly the same contact resistance. Strands that have a relatively low contact resistance in one joint have a high contact resistance in the other joint and vice versa. 
The operating temperature has a significant effect on the current distribution, since the resistivity of copper and of solder increases with temperature. At $4.2 \mathrm{~K}$, the contact resistance of the CORC strands is mainly dominated by the solder around- and the Hastelloy in the ReBCO tapes themselves. The resistivity of the copper in the terminals becomes a significant factor in the temperature range from 20 to $40 \mathrm{~K}$. Above $40 \mathrm{~K}$. the copper becomes even the main determining factor in the current distribution between strands.

Twisting the CORC strands also inside the terminal improves the current distribution. The best results are achieved with a full pitch in the terminal. The calculated current distribution as function of the ratio between pitch- and joint length is presented in Figure 3.26.

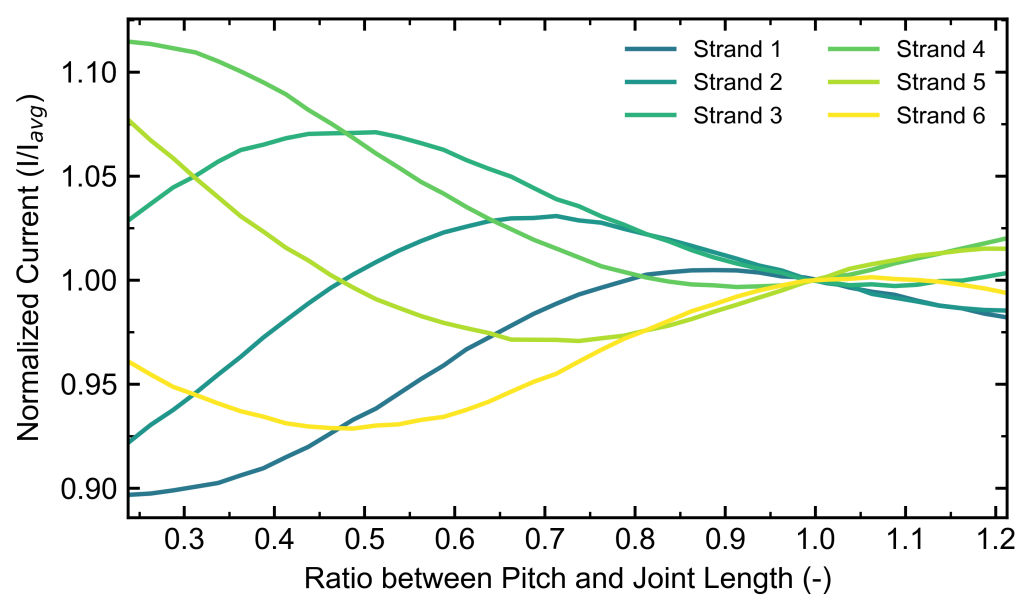

Figure 3.26: Calculated normalized current in each of the six strands as function of ratio between pitch- and joint length at a temperature of $77 \mathrm{~K}$. A joint length equal to the twist pitch length provides the most homogeneous current distribution among strands, as each strand experiences the same contact resistance. 


\subsubsection{Summary of the Modeling Results}

In summary:

- The lower resistance of splicing directly to the ReBCO side of the coated conductors might negatively affect the current distribution within the CORC cable, since the effect of small inhomogeneities in the joint become relatively more pronounced. This is also the case when reducing the joint resistance by using low-resistive indium solder or with ReBCO tapes with thin 20 or $30 \mu \mathrm{m}$ substrates.

- The non-staged CORC cable has the lowest resistance for currents below $10 \%$ of the conductor $\mathrm{I}_{c}$. In such joints, the outer layer still has its full length over the entire joint section and this layer is closest to the terminal's inner surface. Current flows into deeper layers only after the outer layer is fully saturated and starts to build up a voltage. The overall joint resistance increases when the current is increased and has to flow deeper and deeper in to the conductor in order to reach the non-saturated tapes.

- Staging the CORC conductor increases the contact resistance at low operating current since the outer layers are shortened and their contact surface is reduced. However, current can now flow directly to the inner layers, which reduces the joint overall resistance by $80 \%$ at $80 \%$ of the cable $\mathrm{I}_{c}$ and also improves current distribution. The joint resistance can be further reduced by matching the shape of the terminal to the shape of the staged CORC cable.

- The staged joint has a characteristic joint resistance of about $2.8 \mathrm{n} \Omega \mathrm{m}$ for a CORC cable with 36 ReBCO SCS4050 tapes in 12 layers at $4.5 \mathrm{~K}$.

- Thinner substrates reduce the joint resistance by reducing the path length to the ReBCO side of the tapes. Most current still flows around the substrate since the resistance of the parallel path with copper and solder is much lower than the resistance of the substrate.

- Replacing tin-lead solder by indium reduces the joint resistance by a factor 3 . The total joint resistance can be reduced by $80 \%$ by reducing the substrate thickness from 50 to $30 \mu \mathrm{m}$ and by using indium as solder material instead of tin-lead, as summarized in Table 3.5.

- The new joint design allows for practical up-scaling of the terminals for use with a CORC CICC. Hereby, it is important for uniform current distribution among strands to have half-integer number of cable pitches between the terminals. 
Table 3.5: Normalized joint resistance for various substrate thicknesses and two solder materials.

\begin{tabular}{lcc}
\hline \hline & \multicolumn{2}{c}{ Normalized Resistance $\left(\mathrm{R} / \mathrm{R}_{0}\right)$} \\
Substrate Thickness $(\mu \mathrm{m})$ & Tin-Lead & Indium \\
\hline 50 & 1.00 & 0.29 \\
40 & 0.82 & 0.24 \\
30 & 0.65 & 0.19 \\
20 & 0.48 & 0.14 \\
\hline \hline
\end{tabular}

\subsubsection{Engineering Values}

An indication of joint resistance as function of joint length is provided here for a typical CORC wire, cable and cable-in-conduit conductor. Note that these numbers merely provide a rough first estimate of the expected joint resistance. The actual resistance of a given CORC joint will depend on its layout and on the layout of the cable, which will result in some variation around the given values. The values are calculated using the nodalnetwork model. The model assumes that the CORC conductor in the joint is fully staged and that the entire joint is at $4.2 \mathrm{~K}$.

The resistance $R$ as function of joint length $L$ of a cylindrical joint terminal of a CORC wire of 30 SCS2030 tapes in 12 layers and an outer diameter of $3.5 \mathrm{~mm}$ is:

$$
R_{\text {wire }}(L)=1.8 \cdot 10^{-9}[\Omega \mathrm{m}] / L[\mathrm{~m}] .
$$

The resistance of a cylindrical joint terminal of a CORC cable of 36 SCS4050 tapes in 12 layers and an outer diameter of $7.6 \mathrm{~mm}$ is:

$$
R_{\text {cable }}(L)=2.8 \cdot 10^{-9}[\Omega \mathrm{m}] / L[\mathrm{~m}] .
$$

The resistance of a rectangular joint terminal of a six-around-one CORC CICC using CORC strands of 36 SCS4050 tapes in 12 layers and an outer diameter of $7.6 \mathrm{~mm}$ is:

$$
R_{C I C C}(L)=0.6 \cdot 10^{-9}[\Omega \mathrm{m}] / L[\mathrm{~m}] \quad \text { (single side injection). }
$$

Here it is assumed that current is injected to and extracted from only one side of the terminal. If current is injected to and extracted from two sides of the terminal, the resistance is:

$$
R_{C I C C}(L)=0.4 \cdot 10^{-9}[\Omega \mathrm{m}] / L[\mathrm{~m}] \quad \text { (double sided injection). }
$$

The resistance of CORC wire and cable terminals increases with a factor 3 when going from 4.2 to $77 \mathrm{~K}$. The resistance of the CORC CICC terminal increases with a factor 6 for single side injection or a factor 4.5 for injection on two sides of the rectangular terminal when going from 4.2 to $77 \mathrm{~K}$. 


\subsection{Experimental Realization of the Novel CORC Joints}

The practical realization of the new joint design comprises two main steps; the first is the staging of the ends of the CORC conductor and the second is the electrical and mechanical connection of the copper terminal to these ends. The detailed methods used during these steps are presented in this section.

\subsubsection{Preparation of CORC Conductor Ends}

The ReBCO tapes of the CORC conductor tend to spring outwards once the external conductor insulation is removed and thus the tapes have to be held in place during this preparation step. The tapes are fixed first at the cable ends (i.e. the last 10 to $20 \mathrm{~mm}$ of the conductor) before the insulation is removed. Fixing of the tapes is performed either with some Teflon tape tightly wrapped around the ends or by saturating the cable tip with solder.

The first stage consists of wrapping a thin enameled copper wire tightly around the CORC conductor at the boundary between successive stages, such that it holds the tapes within the marked section in place. A section of tapes is then staged by precisely cutting away the individual tapes between the copper wire and the conductor end. The optimal diameter for the copper enameled wire is $\sim 0.5 \mathrm{~mm}$. Thicker wires cannot be tightened enough to keep the ReBCO tapes in place and thinner wires tend to break during tightening. The marking and cutting of stages is then repeated for all the subsequent stages.

The wires are removed and the prepared CORC conductor is inserted into the copper tube terminal. However, this method proved to be impossible with a staged terminal that comprises a matching multi-stage bore, since the tapes spring out immediately when the thin copper wires are removed. A solution for this issue is to apply a bandage of solder around the stages of the CORC conductor. The solder holds the tapes in place during preparation and while inserting the conductor into the terminal's bore. An additional benefit is that the tapes are thus pre-tinned, which facilitates later solder-filling of the terminal. The bandage of solder melts when the tube is heated and the tapes are still able to spring out. A picture of the joint section of a prepared CORC conductor including the solder bandage is shown in Figure 3.27.

\subsubsection{Terminals for Single CORC Conductors}

The terminals for the CORC conductor either can be made cylindrical (tube) or rectangular. Rectangular terminals are more elegant for connections to other flat-surfaced objects, i.e. Rutherford type cables or bus bars. The draw-back of rectangular terminals is that they have to be oriented quite carefully around the CORC conductor. Otherwise there is a chance that the terminal does not align with the object it has to be attached to and consequently the CORC cable has to be twisted in order to align the terminals. The ensuing torque causes mechanical stress in the tapes, which can cause degradation by either buckling or straining the tape over its degradation limit, as discussed in Chapter 2. 


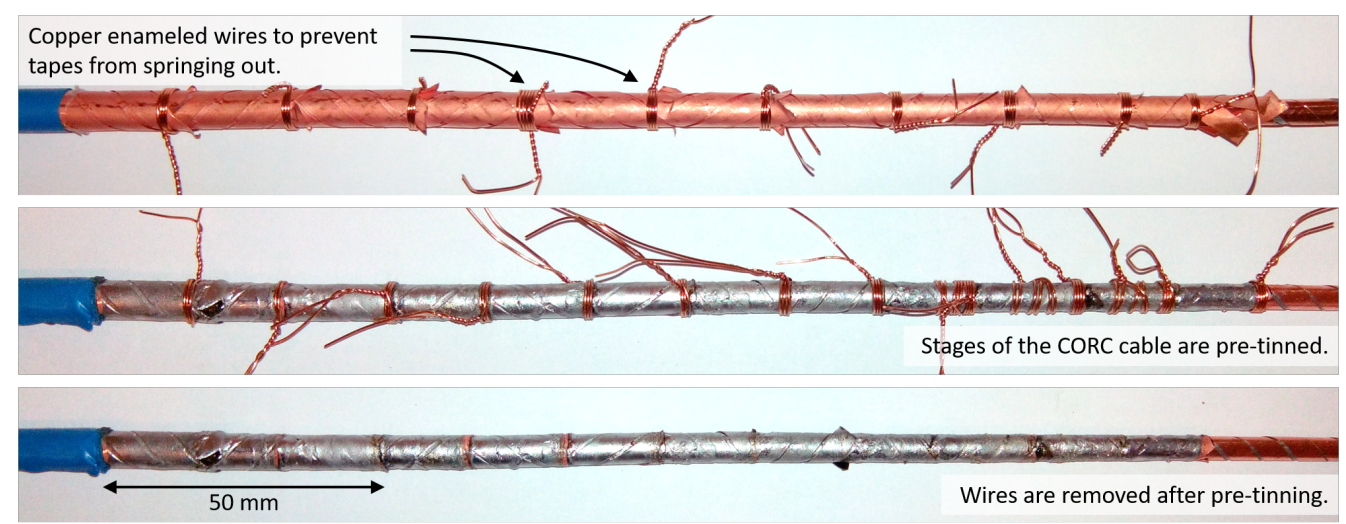

Figure 3.27: Top: the staged CORC cable end with copper enameled wires to prevent the tapes from springing out. Middle: an additional solder 'bandage' is added to keep the stages together after subsequently removing the copper wires. Bottom: the wires are removed and the CORC cable end can be inserted in a copper terminal.

No twisting is required with cylindrical terminals, as these have a radial symmetry. The cylindrically shaped terminals can be clamped or soldered to other current leads. A picture of a rectangular joint terminal is presented in Figure 3.28 and a staged cylindrical terminal is presented in Figure 3.29.

\section{Solder Filling of the Terminals}

Choosing the right type of solder is an important issue in the joint preparation process. The melting point of the solder has to be lower than $200^{\circ} \mathrm{C}$. Exceeding this temperature can cause degradation to the ReBCO tapes as oxygen may diffuse out of the ceramic layer $[86,87]$. Therefore it is important to monitor the temperatures of the joint terminal and CORC conductor itself during the filling procedure. Before filling, the entire joint terminal and the staged end of the CORC conductor are heated to a temperature just above the melting point of the solder. The bandage of solder around the prepared stages melts and tapes spring out. Additional solder is added to fill the voids between the tapes and thus to form the electrical connection from terminal to CORC.

Various solder metals can be used for filling and pre-tinning. A common, inexpensive solder with a relatively high electrical conductivity is Sn63Pb37 with a melting temperature of $183^{\circ} \mathrm{C}$. This solder wets both the copper stabilizer on the tapes and the inner surface of the copper terminals well. It also allows a later solder connection between different terminals or conductors with another soldering material that has a lower melting temperature than the one used inside the terminals. Another good solder to use is pure indium. Indium has a high electrical conductivity, however wets slightly less well than $\mathrm{SnPb}$ type solder. Other indium based solders (i.e. In44Sn42Cd14) with even lower melting temperatures can be used as well with similar results. Solder with melting temperatures higher than $200^{\circ} \mathrm{C}$ should be avoided. Also solders that comprise bismuth are 


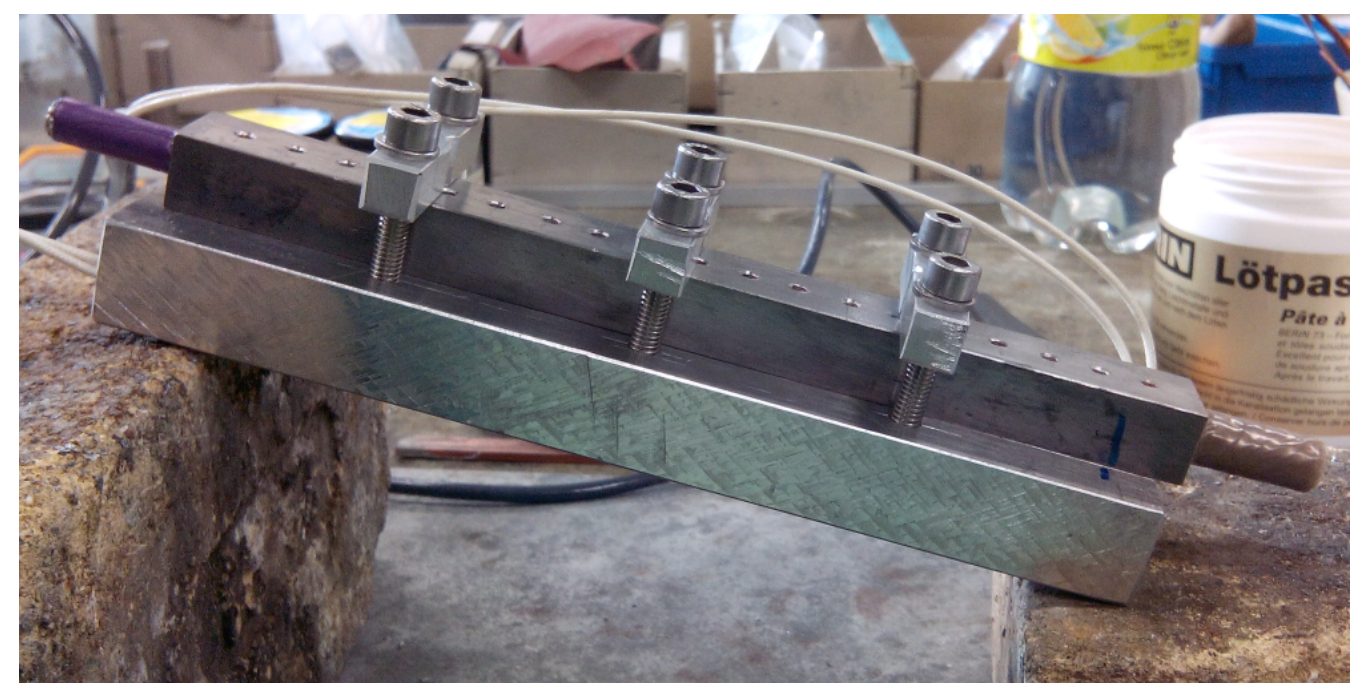

Figure 3.28: Photo of a $200 \mathrm{~mm}$ long rectangular joint terminal that is used to test the solder filling procedure. The terminal is clamped to an aluminium block that embeds two cartridge heaters and a temperature sensor. The filling procedure was tested in horizontal, inclined and vertical positions. The terminal has 19 small holes on its long face for solder filling and to allow gas to escape.

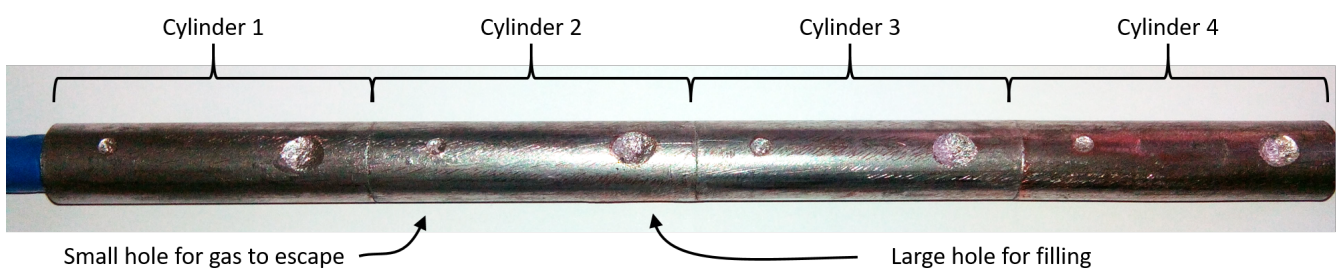

Figure 3.29: Four cylindrical tubes of each $50 \mathrm{~mm}$ long with different bore diameters. The bore diameters match the outer diameter of the CORC stages to which they are attached in order to minimize the amount of solder in the terminals. Each cylinder has two holes; a large one for solder filling and a smaller one for gas to escape. The ends are closed with Teflon tape to prevent the solder from flowing out.

not recommended, since these tend to form micro-cracks when cooled down to cryogenic temperatures. Silver-containing solders, i.e. InAg, are also not recommended, since the silver layer on the ReBCO tapes may dissolve in the solder.

The filling procedure was tested by preparing several prototype joint terminals. One of the first trial units was a cylindrical copper tube terminal used with a CORC cable comprising 38 ReBCO tapes in 12 layers. The cable ends are staged in six different sections and inserted into four copper tubes with different inner diameters, as shown in Figure 3.29. The tubes are filled with Sn63Pb37 while being held horizontally. The tubes has two holes each, one for filling and one for gas venting. The end of the tube terminal is closed with a plug of Teflon tape to prevent solder from leaking out. This method was later used to 
prepare terminals for a $1.8 \mathrm{~m}$ long CORC cable tested at $4.2 \mathrm{~K}$ in an external magnetic field.

A similar test was performed with a rectangular terminal of $200 \mathrm{~mm}$ in length. The terminal has nineteen holes on one of its sides that are used for solder filling and allows for the escape of gas. The bore of this terminal has two different inner diameters; one section has a diameter of $9 \mathrm{~mm}$ and the other a diameter of $7 \mathrm{~mm}$. The end of the prepared CORC cable has 6 sections. The three stages at the end of the CORC cable fit in the section with the thinner bore and the other three fit in the section with the larger bore. The terminal is clamped to an aluminium block fitted with two cartridge heaters and is inclined such that any gas pockets flow towards the holes, as shown in Figure 3.28. The holes are closed by applying Teflon tape over the hole once the solder started flowing out, this process was continued until the entire terminal was filled with solder.

\section{Joint Issues}

Cross-sectional slices of some selected joint terminals were prepared and polished to optically investigate the filling factor of the solder and the amount and location of possible remaining gas pockets. One cylindrical terminal was extracted from a CORC cable after its test and opened for inspection. Two slices of its cross-section are presented in Figure 3.30. The cross-sections clearly show that there are still several gas pockets between tapes. The gas pockets are mainly present in areas with a high tape density which offer less space for the gas to escape. A larger bore allows the tapes to spring out further and facilitates gas escaping the terminal's bore. The cross-sections presented in Figure 3.31 show less gas pockets. These are slices from the rectangular terminal that was filled at an inclined position (Figure 3.28) and this terminal has many holes for the gas to escape. The inclined to fully vertical filling orientation reduces the amount of gas pockets compared to the horizontal filling position and therefore is preferred for better filling results.

\subsubsection{Terminals for CORC Cable-In-Conduit Conductors}

The preparation protocol for joint terminals of CORC Cable-In-Conduit Conductors is a scaling up, but still very similar to terminals of single CORC conductors. All CORC strands are staged using the methods described in Section 3.5.1. Then the strands are bundled and inserted in the copper terminal. Thereafter, the terminal is filled with solder.

A demonstration CORC CICC joint terminal was prepared using dummy CORC strands to validate and practice all preparation steps. The ReBCO tape in the dummy strands are replaced by two layers of $50 \mu \mathrm{m}$ thick copper tape followed by two layers of $50 \mu \mathrm{m}$ thick stainless steel tape. The ends of the strands are staged over a length of $350 \mathrm{~mm}$ into 5 sections of 4 layers each (equivalent to $2 \mathrm{ReBCO}$ layers per section). Thereafter, the strands are bundled around a copper tube. Figure 3.32 shows the strands during the staging steps as well as the cable bundle. The joint demonstrator comprises a square copper casing of 30 by 30 by $400 \mathrm{~mm}^{3}$. The bore in the terminal is $350 \mathrm{~mm}$ deep and has a diameter of $24 \mathrm{~mm}$ to receive the six-around-one cable bundle. The terminal is 

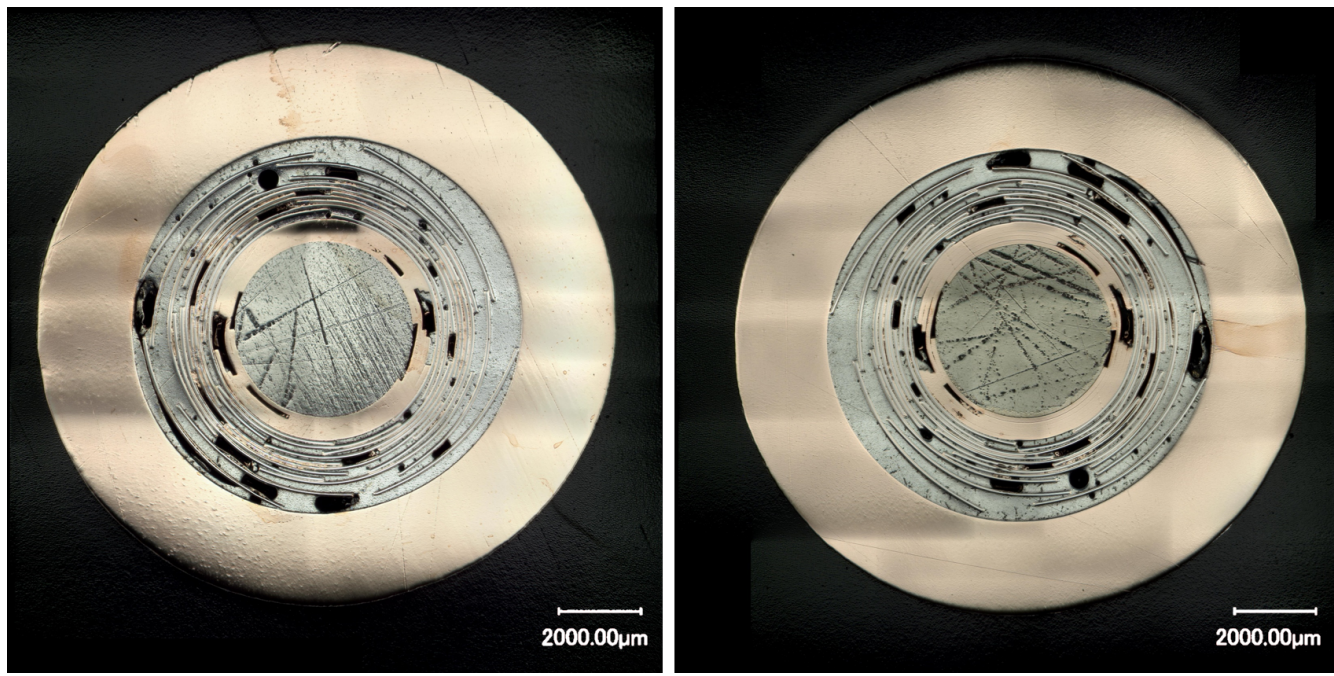

Figure 3.30: Cross-sectional views of two slices from the cylindrical joint terminals shown in Figure 3.29. Several gas pockets are still present in this terminal, which suggest that horizontal filling is not recommended, even with additional holes for gas to escape.
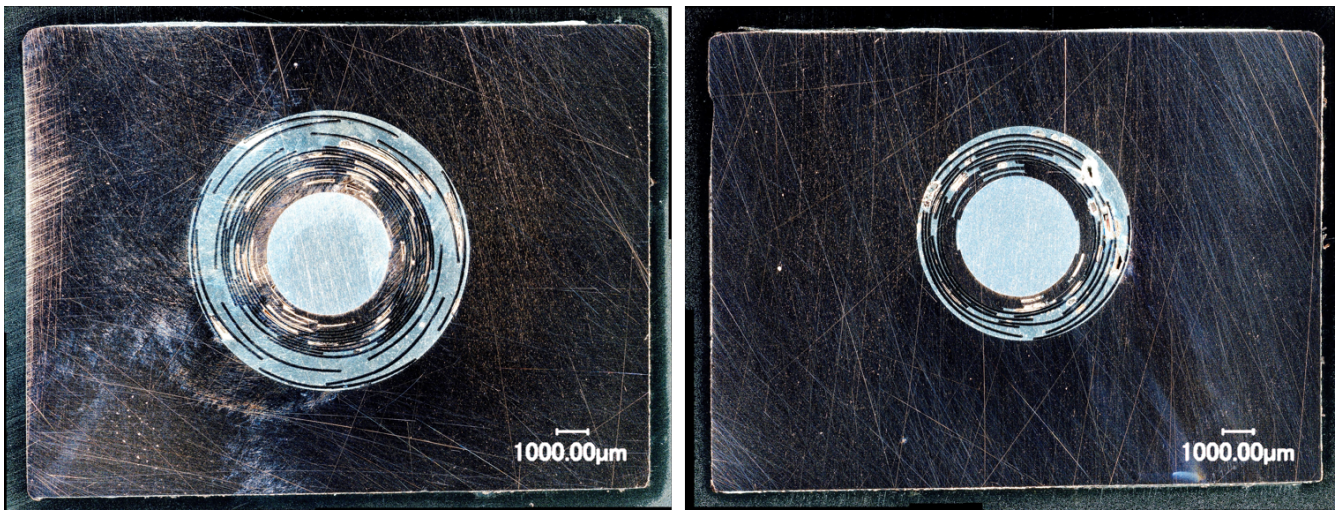

Figure 3.31: Cross-sectional views of two slices from the rectangular joint terminals shown in Figure 3.28. This terminal has two different bore diameters to reduce the solder to copper ratio. The tapes of the outer layers spring out towards the terminal and solder flows between tapes. The photos show an adequate solder filling, yet some gas pockets still remain.

filled in a vertical position with eutectic Sn63Pb37 solder using a non-corrosive soldering flux. The entire copper terminal is heated using cartridge heaters, which are placed in an aluminum block clamped to the terminal. The temperature is measured and regulated at about $200 \pm 5^{\circ} \mathrm{C}$. As discussed in Section 3.5.2, long periods of time at this temperature does not cause any degradation of the ReBCO tape [86]. The temperature of the terminal was kept stable at $200^{\circ} \mathrm{C}$ for 5 minutes once the terminal was completely filled with solder, while vibrating it slightly to loosen and remove excess gas still trapped inside. The molten 
solder shrinks during cool down and additional solder is added until the solder in the casing solidified.

Several cross-sections were cut from this demonstrator joint terminal to inspect the filling with solder material, shown in Figure 3.33. The most uniform solder filling is found near the bottom of the joint, where the staged CORC strands are at their thinnest and gas pockets can flow upwards and escape. The CORC strands are thicker near the exit of the terminal's bore, resulting in more gas pockets being stuck inside the terminal. Nevertheless, the amount of remaining gas pockets is marginal, hardly affecting the performance of the joint terminal.

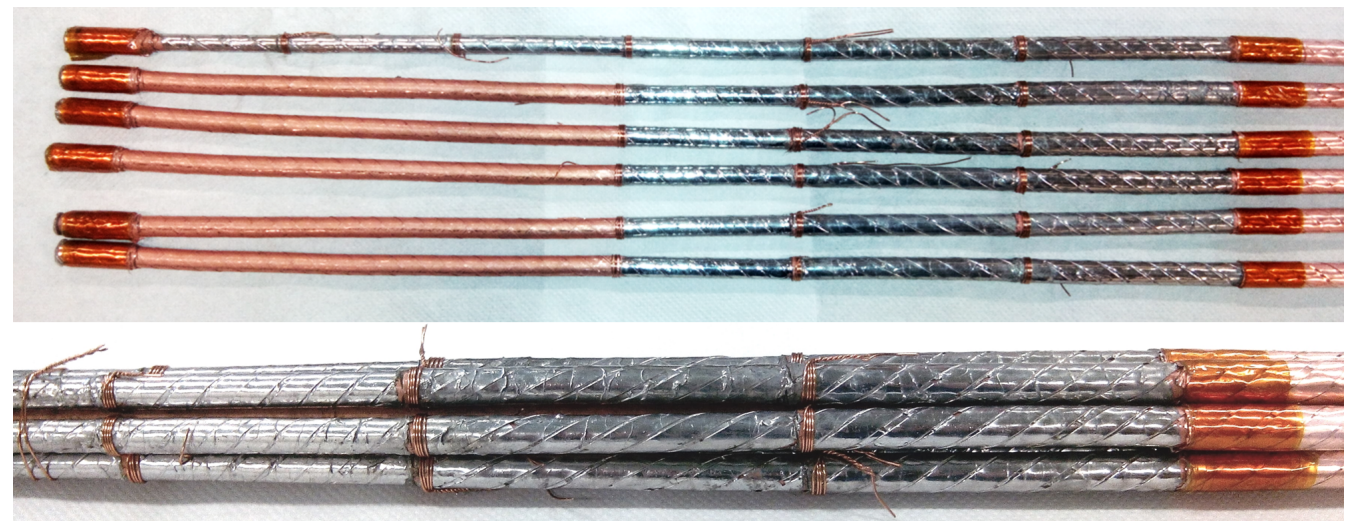

Figure 3.32: Staged (top) and bundled (bottom) six CORC dummy strands for the demonstrator CORC Cable-In-Conduit Conductor and its joint terminal. The top picture shows the six CORC strands during preparation of the stages of the ReBCO layers over a length of $350 \mathrm{~mm}$. The bottom picture shows the joint section of the bundled six-around-one cable.

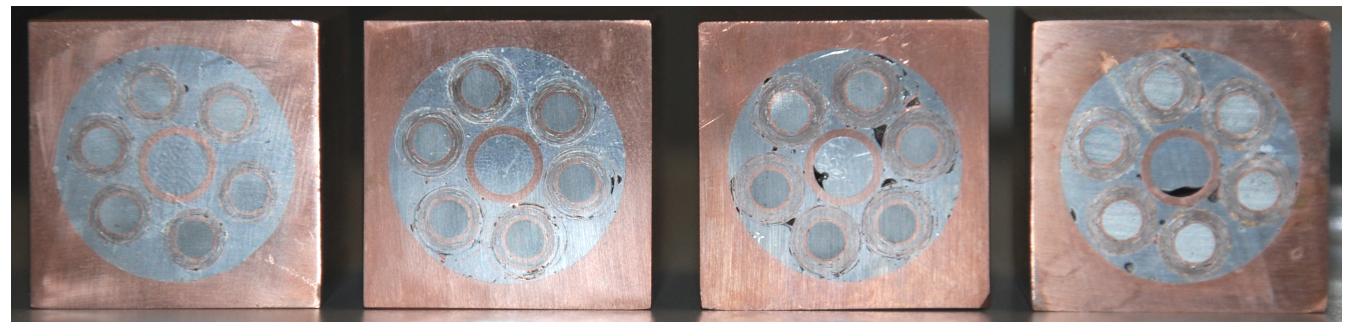

Figure 3.33: Cross-sections prepared from the demonstrator joint terminal, each 30 by $30 \mathrm{~mm}^{2}$ in size. The cuts are from the deepest point in the joint terminal (left) to near the exit of the terminal (right). A few gas pockets are still present in the terminal, mainly around the central tube, but are expected to have negligible effect on the contact resistance of the strands. 


\subsection{Experimental Realization of CORC Joint Terminals}

The CORC samples tested during this work all comprised joint terminals of the novel design. In total one CORC Cable, four CORC wires and three CORC Cable-In-Conduit Conductors were tested, which amounts to a total of 16 joint terminals. The experimental performance of the joint terminals is described in Chapters 4 and 5 of this thesis, together with the performance of the CORC conductors themselves.

\subsection{Conclusion}

Electrical connections to the CORC conductor are a key factor in current sharing between the conductor's different tapes and layers, especially during quasi-DC operation. A homogeneous current distribution between all ReBCO tapes avoids a premature superconducting to normal transition and ensures high stability. As a second requirement, a low joint resistance is needed to limit joule heating and thereby also limiting cooling requirements and costs. However, a too low joint resistance may have negative effects on the current distribution in the CORC conductor, since the effect of small defects in the joint then becomes relatively more pronounced and can cause inhomogeneity in the current distribution.

A novel joint design was developed for the CORC conductor, aimed at further improving the current sharing and reducing the overall joint resistance compared to previous joint designs. The novel design introduces staging of the ReBCO layers in the CORC conductor over the joint section to form a staircase-like geometry, which allows current to flow more directly to the inner ReBCO layers of the conductor. A reduction in contact resistance of $80 \%$ at $80 \%$ of the cable's critical current is realized and close to perfect current distribution among layers by optimizing the stage lengths.

Several demonstration and prototype joint terminals were prepared for both single CORC conductors and CORC Cable-In-Conduit Conductors, in order to practice and evaluate all preparation steps involved. All steps proved feasible and successful in achieving the desired staging and solder filling. Still, inspection of some of the joint terminals showed that a few gas pockets remained within the solder filling. The gas pockets are relatively small and do not affect the performance of the joint terminal significantly. Inclined filling results in less gas pockets than horizontal filling. More experience in handling and filling will further improve preparation quality and consistency. 


\section{Chapter 4}

\section{CORC Wires and Cables}

Conductor On Round Core (CORC) conductors and their production technique were new and in the early stage of development at the start of this work. A better understanding on the performance of the CORC conductor, current sharing between layers, current distribution between individual ReBCO coated conductors, CORC bending/handling and all details appertained to the production of the CORC conductor are required. In this chapter, methods for predicting the performance of CORC conductors are discussed, experiments on CORC cables and wires are described and their results are reported.

\subsection{Introduction}

CORC conductors comprise many ReBCO tapes in many layers and come in the shape of a CORC cable with a diameter ranging from 5 to $8 \mathrm{~mm}$ or in the shape of a CORC wire, which are thinner with a diameter ranging from 2.5 to $4 \mathrm{~mm}$. The performance of a CORC conductor is not simply a summation of the performance of the straight ReBCO coated conductors. Therefore, techniques are developed for predicting CORC conductor performance as function of magnetic field and temperature on the basis of single tape performance.

In this chapter two experiments on a CORC cable and several wires are presented. The characterized CORC cable is designed for use as strand in the first-in-the-world CORC Cable-In-Conduit Conductor and the state-of-the-art CORC wires aim for direct application in high field magnets. The experimental results are compared to predicted values to validate and fine-tune the scaling methods. Several CORC wires were prepared by Advanced Conductor Technologies and tested at the University of Twente in a common research effort of ACT, University of Twente and CERN. The CORC wires were tested to demonstrate their mechanical flexibility and high current carrying performance and to provide valuable feedback for further optimization of production and handling techniques. A ReBCO CORC racetrack dipole magnet is under development at CERN to demonstrate the high performance of the first generation of CORC wires. 


\subsection{Predicting the Performance of CORC}

Accurately predicting the performance of CORC conductors is important to avoid failures when applying this expensive conductor. Understanding ReBCO tape, the primary component of the CORC conductor, is key in predicting CORC conductor performance. The performance of ReBCO coated conductors is highly dependent on magnetic field, magnetic field angle, temperature and geometrical shape. This section sets out scaling relations that are fundamental in predicting CORC conductor performance and expands on the fitting formulas applied to quantify and extrapolate the performance of measured CORC conductors.

\subsubsection{Geometrical Scaling}

In Section 2.3.1, it was shown that per meter of conductor between $1.3 \mathrm{~m}$ (CORC cables) and $1.6 \mathrm{~m}$ (CORC wires) of ReBCO tape is required to form their helical shape. The critical current of the CORC conductor is thus per definition lower than the sum of critical currents of the ReBCO tapes. The performance of the ReBCO tapes are described by their critical current and n-value following the power-law (Equation 2.1). The electric field over a spiraled tape is given by equation 4.1, which includes both the power law and the effective reduction in length due to the helical shape of the tape:

$$
E(I)=\frac{\sqrt{\left(2 \pi r_{a}\right)^{2}+p_{a}^{2}}}{p_{a}} U_{0}\left(\frac{I}{I_{c t}}\right)^{n}
$$

Here $E$ is the electrical field of the spiraled tape, $r$ and $p$ are the radius of the spiral and its pitch length, respectively, and $I_{c t}$ is the tape's critical current. The critical current of the CORC conductor can be calculated by solving equation 4.2.

$$
U_{0}\left(\frac{I}{I_{c c}}\right)^{n}=\frac{\sqrt{\left(2 \pi r_{a}\right)^{2}+p_{a}^{2}}}{p_{a}} U_{0}\left(\frac{I}{I_{c t}}\right)^{n}
$$

By assuming the n-value does not change due to spiraling the tapes, the following ratio between spiraled and straight tape critical currents is found:

$$
\frac{I_{s p}}{I_{s t}}=\frac{1}{\left(\frac{\sqrt{\left(2 \pi r_{a}\right)^{2}+p_{a}^{2}}}{p_{a}}\right)^{\frac{1}{n}}} .
$$

Effectively, a critical current reduction of approximately $4 \%$ is found for thick CORC cables with long twist pitches and large winding radii and about $6 \%$ for the thin CORC wires compared to the summation of all critical currents of each straight ReBCO tape, as presented in Table 4.1. Here, uniform current sharing among all tapes is assumed and additional electric fields due to cross-effects ignored, such as the effect of self-field. 
Table 4.1: Critical current retention coefficient $c_{g}$ of 2, 3 and $4 \mathrm{~mm}$ wide tapes wound into a CORC conductor due to geometric scaling.

\begin{tabular}{lccc}
\hline \hline & CORC Cables (SCS2xxx) & CORC Wires (SCS3xxx) & CORC Wires (SCS4xxx) \\
\hline $\mathrm{I}_{c}$ retention & 0.94 & 0.95 & 0.96 \\
\hline \hline
\end{tabular}

Added stabilizer material forms a parallel path for the current. The core of the CORC conductor is usually metallic, prepared from a material with high electrical conductivity such as copper or aluminium. An electric field over the conductor causes current sharing between ReBCO tapes and stabilizer material. The amount of current in the stabilizer depends on its resistance and has a small effect on the conductor's critical current and $\mathrm{n}$-value. The current in the stabilizer at $\mathrm{I}_{c}$ is given by:

$$
I_{\text {stab }}(T)=U / R_{\text {stab }}(T) .
$$

At $77 \mathrm{~K}$ the resistivity of the common stabilizer materials aluminium and copper is substantial compared to their resistivity at $4.2 \mathrm{~K}$ and huge compared to the effective resistivity of the superconductor at its critical current. The expected current at $77 \mathrm{~K}$ in the stabilizer of a typical CORC conductor at an electric field of $100 \mu \mathrm{V} / \mathrm{m}$ is about a few amperes. At $4.2 \mathrm{~K}$ the current in the stabilizer is larger and some dozens of amperes. The current in the tapes of the CORC conductor is in both cases likely several orders of magnitude higher. Therefore, the current in the stabilizer can be neglected. The critical current of the CORC conductor is given by:

$$
I_{c}=I_{c c}+I_{s t a b} \approx I_{c c}
$$

The stabilizer does not have a substantial impact on the critical current of a CORC conductor, therefore it can be ignored when predicting the CORC conductor's stationary performance.

\subsubsection{Scaling with Temperature and Magnetic Field}

The performance of ReBCO tapes strongly depends on temperature and magnetic field. Tape suppliers commonly test entire tape-batches only at $77 \mathrm{~K}$ in self-field. Tape performance at a lower temperature than $77 \mathrm{~K}$ and in magnetic field can be estimated by using generic scaling laws or better, by characterizing short tape sections from the batch to determine the tape's lift factor as function of temperature and magnetic field. Tapes in a CORC conductor follow the same temperature and similar magnetic field scaling laws as single tapes. An equation that provides a good estimation of the performance of ReBCO tape from several tape manufacturers as function of temperature and magnetic field is provided by Senatore et al. in [88]:

$$
J_{c}(T, B)=J_{c}(T=0, B=0) e^{-T / T^{*}} B^{-\alpha} .
$$


Here $T^{*}$ and $\alpha$ are scaling parameters for temperature and magnetic field. Values of the scaling parameters for SuperPower tapes are provided in Figure 4.1 [88] and are able to fit the tape critical current density in the temperature range of 4.2 to $50 \mathrm{~K}$ with an accuracy of $2 \%$. There is still quite some variance in lift factors between tapes from different manufacturers and even between tape batches from a single manufacturer depending on tape optimization for $4.2 \mathrm{~K}$ or for $77 \mathrm{~K}$, high-field use. Therefore, the equation can only be used to provide a first order estimate of tape performance.

A better method to determine the tape lift factor is to measure the critical current of a tape sample of the batch in magnetic field at 4 and $77 \mathrm{~K}$. Lift factors for SuperPower SCS4050 tape without Advanced Pinning were measured by Braccini et al. [53] and are shown in Figure 4.2. Advanced pinning of SuperPower tape increases its performance at $4 \mathrm{~K}$ in the high-field regime as demonstrate in literature by Tsuchiya et al. [51] and thereby increasing the tape lift factor $\mathrm{I}_{c}(4.2 \mathrm{~K}, 10 \mathrm{~T}) / \mathrm{I}_{c}(77 \mathrm{~K}$, self-field) from 2 to approximately 2.5 to 3 . The lift factor 2.75 is used here to predict CORC conductor performance at $4.2 \mathrm{~K}$ and $10 \mathrm{~T}$ for CORC conductors with tapes not tested at $4.2 \mathrm{~K}$ and in magnetic field.

The angle between magnetic field vector and tape surface is not constant per tape since the tapes are curved around the conductor's core. Cross-sectional views of two CORC conductor examples are presented in Figure 4.3. The figure shows the curvature of each tape in the CORC conductor. A single tape endures both parallel and perpendicular magnetic field when the conductor is exposed to an external magnetic field, which causes a non-uniform critical current distribution over the tapes cross-section. The critical current distribution over the tape's width and the resulting effective critical current is calculated by using Equation 2.2. Spiraled SuperPower tapes without Advanced Pinning technology commonly have an effective critical current of $30 \%$ to $40 \%$ of $\mathrm{I}_{c}(4.2 \mathrm{~K}, 10 \mathrm{~T})(\mathrm{B} / / \mathrm{a})$ in the curved shape [57]. More recent tapes with 7.5\% Zirconium doping have effective critical currents between $45 \%$ and $60 \%$ of $\mathrm{I}_{c}(4.2 \mathrm{~K}, 10 \mathrm{~T})(\mathrm{B} / / \mathrm{a})$ [56] and the latest tapes with $15 \%$ Zirconium doping show an effective critical current of $60 \%$ and higher compared to the $\mathrm{I}_{c}(4.2 \mathrm{~K}, 10 \mathrm{~T})(\mathrm{B} / / \mathrm{a})$. Engineering values for the normalized effective critical current are presented in Table 4.2 for SCS4050 and SCS4050-AP tapes.

Table 4.2: Effective normalized critical current coefficient $c_{b}$ of ReBCO tape curved around the CORC conductor's core for SCS4050 [57] and SCS4050-AP tapes.

\begin{tabular}{lcc}
\hline \hline B (T) & SCS4050 & SCS4050-AP \\
\hline 0 & 1 & 1 \\
2 & 0.54 & - \\
4 & 0.44 & - \\
6 & 0.39 & - \\
8 & 0.35 & - \\
10 & 0.33 & $0.45-0.6[56]$ \\
12 & 0.31 & $0.51[89]$ \\
14 & 0.29 & - \\
16 & 0.28 & - \\
18 & 0.27 & - \\
20 & 0.26 & - \\
\hline \hline
\end{tabular}



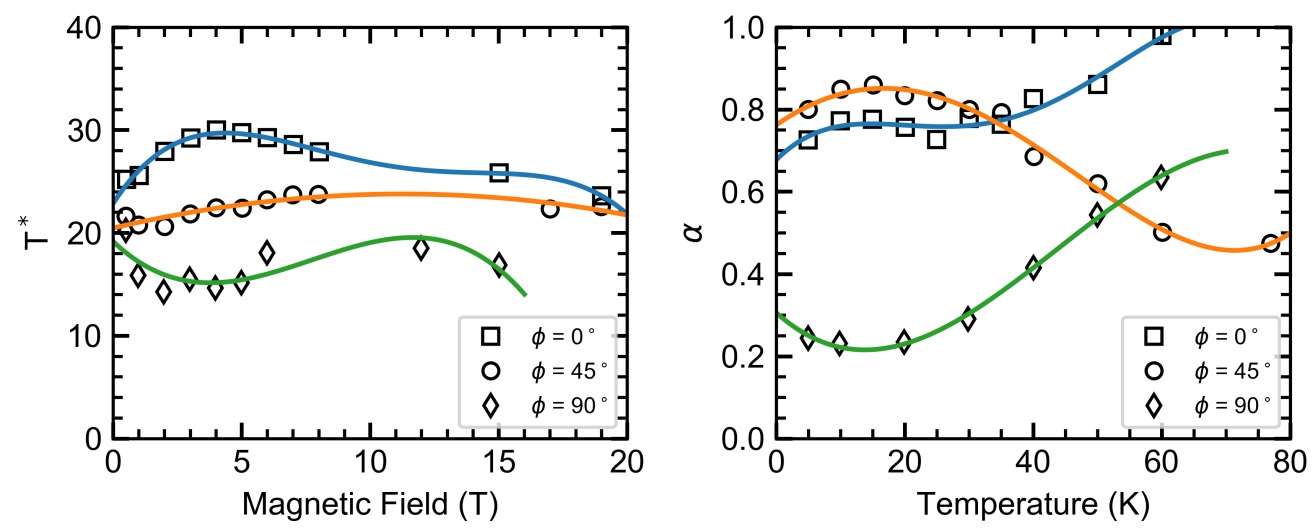

Figure 4.1: Scaling parameters $T^{*}$ and $\alpha$ as function of magnetic field and temperature for SuperPower tapes for use in Equation 4.6, reported by C. Senatore et al. [88].

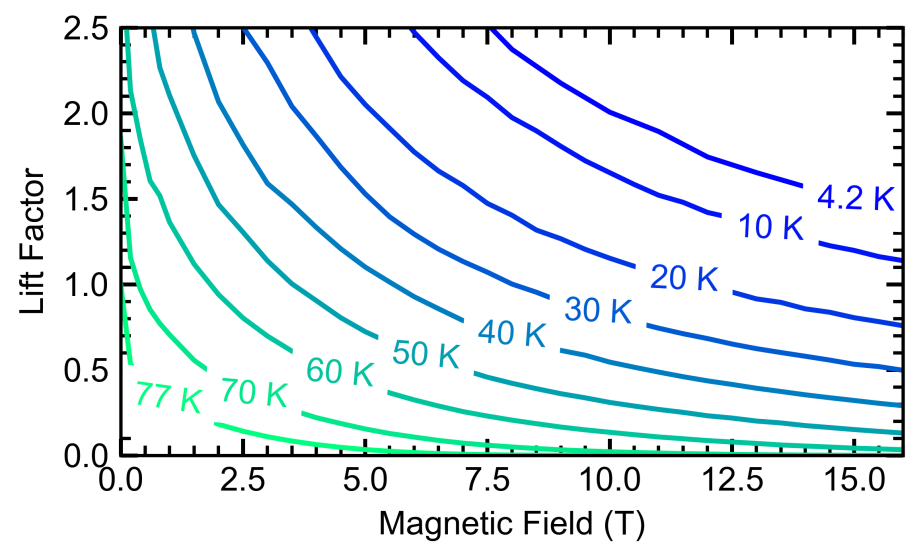

Figure 4.2: Measured lift factors as function of perpendicular magnetic field for various temperatures for SuperPower Tape without Advanced Pinning (AP) measured by Braccini et al. [53].

The total critical current of a CORC conductor can be predicted by applying the following equation using the tape lift factor and the values from Tables 4.1 and 4.2:

$$
I_{c}=n I_{c t}(77 \mathrm{~K}, \text { Self-Field }) L(\mathrm{~T}, \mathrm{~B}) c_{g} c_{b} .
$$

Here $n$ is the number of ReBCO tapes, $L$ is the tape lift factor, $\mathrm{c}_{g}$ is the geometric $\mathrm{I}_{c}$ retention coefficient (Table 4.1) and $c_{b}$ is the magnetic field angle dependent $\mathrm{I}_{c}$ retention coefficient (Table 4.2). 


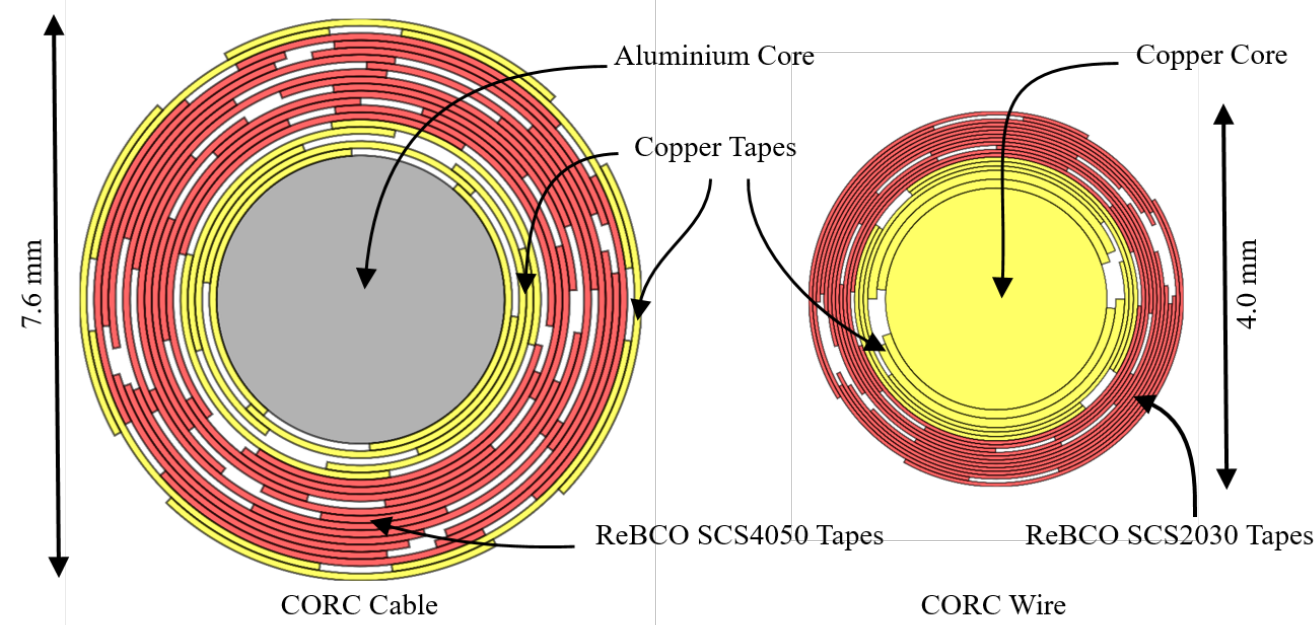

Figure 4.3: Schematic layout of the cross-sections of one thick CORC cable and a thin CORC wire. The layouts are based one two CORC conductors measured at CERN and the University of Twente. The CORC cable comprises standard SuperPower SCS4050 tapes and the CORC wire comprises state-of-the-art 2 mm wide SCS2030 tapes.

\subsubsection{Fitting Equation for ReBCO Based Conductors}

All measured conductor electrical fields as function of current are fit with the power-law to determine the conductor's critical current and n-value:

$$
U=U_{0}\left(\frac{I}{I_{c}}\right)^{n}+I R+c
$$

The power-law has a non-linear part that includes the superconducting transition. The electric field criterion used to determine the critical current in $100 \mu \mathrm{V} / \mathrm{m}$. The linear part in the power-law function provides the value of a measured constant resistance. A constant resistance is commonly present when a splice is included between voltage taps or when there is a significant defect in the conductor that causes current to redistribute. The last part of the power-law is a constant that removes the offset from the measured data.

The measured critical current as function of magnetic field are fitted and extrapolated with the following equation to assess the conductor's performance as function of magnetic field:

$$
I_{c}(B)=a\left(1+\frac{B}{b}\right)^{c},
$$


where $a, b$ and $c$ are fitting parameters. The fit is tested using measured critical current data of SuperPower tape [53] as function of magnetic field for various temperatures as presented in Figure 4.4. The data ranges from 0 to $16 \mathrm{~T}$ and the fit is based on the data in the range of 5 to $10 \mathrm{~T}$, which is a common measurement range in superconductor test facilities. In the lower magnetic field range of 0 to $2.5 \mathrm{~T}$ some inaccuracies are observed, especially using measurement data taken at higher temperature. The fit is able to accurately extrapolate the critical current to higher magnetic field over the entire measurement range of 10 to $16 \mathrm{~T}$ and can therefore be used to predict, with reasonable precision, the conductor performance up to $20 \mathrm{~T}$, the maximum considered.

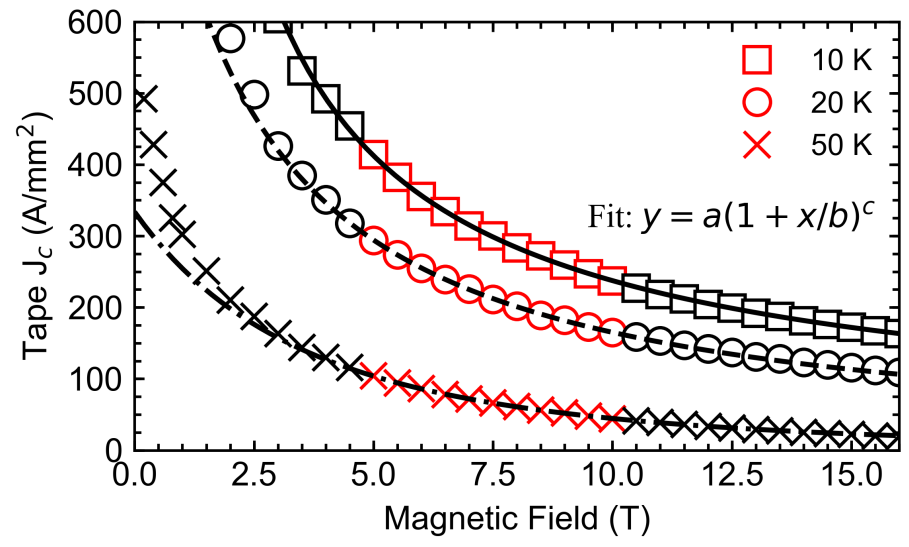

Figure 4.4: Critical current density of SuperPower ReBCO tapes for a temperature of 10, 20 and $50 \mathrm{~K}$ from Braccini et al. [53] fit with Equation 4.9 using only the 5 to $10 \mathrm{~T}$ data (marked in red). The fit function is able to extrapolate the data accurately to magnetic fields in the measured data range of 10 to $16 \mathrm{~T}$.

\subsection{Performance of a Single CORC Cable}

The CORC cable, often with a diameter larger than $6 \mathrm{~mm}$, can be used stand-alone for power transport applications or as a strand in a multi-strand conductor. This type of CORC conductors have a thick core that ensures good thermal and electrical stability, but slightly lack in current density. In 2014, CERN has acquired a $12 \mathrm{~m}$ long CORC cable, record length at that time, for preparation and testing of a first six-around-one CORC Cable-In-Conduit Conductor, including testing of joint terminals following a novel design (see Section 3.3). Quantification of the in-field performance and the limitations of the single CORC cable/strand is important to accurately predict the performance of the larger cable-in-conduit conductors. Therefore part of the development program was the characterization of the CORC cable at 1.9 and $4.2 \mathrm{~K}$ in an external magnetic field. The main goal of this test is to map the conductor's critical current as function of magnetic field and to compare to predicted values. The joints used for this CORC cable are of the novel joint design and are one of the first joints of this type ever tested at cold and in 
Table 4.3: Properties of the first $12 \mathrm{~m}$ long CORC cable procured by CERN.

\begin{tabular}{lcc}
\hline \hline Property & Value & Unit \\
\hline ReBCO tape & SuperPower SCS4050 & - \\
Cu stabilizer (top/bottom) & $20 / 20$ & $\mu \mathrm{m}$ \\
Number of ReBCO tapes & 38 & - \\
Number of copper tapes & 18 & - \\
Number of layers & 12 & - \\
Outer diameter (including sleeve) & 7.8 & $\mathrm{~mm}$ \\
Outer diameter (without sleeve) & 7.6 & $\mathrm{~mm}$ \\
Core material & $\mathrm{Al} 1350$ & - \\
Core diameter & 4.0 & $\mathrm{~mm}$ \\
Core diameter including Cu tapes & 5.2 & $\mathrm{~mm}$ \\
\hline \hline
\end{tabular}

magnetic field. The test provides important feedback used to improve and optimize CORC joint design.

\subsubsection{CORC Sample and Test Setup}

The CORC sample is $1.8 \mathrm{~m}$ long and taken from a $12 \mathrm{~m}$ long CORC cable. The cable has 38 ReBCO SuperPower SCS4050 tapes wound in 12 layers around an aluminum core. The outer three ReBCO layers comprise 4 tapes each, the next eight layers comprise 3 tapes each and the inner most layer has 2 tapes. Two extra layers of copper tape and a polyethylene sleeve are added for mechanical protection. The tapes are taken from different batches with critical currents ranging from 100 to $140 \mathrm{~A}$ at $77 \mathrm{~K}$. Table 4.3 provides additional geometrical properties of the CORC sample.

The test is performed in the FRESCA cable test facility at CERN [90]. FRESCA features a $10 \mathrm{~T}$ dipole magnet with a homogeneous magnetic field region extending over $600 \mathrm{~mm}$. The bore tube has an inner diameter of $71 \mathrm{~mm}$ in which a sample can be inserted for a test at $1.9 \mathrm{~K}$ and $4.2 \mathrm{~K}$ with stationary currents up to $32 \mathrm{kA}$. The sample holder is of the hairpin type and has superconducting 'go' and 'return' legs for current to pass over the high-field region, as shown in Figure 4.5. The CORC cable is used as the go-leg and a stack of three NbTi LHC type Rutherford cables shape the return-leg. The CORC cable is clamped on the sample holder using several brass brackets positioned about every 150 $\mathrm{mm}$.

The sample holder is inserted vertically in the bore-tube of the magnet. A stainless steel support structure locks the sample in the bore-tube to prevent sample rotation. The hairpin sample is positioned in the background field such to have the forces on the legs directed towards each other.

The electric field over the sample is measured with several voltage taps spread over the conductor length. The high-field region of the FRESCA magnet spans $600 \mathrm{~mm}$ and is located in the center of the sample. The voltage taps in the high-field region are placed $270 \mathrm{~mm}$ apart and include multiple twist pitches of the outer tapes. Voltage taps are 
attached to every ReBCO tape in the outer layer of the sample, which allows monitoring of single tape electric field. The voltage taps are visible in Figure 4.6.

The joints are positioned outside the peak magnetic field region and comprise a splice between NbTi current leads to a copper block and a splice between the copper block to the CORC joint terminal. The CORC joint terminals within the joints are cylindrically shaped and of the novel joint design. Voltage taps are positioned on the copper block and on the CORC cable approximately $50 \mathrm{~mm}$ from the joint. The CORC joint terminals are soldered into rectangular copper joint blocks using In44Sn42Cd14 solder with a melting temperature of $93^{\circ} \mathrm{C}$. NbTi Rutherford cables are soldered in a groove on the copper joint blocks and are routed back to the power supply connectors.

Current distribution in the outer layers of the CORC cable was monitored with voltage taps on the individual tapes. The copper tapes wound around the outer ReBCO layer, which provided mechanical protection, are removed locally to make place for the voltage taps. Voltage taps are soldered to each ReBCO tape in the outer layer. Each tape has 4 voltage taps; one on the 'bottom' side of the sample, one on the 'top' side of the sample and two across the high field region.

The joint terminals are of the novel design (Section 3.3). The ends of the CORC cable were staged to 12 sections; one for each layer. The casing of the terminal comprises three sections of OFHC copper pre-tinned cylinders for a total length of $200 \mathrm{~mm}$ per terminal. The CORC sample is inserted vertically in the measurement station, therefore one joint is labeled 'top' joint and the other 'bottom' joint. The sample is connected to the current leads of the measurement station with LHC Rutherford type NbTi conductors. The NbTi Rutherford conductors are connected via a shaking-hands joint to the top joint terminal of the CORC sample and a praying hands joint at the bottom joint terminal of the sample. The predicted resistance of the CORC joint terminals, without the splice to the NbTi leads, is $15 \mathrm{n} \Omega$ calculated using the numerical model described in Section 3.3.

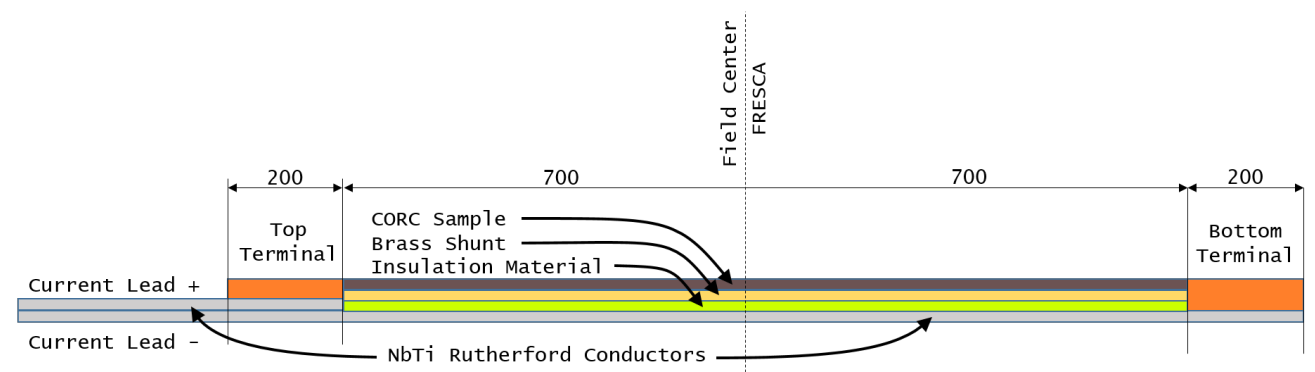

Figure 4.5: Overview of the sample holder used to test the straight CORC cable in the FRESCA cable test facility. The sample length was $1.8 \mathrm{~m}$, which included the joint terminals of $200 \mathrm{~mm}$ each. Current was injected at the 'top' joint terminal and extracted at the 'bottom' joint terminal. NbTi Rutherford conductors connect both terminals to the current leads of the measurement station. 


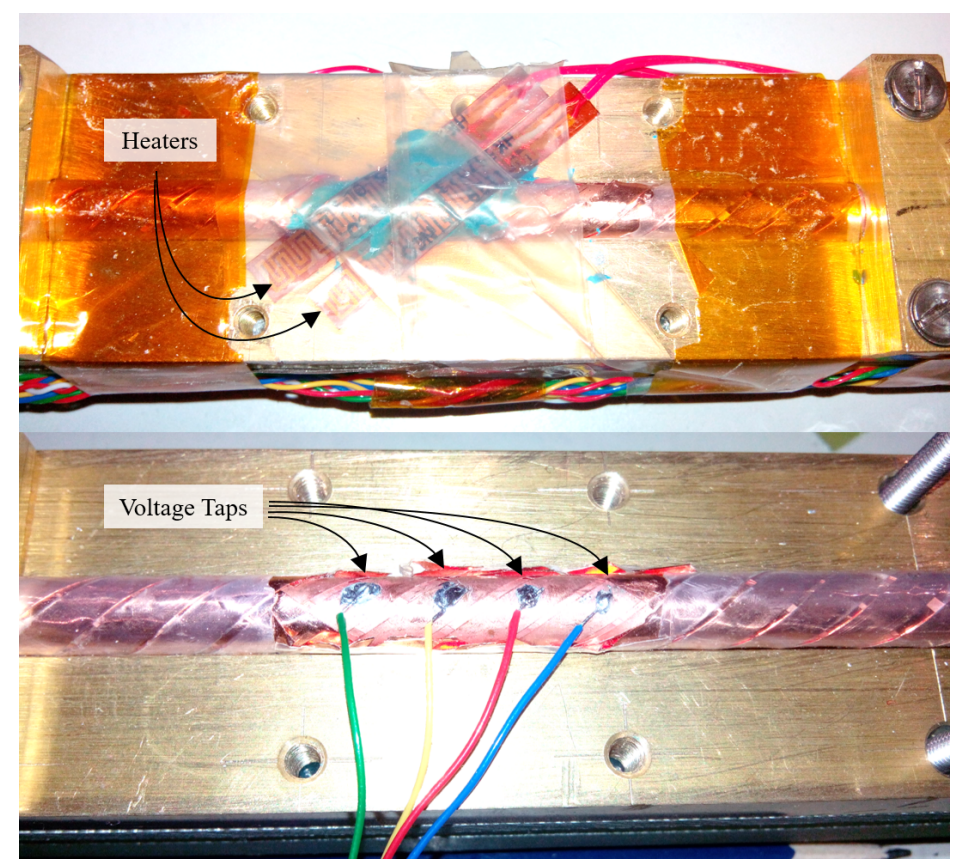

Figure 4.6: Top: Two Minco heaters attached to two single ReBCO tapes located in the outer layer of the CORC cable. Bottom: Voltage taps soldered to each ReBCO tape in the outer layer of the CORC cable to monitor the current distribution in the outer layers.

The quench behavior (and survival rate) of CORC conductors has not been widely explored at the time of this measurement and thus investigated. Quenches are initiated by pushing current over the conductor's critical current or by applying a heat pulse to the tapes in the outer layer using heaters. Two Minco heaters are attached to the sample; one on each of two single adjacent tapes as presented in Figure 4.6. Quenches are initiated by firing one or both heaters at the same time. Voltage over the outer layers during a quench is monitored with the voltage taps on each tape of the outer layer. A brass shunt is present taking over part of the current in the case of a quench in order to avoid damaging the, rather precious, sample. The resistance of the shunt is such that over $98 \%$ of the current flows through the CORC sample up to its critical current. Consequently, voltage measurements can still be performed accurately on the CORC cable. The cable temperature is calculated to reach $300 \mathrm{~K}$ in 0.4 second at a stationary current of $10 \mathrm{kA}$ under adiabatic conditions if the quench is not detected in a timely fashion and if there would be no shunt present.

\subsubsection{Performance of the CORC Cable and its ReBCO Tapes}

The voltage taps on the outer tapes show a clear transition from superconductor to normal state for each of the tapes at the conductor's critical current. There is a small variance in 
the measured conductor critical current for each of the four outer tapes, which indicates that the current is not optimally distributed, as shown in Figure 4.7. Tapes 1 and 3, respectively, transition at a lower current compared to tapes 2 and 4. Each of the tapes in the outer layer are from the same tape production batch and no difference in performance is expected. Therefore, it is likely that the non-uniform current distribution originates in the joint terminals.

The critical current of the CORC cable is determined by fitting the power-law function, with the critical current criterion of $1 \mu \mathrm{V} / \mathrm{cm}$, to the electric field of the two least performing outer tapes. The measured critical current and corresponding current density is presented in Figure 4.7 for $1.9 \mathrm{~K}$ and $4.2 \mathrm{~K}$. The graph includes the self-field correction and shows the expected $\mathrm{I}_{c}$, calculated with Equation 4.7, as reference. The measured $\mathrm{I}_{c}$ is conform expectations in a background magnetic field of $7 \mathrm{~T}$ and higher and the $\mathrm{I}_{c}$ is even larger than expectation in lower magnetic field. The measured n-value is $13 \pm 1$, which is quite a reduction in $n$-value compared to a characteristic n-value of 40 to 50 for a single ReBCO tape at $4.2 \mathrm{~K}$. Early current sharing between tapes in the CORC cable can reduce the conductor's n-value drastically. Commonly, such behavior is observed when current is not distributed uniformly among strands in a multi-strand conductor. A critical current of $8.2 \mathrm{kA}$ is found in a background magnetic field of $9.6 \mathrm{~T}$, which can be extrapolated to a benchmark critical current at $4.2 \mathrm{~K}$ and $10 \mathrm{~T}$ of $7.9 \mathrm{kA}$. The corresponding engineering current density is $174 \mathrm{~A} / \mathrm{mm}^{2}$ at $10 \mathrm{~T} / 4.2 \mathrm{~K}$. Performance is increased by about $5 \%$ by reducing the temperature to $1.9 \mathrm{~K}$. The critical current does not depend on the field-angle, since the CORC cable has an axis-symmetrical tape layout.
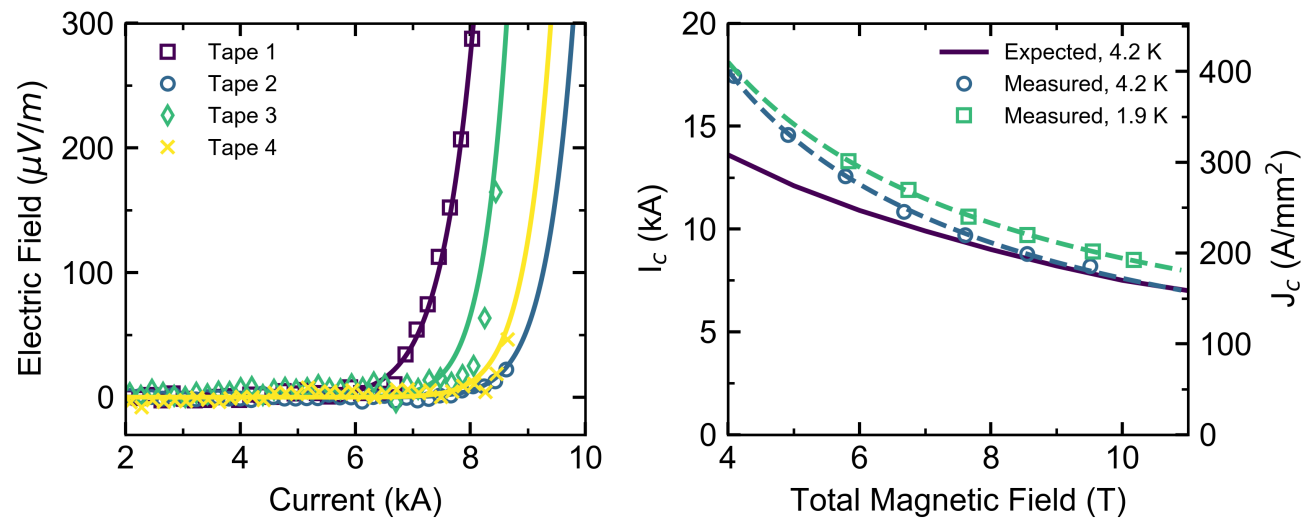

Figure 4.7: Left: electric field over the four ReBCO tapes in the outer layer of the CORC cable during a measurement in $9 \mathrm{~T}$ background magnetic field, $4.2 \mathrm{~K}$ and ramp-rate of $50 \mathrm{~A} / \mathrm{s}$. Right: critical current and the current density as function of the total magnetic field (external + self-field) at 1.9 and $4.2 \mathrm{~K}$ with the expected $I_{c}$ at $4.2 \mathrm{~K}$ as reference. 


\subsubsection{Performance of the Joint Terminals}

The measured resistance of the bottom joint terminal was constant at $30 \pm 1 \mathrm{n} \Omega$ regardless of magnetic field and current. This value is twice the predicted value. A distinct kink in the voltage, measured over the bottom terminal, is observed around $9 \mathrm{kA}$ regardless of magnetic field, which indicates mechanical movement of some part in the sample or its current leads due to the self-field. The slope of the voltage remains unchanged after this kink and therefore the resistance of the bottom joint terminal did not change. The resistance of the top joint terminal varies as function of magnetic field and current. The resistance of the top joint is lower at low current and steadily rises with current up to a certain point. Thereafter, the resistance more or less stabilizes and eventually decreases towards the predicted value of $15 \mathrm{n} \Omega$. The decrease in resistance is possibly related to the Lorentz forces acting on the sample. The CORC joint terminals are pushed towards the joint block in which they were soldered. If the soldering is poor, this additional force may improve contact between CORC terminal and copper joint block and thereby reduce the total joint resistance. The resistance of the top joint terminal in all magnetic fields converges to about $15 \mathrm{n} \Omega$, in accordance with the predicted value.
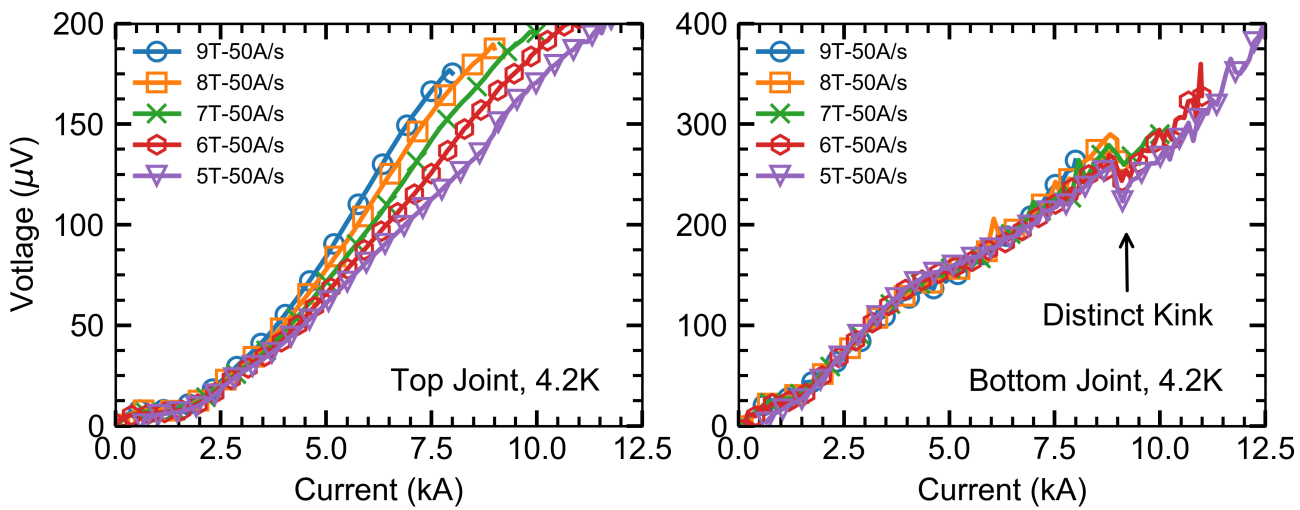

Figure 4.8: Left: Voltage over the top joint terminal as function of current for various magnetic fields (in the high-field region) and a ramp-rate of $50 \mathrm{~A} / \mathrm{s}$. Higher voltages are measured in higher magnetic field. Right: Voltage over the bottom joint terminal as function of current for various magnetic fields and a ramp-rate of $50 \mathrm{~A} / \mathrm{s}$. Voltages over the bottom terminal are in all cases higher than on the top terminal, although they are not dependent on the external magnetic field.

\subsubsection{Quench Behavior and Conductor Survival}

The CORC sample was quenched 11 times; 7 times by using the quench heaters and 4 times by going over the sample's critical current. The quench current (the current where there is thermal runaway in the sample) is probed by increasing the current in small steps. Electric fields with order of magnitude of $1 \mathrm{~V} / \mathrm{m}$ are observed in the conductor before the quench protection is triggered as can be seen in Figure 4.10. The power supply is turned 


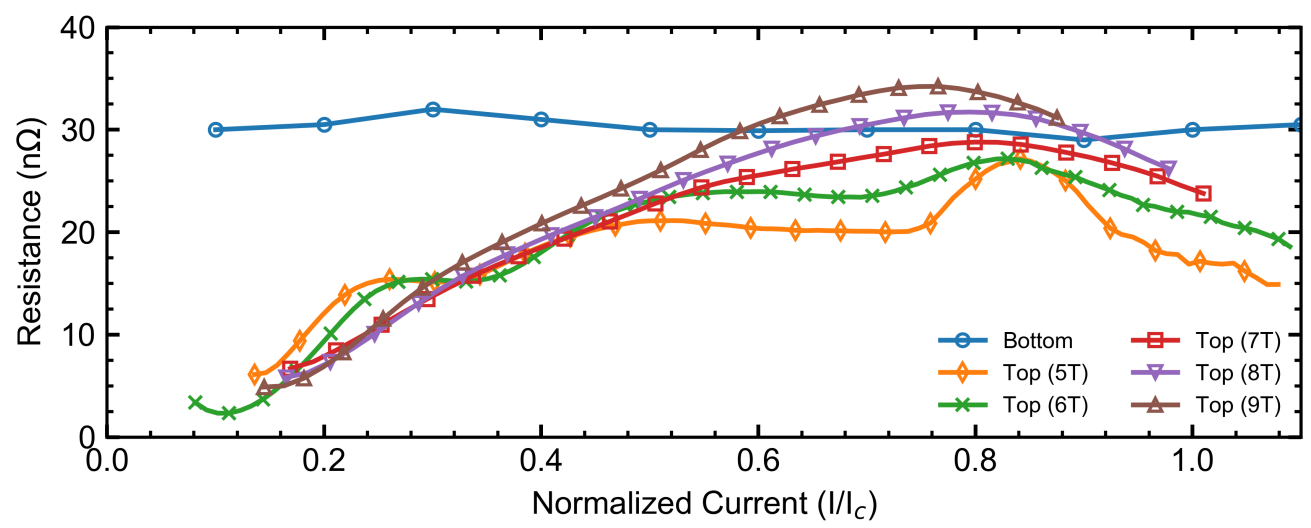

Figure 4.9: Resistance of the top and bottom joint terminals as function of normalized current for various magnetic fields. The resistance of the top terminal peaks at around $70 \%$ of the respective critical current after which it somehow decreases and appears to convert. This behavior can be explained by electro-magnetic forces acting on the cable and the joint terminal, which may improve either the contact resistance in the joint or improves current sharing between tapes. The bottom joint terminal has a higher, but more constant resistance for all measurement runs in various magnetic fields.

of after the quench is detected and the current is ramped down to $0 \mathrm{~A}$ in approximately $100 \mathrm{~ms}$. The total quench event takes about $300 \mathrm{~ms}$. The voltage rise is relatively slow and it takes several hundreds of milliseconds before a relatively large voltage is measured over the CORC sample. The normal zone propagates from the high field region to the joints and continues to the return leg. It is only when the return leg, comprising NbTi Rutherford cables, quenches in a fast en sudden manner, enough voltage is built up to trigger the quench protection system. The difference in quench behavior between LTS and HTS type conductors is clearly visible in the measurement results. The HTS CORC conductor quenches much slower since the critical current of the ReBCO tape is only slightly reduced with some kelvin increase in temperature, where the LTS NbTi conductor transitions from fully superconducting to fully normal-conducting in an instant. The slow rise in voltage over the CORC cable can be detected and current can then be decreased in time to prevent a quench.

Turning on the quench heaters locally reduces the critical current of the CORC cable by enhanced temperature. All outer four tapes respond more or less at the same time when one or both of the heaters are turned on, which indicates good heat distribution between tapes of the CORC conductor. No current redistribution over the tapes in the outer layer of the CORC sample is observed. Figure 4.11 shows the response of the CORC cable to a heat pulse using both heaters connected in parallel for almost $400 \mathrm{~ms}$. The conductor quenches around the time the heater is turned off. The normal zone propagates through the CORC sample and further heats the sample. The quench is detected and the current is ramped down approximately $150 \mathrm{~ms}$ after the heater is turned off. In this case, the normal zone does not reach the NbTi return leg, and only an inductive voltage is observed during 

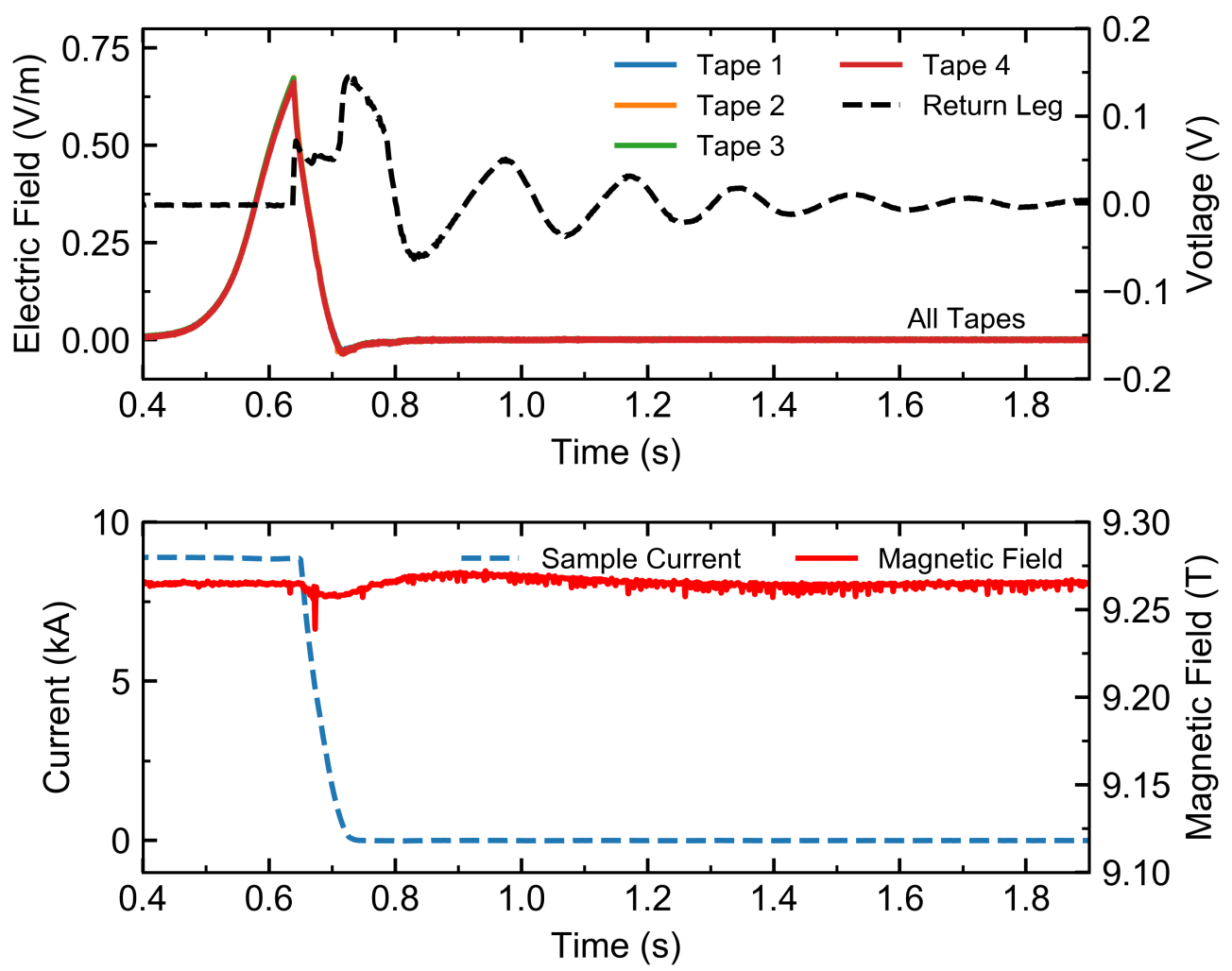

Figure 4.10: Top: Measured electric field of the ReBCO taps positioned on the outer layer tapes of the CORC sample and the voltage over the NbTi return leg as function of time. A slow and steady transition is observed in the CORC cable and a sharp transition is observed in the NbTi return leg that triggered the quench detection system. Bottom: The corresponding sample current and external magnetic field. Current is extracted in approximately $100 \mathrm{~ms}$.

ramping down of the current. The conductor is cooled directly in a bath of liquid helium and therefore no minimum quench energy measurement can be performed.

The CORC conductor survived all eleven quenches. Several control measurements were performed to check if degradation has occurred. The control measurements show minimal to no degradation in the CORC conductor, as shown in Figure 4.12. Only tape 1 in the outer layer shows some minor reduction in performance in the last two control measurements. All other tapes and also the full sample shows no clear signs of degradation. All control measurements are performed at $9 \mathrm{~T}$ and $4.2 \mathrm{~K}$ with a ramp-rate of $50 \mathrm{~A} / \mathrm{s}$. Note that the brass shunt across the sample works fine to protect the sample from burning out. 

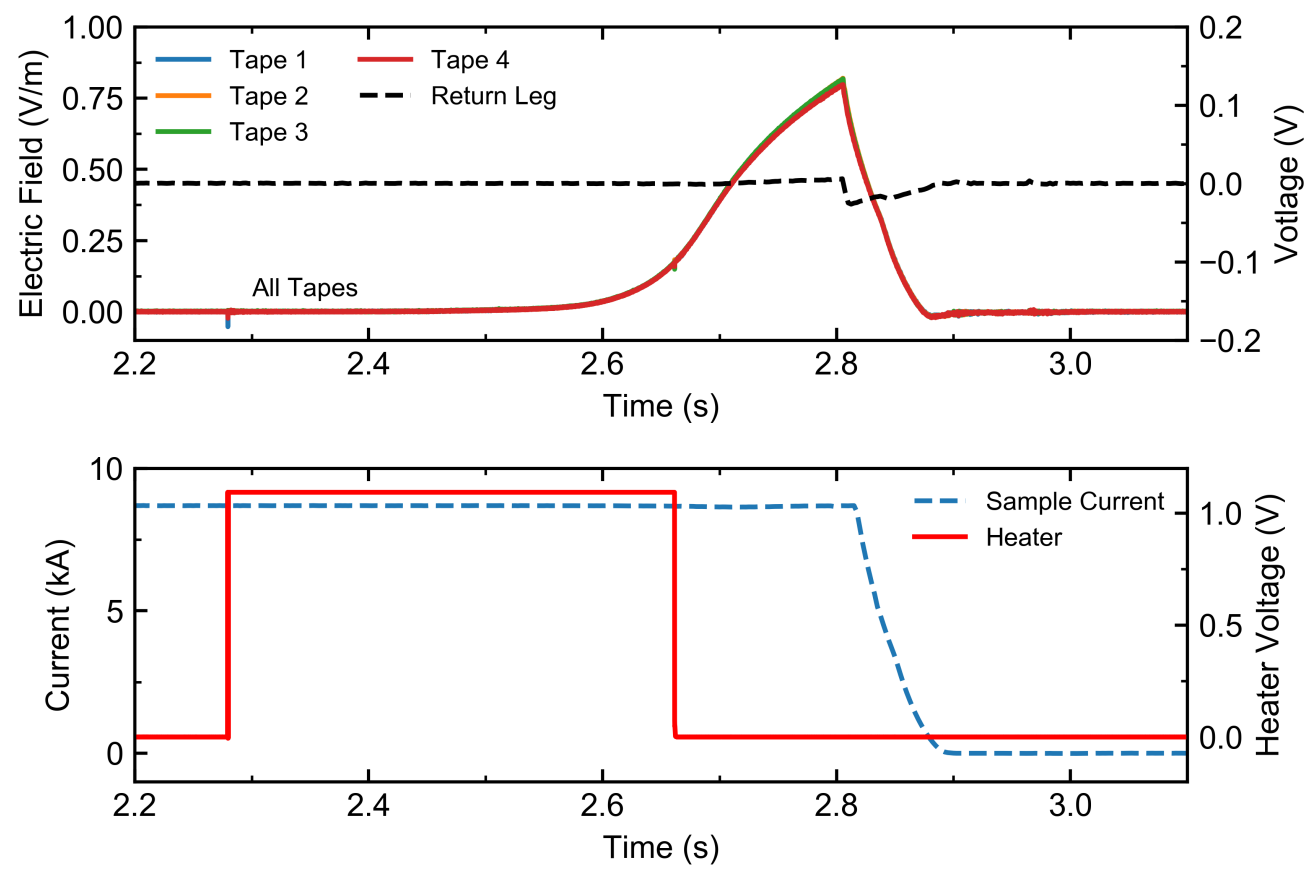

Figure 4.11: Top: Measured electric field of the ReBCO tapes located in the outer layer of the CORC sample and the voltage over the NbTi return leg as function of time. Bottom: Heater voltage and current as function of time. Heater is on for almost $400 \mathrm{~ms}$ and creates a normal zone in the CORC sample. After the heater is turned of, the normal zone is able to propagate for another $200 \mathrm{~ms}$ before the quench protection system is triggered.

\subsubsection{Discussion and Conclusion Regarding the CORC Cable Test}

A CORC cable comprising 38 ReBCO SCS4050 tapes was characterized at 1.9 and $4.2 \mathrm{~K}$ in an external magnetic field up to $9.6 \mathrm{~T}$. The benchmark critical current and critical current density at $10 \mathrm{~T}$ is $8.2 \mathrm{kA}$ and $174 \mathrm{~A} / \mathrm{mm}^{2}$, respectively. The measured critical current is very close the expected value in magnetic fields of 7 to $10 \mathrm{~T}$. The difference at lower magnetic field can be caused by the improved tape performance compared to the tapes of which the lift-factors were measured. After the CORC test, a new prediction was made using lift-factors obtained from more recent tests performed by J. Fleiter on Roebel cables [91] [92], which shows a better resemblance with the measured CORC data as is shown in Figure 4.13.

The joint terminals, although with a higher resistance than expected, show a more or less constant joint resistance instead of a resistance that rises with current. This means staging the joint terminal does indeed improve current sharing among ReBCO layers in the CORC conductor. The voltages over the four ReBCO tapes in the outer layer of the CORC cable do not overlap perfectly, which indicates that the current is still not perfectly distributed. One of the causes for imperfect current distribution between tapes in one single layer is 


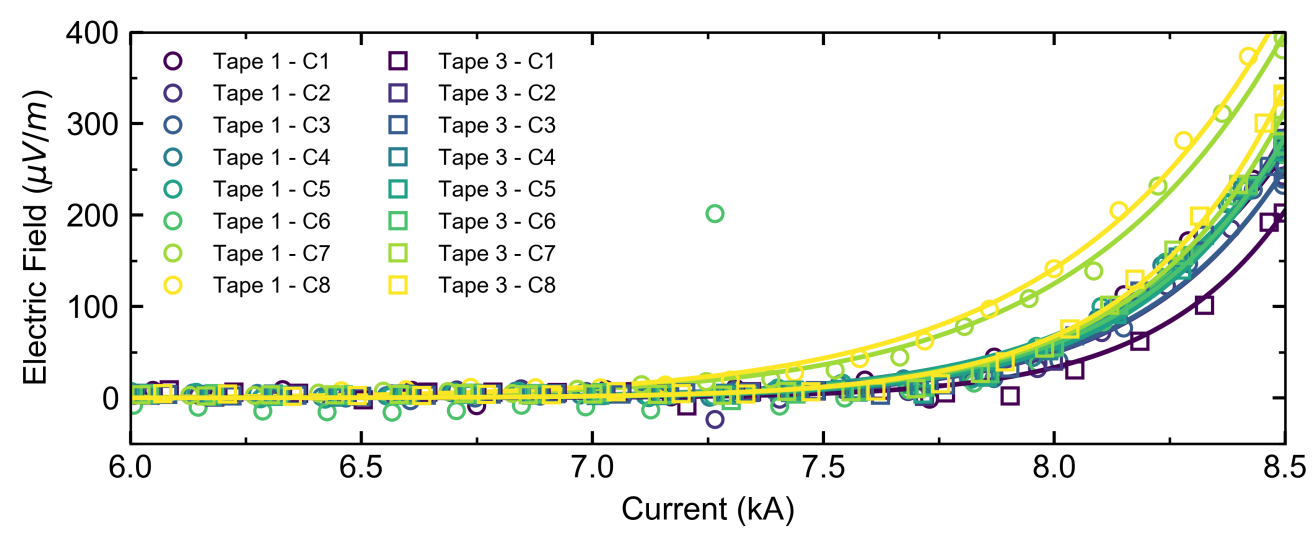

Figure 4.12: Reference measurements were performed to check if any degradation occurred during any of the measurements runs. The graph displays the electric field over the two worst performing monitored tapes in the outer layer as function of current at $9 \mathrm{~T}$ and a ramp-rate of $50 \mathrm{~A} / \mathrm{s}$. Tape 1 shows a minor decrease in critical current in the last two control measurements after the eleventh quench. There is no significant change observed in the overall voltage over the entire CORC sample.

not having a full twist pitch of the tapes within one of the stages in the joint terminal. This can lead to a variance in contact resistances between current lead and individual tapes and consequently a variance in tape currents. The current distribution in tapes in a single layer may be improved by having at least a full twist pitch for each tape within a single stage in the joint section, even if this means to have multiple layers within one stage (due to length limitations of the joint terminal).

There are three splices between the ReBCO tapes and the current lead; the tapes soldered to the cylindrical copper terminal, the cylindrical terminal to the copper intermediate block and the block to the NbTi current leads. This method is used to allow other CORC cables with similar cylindrical terminals to be compatible with the setup. In hindsight it might have been better to reduce the amount of splices in the joint by soldering the CORC cable directly to the intermediate block. The test does provide some indication of a poor soldering contact between the cylindrical CORC terminal and the copper block in which it is soldered. A good solder contact between copper block and the CORC cable can be assured by directly soldering the CORC sample to the copper block.

\subsection{Test of CORC Wires in Coil Configuration}

The reduction of ReBCO tape substrate thickness from 50 to $30 \mu \mathrm{m}$ achieved by SuperPower Inc. led to a reduction of the minimum, degradation free, bending diameter of the ReBCO tapes from 4.5 to $2.4 \mathrm{~mm}$ as described in detail in Section 2.2.2. This development made the preparation possible of thin CORC wires with diameters ranging from 3 to $4.5 \mathrm{~mm}$. Several CORC wires were prepared by Advanced Conductor Technologies and 


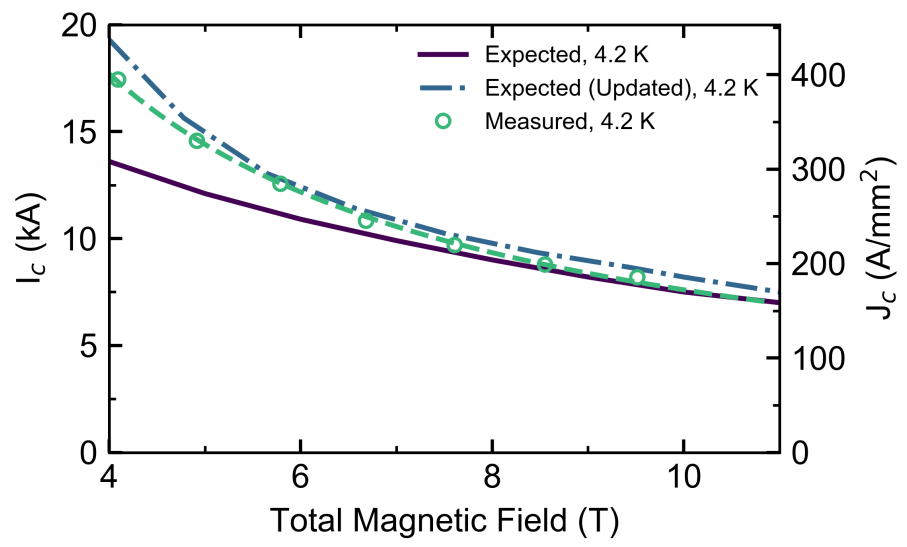

Figure 4.13: Measured critical current (left axis) and mean current density (right axis) as function of the total magnetic field (external + self-field) at 1.9 and $4.2 \mathrm{~K}$ with the updated expected $I_{c}$ at $4.2 \mathrm{~K}$ calculated with lift-factor values from [91][92].

tested at the University of Twente in a common research effort by CERN, ACT and the University of Twente. The aim of the experiments is to characterize, but also to demonstrate the performance and ease-of-use of CORC wires.

A test setup was designed and prepared at the University of Twente to demonstrate the flexibility of the CORC wires and measure their performance. The sample holder is a cylindrical stainless steel mandrel with an outer diameter of $60 \mathrm{~mm}$, which is characteristic for conductors used in research magnets and other applications where space is restricted. The wires are wound in several turns around the mandrel in the shape of a short solenoid. A picture of a wire on the sample holder is presented in Figure 4.14. The sample holder is inserted in a $\mathrm{Nb}_{3} \mathrm{Sn}$ solenoid providing up to $11 \mathrm{~T}$ background field. All tests were performed at $4.2 \mathrm{~K}$ in a bath of liquid helium. The self-field contribution in this setup is low compared to the background field as the self-field mainly points in the direction parallel to the tapes. The CORC wires included a $30 \mu \mathrm{m}$ thick layer of polyethylene insulation. The entire sample is wrapped with Teflon tape and coated with glass cloth and Stycast 2850FT epoxy resin to provide mechanical support in radial direction during the tests.

The effect of temperature and direction of the electro-magnetic load on the performance of the CORC wires was investigated as well. The wrapping of Teflon and fiber reinforced epoxy around the CORC samples not only provide mechanical protection, it also prohibits direct contact between the CORC wires and the bath of liquid helium. A wire heater wound around $20 \mathrm{~mm}$ of CORC wire is used to increase the temperature of the CORC wire locally as a gas pocket is created between CORC wire and wrap. The temperature is measured by a Cernox sensor placed between two turns of the CORC solenoid. The current sharing temperature $T_{c s}$ of the CORC wire at a fixed current and magnetic field is determined by increasing its temperature. The direction of the electro-magnetic loads in the sample depend on the direction of the sample current with respect to the magnetic 
field. The direction of the Lorentz force was maintained throughout the measurement campaigns of CORC wire samples to either inwards or outwards.

\subsubsection{CORC Wire Samples}

Four CORC wires were prepared by ACT for testing at the University of Twente. An overview of all CORC wires measured at the University of Twente is presented in Table 4.4. The wires are measured in several campaigns to allow intermediate feedback to optimize and improve the production and handling of CORC wires. The first wire, wire 1 , is 4.5 $\mathrm{mm}$ in diameter and comprises twenty-four $3 \mathrm{~mm}$ wide ReBCO SCS3030 tapes. The tape has the thin $30 \mu \mathrm{m}$ Hastelloy substrate, has $5 \mu \mathrm{m}$ of copper plating on each side and has a minimum bending diameter of $2.4 \mathrm{~mm}$. The second CORC wire, wire 2 , is thinner with a diameter of only $3 \mathrm{~mm}$ and comprises $162 \mathrm{~mm}$ wide ReBCO SCS2030 tapes with $5 \mu \mathrm{m}$ of copper stabilizer on each side of the tape. This wire is made to demonstrate the flexibility of thin CORC wires. The first two CORC samples were prepared in parallel and measured consecutively.

The third CORC wire comprises 50 of the $2 \mathrm{~mm}$ wide ReBCO tapes wound in 20 layers, a record amount of tapes in a single CORC wire. The inner most ReBCO layer is wound around the former of $1.9 \mathrm{~mm}$, which means that the tapes are wound around a diameter smaller than their minimum bending diameter. It was expected that the tapes in the inner layers would show degradation, but still retain a large fraction of their critical current.

The fourth wire is manufactured after feedback of the previous three wires. The wire comprises 29 of the $2 \mathrm{~mm}$ wide ReBCO tapes in 11 layers. The inner most layer is wound on a diameter of $2.4 \mathrm{~mm}$. The outer diameter of the wire is $3.5 \mathrm{~mm}$, which results in a modest flexible CORC wire.

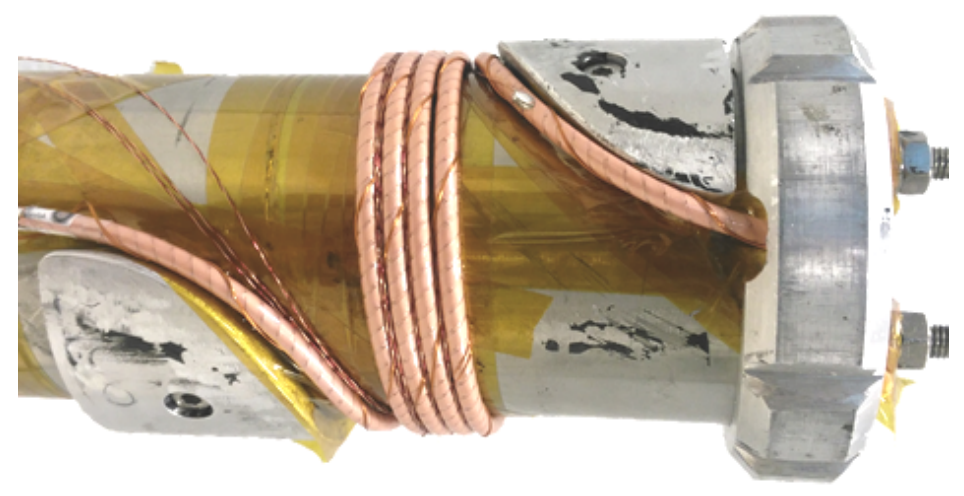

Figure 4.14: CORC wire wound into a 5-turn solenoid on the stainless steel sample holder prior to applying mechanical reinforcement. 
Table 4.4: Specifications of the CORC wire samples tested at the University of Twente.

\begin{tabular}{lccccc}
\hline \hline & Wire 1 & Wire 2 & Wire 3 & Wire 4 & Unit \\
\hline Production year & 2016 & 2016 & 2016 & 2017 & - \\
Number of tapes & 24 & 16 & 50 & 29 & - \\
Number of layers & 12 & 8 & 20 & 11 & - \\
Tape type & SCS3030 & SCS2030 & SCS2030 & SCS2030 & - \\
Outer diameter & 4.5 & 3 & 4.1 & 3.5 & $\mathrm{~mm}$ \\
Core material & $\mathrm{Cu}$ & $\mathrm{Cu}$ & $\mathrm{Cu}$ & $\mathrm{Cu}$ & - \\
Core diameter & 3.0 & 2.4 & 1.9 & 2.4 & $\mathrm{~mm}$ \\
\hline \hline
\end{tabular}
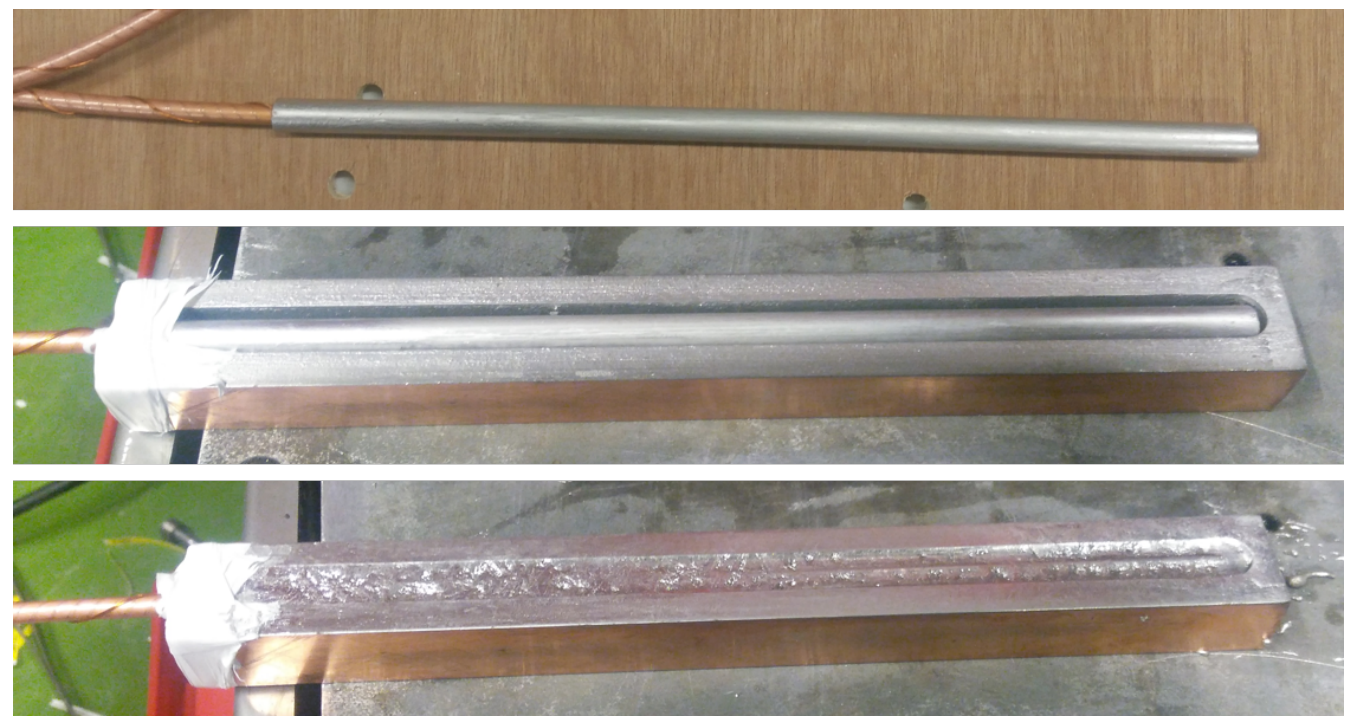

Figure 4.15: Cylindrical joint terminal of a CORC wire soldered to a rectangular copper block, which is connected to the current lead of the setup.

The cylindrical joint terminals for the wires were prepared by ACT by tapering each layer. Each cylindrical terminal is 150 to $180 \mathrm{~mm}$ long and soldered into a rectangular copper block. The connection between the cylindrical terminal and the rectangular leads is made by an intermediate copper block filled with In-Sn-Cd solder. This is a low-resistance method to connect cylindrical joint terminals to rectangular leads. A picture of this type of connection is presented in Figure 4.15. The voltage taps were placed on the outer copper casing of the terminal and about $50 \mathrm{~mm}$ onto the CORC wire. The resistance of the terminals includes the additional solder connection between the rectangular copper casing and the cylindrical copper tube that is fitted to the CORC wire. In some cases there are additional voltage taps inside the cylindrical terminal, which were inserted and soldered together with the CORC cable. The predicted joint resistance of the joints is 12 to $13 \mathrm{n} \Omega$ depending on the precise configuration. 


\subsubsection{Degradation of the First Sample}

The first CORC wire, comprising $3 \mathrm{~mm}$ wide tapes, showed signs of buckling in the last bend on the sample holder. The first bend and the spiral showed minimal to no signs of buckling. A possible explanation that buckling only occurred in the last turn of the sample holder is that the stress caused by bending the CORC wire in the first turn and in the spiral moved along the the wire and only expressed itself after the stress in the tapes reached a certain threshold. The polyethylene sleeve was removed from the entire CORC wire during the bending procedure for practical access to the ReBCO tapes in the outer layer. Leaving the insulation sleeve on the CORC conductor probably helps distributing stress better over the conductor length as it restricts movement of tapes in the outer layers. A copper shunt is soldered over the section with the buckled tapes to provide a parallel path for the current and allow a measurement of the conductor's critical current. However, the degraded section transitioned from superconducting to normal state and eventually caused a quench in the sample before the critical current of the spiral could be reached. The tapes of this CORC wire are $3 \mathrm{~mm}$ wide, likely too wide for such tight bending. The degradation observed in the wire was used as feedback for improving the sample holder and the next CORC wires. All next CORC wires have tapes of $2 \mathrm{~mm}$ in width and their polyethylene sleeve is not removed.

\subsubsection{Electrical Performance of the CORC Wires}

The measured critical current densities of wires 2, 3 and 4 are presented in Figure 4.16. The criterion to determine the critical current is $1 \mu \mathrm{V} / \mathrm{cm}$. CORC wire 2 is the thinnest CORC wire in the measurement program. The wire reached a critical current of $2560 \mathrm{~A}$ at $10 \mathrm{~T}$, which corresponds to a current density of $362 \mathrm{~A} / \mathrm{mm}^{2}$. This is not a record performing wire at a magnetic field of $10 \mathrm{~T}$, however, it demonstrated for the first time the feasibility of flexible thin CORC wires and their ease of use.

CORC wire 3 shows a critical current of $4255 \mathrm{~A}$ at $10 \mathrm{~T}$ and $4.2 \mathrm{~K}$ with an n-value of $30 \pm 15$, corresponding to an overall engineering current density $J_{e}$ of $322 \mathrm{~A} / \mathrm{mm}^{2}$. CORC wire 4 has a critical current of $3970 \mathrm{~A}$ at $10 \mathrm{~T}$ and $4.2 \mathrm{~K}$ with an $\mathrm{n}$-value of $32 \pm 7$, corresponding to a current density of $412 \mathrm{~A} / \mathrm{mm}^{2}$. The performance of both wires is lower than expected and both wires have very similar measured critical currents, although one wire comprises much more tapes. It is concluded that CORC wire 3 shows significant degradation imposed by the coil winding on the sample holder.

Tape samples from several tape batches used for winding the CORC wires were tested afterwards at Florida State University to determine the tape's lift factor at $4.2 \mathrm{~K}$ in magnetic fields between 6 and $15 \mathrm{~T}$. The lift factor is 1.8 at $10 \mathrm{~T}$, defined as $\mathrm{I}_{c}(10 \mathrm{~T}, 4.2 \mathrm{~K}) / \mathrm{I}_{c}$ (selffield, $77 \mathrm{~K}$ ), for the batch of tapes used for CORC wire 4 . This value, determined by the ReBCO manufacturing process, is relatively low, compared to the typical lift factor of 2.7, which would correspond to a wire current density of about $600 \mathrm{~A} / \mathrm{mm}^{2}$ at $10 \mathrm{~T}$ and $4.2 \mathrm{~K}$. Batches of tape have been delivered to ACT by SuperPower in 2016 with lift factors as high as 3.4, which would have resulted in a wire current density of over $800 \mathrm{~A} / \mathrm{mm}^{2}$ at $10 \mathrm{~T}$ and $4.2 \mathrm{~K}$. 


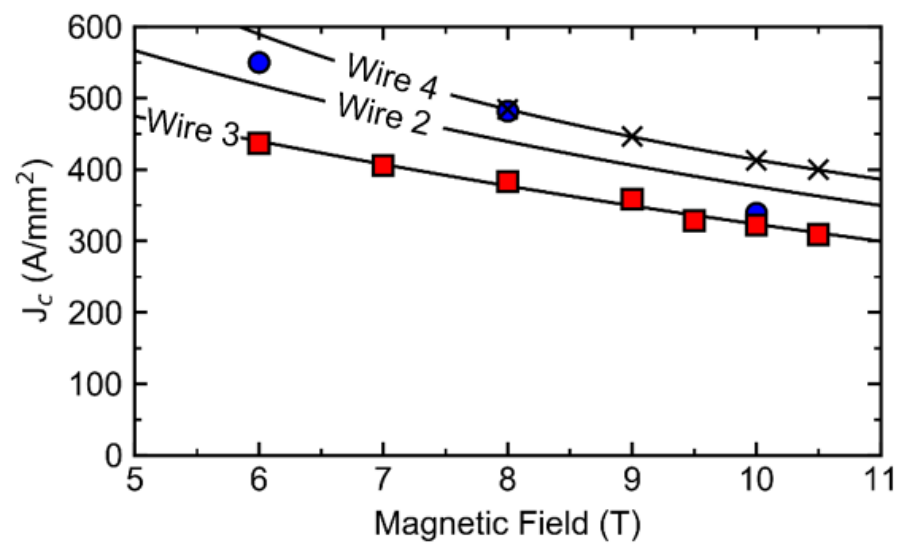

Figure 4.16: Measured critical current density (markers) and its fit (lines) for CORC wires 2, 3 and 4 as function of magnetic field at $4.2 \mathrm{~K}$ (see Table 4.4 for wire description).

\subsubsection{Effect of Electro-Magnetic Load Cycling}

The Lorentz force on CORC wires 2 and 3 pointed inwards during the measurements and the Lorentz force on CORC wire 4 pointed purposely outwards in order to assess any change in wire performance due to the direction of the Lorentz force. CORC wire 3 shows a reduction in critical current after each load cycle. The measured E(I) curves of the wire are presented in Figure 4.17 and the critical current as function of magnetic field and run number are shown in Figure 4.19. The critical current did reduce by approximately $10 \%$ of its original $\mathrm{I}_{c}$ after 12 runs due to electro-magnetic load cycling (at several applied magnetic fields). The current sharing starts around the same current in all runs, therefore the n-value increases as function of run number as the critical current decreases. Degradation of CORC wire 2, with the Lorentz forces also pointing inwards, was not observed. However, current and thus the forces in CORC wire 2 are much lower compared to CORC wire 3 . Wire 4 did not show such degradation as the critical current did not change per cycle as shown in Figure 4.18 and Figure 4.19. The much better performance under stress cycling of wire 4 is a direct result of the winding configuration that ensured the strain state of each tape remaining below the critical strain limit during coil winding and highfield operation. As discussed in the next section, the strain state of many tapes in wire 3 already exceeded the critical strain limit during coil winding [65, 22], while the added Lorenz force during testing, with the force pointing inward, pushed the strain state of the tapes even further and over their limit.

\subsubsection{Analyses of Extracted Tapes}

Tapes in wires 3 and 4, that were positioned in the center of the high magnetic field region, were extracted by ACT after the test at the University of Twente. Each tape was tested individually to assess any degradation and to pinpoint the layer in which the degradation 


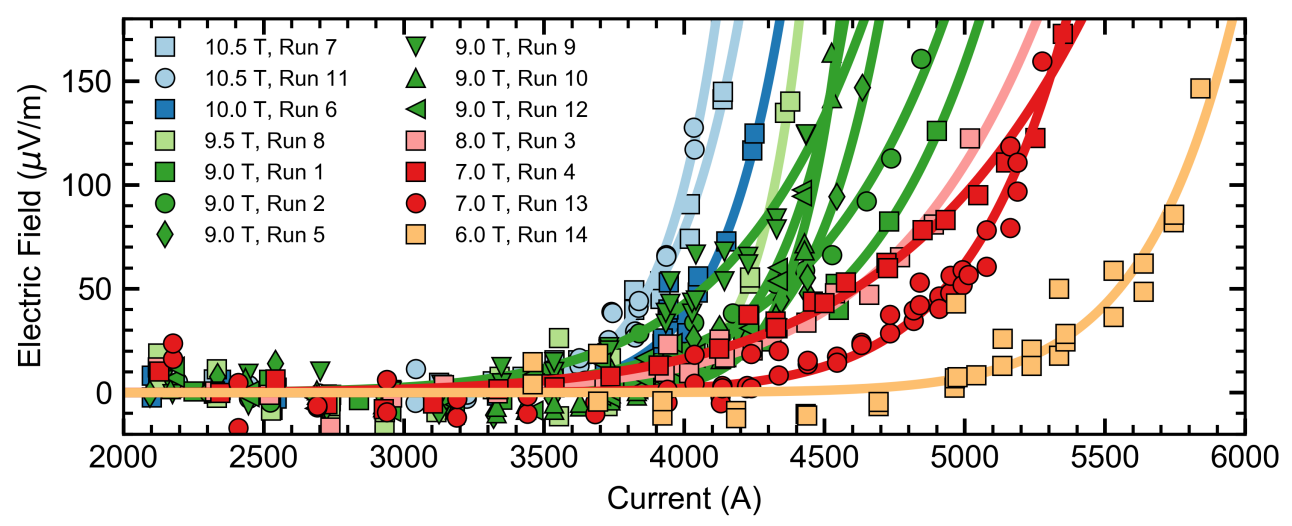

Figure 4.17: Electric field over the CORC spiral as function of current. Critical current $I_{c}(B, 4.2 \mathrm{~K})$ measurements on CORC wire 3 show a clear sign of degradation in critical current likely due to electromagnetic load cycling with the Lorentz forces pointing inwards.

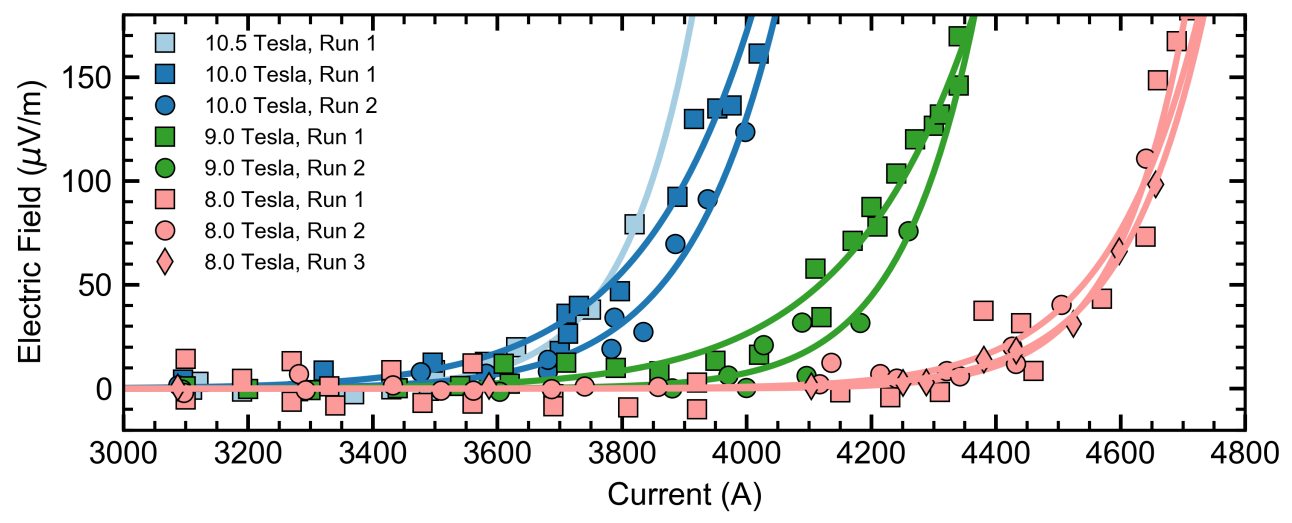

Figure 4.18: Electric field over the CORC spiral as function of current. Critical current $I_{c}(B, 4.2 \mathrm{~K})$ measurements on the CORC wire 4 show no degradation due to electro-magnetic load cycling with Lorentz forces pointing outwards.

occurred. The critical current of each tape was measured at $76 \mathrm{~K}$ in self field and compared to the performance of a virgin tape from the same batch.

CORC wire 3 showed large degradation in tapes extracted from the inner most layers, as shown in Figure 4.20. This is a clear indication of the winding diameter of the inner layers being too small and the ReBCO layer in the tapes experiencing too much compressive strain. This degradation can be expected as the strain dependence of ReBCO tapes has been well documented in literature [65][22].

It also confirms the minimum tape bending diameter of $2.4 \mathrm{~mm}$ even after the CORC wires are bent. However, the partially degraded tapes still provide a significant contribution 

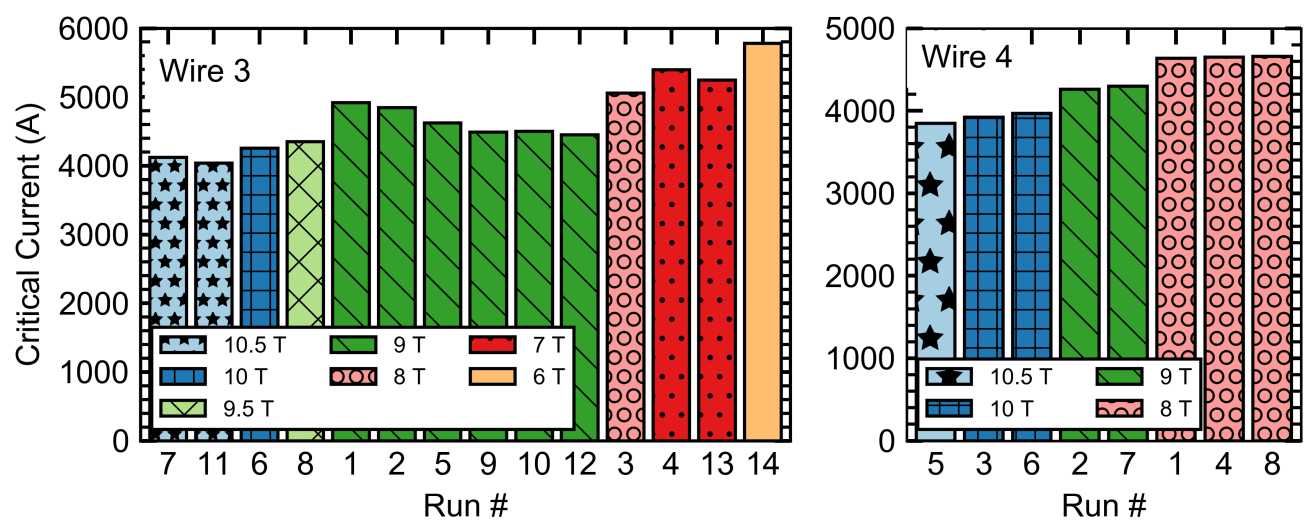

Figure 4.19: Critical current as function of magnetic field and run number for CORC wires 3 and 4. A decrease in critical current of CORC wire 3 is observed with each subsequent measurement run, which indicates degradation due to the electro-magnetic load cycling. No degradation is observed due to the electro-magnetic load cycles for CORC wire 4, which had the Lorentz forces pointed outwards instead of inwards.
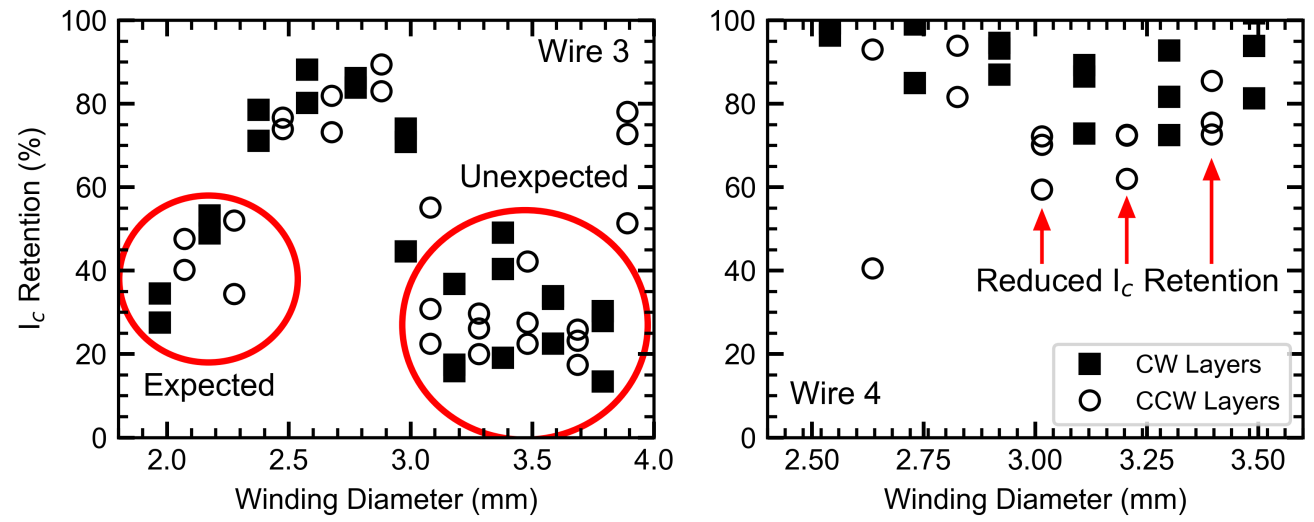

Figure 4.20: Critical current retention versus winding diameter of the extracted tapes from wires 3 (left) and 4 (right) measured by ACT. The single tapes extracted from the 50 tape CORC wire after the test at the University of Twente shows degradation in the layers with a winding diameter less than $2.4 \mathrm{~mm}$ as expected. Unexpected degradation was observed in the outer layers, likely caused by insufficient tape winding tension during wire production. Only some minor $I_{c}$ reduction is observed in each other ReBCO layer in CORC wire 4, suggesting the CORC wire was lightly torqued.

to the wire's critical current. The rather severe degradation of tapes in the outer layers was not expected. The outer nine layers have a critical current retention of around 30\%, which is likely caused by a too low tape winding tension during the wire production. If winding tension is too low in wires with a large $(>9)$ number of layers, there is enough accumulated slack for tapes to deform rather than slide when the wire is bent, resulting 
in macroscopic kinking, $R e \mathrm{BCO}$ layer cracking and poor $\mathrm{I}_{c}$ retention. The tension needs to be adjusted for wire production to be sufficiently high for intimate contact between tapes, but still kept low enough to not damage tapes and to allow tapes to slide when the conductor is bent. The total $\mathrm{I}_{c}$ retention of CORC wire 3 was $48 \%$.

CORC wire 4 has a much higher $\mathrm{I}_{c}$ retention of $82 \%$, but still showed some minor tape degradation, as presented in Figure 4.20. The observed degradation is different than observed in wire 3. Every other layer shows a higher $\mathrm{I}_{c}$ retention, suggesting either the wire was torqued during wire bending (every other layer either tightened or loosened) or the tape tension was different when winding tapes in clockwise (CW) direction versus counterclockwise (CCW) direction. Accounting for both the lower lift factor and the observed $\mathrm{I}_{c}$ retention in the extracted tapes, the expected current density at $10 \mathrm{~T}$ and $4.2 \mathrm{~K}$ is reduced to $400 \mathrm{~A} / \mathrm{mm}^{2}$, which is very close to the measured $412 \mathrm{~A} / \mathrm{mm}^{2}$.

\subsubsection{Effect of Temperature on Wire Performance}

The effect of temperature is studied by heating CORC wire 2 with a heater. The CORC wire is isolated from the liquid helium by the Teflon and Stycast layers. Helium between the CORC wire and the Stycast layer evaporates and creates a 'warm' gas pocket when the heater is on. Current sharing temperature $T_{c s}$ measurements were performed with a conductor current of $1 \mathrm{kA}$. The resulting electric field over the CORC wire as function of temperature and magnetic field is presented in Figure 4.21. At $10 \mathrm{~T}$ the $\mathrm{T}_{c s}$ is $10.7 \mathrm{~K}$. Extrapolating the measured value at $10 \mathrm{~T}$ to 8, 6, 4, 2 and $0 \mathrm{~T}$ external field yields a $\mathrm{T}_{c s}$ of $16.3,22.3,34.7,47.5$, and $72.8 \mathrm{~K}$, respectively. The self-field of the sample operating at $1 \mathrm{kA}$ is in the order of tenths of $\mathrm{mT}$ and therefore doesn't contribute significantly to the total magnetic field. The measured $T_{c s}$ values at external magnetic fields of $0,2,4,6$ and $8 \mathrm{~T}$ are not corresponding well to the expected values. The $0 \mathrm{~T}$ and $2 \mathrm{~T}$ (only self-field) data even overlap. Apparently, there is a large temperature gradient over the CORC wire and the hot-spot temperature is actually much higher than the measured temperature. It also means that the copper core of the CORC wire provides sufficient cooling to the ReBCO tapes as transition to normal state is a slow process in the order of dozens of seconds and over a temperature range of many kelvin. This demonstrates the large stability of ReBCO based conductors compared to LTS conductors.

\subsubsection{Joints for CORC wires}

The joints of CORC wire 3 have a resistance of 12 to $23 \mathrm{n} \Omega$ as presented in Figure 4.22. The joint resistance is $22.9 \mathrm{n} \Omega$ for one joint and $17.1 \mathrm{n} \Omega$ for the second at low currents in the range of 0 to $2500 \mathrm{~A}$. A distinct kink in the curve is observed around $2500 \mathrm{~A}$, slightly depending on the external magnetic field, after which the joint resistance decreases to respectively $14.2 \mathrm{n} \Omega$ and $11.7 \mathrm{n} \Omega$. It is possible that the electro-magnetic forces improve current distribution by locally improving the strain dependent critical current of the $\mathrm{ReBCO}$ tapes in the high magnetic field region. Another possibility is that the resistance of the electrical connection from the current leads of the setup, which are partially in the stray field of the magnet, decreases due to the electromagnetic forces and thereby 


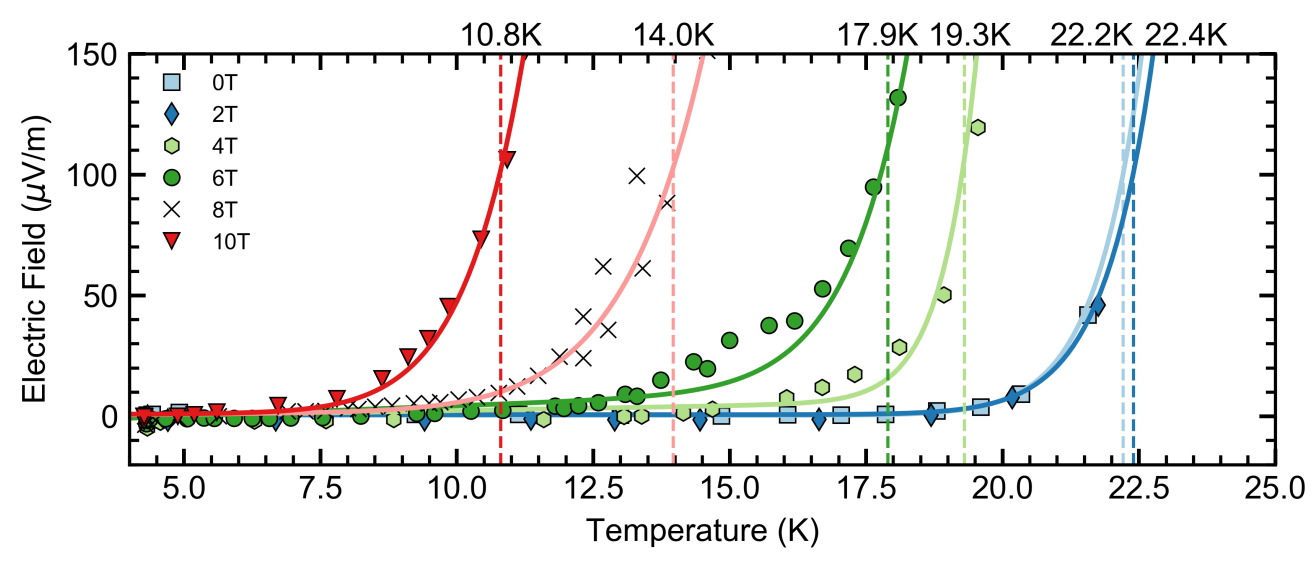

Figure 4.21: Electric field in CORC wire 2 as function of measured temperature for various external magnetic fields with $1 \mathrm{kA}$ of sample current.

improves the current distribution in the terminals. The predicted joint resistance of these joints is in the range of $12 \mathrm{n} \Omega$, which is very close to the measured values.

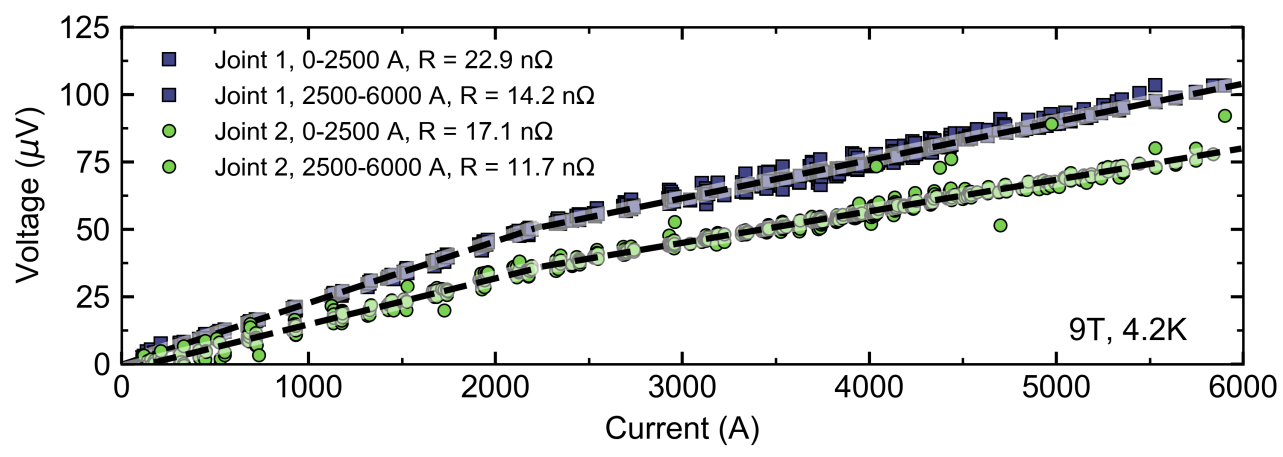

Figure 4.22: Joint resistance of the first wire as function of current showing $23 n \Omega$ initially and $12 \mathrm{n} \Omega$ beyond some $2.5 \mathrm{kA}$. A clear change in resistance was observed around the $2500 \mathrm{~A}$ mark (slightly different as function of magnetic field), after which the resistance drops with about $6 \mathrm{n} \Omega$.

The joint terminals of CORC wire 4 show a rather high but constant resistance of $34 \pm 2 \mathrm{n} \Omega$ and $53 \pm 2 \mathrm{n} \Omega$ in various background magnetic fields and a temperature of $4.2 \mathrm{~K}$, as shown in Figure 4.23. The voltage taps inside the copper tube of the terminals show an average joint resistance of only $5 \pm 1 \mathrm{n} \Omega$. Therefore, it is likely that the observed high resistance is located between the cylindrical terminal and the current leads. The $5 \pm 1 \mathrm{n} \Omega$ is much less than the expected $13 \mathrm{n} \Omega$. However, this resistance does not include the resistance from current lead to the inner bore of the copper cylinder, which is calculated to be around $3 \mathrm{n} \Omega$. In all cases, the resistance is very constant over the entire current range, which indicates that the staging of the terminal does distributed current evenly over the different tapes 
in the various layers of the CORC wire. Later characterization of these joint terminals without magnetic field in liquid nitrogen showed a low full-sample resistance of $46 \mathrm{n} \Omega$ at $76 \mathrm{~K}$. The average joint resistance of $23 \mathrm{n} \Omega$ at $76 \mathrm{~K}$ is slightly below the predicted $27 \mathrm{n} \Omega$, caused by the temperature dependent increase of copper and solder resistivity. Therefore, it is likely that the high resistance observed at the $4.2 \mathrm{~K}$ measurements is caused by an imperfect splice between current leads the copper joint blocks.
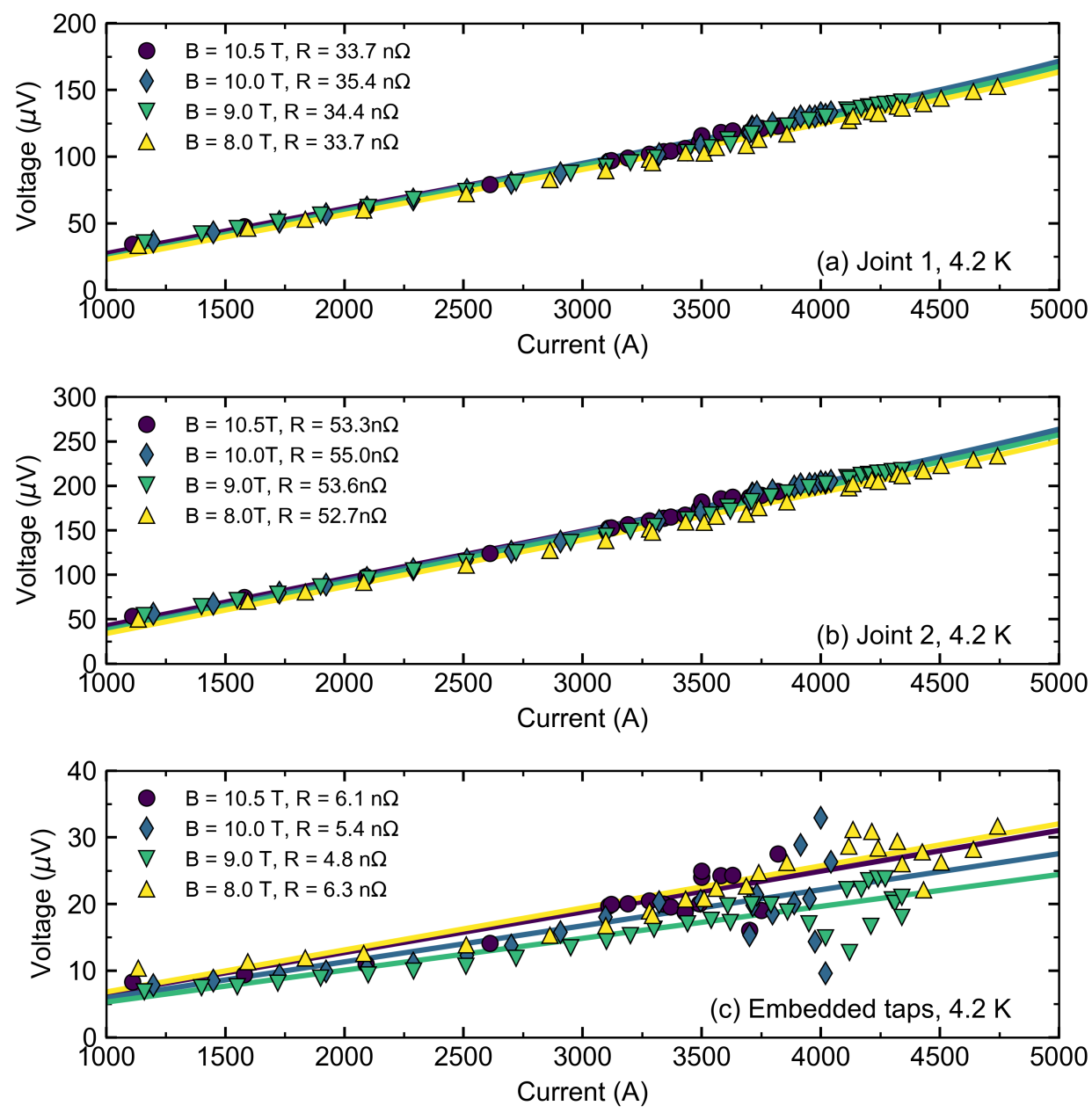

Figure 4.23: Joint resistance measured on the joints of the fourth CORC wire. The top and middle graphs show the resistance measured directly over the joints, which includes the resistance of the splice of the cylindrical terminal to the intermediate copper block. The resistance of both terminals is much larger than the expected 10 to $12 \mathrm{n} \Omega$. The bottom graph shows the resistance measured using voltage taps that are soldered into the terminal together with the CORC cable. These voltage taps show a much lower resistance, which indicates that the high resistance measured using the other voltage taps is likely due to the connection between cylindrical terminal and intermediate copper block. 


\subsubsection{Conclusion}

Four experimental CORC wires were prepared by ACT for characterization at the University of Twente. One of the four wires, the wire with the $3 \mathrm{~mm}$ wide tapes, degraded according expectation due to bending and could not be accurately characterized. The other three CORC wires demonstrated high current densities of 320 to $420 \mathrm{~A} / \mathrm{mm}^{2}$ at $10 \mathrm{~T}$ and $4.2 \mathrm{~K}$.

CORC wire 3 has a critical current of $4255 \mathrm{~A}$ at $10 \mathrm{~T}$ and $4.2 \mathrm{~K}$, which corresponds to an engineering current density of $322 \mathrm{~A} / \mathrm{mm}^{2}$. This CORC wire showed $10 \%$ degradation due to electro-magnetic load cycles explained by the strain state of a large number of the tapes exceeding the critical strain limit after sample coil winding, and further enhanced by the Lorenz force during testing. Extracted tape analysis afterwards showed that the additional degradation is likely caused by insufficient tape winding tension during wire production. The resulting $\mathrm{I}_{c}$ retention of wire 3 is $48 \%$.

CORC wire 4 demonstrated a critical current of $3970 \mathrm{~A}$ at $10 \mathrm{~T}$ and $4.2 \mathrm{~K}$, corresponding to an engineering current density of $412 \mathrm{~A} / \mathrm{mm}^{2}$. This wire shows no degradation due to load cycling. The tapes used in CORC wire 4 has a low lift-factor. The wire performance could have been a factor 2 better if tapes with a higher lift factor were used. The extracted tape analysis shows that the total $\mathrm{I}_{c}$ retention of wire 4 is $82 \%$.

The minimum bending diameter of $2.4 \mathrm{~mm}$ for the ReBCO tapes with $30 \mu \mathrm{m}$ of Hastelloy substrate has been clearly demonstrated by extracting and testing single tapes from each CORC wire. The minimum bending diameter of the ReBCO tapes can be further decreased by positioning the ReBCO layer within the tape closer to its neutral axis by either reducing substrate thickness or by copper plating only one side of the tape. Such development is beneficial for both the flexibility and current density of CORC wires. CORC wires comprising ReBCO coated conductors with a centered ReBCO layer are currently under development $[93,94]$ and can provide an important boost to CORC wire technology.

The joints of CORC wire 3 have a resistance of 14 and $12 \mathrm{n} \Omega$, according to prediction, and the joints of wire 4 have a resistance of 34 and $53 \mathrm{n} \Omega$. The resistance values for the joints of wire 4 are higher than expected, but seems to be caused by the splice between current leads and the cylindrical terminal. At $76 \mathrm{~K}$, these terminals show the predicted resistance. Therefore, the joint model, described in Section 3.3, has provided good indication of joint resistance.

The tests have provided valuable feedback for further optimization and improvement of the production and handling of CORC wires to arrive at much higher level of performance.

\subsection{ReBCO CORC Demonstration Magnets}

ReBCO coated conductors currently have the highest critical current and critical field at $4.2 \mathrm{~K}$ of commercial superconductors and surpass by far common LTS conductors, 
such as $\mathrm{NbTi}$ and $\mathrm{Nb}_{3} \mathrm{Sn}$ based cables, in performance. ReBCO coated conductors allow realization of high-field magnets capable of generating magnetic fields far beyond $20 \mathrm{~T}$. The development of ReBCO based magnets and hybrid LTS/HTS magnets is progressing at a significant pace. In recent years several ReBCO based magnets have been presented by institutes from all over the globe demonstrating the wide interest in the ReBCO magnet technology.

\subsubsection{Current Status of ReBCO Coated Conductor based Magnets}

ReBCO tape allows practical bending in one direction only. Therefore, the most practical shape for ReBCO tape is a flat pancake coil, either in circular fashion as part of a multilayer solenoid or racetrack coil. A recent example of a high-field magnet using ReBCO CC pancake coils is the $32 \mathrm{~T}$ hybrid magnet of the NHFML[15]. The magnet comprises an inner HTS solenoid assembly of 112 pancakes and an LTS NbTi and $\mathrm{Nb}_{3} \mathrm{Sn}$ outer solenoid. The outer coils generate $15 \mathrm{~T}$ and the inner ReBCO solenoid boosts the magnetic field to $32 \mathrm{~T}$ and is world's first HTS user magnet that can demonstrate operation beyond $30 \mathrm{~T}[95]$.

HTS magnets solely based on single ReBCO tapes have branched to the direction of insulated coils, such as the $32 \mathrm{~T}$ magnet of the NHFML, where all turns are insulated. But also in the direction of HTS magnets were no turn-to-turn insulation is present. These 'no-insulation coils' have the advantage of self-protection against quenches, within certain limits, as the current can bypass a quenched region by redistributing current directly to subsequent turns and layers. The downside of this concept is that it can be used for quasistationary operation only at relatively low voltage during ramp-up and ramp-down, such that the majority of the current flows in the superconductor and not passes directly from layer to layer. Demonstration of two of such magnets is presented by S. Yoon et al. in [96] and J. Song et al. in [97].

\section{ReBCO Multi-Strand Conductors for High-Field Magnets}

Cabling ReBCO tapes to create a multi-strand high-current conductor is beneficial for simplifying magnet design, protection, generally improves stability and reduces consequences of damaged tapes since many tapes are in parallel. Multi-strand ReBCO conductors such as Roebel and CORC are therefore requested for high-field magnets. Both Roebel cable and CORC wire have been implemented in demonstration dipole magnets. The ReBCO Twisted Stacked cable variant doesn't have a bending radius small enough to allow high-field magnets with small dimensions, although some design studies have been conducted [100]. Instead, the Twisted Stacked conductor development is addressing mainly large scale magnets, i.e. magnets for an experimental fusion reactor.

CERN has prepared two dipole demonstrator magnets within the EuCARD program using the ReBCO Roebel cable technology called Feather-M0 and Feather-M2 [101, 27]. A picture of the assembled Feather-M2 magnet is presented in Figure 4.24. The Feather magnets are of the aligned-to-parallel block type and developed to operate as insert for a 


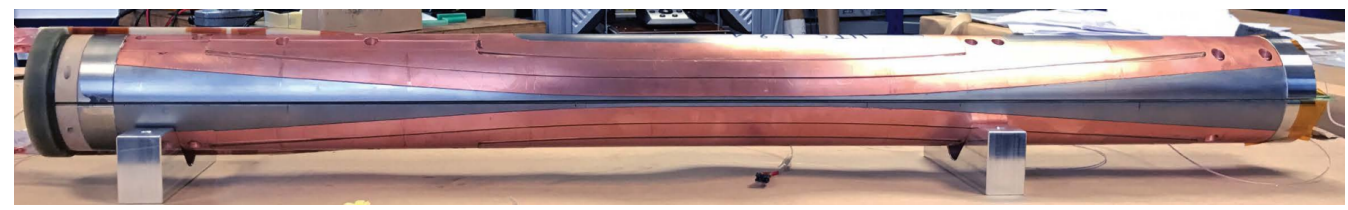

Figure 4.24: Sideview of the Feather M2 magnet assembled at CERN [98].
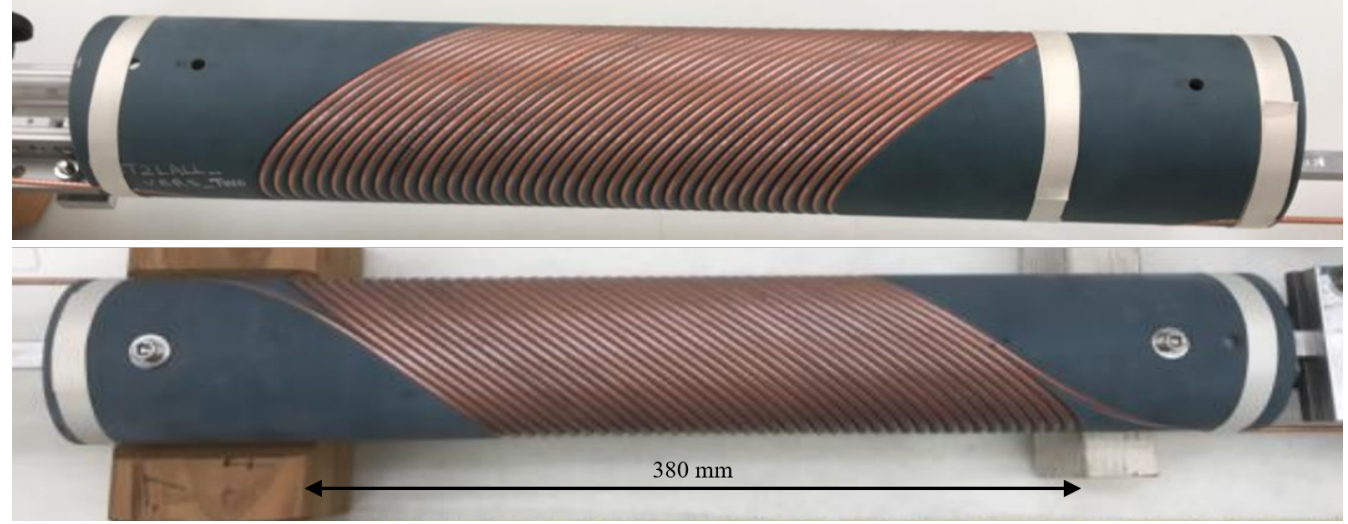

Figure 4.25: Outer and inner layer of the multi-turn CORC CCT that is under development at the Lawrence Berkeley National Laboratory [99].

20 T high-field dipole magnet. The high current density of the Roebel cable in combination with optimal conductor alignment within the magnetic field lines allows a very efficient design with a minimum of conductor. Both Feather magnets have been tested showing the feasibility of ReBCO Roebel cables for high field magnets [98].

The first CORC type dipole magnet is being developed at Lawrence Berkeley National Laboratory [99]. The magnet is of the type Canted-Cosine-Theta (CCT) and is wound with state-of-the-art thin CORC wire. The CCT shape is well matched to the round shape of CORC wire. The first demonstrator CORC CCT coil with only a few turns was tested successfully. At the time of writing, a 40-turn CORC CCT is being prepared, as is shown in Figure 4.25.

\subsubsection{CORC Racetrack Coil at CERN}

A CORC type racetrack coil is under development at CERN to demonstrate the feasibility of CORC wires in high-field dipole magnets. The racetrack coil shape implies the challenge of bending CORC wires on small bending radii with high magnetic field, high currents and high electro-magnetic loads. A short CORC racetrack trial coil is prepared and tested by S. Rindt et al. [102] to provide a proof of concept.

The racetrack design comprises 2 layers of CORC wire with each 8 turns. The racetrack has a bore of $40 \mathrm{~mm}$ in diameter, which is $20 \mathrm{~mm}$ less than the sample holder on which CORC wires were tested at the University of Twente. The length of the magnet is $190 \mathrm{~mm}$ 
as presented in Figure 4.26. The racetrack coil will be tested in the FRESCA cable test facility at CERN. Fresca has a cold bore of $71 \mathrm{~mm}$ in diameter, which is constraining the size of the coil.

The coil self-inductance is $50 \mu \mathrm{H}$ and it can generate a magnetic field of $380 \mathrm{mT}$ per $1000 \mathrm{~A}$. The stand alone, racetrack is expected to have a critical current of almost $10 \mathrm{kA}$ at $4.2 \mathrm{~K}$, producing a $3.7 \mathrm{~T}$ peak field on the conductor and a $2.3 \mathrm{~T}$ central field. In the background magnetic field of $9.6 \mathrm{~T}$ of the FRESCA facility the racetrack can generate an additional $2 \mathrm{~T}$ for a total magnetic field of $11.6 \mathrm{~T}$.

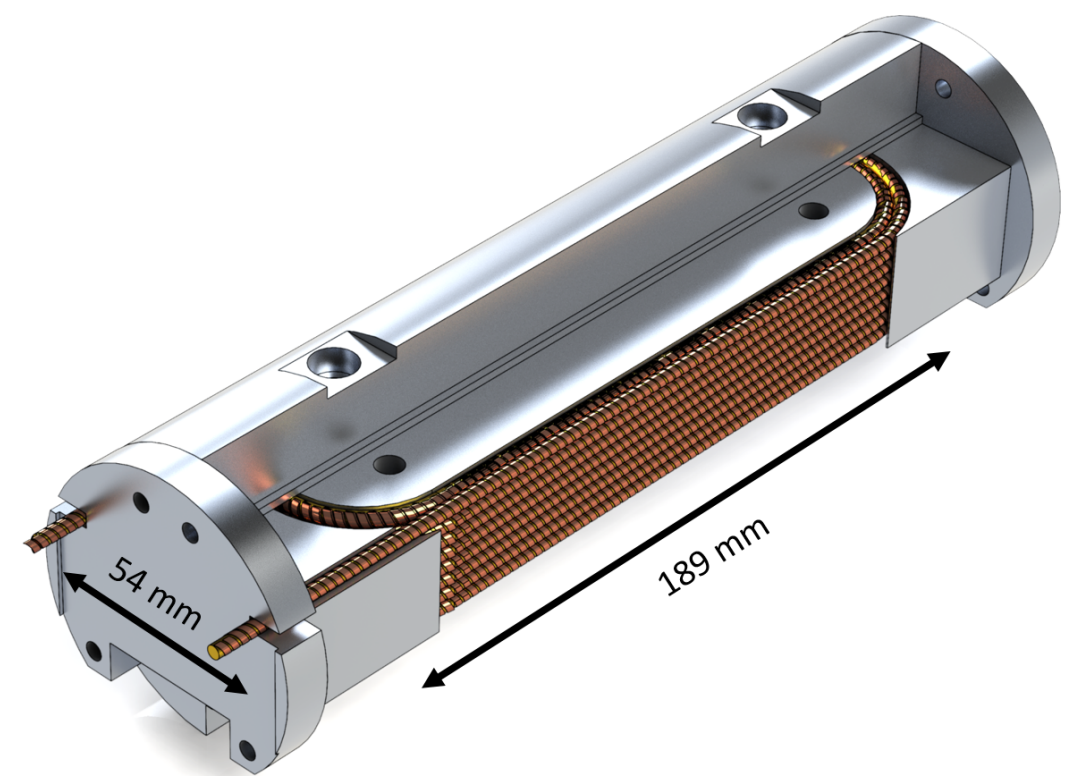

Figure 4.26: 3D render of the CORC type racetrack coil developed at CERN. The coil has 2 layers with each 8 turns of state-of-the art CORC wire. The racetrack is $189 \mathrm{~mm}$ in length and has a bore of $40 \mathrm{~mm}$ wide.

\subsubsection{CORC Wire for the Racetrack Coil}

Two CORC wires were manufactured by ACT on CERN order for the racetrack coil. Both wires are $7.96 \mathrm{~m}$ in length and included $200 \mathrm{~mm}$ long terminals on each end, shown in Figure 4.27. The first CORC wire comprised mainly stainless steel tapes, two ReBCO tapes and four VIC (Variable $\mathrm{I}_{c}$ ) tapes. VIC tapes are leftover ReBCO tapes from various tape batches, which properties are not fully guaranteed and are often of low performance. The VIC tapes were wound between the ReBCO tapes and the steel tapes to make a soft transition between the hard and also sharp stainless steel tapes and the two ReBCO tapes in the outer layer of the dummy wire. The critical current of this 'dummy' wire at $77 \mathrm{~K}$ is some $130 \mathrm{~A}$ provided by the two ReBCO tapes with known critical current and some additional critical current provided by the VIC tapes. The dummy wire was used 

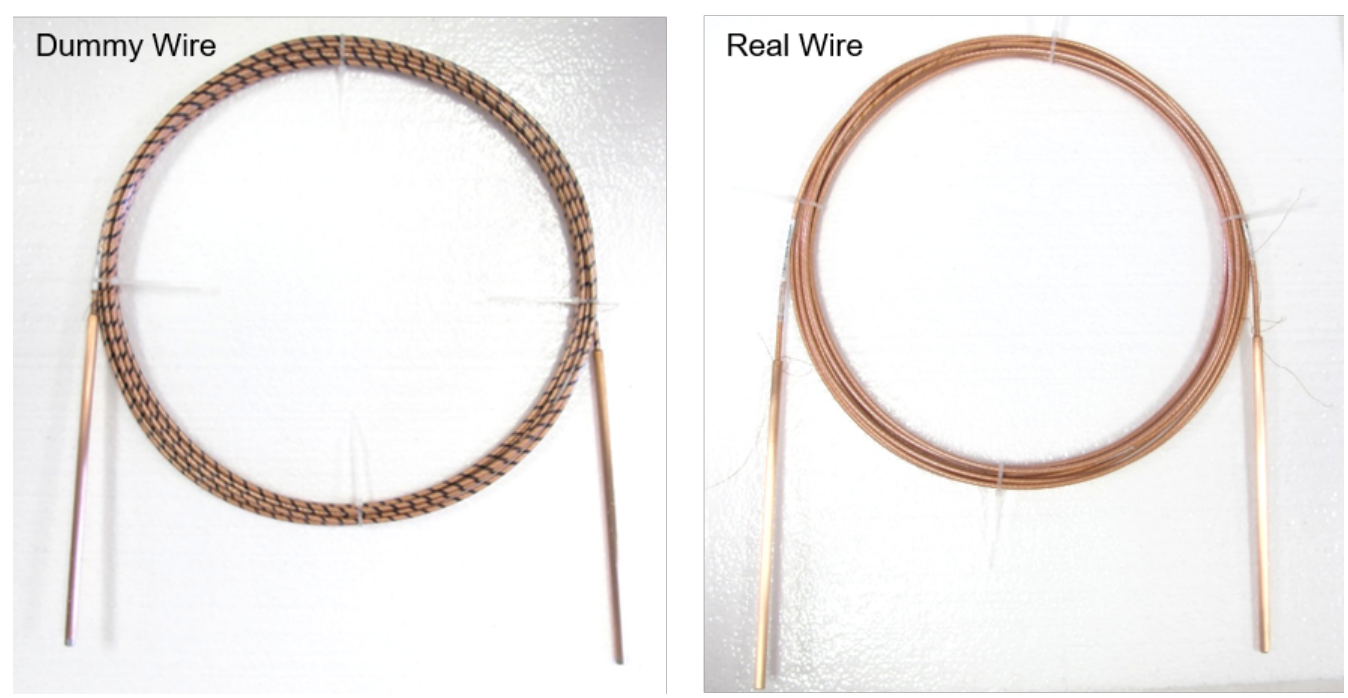

Figure 4.27: Dummy and real CORC wires for testing and preparing the CORC racetrack coil. Some tapes in the dummy CORC wire are marked with a black coating to differentiate them from the pristine superconducting tapes.

for testing the coil winding procedure and for testing the effect of thermal cycling on the performance of the coil. The second CORC wire has 27 SCS2030 ReBCO tapes wound in 11 layers, which closely resembles characterized CORC wire 4 described in Section 4.4.1. The tapes have an average critical current of $67 \mathrm{~A}$ at $77 \mathrm{~K}$. The critical current of the CORC wire is estimated at $1800 \mathrm{~A}$ at $77 \mathrm{~K}$. Details on both CORC wires are shown in Table 4.5. The real CORC wire has two layers more compared to the dummy wire as the stainless steel tapes in the dummy wire are $13 \mu \mathrm{m}$ thicker than the ReBCO tapes. The wire diameter is in both cases almost identical.

Table 4.5: Specification of the dummy and real CORC wires for the CORC type racetrack coil.

\begin{tabular}{lccc}
\hline \hline & Dummy wire & Real wire & Unit \\
\hline Number of layers & 9 & 11 & - \\
Number of ReBCO tapes & 2 & 27 & - \\
Number of VIC tapes & 4 & 0 & - \\
Number of stainless steel tapes & 16 & 0 & - \\
ReBCO tape type & SCS2030 & SCS2030 & - \\
Outer diameter & 3.40 & 3.45 & $\mathrm{~mm}$ \\
Core material & $\mathrm{Cu}$ & $\mathrm{Cu}$ & - \\
Core diameter & 2.35 & 2.35 & $\mathrm{~mm}$ \\
Predicted $\mathrm{I}_{c}(77 \mathrm{~K}$, self-field $)$ & $\sim 130$ & 1800 & $\mathrm{~A}$ \\
Predicted $\mathrm{I}_{c}(4.2 \mathrm{~K}, 10 \mathrm{~T})$ & $\sim 377$ & 5250 & $\mathrm{~A}$ \\
\hline \hline
\end{tabular}


The dummy CORC wire was characterized at $77 \mathrm{~K}$ and self-field to set its reference performance. First, it was tested in the configuration as delivered (Figure 4.31a). Copper braid current leads were soldered to the cylindrical joint terminals of the sample and voltage taps were attached. The wire was placed in a container and submerged in liquid nitrogen. The measured critical current of the wire is $235 \mathrm{~A}$ with an n-value of 10 . The peak self-field in this configuration is $0.35 \mathrm{mT} / \mathrm{A}$. After this test the wire was wound to a 12-turn solenoid to reduce the peak self-field to $0.26 \mathrm{mT} / \mathrm{A}$. The critical current increased to $257 \pm 1 \mathrm{~A}$ with the same n-value of $10.3 \pm 0.1$. The VIC tapes added $126 \mathrm{~A}$ to the expected $\mathrm{I}_{c}$ of $130 \mathrm{~A}$ of the two known ReBCO tapes for a total wire $I_{c}$ of $256 \mathrm{~A}$.
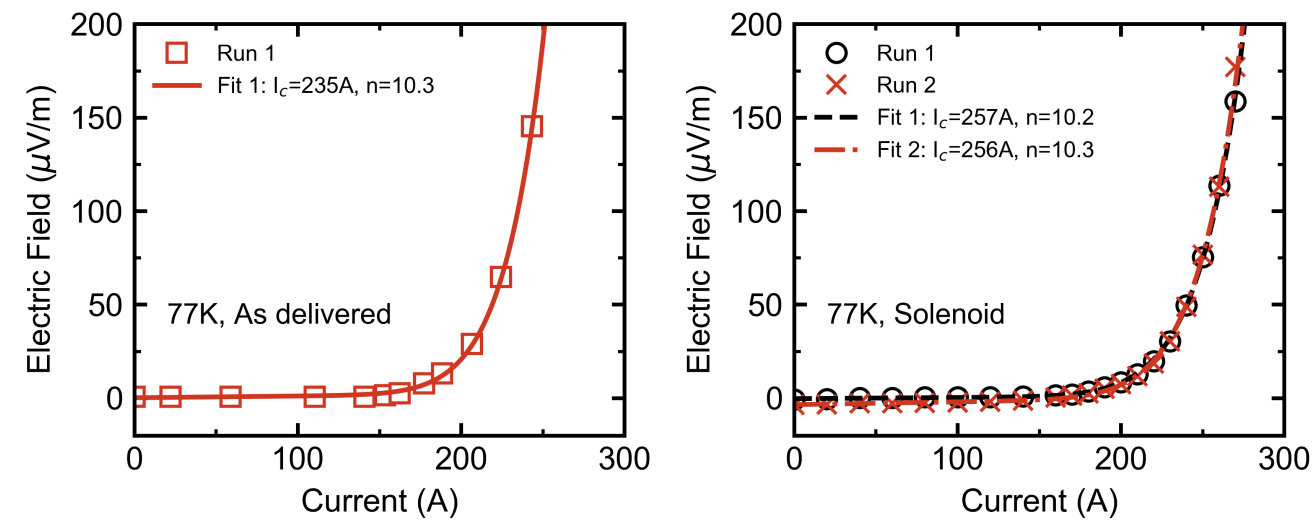

Figure 4.28: Left: electric field over the CORC wire as function of current measured in the shape as the wire was delivered (Figure 4.31a). Right: electric field over the same CORC wire wound to a 12 turn solenoid. A clear transition at around $257 \mathrm{~A}$ and an $n$ value just over 10 is observed.

\subsubsection{Winding and Test of the CORC Racetrack Demonstrator}

The wires were wound and impregnated with epoxy resin (Stycast 2580 FT). The polyethylene insulation around the CORC wire is not removed and prevents the epoxy resin flowing in-between the tapes of the CORC wire. Epoxy resin in-between tapes can lead to delamination of the tapes during cool down caused by thermal shrinkage mismatch between ReBCO tapes and epoxy resin. Such degradation in ReBCO coils was observed and described by T. Takematsu et al. in [103].

The coil has two layers of wire on a stainless steel mandrel. Winding starts with one turn in the outer layer and continues with all turns of the inner layer. Thereafter, the remaining turns of the outer layers are wound. A schematic of the winding method is shown in Figure 4.29. This winding configuration allows practical inlet and outlet positions of the CORC wire in the outer layer on one side of the magnet without complicated bends. Any voids between turns and layers are filled with epoxy resin.

The inner layer of the dummy racetrack was dry-wound to test the winding procedure before the layer is wet-wound with epoxy resin. Each turn was pushed in place before 
b

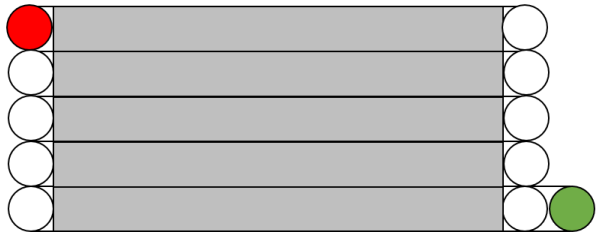

c

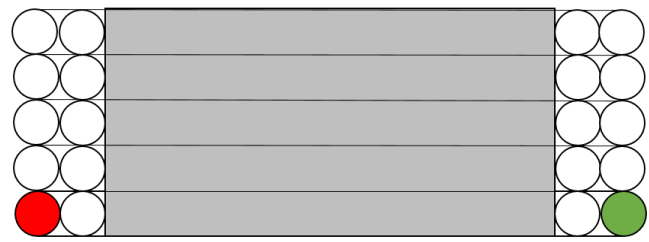

Figure 4.29: Winding schematic of the CORC type racetrack coil. Winding starts with one turn in the outer layer and continues with one turn in the inner layer (a). The entire inner layer is wound (b). The coil is finished by winding the outer layer (c). This winding method ensures a practical wire inlet and outlet position on one side of the racetrack coil, thereby avoiding any complicated bends to extract the wire from the coil.

adding a new turn. The CORC wire deformed slightly to a more oval shape in the bends at each side of the racetrack. This deformation was expected and included in the margin. The outer turn was wound once the epoxy resin of the inner turn completely dried. The outer layer was wet-wound and proved more difficult to wind. Some layers had to be forcefully put in place because otherwise they would immediately spring out of the winding pack.

Winding the dummy CORC wire proved more difficult than expected and several complications arose [102]. The polyethylene insulation ruptured during winding, likely due to tight bending of the CORC wire. The ruptured sleeve was repaired with polyimide thin tape to prevent the epoxy resin to enter the insulation sleeve. Although the CORC wire should be able to handle the $40 \mathrm{~mm}$ winding diameter, the insulation did not. The CORC wire was more springy than expected and, in combination with the layer of epoxy resin, difficult to put in place by force.

Pictures of the winding process are presented in Figure 4.31. Given the experience, it is likely that some degradation was introduced, later confirmed by the test of the coil.

The coil was tested in a liquid nitrogen bath. The current leads were soldered to the cylindrical joint terminals and voltage taps were placed on both wire ends. Three $\mathrm{I}_{c}$ measurements were conducted with thermal cycles to room temperature in between. The racetrack coil was cooled down slowly with liquid nitrogen to prevent any shrinkage related issues. A critical current of $24 \mathrm{~A}$ was measured with a coil n-value of 4.4, which is an $\mathrm{I}_{c}$ retention of only $10 \%$ as shown in Figure 4.30. The wire showed severe degradation apparently introduced by winding it to the racetrack coil shape. The racetrack coil was thermally cycled twice and the critical current increased slightly to 30 A with a coil nvalue of 4.1 in the third measurement run. The average measured critical current of the three measurements is $27 \pm 3$ A with a n-value of $4.2 \pm 0.2$. The linear component in the superconducting transition has a constant resistance of $5.5 \pm 0.2 \mu \Omega$, which is likely caused 

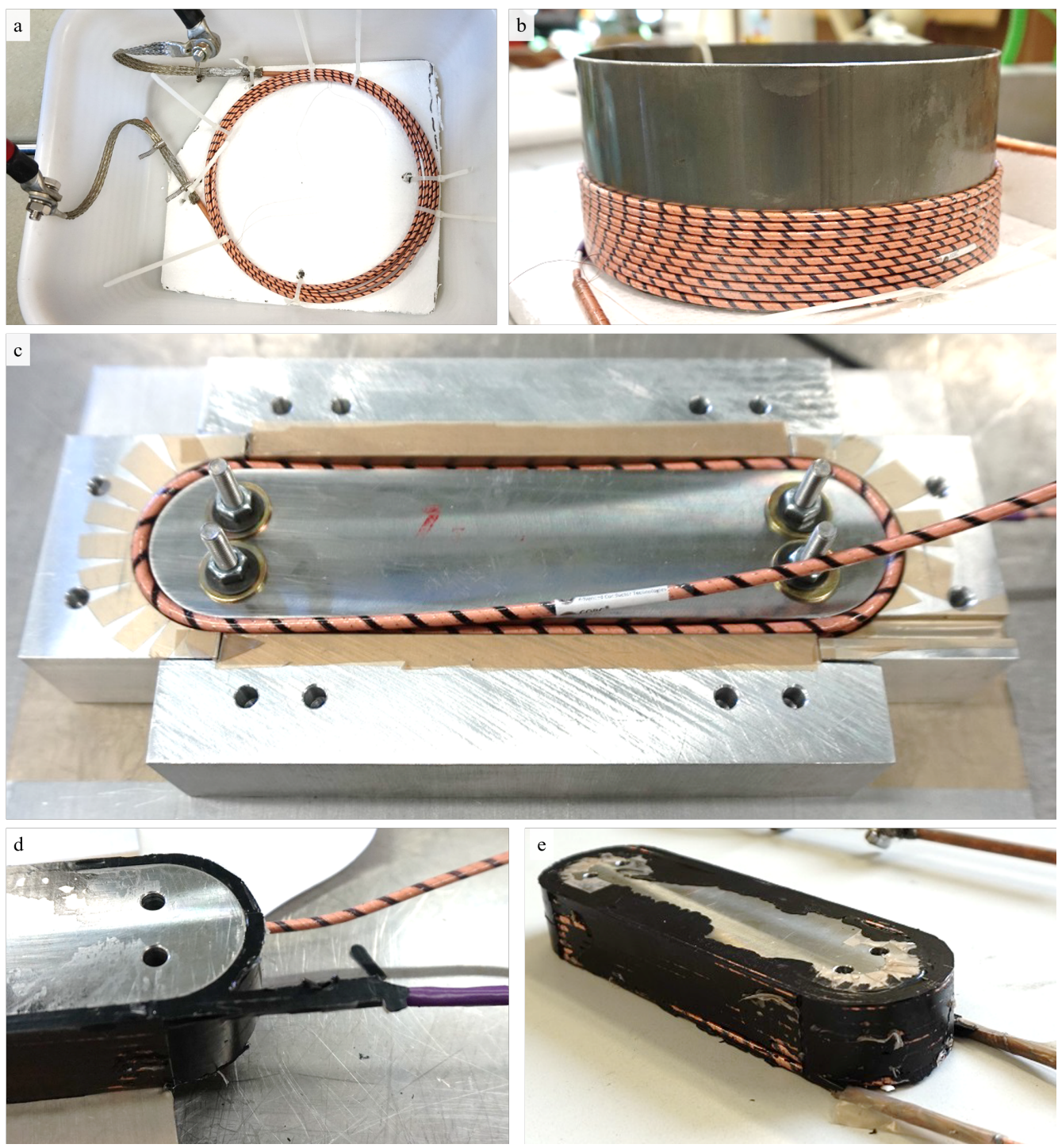

Figure 4.31: Pictures of preparation and testing of the first trial CORC racetrack coil using the dummy wire [102]. Picture (a) shows the test configuration of the dummy CORC wire as it was delivered. Picture (b) shows the 12-turn solenoid configuration for the subsequent test. Picture (c) displays winding of the final turn of the inner layer before adding epoxy resin. Pictures (d) and (e) show the wet-wound inner layer of the coil and the full racetrack coil. 
by current redistribution in the heavily degraded sections. Following this experience, the CORC wire production parameters need to be adjusted to avoid the degradation observed. The next winding trial is beyond the scope of this work.

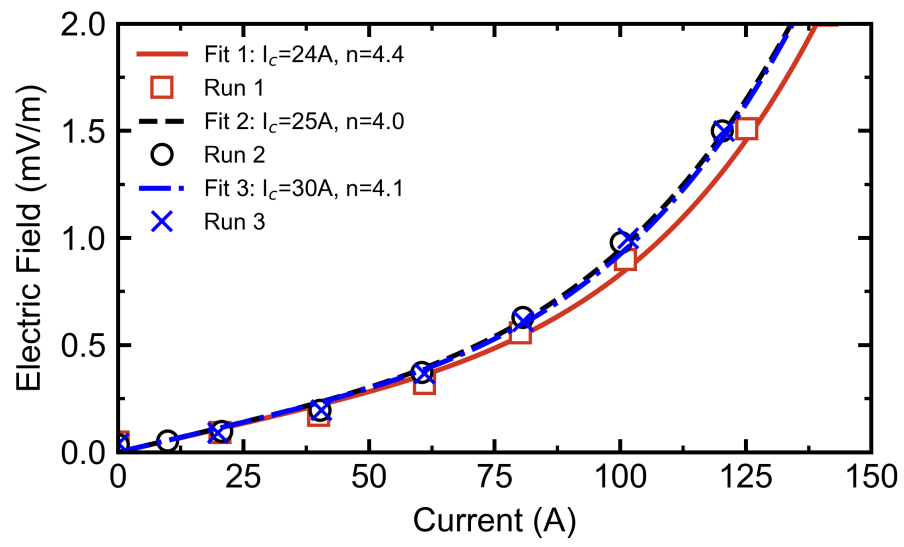

Figure 4.30: Average electric field over the entire CORC racetrack coil as function of current at $77 \mathrm{~K}$ for three subsequent measurement runs with thermal cycling to room temperature in between. Severe degradation is observed as the critical current is reduced from $257 \pm 1 \mathrm{~A}$ before winding to $27 \pm 3 \mathrm{~A}$ after winding.

\subsection{Conclusion}

A CORC cable with 38 ReBCO SCS4050 tapes was characterized at 4.2 and $1.9 \mathrm{~K}$ in a magnetic field up to $9.6 \mathrm{~T}$. The CORC sample is a $1.8 \mathrm{~m}$ section out of a $12.2 \mathrm{~m}$ long CORC cable prepared for use in the world's first CORC six-around-one Cable-In-Conduit Conductor. The CORC sample has a benchmark critical current of $7.9 \mathrm{kA}$ at $4.2 \mathrm{~K}$ and $10 \mathrm{~T}$. A reduction of temperature from $4.2 \mathrm{~K}$ to $1.9 \mathrm{~K}$ increases the critical current of the CORC conductor by $5 \%$. The measured critical current is very close to the expected critical current, demonstrating the validity of the method for predicting CORC conductor performance. The CORC cable, while shunted with a low-resistance shunt, was quenched several times during the test either with quench heaters or by pushing current over the conductor's critical current. The sample survived all quenches and demonstrated the superb stability of HTS conductors compared to LTS conductors.

Four state-of-the-art CORC wires were developed and characterized at the University of Twente. The wires are thinner, more flexible and have higher current densities than the CORC cable variants. The first CORC sample, comprising $3 \mathrm{~mm}$ wide ReBCO tapes, showed severe degradation due to winding the wire around the $60 \mathrm{~mm}$ diameter bore of the sample holder, which proves that the more narrow $2 \mathrm{~mm}$ wide ReBCO tape is a necessity for degradation free bending of CORC wires on a $60 \mathrm{~mm}$ diameter. The two 
latest CORC wires, 3 and 4, show critical current densities of 322 and $412 \mathrm{~A} / \mathrm{mm}^{2}$ at $4.2 \mathrm{~K}$ and $10 \mathrm{~T}$ with n-values of $30 \pm 15$ and $32 \pm 7$, respectively. The critical current density is lower than expected for both CORC wires. Single tapes from both wires were extracted and analyzed at ACT to pinpoint and quantify any degradation. An $\mathrm{I}_{c}$ retention of $48 \%$ was found for CORC wire 3 and $82 \%$ for CORC wire 4 . The results are used as feedback to optimize and improve future CORC wire production and handling.

$R e \mathrm{BCO}$ coated conductors with the ReBCO layer positioned closer to the neutral axis by either reducing substrate thickness or by copper plating only one side of the tape could further reduce the diameter of the CORC wire, thereby increasing its flexibility and current density. CORC wires comprising ReBCO tapes with a centered ReBCO layer are currently under development [93] and can boost CORC based magnet technology.

Development of ReBCO type coils using either a single ReBCO coated conductor or multistrand $\mathrm{Re} \mathrm{BCO}$ conductors is ongoing and has taking huge leaps forwards in recent years. A CORC type racetrack coil is under development at CERN for demonstrating the feasibility of CORC wires in high-field magnets. A first trial racetrack coil was prepared using 'dummy' CORC wire to test the winding procedure and the effect of thermal cycling on the performance. Several points for improvement were identified. 


\section{Chapter 5}

\section{Six-Around-One CORC Cable-In-Conduit Conductors}

The ReBCO CORC Cable-In-Conduit Conductor is a high-current multi-strand conductor aimed at application in large-scale magnets for detector-and fusion experiments, but also for the bus lines that feed high currents to such magnets. ReBCO-based conductors open the operating temperature range of 30 to $50 \mathrm{~K}$ and thus allow for a significant reduction in cooling cost and a simplification of the refrigeration plant. Such conductor also makes it feasible to design magnets capable of producing a magnetic field far beyond $20 \mathrm{~T}$ at $5 \mathrm{~K}$ and offers a significant increase in thermal stability compared to niobium-based conductors. In this chapter the design, preparation methods and testing of three CORC six-around-one Cable-In-Conduit Conductors are presented.

\subsection{Introduction}

The concept of the Cable-In-Conduit Conductor (CICC) is already over 50 years old. Early adaptation of the concept showed superconducting wires soldered around a helium filled tube. Such conductor can be actively cooled without a bath of liquid helium or without relying on conduction cooling through the entire winding pack of a magnet. The Morpurgo dipole [104] and the Vertex split solenoid magnets at CERN [105], both built in the 1970s, were pioneering magnets by incorporating Cable-In-Conduit Conductors and are still operational today. A historical picture of the conductor used in the Morpurgo magnet is presented in Figure 5.1a. The next generation of CICCs encapsulates superconducting strands in direct contact with the helium to increase the contact surface between superconductor and cryogen, thereby improving heat transfer and stability of the conductor. This is the modern day concept of the Cable-In-Conduit Conductor. CIC-type conductors have two main advantages compared co-extruded or other multi-strand conductors: 
(a)

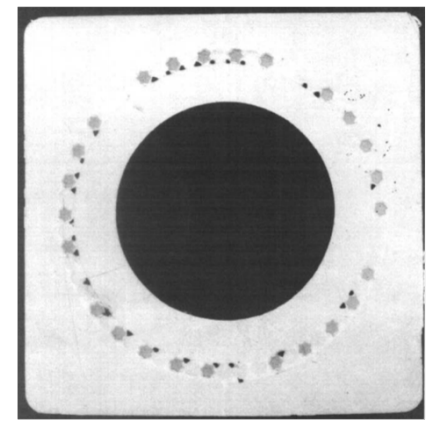

(b)

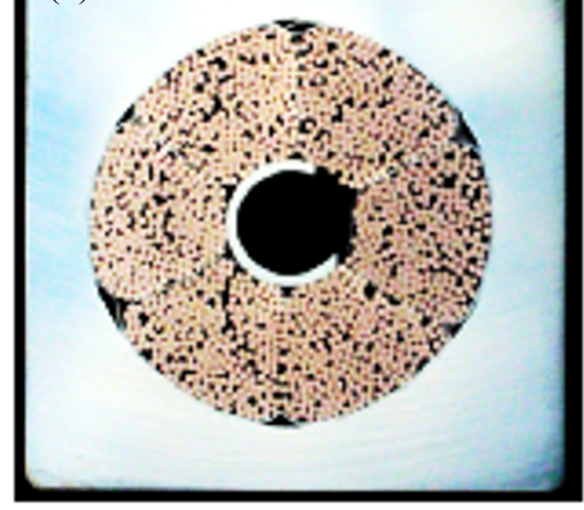

Figure 5.1: Picture (a) shows the square composite conductor used in the Morpurgo dipole magnet at CERN in the 1970s [104]. This conductor comprises many NbTi/Cu strands sandwiched and soldered between two copper profiles with a cooling channel in its center. Picture (b) shows a modern CICC used for the ITER central solenoid displaying the six-around-one structure of the strand petals [106].

- the thermal stability of cable-in-conduit conductors is very high due to direct cooling of the superconducting strands with either forced-flow gas or super-critical liquid helium;

- the accumulating Lorentz forces in multi-layer coils is taken up by the conduit, therefore the superconducting strands only suffer from the local Lorentz force.

Recent examples of well-known CICCs are the $\mathrm{NbTi}$ and $\mathrm{Nb}_{3}$ Sn conductors for magnets in the ITER experiment. Fusion experiments, like ITER, require magnets with large volumes to enclose the fusion plasma and a high magnetic field to contain and to shape the fusion process. The conductors have to endure significant electro-magnetic and heat loads when the magnets are powered and plasma is present in the fusion-chamber. At the time of development of the ITER experiment, it was only possible to reach these demanding specifications with $\mathrm{NbTi}$ and $\mathrm{Nb}_{3} \mathrm{Sn}$ superconducting strands, directly cooled by supercritical helium inside a conduit. A picture of one of the ITER Central Solenoid conductor is presented in Figure 5.1. Although such conductor is able to reach impressive currents of up to $50 \mathrm{kA}$ at $13 \mathrm{~T}$ and $4.5 \mathrm{~K}$, several issues arose during the production and testing of these $\mathrm{Nb}_{3} \mathrm{Sn}$ conductors. $\mathrm{Nb}_{3} \mathrm{Sn}$ cables and strands are notorious for their degradation due to local stress concentrations. One of the main challenges of preparing CICCs using this material is handling the considerable electro-magnetic loads during operation, combined with the loads due to thermal shrinkage during cool-down from room temperature to $4.5 \mathrm{~K}$. Recent studies share a pessimistic view on the long term survival of some of the ITER conductors [107].

Another drawback of $\mathrm{Nb}_{3} \mathrm{Sn}$ conductors is that they require extensive heat treatment after coil winding. Heat treatment of small samples and coils for laboratory environments is readily realized with commercial furnaces. However, heat treatment of large coils reaching 
several meters in size is not straight forward and requires dedicated furnaces designed specifically for such coils. The possibility of replacing $\mathrm{Nb}_{3} \mathrm{Sn}$ with a material that does not require a heat treatment would highly simplify the production process of large magnets with a design magnetic field exceeding $10 \mathrm{~T}$.

ReBCO-based CICCs may be a solution for future fusion experiments and other large magnet systems. Recent developments on ReBCO coated conductor technology have provided a significant boost to their electrical performance. The ReBCO-based conductors are able to surpass $\mathrm{NbTi}$ and $\mathrm{Nb}_{3} \mathrm{Sn}$ conductors in terms of current and current density at $4.5 \mathrm{~K}$ in magnetic fields exceeding $15 \mathrm{~T}$. ReBCO tapes do not require heat treatment after winding, which is a significant advantage for large magnets systems. Even more importantly, $R e \mathrm{BCO}$ opens the operating temperature range of 30 to $50 \mathrm{~K}$, which drastically simplifies the cryogenic system and reduces the operating expenses as the conductors can be fully gas cooled.

\section{ReBCO Based Cable-In-Conduit Conductors}

Two ReBCO conductors have been pursued to be used as strands for HTS CICCs; the Twisted Stacked Tape Conductor (TSTC) conductor [21] and the CORC conductor [108]. The TSTC conductor has a relatively large minimum bending radius, which limits its potential for compact high-field magnets, and is difficult to twist due to its tape layout. However, in large magnets, with bore diameters of several meters, the minimum bending diameter of the TSTC conductor is not an issue. The first demonstration ReBCO CICC using TSTC stands was presented by Celentano et al. [38]. This CICC comprises 5 strands spiraled within an aluminium slotted core and is designed to reach $20 \mathrm{kA}$ at $4.2 \mathrm{~K}$ and $15 \mathrm{~T}$. Another variant of the TSTC CICC is pursued by the group of Bruzzone at the Swiss Plasma Center, as decribed by Uglietti et al. [109, 39]. In this case, the TSTC stand is encapsulated by two copper half-shells to provide a round cable shape. The round strand is then wound around a former to create a Rutherford-type conductor rated for $60 \mathrm{kA}$ at $5 \mathrm{~K}$ and $12 \mathrm{~T}$. Both conductors were developed for application in fusion-type magnets. Pictures of both TSTC CICCs are presented in Figure 5.2.

Development of the ReBCO CORC CICC is performed by CERN in a common effort with Advanced Conductor Technologies. CERN has an interest in the CORC CICC for feeding high currents over long lengths to large detector magnets and potentially, at a later stage, implement them as the main conductor in a detector magnet. The CORC CICC offers a high current carrying capability and high thermal- and electrical stability in combination with straightforward cabling of the CORC strands. These are all prerequisites for conductors aimed at detector magnets and bus lines.

In this chapter the developments of such CORC CICC is described including design, preparation and testing of three first-in-the-world CORC CIC-Conductors, followed by a conceptual design of a CORC bus bar designed to feed current to future detector magnets. 


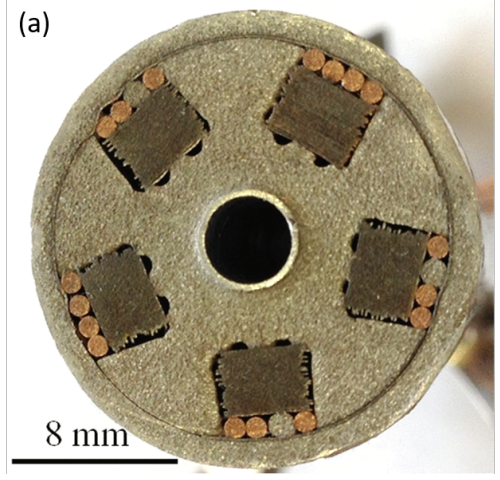

(b)

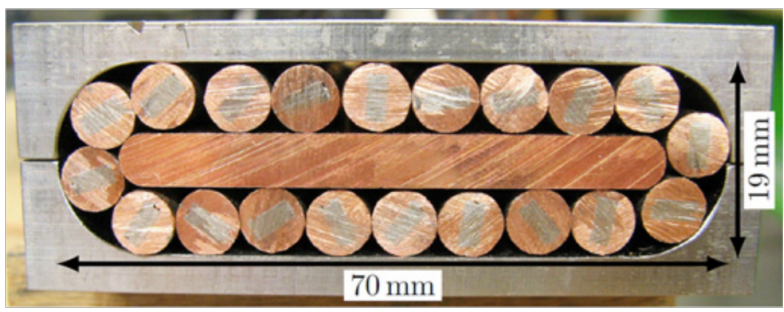

Figure 5.2: (a) Picture of the demonstration TSTC CICC from Celentano et al. [38] and (b) picture of the TSTC CICC from Uglietti et al. [39] .

\subsection{Design of CORC type Cable-In-Conduit Conductors}

The round shape of the CORC conductor supports a variety of CICC layouts. The most straightforward one is the 'six-around-one' layout, presented in Figure 5.3. This layout is pursued at CERN.

\subsubsection{CORC CICC Layout}

The design of the CORC CICC has six CORC strands helically wound around a metal rod or hollow tube. CORC strands are relatively flexible and allow straightforward bundling. The six-around-one cable is enclosed by a square, round or rectangular jacket. The jacket is ideally manufactured from a high yield strength aluminium alloy, which is lightweight, inexpensive and strong, while maintaining good electrical and thermal properties. A jacket manufactured from copper or aluminium alloy allows conduction cooling. However, due to its high electrical conductivity, a copper jacket is prone to higher AC-loss in variable magnetic fields and most importantly, the yield strength of OFHC copper is relatively low and therefore cannot sustain large electro-magnetic loads. A stainless steel jacket has a considerably higher yield strength and has low eddy current loss due to its higher electrical resistivity. The downside of steel is that it offers no significant contribution to

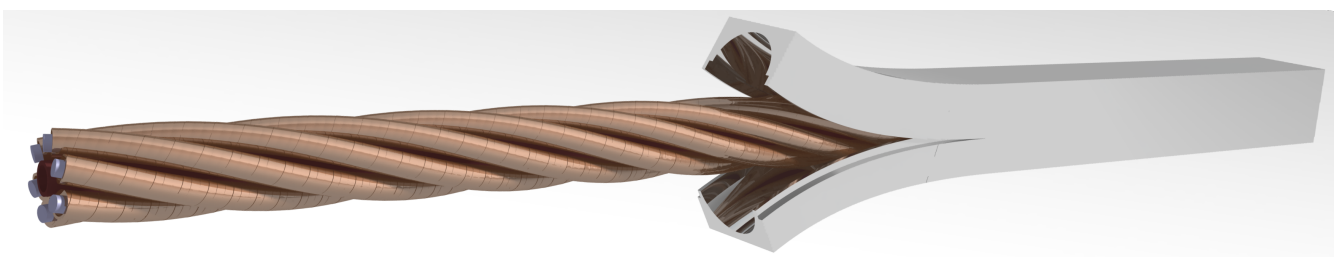

Figure 5.3: Conceptual image of a CORC-type six-around-one Cable-In-Conduit Conductor. 
thermal- and electrical stability of the conductor, nor to quench protection. Therefore, additional copper needs to be added internally to increase the copper-to-superconductor ratio of the cable.

Internal helium flow of either gas or super-critical liquid can be established via parallel flow in a central perforated tube and the voids between strands. Direct contact between strands and cryogen is beneficial for the stability of the conductor and thus preferred, especially under high ramp-rate conditions. In DC-operated magnets, i.e. large detector magnets, it is more practical to apply a conduction-cooled conductor. In this case, the central tube can be exchanged for a rod and all voids between strands can be filled with epoxy or solder. Solder increases the heat capacity of the conductor and reduces the thermal resistance to the nearest cooling channel. The thermal contraction of aluminium is larger compared to that of common soldering materials. Therefore, if the conductor is cooled to its operating temperature, a tight fit between aluminium jacket and cable is assured.

\section{Other variations of CORC Cable-In-Conduit Conductors}

Other variations of the CORC Cable-In-Conduit Conductor layout can be envisioned. One example is the CORC triplet: three twisted CORC strands in a rectangular or circular jacket. The three-strand cable is thinner and a bit easier to handle than the six-around-one cable variant.

Another variation utilizes many thin CORC strands, each comprising only a few ReBCO layers, positioned around a core with a relatively large diameter. The advantage of such design is a high contact surface between tapes and cold gas, thereby providing a large thermal stability. The development of thin CORC wires enables such CORC CICC design. Schematic cross-sectional representations of three CORC CICC variants are shown in Figure 5.4 .

\subsubsection{Realization of an Exercise Model CORC CICC}

A trial CORC CICC is assembled using dummy CORC strand, comprising copper and stainless steel tapes, to exercise the cabling, jacket mounting and welding steps. The six strands are twisted manually with a pitch length of approximately $400 \mathrm{~mm}$. The conduit has outer dimensions of $30 \times 30 \mathrm{~mm}^{2}$ and comes in two halves closed by Electron Beam (EB) welding. Both halves interlock due to a step-shaped groove on their edges. This shape also prevents the welding arc to break through the conduit and possibly damage the cable during welding. Long lengths can be welded following this method. Aluminum 5083 is used in the first production runs because of its proven performance for EB-welding. Three practice runs were carried out on empty jackets to test the welding procedure and to monitor the temperature on the inner surface of the bore of the jacket during welding. This temperature in and on the jacket did not exceed $160^{\circ} \mathrm{C}$ and therefore is unlikely to damage the CORC strands. The weld between the two halves is thin, as illustrated in Figure 5.5. All assembly steps proved successfully. 

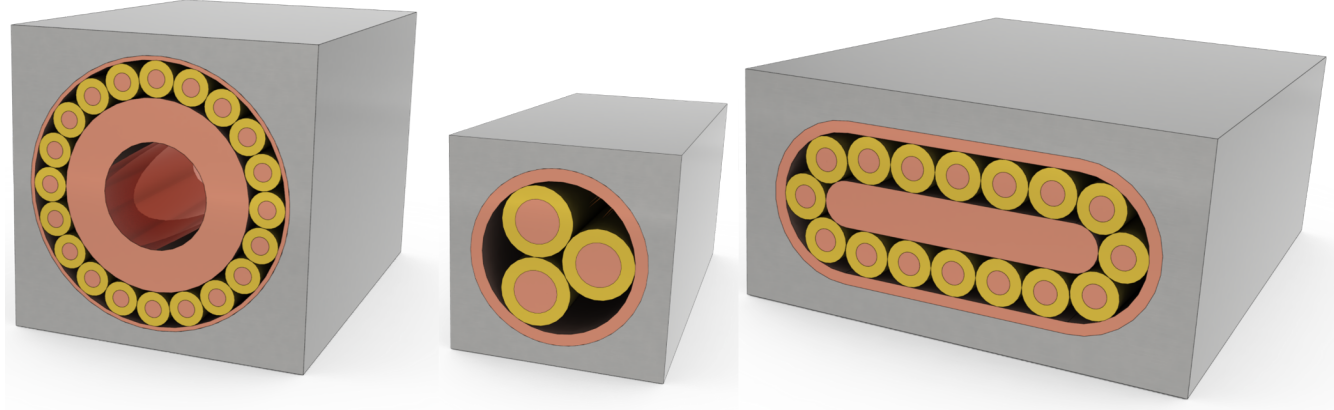

Figure 5.4: Three variations of CORC CICCs with on the left a CICC design comprising many thin CORC wires. This design has a high thermal and electrical stability, but has a relatively low current density due to its large bore. The middle variation is the CORC triplet. The cable current density is slightly lower than that of the six-around-one variant, because of the large void fraction. The right layout is similar to the conductor layout presented by Uglietti et al. in [39] for their TSTC conductor. This CICC-layout is very suitable for the CORC type conductors due to its natural round shape.

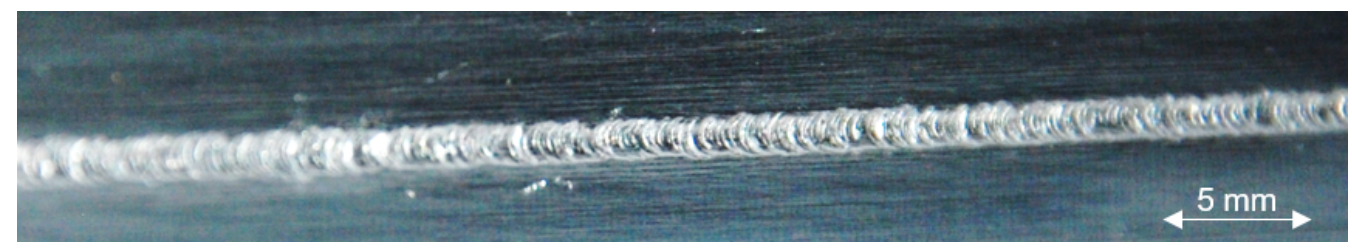

Figure 5.5: Closeup of the weld produced with electron-beam welding between two 5083 series aluminium alloy halves of the jacket.

\subsection{Worlds First CORC type Cable-In-Conduit Conductor}

A $1.7 \mathrm{~m}$ long CORC CICC is designed, prepared and tested at CERN to demonstrate its feasibility and large current carrying capability. This conductor is the first of its kind and has a design current of $47 \mathrm{kA}$ at $4.2 \mathrm{~K}$ and $10 \mathrm{~T}$. The CICC has been tested at CERN at a temperature of $4.2 \mathrm{~K}$ in an external magnetic field of up to $9 \mathrm{~T}$ and at $77 \mathrm{~K}$ in self-field. Pictures of the cross-section of a short CORC CICC sample and of its strand are presented in Figure 5.7.

\subsubsection{Design and Properties}

The CORC cable bundle comprises six CORC strands of each 38 SuperPower SCS4050 tapes, for a total of 228 tapes. The tapes are $4 \mathrm{~mm}$ wide, $96 \mu \mathrm{m}$ thick and each have a critical current of $130 \mathrm{~A}$ at $77 \mathrm{~K}$ and self-field. The strands contain an aluminum core 

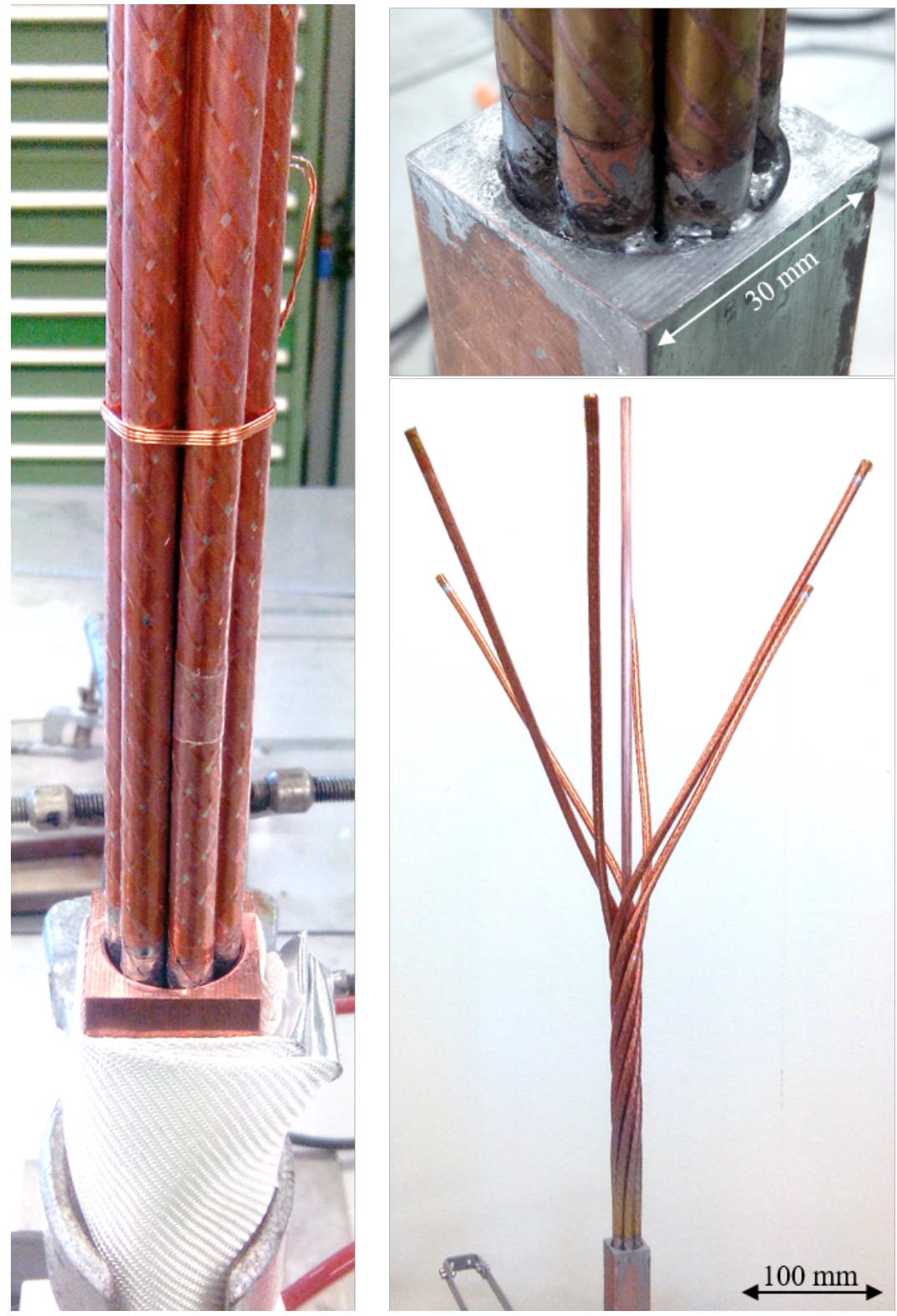

Figure 5.6: The trial model CORC CICC during preparation. The photo on the left shows the cable bundle inserted in the bore of the joint terminal before it is filled with solder. The photo on the top right shows the solder-filled terminal with the CORC cable bundle. After filling the joint terminal, the strands are manually twisted to a pitch of approximately $400 \mathrm{~mm}$ as demonstrated in the photo on the bottom right. 


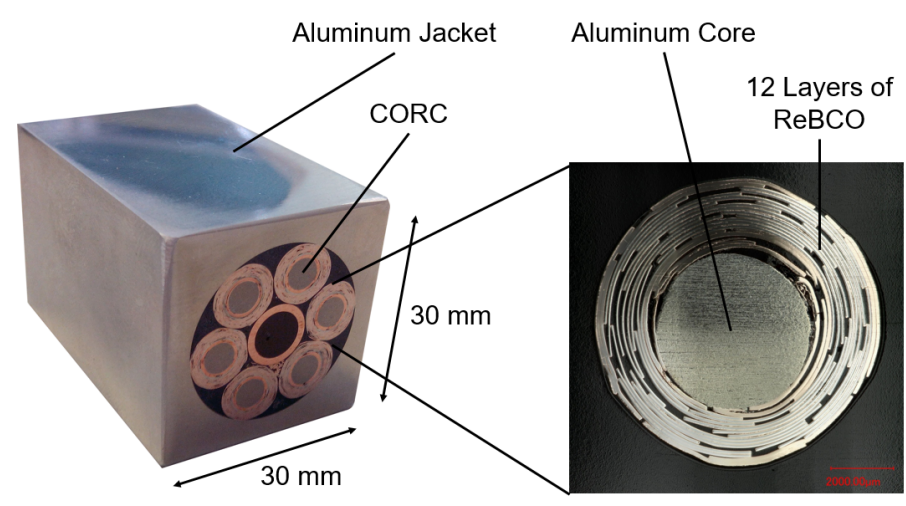

Figure 5.7: Cross-section of a model version of the first CORC CICC and one of its strands.

with an outer diameter of $4 \mathrm{~mm}$. Several layers of copper tapes increase the core diameter to $5 \mathrm{~mm}$, followed by 12 layers of ReBCO tapes and finished with 2 additional layers of copper tapes for mechanical protection. The outer diameter of the strand is $7.6 \mathrm{~mm}$. A single strand was characterized in the FRESCA facility at CERN, as described in Section 4.3. The measured critical current of one strand is $7.9 \mathrm{kA}$ at $4.2 \mathrm{~K}$ and $10 \mathrm{~T}$, which corresponds to a overall strand current density of $174 \mathrm{~A} / \mathrm{mm}^{2}$.

The cable bundle is $1500 \mathrm{~mm}$ long with the six CORC strands helically wound around a central copper tube. The central tube has an outer diameter of $8 \mathrm{~mm}$, for a total cable diameter $23.2 \mathrm{~mm}$. The diameter of the bundle is increased to $24 \mathrm{~mm}$ with several layers of copper foil. The jacket comprises two halves of $\mathrm{Al} \mathrm{5083,} \mathrm{similar} \mathrm{to} \mathrm{those} \mathrm{of} \mathrm{the} \mathrm{exercise}$ model CICC and have outer dimensions of $35 \mathrm{~mm}$ by $45 \mathrm{~mm}$. These dimensions are slightly larger than the ones mentioned in Table 5.1 in order to realize a better fit with the sample holder. The voids within the main section of the CICC are left empty to facilitate space for instrumentation and wiring. The main section of the CICC has a length of $800 \mathrm{~mm}$ with at either end a joint terminal of $450 \mathrm{~mm}$ long. The joint terminals have the same outer dimensions as the conduit. The cable bundle is inserted over a length of $350 \mathrm{~mm}$ into the terminals and both are filled with solder. The predicted resistance of a single joint terminal is $2 \mathrm{n} \Omega$ at $4.2 \mathrm{~K}$ in stray field of $2 \mathrm{~T}$ and is $6 \mathrm{n} \Omega$ at $77 \mathrm{~K}$ in self-field. The rise in resistance by going from $4 \mathrm{~K}$ to $77 \mathrm{~K}$ is mainly caused by the increase in copper resistivity as function of temperature.

\subsubsection{Preparation of the CICC and the Sample Holder}

The CICC is assembled using the same procedures as used for the short trial model CICC. Photographs of the CICC in several stages of its preparation are presented in Figure 5.8. Voltage taps were attached to each CORC strand; one near each joint terminal and two at either side of the high-field region of the test facility. Thus, the voltage over each individual strand was monitored, which provided an indication of current distribution between 
Table 5.1: Properties of the world's first CORC CIC-Conductor.

\begin{tabular}{lcc}
\hline \hline Property & Value & Unit \\
\hline Number of CORC strands & 6 & - \\
Twist pitch & 0.40 & $\mathrm{~m}$ \\
Expected critical current $(4.2 \mathrm{~K}, 10 \mathrm{~T})$ & 47 & $\mathrm{kA}$ \\
Expected critical current (77 K, self-field) & 13 & $\mathrm{kA}$ \\
Joint terminal resistance (4.2 K, 2 T) & 2 & $\mathrm{n} \Omega$ \\
Joint terminal resistance (77 K, self-field) & 6 & $\mathrm{n} \Omega$ \\
Overall critical current density (4.2 K, 10 T) & 53 & $\mathrm{~A} / \mathrm{mm}^{2}$ \\
Cable critical current density (4.2 K, 10 T) & 105 & $\mathrm{~A} / \mathrm{mm}^{2}$ \\
Jacket outer dimension & $30 \mathrm{x} 30$ & $\mathrm{~mm} \mathrm{x} \mathrm{mm}$ \\
Jacket bore diameter & 24 & $\mathrm{~mm}$ \\
Central rod diameter & 8.0 & $\mathrm{~mm}^{2}$ \\
CORC strand diameter & 7.6 & $\mathrm{~mm}^{2}$ \\
Total cross-sectional area & 900 & $\mathrm{~mm}$ \\
Aluminum cross-sectional area & 523 & $\mathrm{~mm}^{2}$ \\
Copper cross-sectional area & 168 & $\mathrm{~mm}^{2}$ \\
Void cross-sectional area & 134 & $\mathrm{~mm}^{2}$ \\
\hline \hline
\end{tabular}

strands. The jacket contains several holes that serve as feed-through for the instrumentation wires and serve as inlet and outlet for liquid helium or liquid nitrogen.

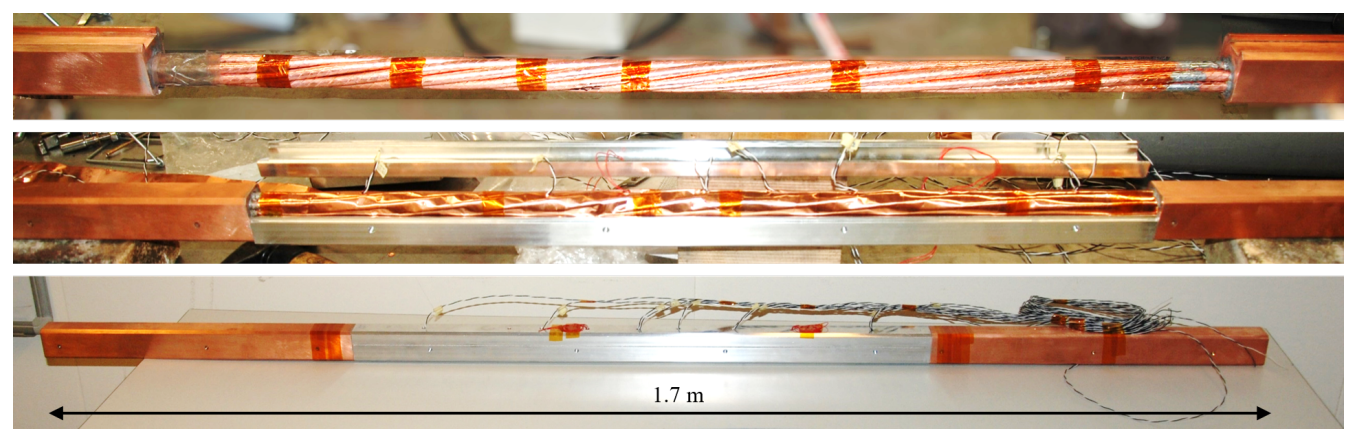

Figure 5.8: Three stages of preparation of the CICC. The top picture shows the twisted CORC cable bundle connected to the two joint terminals. In the second picture, instrumentation is attached to each CORC strand and the cable bundle is wrapped in copper foil in order to increase the bundle diameter to match the bore diameter of the jacket. The last picture shows the finished sample, with the two halves of the jacket welded together. 

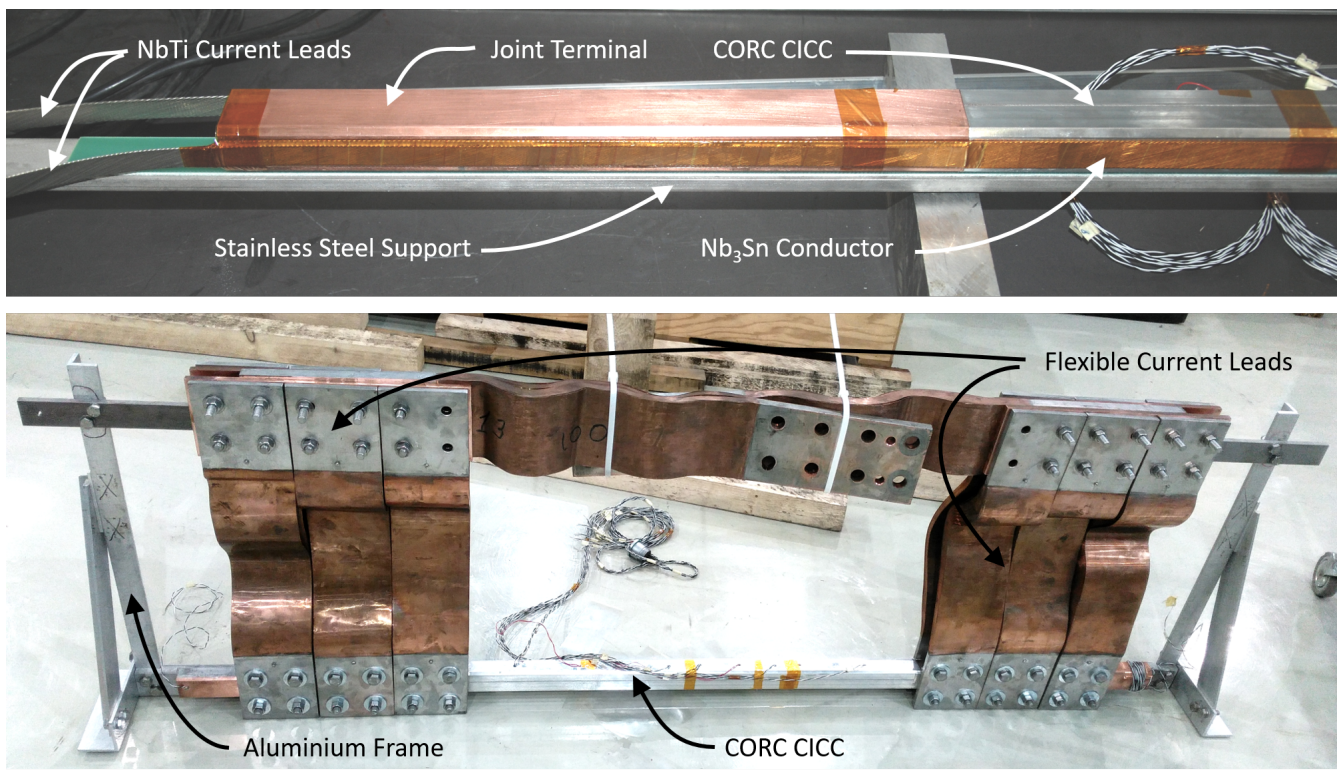

Figure 5.9: The top picture shows part of the CICC and the sample holder used for the test at $4.2 \mathrm{~K}$. The sample is bolted to a stainless steel sample holder with a layer of G10 insulation in between. The CICC is connected to the current leads of the test facility with LHC NbTi Rutherford type cables. The current flows through the CORC CICC over the high-field region and returns via a stack of $\mathrm{Nb}_{3}$ Sn conductors. The bottom picture shows the conductor and sample holder used for the test at $77 \mathrm{~K}$. The entire setup is placed in a stainless steel container filled with liquid nitrogen and is connected to a $20 \mathrm{kA}$ power supply of the facility using thick flexible copper current leads.

\section{Setups for the $4.2 \mathrm{~K}$ and $77 \mathrm{~K}$ Measurements}

The CICC was tested at $4.2 \mathrm{~K}$ in the FRESCA cable test facility at CERN. FRESCA features a 10 T dipole magnet capable of producing a homogeneous magnetic field over a length of $600 \mathrm{~mm}$ along the sample. The sample holder is designed for a hairpin-style characterization of the CORC CICC. The layout of the sample holder is similar to the one used for characterizing single CORC strand (see Section 4.3). The holder comprises a 'go' and a 'return' leg. The CICC itself is used as go-leg to transport the current through the peak-field region. A stack of three $\mathrm{Nb}_{3} \mathrm{Sn}$ Rutherford cables serves are return-leg. The sample is cooled in a bath of liquid helium at $4.2 \mathrm{~K}$. The peak current the facility can provide is $32 \mathrm{kA}$, which is lower than the expected conductor critical current of $47 \mathrm{kA}$ at $10 \mathrm{~T}$.

The CORC CICC was also tested at a temperature of $77 \mathrm{~K}$ in self-field at the SM18 magnet test facility at CERN. The CICC was placed horizontally in a stainless steel container filled with liquid nitrogen. Current is injected in two sides of the joint terminals using massive flexible copper current leads. The test facility provides $20 \mathrm{kA}$, sufficient to reach the conductor's critical current at $77 \mathrm{~K}$ in self-field. Pictures of both sample holders are presented in Figure 5.9 and a schematic of the joint cross-sections is shown in Figure 5.10. 

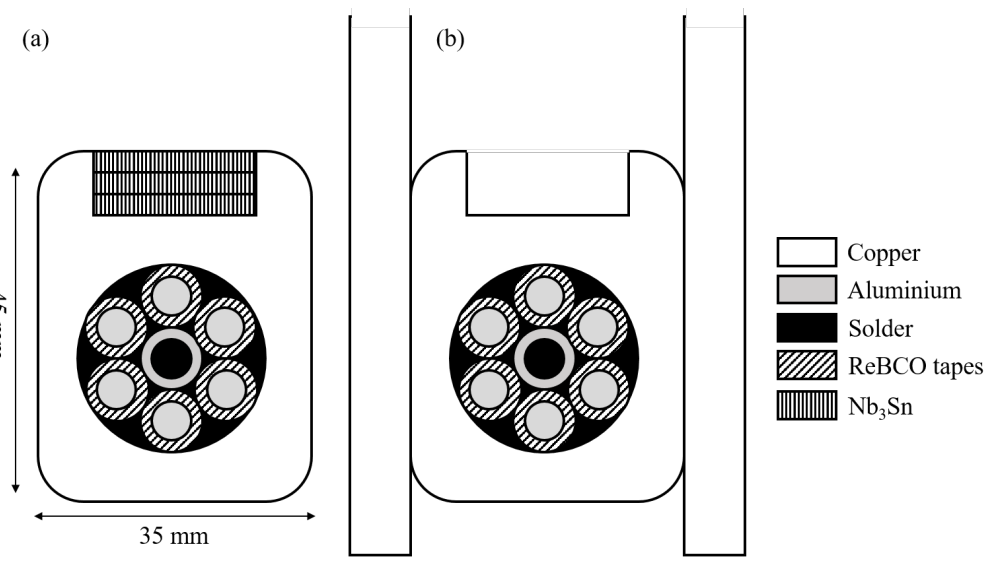

Figure 5.10: Schematic cross-sectional view of the joints of the CICC in both sample holders. Figure (a) shows the splice between the CORC CICC and the stack of $\mathrm{Nb}_{3} \mathrm{Sn}$ conductors during the test at $4.2 \mathrm{~K}$. Figure (b) shows the joint section in the setup for measurement at $77 \mathrm{~K}$. In this case, the joint terminal is clamped between two copper flexible current leads.

\subsubsection{In-field Performance at Liquid Helium Temperature}

The CICC was tested at $4.2 \mathrm{~K}$ in an external magnetic field ranging from 4 to $9 \mathrm{~T}$ and with a transport current up to the $32 \mathrm{kA}$ current limit of the facility. Voltages across each individual strand were measured in the high-field region over a distance of one cable pitch. Current is assumed to be mainly distributed within the joint terminals, given the relatively short length of the sample.

In total 27 measurement runs were performed. The sample was warmed up and cooled down after the sixth measurement run to resolve a sudden short to ground. The electric fields measured as function of overall cable current at $9 \mathrm{~T}$ with a ramp-rate of $400 \mathrm{~A} / \mathrm{s}$ (before warm-up) and at $6 \mathrm{~T}$ and $200 \mathrm{~A} / \mathrm{s}$ (after warm-up) are shown in Figure 5.11. The electric fields over the six strands at $6 \mathrm{~T}$ and $200 \mathrm{~A} / \mathrm{s}$ shows no voltage rise within the noise level of $10 \mu \mathrm{V} / \mathrm{m}$. The measurement run at $9 \mathrm{~T}$ and $400 \mathrm{~A} / \mathrm{s}$ shows more noise, however, no indication of a transition from the superconducting to the normal state is observed. The critical current of the individual strands was never reached during the $4.2 \mathrm{~K}$ test and therefore it can be concluded that the critical current of this conductor beyond the capacity of the measurement facility.

\section{Performance of the Joint Terminals at $4.2 \mathrm{~K}$}

The sample is inserted vertically in the bore of the facility's dipole magnet. Therefore the terminals are referred to as the 'top' and 'bottom' joint terminals. The top terminal makes a 'shaking-hands' type splice to the NbTi current lead, which is connected to the power converter. The bottom terminal makes a 'praying-hands' type splice to a stack of three $\mathrm{Nb}_{3} \mathrm{Sn}$ cables. The voltage measured across the terminal to the CORC cable 

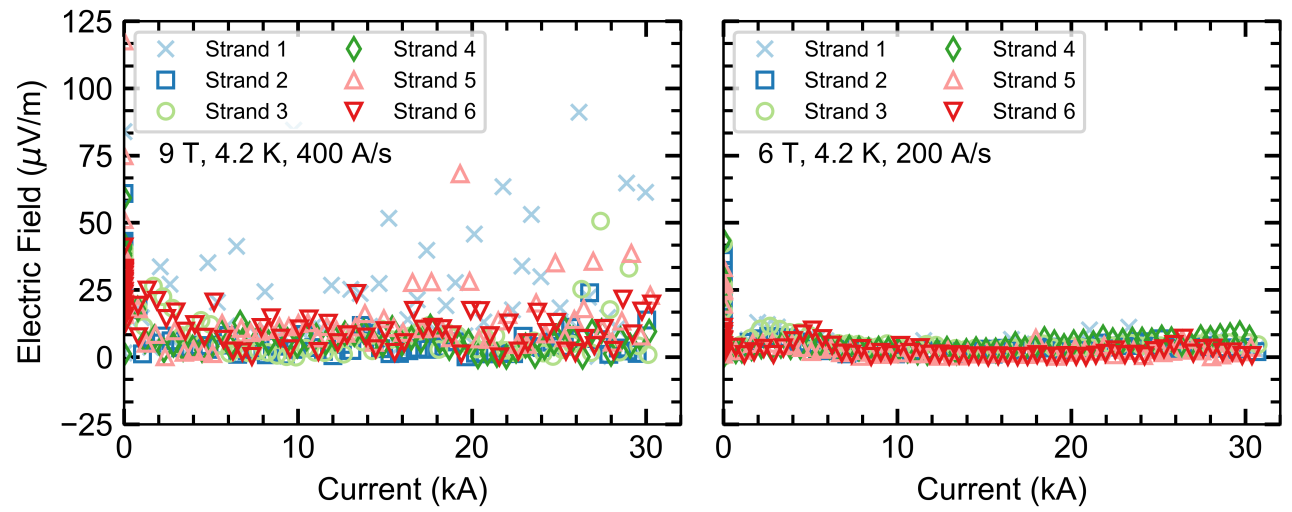

Figure 5.11: Electric fields over the six CORC strands at a temperature of $4.2 \mathrm{~K}$ in an external magnetic field of $9 \mathrm{~T}$ with a ramp-rate of $400 \mathrm{~A} / \mathrm{s}$ and in $6 \mathrm{~T}$ with $200 \mathrm{~A} / \mathrm{s}$.

bundle is presented in Figure 5.12 for magnetic fields of 4 to $9 \mathrm{~T}$ and various ramp-rates. The resistance is calculated using a linear regression between $5 \mathrm{kA}$ and the respective maximum current of that run. The lower limit of $5 \mathrm{kA}$ is chosen since a small change in the slope of the voltage versus current curves is observed at that point. This results in a resistance value about 10\% higher than when voltage data from the entire current range is used. It was impossible to perform a reliable voltage measurement over the bottom joint terminal at magnetic fields lower than $9 \mathrm{~T}$ due to a technical issue with the experimental setup.

Both joint terminals are partly exposed due to their position to the stray field of the dipole magnet. The resistance of both joint terminals rises substantially as function of magnetic field due to the magneto-resistivity of the copper. The resistance of the top terminal rises from $1.9 \mathrm{n} \Omega$ at $5 \mathrm{~T}$ to $2.8 \mathrm{n} \Omega$ at $6 \mathrm{~T}$ and to $3.9 \mathrm{n} \Omega$ at $9 \mathrm{~T}$ with a ramp-rate of $100 \mathrm{~A} / \mathrm{s}$. The measured resistance of $1.9 \mathrm{n} \Omega$ of the top joint terminal at $5 \mathrm{~T}$ and $100 \mathrm{~A} / \mathrm{s}$ is close to the expected joint resistance of $1.5 \mathrm{n} \Omega$, which is another indication that the joint model is able to make accurate predictions. The resistance of the bottom terminal is almost a factor two larger compared to the resistance of the top joint terminal. Such behavior was earlier observed during the characterization measurement of the single CORC strand. In both cases the bottom joint terminal makes a praying-hands splice between sample and current lead. The joint resistance was not measured without external magnetic field. The Lorentz force acting on the CICC and the $\mathrm{Nb}_{3} \mathrm{Sn}$ cable stack in the self-field is then pointing outwards; a direction by which the $\mathrm{Nb}_{3} \mathrm{Sn}$ stack is not supported due to space restrictions.

The resistance of the top joint terminal increased with increasing ramp-rate from $3.9 \mathrm{n} \Omega$ at $4.2 \mathrm{~K}$ and $9 \mathrm{~T}$ with a ramp-rate of $100 \mathrm{~A} / \mathrm{s}$ to $4.5 \mathrm{n} \Omega$ with a ramp-rate of $400 \mathrm{~A} / \mathrm{s}$. The resistance value of bottom joint is higher with a value of $5.8 \mathrm{n} \Omega$ at a ramp-rate of $100 \mathrm{~A} / \mathrm{s}$. However, it decreased to $5.2 \mathrm{n} \Omega$ with an increase of ramp-rate to $400 \mathrm{~A} / \mathrm{s}$. 
Therefore, some inductive effects are present that influence the current distribution within the terminals. The total resistance of the two terminals combined did not change.
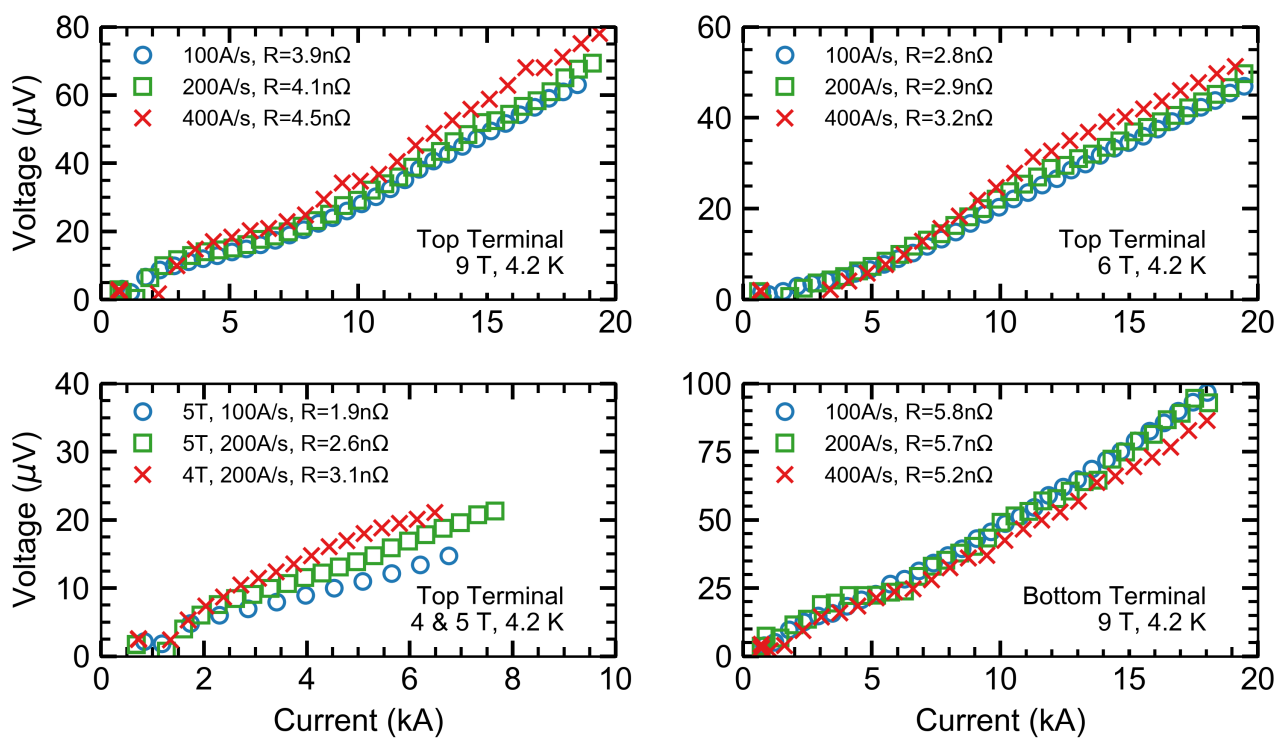

Figure 5.12: Measured voltages over the top and bottom joint terminals as function of current at a temperature of $4.2 \mathrm{~K}$ for various magnetic fields and ramp-rates. The resistance of the top joint terminal rose by increasing magnetic field or ramp-rate. The resistance of the bottom joint terminal decreased by increasing ramp-rate.

\subsubsection{Self-Field Performance at $77 \mathrm{~K}$}

The CICC was tested at $77 \mathrm{~K}$ in self-field up to a current of $15 \mathrm{kA}$ with ramp-rates between 100 and $1500 \mathrm{~A} / \mathrm{s}$. The cable has a critical current of 12.3 to $13.0 \mathrm{kA}$ at $77 \mathrm{~K}$, depending on the ramp-rate, in a self-field of about $0.4 \mathrm{~T}$. These values are conform to the predicted critical current of $13 \mathrm{kA}$. The $\mathrm{n}$-value of the CICC ranges from 12.3 to 15.3. A higher critical current, but lower n-value, is found at higher ramp-rates. An overview of the conductor's critical current and n-value as function of ramp-rate is presented in Figure 5.13.

The superconducting to normal transition of each CORC strand in the bundle is shown in Figure 5.13. The cable reaches its $I_{c}$ at $12.3 \pm 0.2 \mathrm{kA}$ at a $I_{c}$ criterion of $1 \mu \mathrm{m} / \mathrm{cm}$, which is a convincing demonstration of overall uniform current distribution between strands.

Each test was performed up to a current of $15 \mathrm{kA}$. No thermal run-away on the CICC was observed, nor excessive boiling of the nitrogen up to at least $15 \mathrm{kA}$, thus $2 \mathrm{kA}$ over the conductor's critical current. This demonstrates that the conductor is electrically and 
thermally very stable, even when operated well over its critical current, when cooling is sufficient.
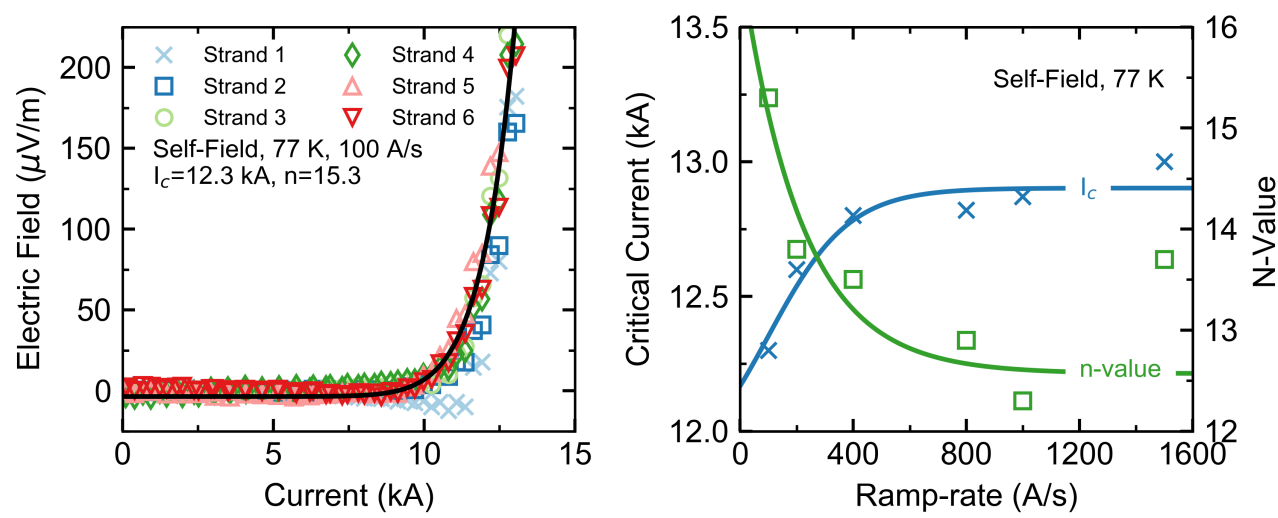

Figure 5.13: Test results of the first CORC CICC at $77 \mathrm{~K}$ in self-field. The graph on the left shows the electric field over each CORC strand in the center of the CICC as function of current. The transition from the superconducting to the normal state starts at a similar current for each CORC strand. A critical current of $12.3 \pm 0.2 \mathrm{kA}$ is measured by fitting a power-law through the average electric fields of the strands with the $I_{c}$ criterion of $1 \mu \mathrm{m} / \mathrm{cm}$. The resulting $I_{c}$ and $n$-values of the CORC CICC as function of ramp-rate are presented in the graph on the right.

\subsubsection{Performance of the Joint Terminals at $77 \mathrm{~K}$}

The 'top' terminal has a constant resistance of $6.8 \pm 0.1 \mathrm{n} \Omega$ and the 'bottom' terminal has a resistance of $9.9 \pm 0.2 \mathrm{n} \Omega$ up to a current of about $10 \mathrm{kA}$, independent of the current ramp-rate. Both terminals have a resistance that is about $4 \mathrm{n} \Omega$ higher than the values found at $4.2 \mathrm{~K}$, which corresponds to the expected resistance rise of the copper and solder material in the terminal. The onset of transition of the strands within the joint terminals starts at about $10 \mathrm{kA}$, as shown in Figure 5.14, which is consistent with the superconducting-to-normal transition of the main conductor.

In accordance with the measurements at $4.2 \mathrm{~K}$, the resistance of the bottom joint terminal is higher than that of the top terminal. After the measurements at $4.2 \mathrm{~K}$ it was expected that the difference was due to the difference in type of splice to the sample holder's current leads. However, that was not the case at $77 \mathrm{~K}$, since both terminals had the same clamped type connections to the flexible copper leads.

\subsubsection{Discussion}

The CICC and its joint terminals perform close to expectations at both 4.2 and $77 \mathrm{~K}$. The measured critical current of the conductor at $77 \mathrm{~K}$ is used as benchmark $\mathrm{I}_{c}$ to extrapolate 

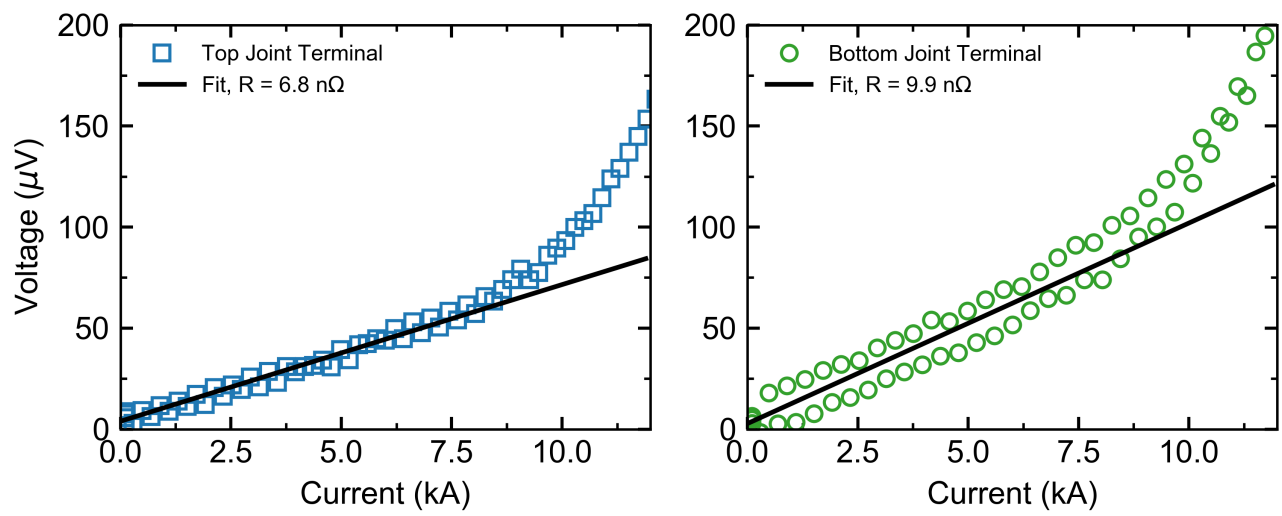

Figure 5.14: Measured voltages over the top and bottom joint terminals as function of current for a ramp-rate of $400 \mathrm{~A} / \mathrm{s}$. A higher inductive voltage is measured over the bottom joint terminal compared to the top joint terminal. A transition from the superconducting to the normal state is observed in both joint terminals starting around $10 \mathrm{kA}$, consistent with the transition of the main conductor.

the conductor's critical current up to a magnetic field of $20 \mathrm{~T}$ for various temperatures, as shown in Figure 5.15. The critical current at $4.2 \mathrm{~K}$ and $20 \mathrm{~T}$ is expected to be approximately $31 \mathrm{kA}$. At a temperature of $40 \mathrm{~K}$ and a magnetic field of $5 \mathrm{~T}$ (a characteristic operating magnetic field of a detector magnet) it can carry $26 \mathrm{kA}$. Therefore, the CORC CICC is indeed a promising conductor for future large magnets operated in the $20-50 \mathrm{~K}$ range.

The resistance of the 'top' joint terminal matches the predicted resistance at both 4.2 and $77 \mathrm{~K}$, which is a clear indication that the joint-model used to estimate the joint resistance is able to correctly predict the resistance of this type of CORC joint terminals. The 'bottom' joint terminal has a slightly larger resistance at both temperatures. A possible explanation for the higher resistance values is that there are still some voids present in the solder between the CORC strands and terminal, which directly leads to a higher resistance.

It is observed that the resistance of the terminals changes slightly as function of current ramp-rate at $4.2 \mathrm{~K}$. The mutual inductance between strands forces a more equal current distribution in the cable and the joints. Small variation in contact resistance between strands become less pronounced at higher ramp-rate and current is distributed more uniformly. Still, no transition of individual strands is observed at $4.5 \mathrm{~K}$ and only a minor difference in critical current between strands is seen at $77 \mathrm{~K}$.

Several thermal cycles occurred between the various tests. No degradation of the conductor due to these cycles, cabling, closing of the jacket, handling and the tests in high magnetic field has been observed. 


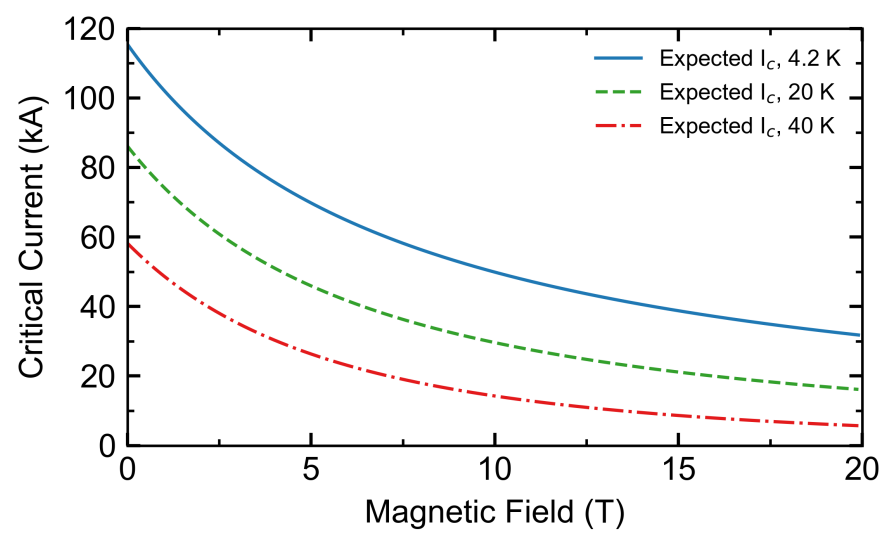

Figure 5.15: Calculated critical current of the CICC as function of magnetic field and temperature, using the experimentally determined $I_{c}(77 K$, self-field) as reference.

\subsubsection{Conclusion}

World's first ReBCO CORC six-around-one Cable-In-Conduit Conductor was tested at $4.2 \mathrm{~K}$ in a magnetic field of $9 \mathrm{~T}$ and at $77 \mathrm{~K}$ in self-field. At $4.2 \mathrm{~K}$ and $9 \mathrm{~T}$ the conductor remains superconducting up to $32 \mathrm{kA}$, the compliance current of the test facility. The resistance of one joint terminal at $4.2 \mathrm{~K}$ for various values of magnetic fields and ramp-rates is 2 to $4 \mathrm{n} \Omega$. The other terminal has a slightly higher resistance of 5 to $6 \mathrm{n} \Omega$. At $77 \mathrm{~K}$, the critical current of the conductor is 12.3 to $13.0 \mathrm{kA}$ with an n-value of 15.3 to 12.3 , respectively, depending on the ramp-rate. The joint terminals have a resistance of 6.8 and $9.9 \mathrm{n} \Omega$ at $77 \mathrm{~K}$. The critical current and joint terminal resistance values at both 4.2 and $77 \mathrm{~K}$ are conform to expectations and no degradation due to thermal cycling, cabling, jacketing, joint fabrication nor to the test in high magnetic field itself was observed.

This first six-around-one CORC CICC and its joint terminals may thus be said to have demonstrated a promising performance and can be seen as a successful first step in the development of high-current CORC Cable-In-Conduit technology. 


\subsection{Two New CORC CICC Samples for Magnets aiming at Fusion Experiments and Particle Detectors}

The development of CORC Cable-In-Conduit Conductors is continued with the preparation of two new and significantly improved CICCs, featuring a higher design current compared to the first demonstrator CICC and forced-flow gas cooling. The first new conductor is designed especially for use in demanding high magnetic field-, high heat load and high mechanical stress environments such as found, for example, in tokamak-type fusion magnets. The second conductor is designed for high thermal- and electrical stability and conduction type cooling, as required in detector magnets and superconducting bus bars. Schematic cross-sections of both conductors are shown in Figure 5.16. The two conductors were assembled at CERN in collaboration with Advanced Conductor Technologies. Both conductors were tested in the SULTAN cable test facility at PSI, Villigen, Switzerland in the second half of 2017.

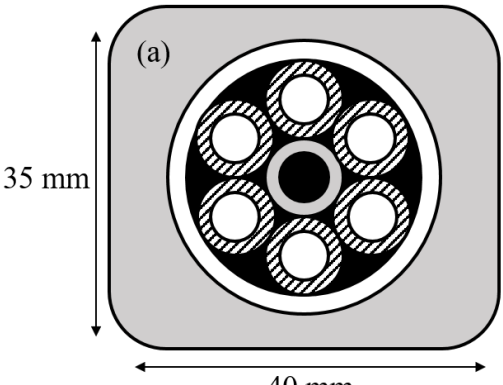

$40 \mathrm{~mm}$

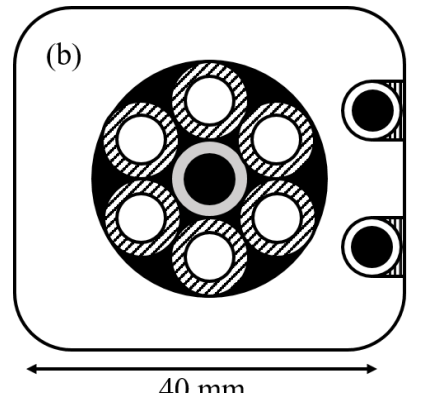

$40 \mathrm{~mm}$

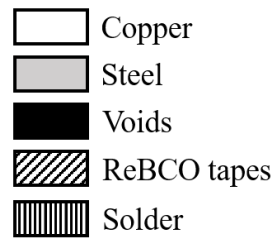

Figure 5.16: CORC Cable-In-Conduit Conductor cross-sections with (a) stainless steel jacket for fusion magnets and (b) copper jacket for detector magnets and bus bars, the latter including two additional cooling channels for conduction cooling.

\subsubsection{Layout of the Two CORC Six-Around-One CICCs}

Both CICCs use the six-around-one cabling layout in which six CORC strands are helically twisted around, in this case, a stainless steel tube. Each strand contains 42 SCS4050 ReBCO tapes from SuperPower Inc., for a total of 252 tapes. The tapes have only $5 \mu \mathrm{m}$ of copper plating on each side, which greatly increases their overall current density compared to the tapes in the previous CORC CICC, which had $20 \mu \mathrm{m}$ of copper plating on each side.

The CORC strands in the two CICCs are slightly different in design. The core of the strands for the fusion-type CICC is a solid copper former $5 \mathrm{~mm}$ in diameter, while the strands for the detector-type CICC have a solid copper former of $4 \mathrm{~mm}$ in diameter that is extended to a diameter of $5 \mathrm{~mm}$ using 5 layers of copper tape. The thinner solid-copper core makes the second type of strand more flexible and thus easier to bundle into the 


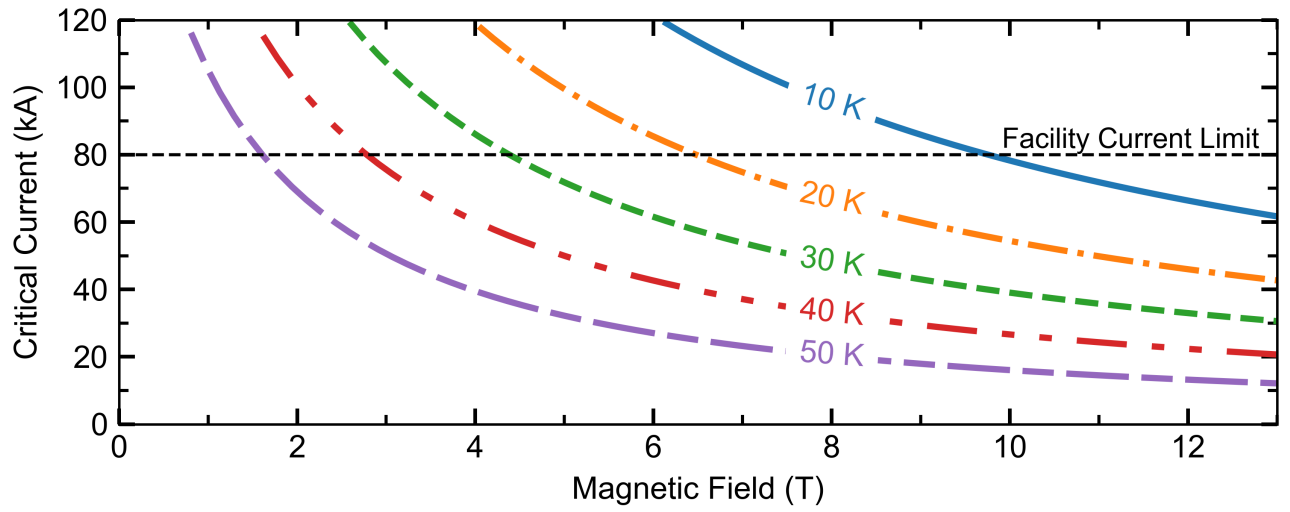

Figure 5.17: Projected cable critical current versus magnetic field, $I_{c}(B)$, for various operating temperatures of the CICCs tested in the SULTAN facility. The measurement station has a current supply limit of $80 \mathrm{kA}$ and therefore it is not possible to measure $I_{c}(B)$ at low applied magnetic field and low temperatures.

six-around-one cable. However, these strands were more springy and harder to keep in place after bundling than the strands with the thicker, more rigid core.

The ReBCO tapes are wound in 14 layers around the stand's core, followed by two additional layers of copper tapes and a layer of polyester insulation. The outer diameter of each strand is $7.6 \mathrm{~mm}$. The twisting of the six-around-one cable was performed manually. The twist starts at a distance of around $150 \mathrm{~mm}$ from the joint section. The cable pitch length is $400 \mathrm{~mm}$, allowing for a full pitch length to be situated within the high-field region of the SULTAN facility. In total, there are 4.5 cable pitches between the two joint terminals.

The polyester insulation around the CORC strands protects the tapes during twisting. This insulation is removed after twisting to allow direct contact with cold helium gas and to allow electrical contact between strands. The diameter of each six-around-one cable bundle is extended to $24 \mathrm{~mm}$ with several layers of $50 \mu \mathrm{m}$ thick and $40 \mathrm{~mm}$ wide copper foil. The copper foil provides mechanical protection of the bundle and ensures a tight fit of the cable within the jacket.

Both six-around-one cable bundles are designed to carry $80 \mathrm{kA}$ at $12 \mathrm{~T}$ and $4.2 \mathrm{~K}$, which corresponds to an overall current density in the cable bundle, with a diameter of $24 \mathrm{~mm}$, of $177 \mathrm{~A} / \mathrm{mm}^{2}$. The critical current is calculated using the scaling methods described in Section 4.2. The calculated engineering current density of the entire conductor, including the jacket sized, is $57 \mathrm{~A} / \mathrm{mm}^{2}$. The expected critical current as function of magnetic field and temperature of the two conductors is presented in Figure 5.17.

A detailed overview of the conductor properties is presented in Table 5.2. For comparison, the table also includes the corresponding details on the first CORC CICC based conductor 


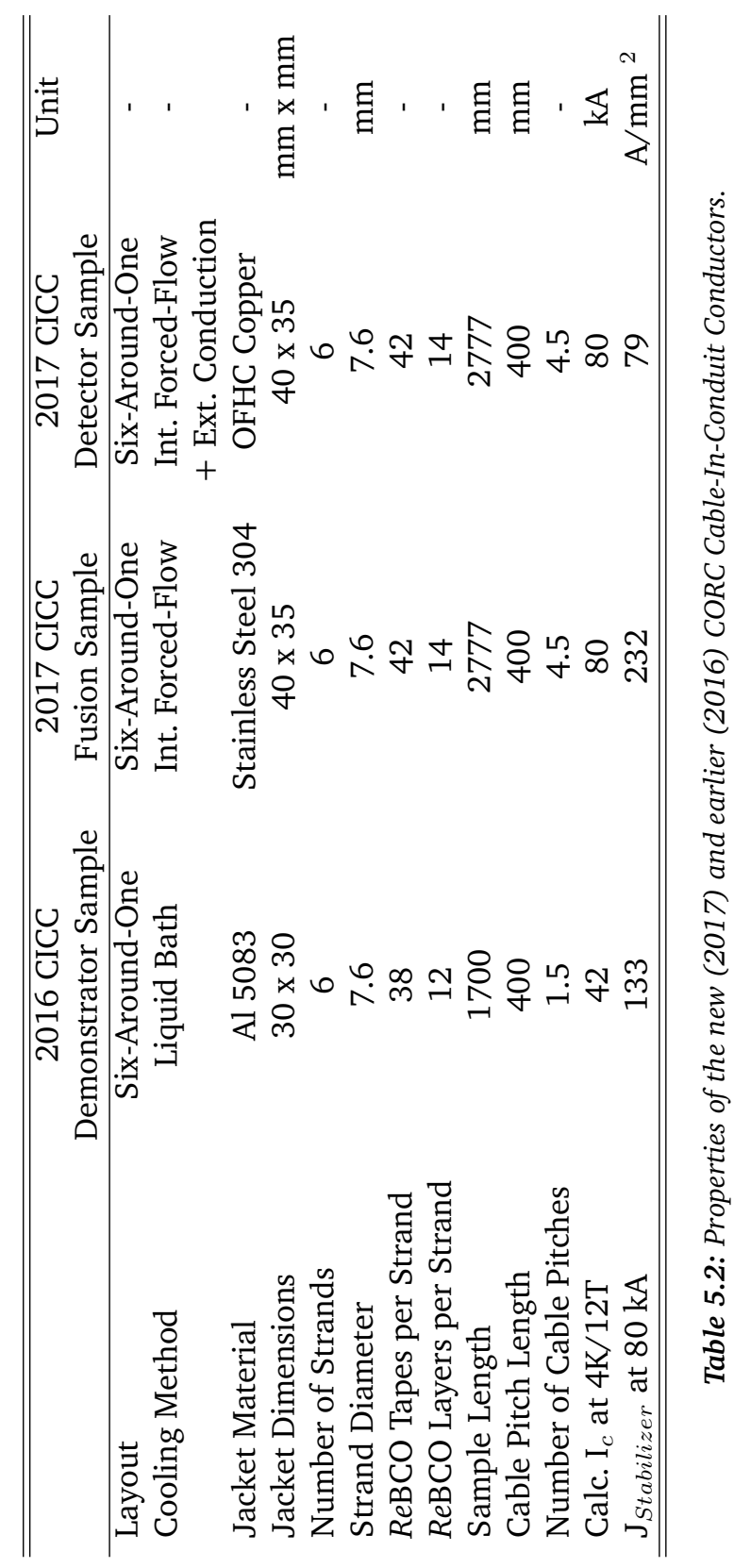


that was tested in 2016 at CERN, illustrating the evolution in size and performance of the CORC CICCs.

\subsubsection{Jacketing of the CORC Cable-In-Conduit Conductors}

The fusion-type CORC CICC has a stainless steel 314 jacket and internal forced-flow cooling. High-strength materials, such as stainless steel, are required in modern tokamak magnets to take up and transfer the considerable accumulated Lorentz forces that act on the superconducting cables during operation. The high heat load from fusion heating on the conductor requires internal flow cooling, since conduction cooling through the steel jacket is negligible. The bore of the stainless steel jacket is $28 \mathrm{~mm}$ in diameter. The six-around-one CORC cable is enclosed by an OFHC copper tube with a wall thickness of $2 \mathrm{~mm}$ for a total cable outer diameter of $28 \mathrm{~mm}$. The copper tube is soldered into the copper joint terminals to provide a parallel path for the current if necessary. The CICC and the six-around-one cable at two different stages of assembly are shown in Figure 5.18.
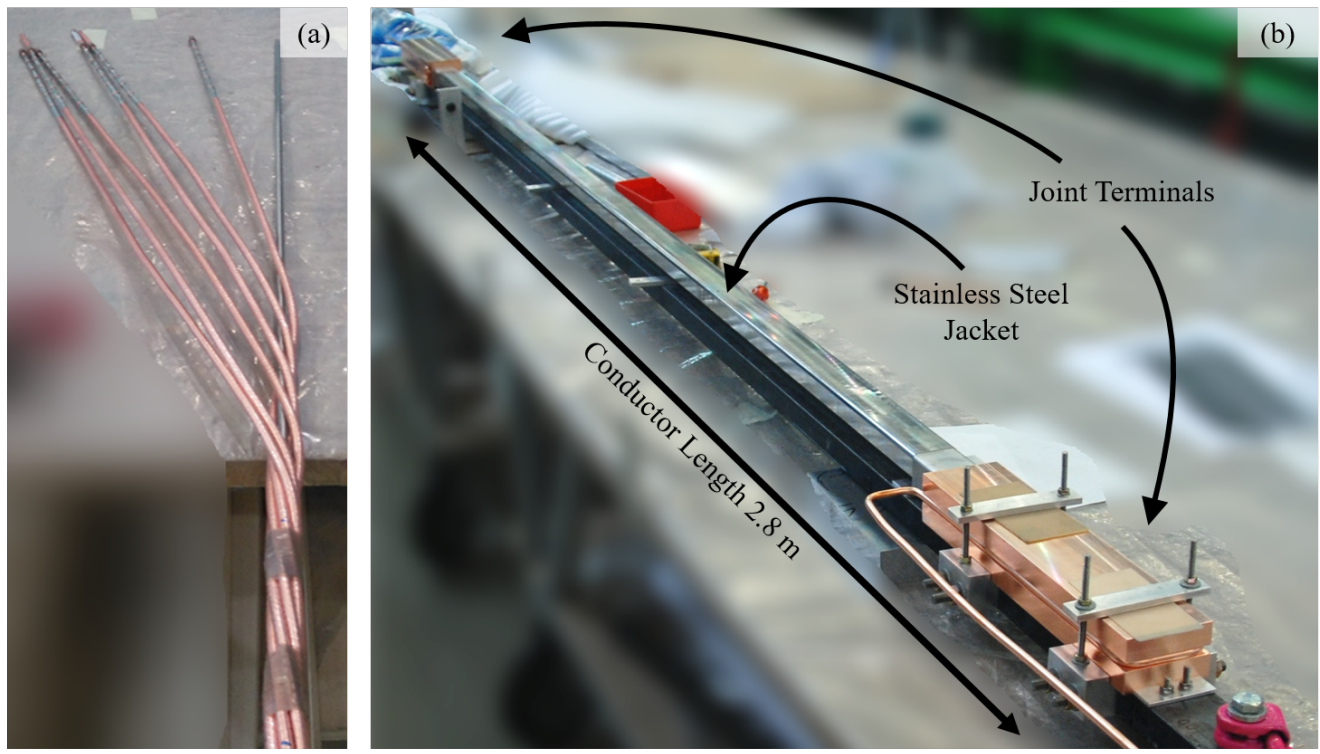

Figure 5.18: (a) Winding of the six-around-one CORC cable performed manually around a stainless steel tube. (b) The CORC CICC with the two joint terminals and the stainless steel jacket during assembly. The conductor is mounted on a stainless steel bar during assembly to ensure that both joint terminals are mounted parallel.

Lorentz forces acting on the conductors of detector magnets and their bus lines are much lower compared to the forces in fusion type magnets. Therefore, a material with lower strength but higher thermal- and electrical conductivity can be used as jacket for the six-around-one CORC detector-type cable. In practice, aluminium is commonly used in conductors for detector magnets due to its high radiation transparency. OFHC copper as 
stabilizer material is also not uncommon in conductors for detector magnets, but better suited for their bus bars. The high thermal conductivity of these materials opens the possibility of a conduction-cooled conductor and greatly improves its thermal and electrical stability. Conduction-cooling is preferred for its simplicity and practical implementation. The detector-type CORC CICC sample has an OFHC copper jacket with a bore of $24 \mathrm{~mm}$ in diameter and with two helium lines integrated in the jacket for conduction cooling, as well as the option of internal forced-flow cooling via an additional helium line entering the jacket near the joint terminals. The cooling scheme for the CORC CICC samples is presented in Figure 5.19.

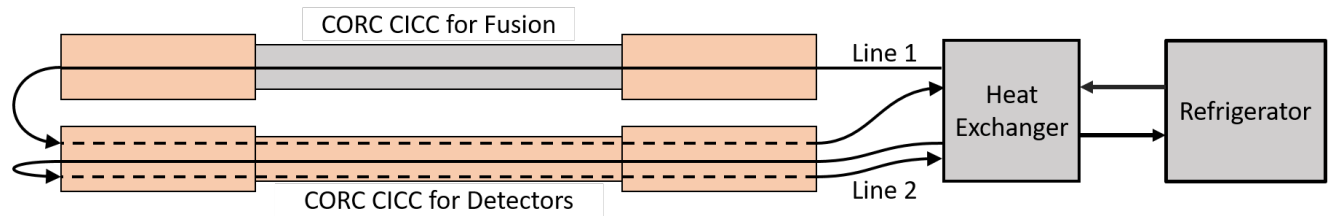

Figure 5.19: Schematic of the two cooling channels including the heat-exchanger. The temperature of the helium gas in each channel is individually regulated by heaters and the heat exchanger. The mass-flow in each channel can be regulated. Line 1 applies internal forced-flow cooling (solid line) to the CORC CICC with the stainless steel jacket and returns as a conduction-cooling line (dashed line) for the CORC CICC with copper jacket. The optional line 2 supplies additional forced-flow cooling to the CORC CICC with copper jacket if necessary and the gas returns via a tube embedded in the copper jacket.

Copper was chosen over aluminium alloy in the detector-type CICC sample because it more closely matches the thermal expansion of the stainless steel jacket of the fusiontype CICC, since both samples are measured in series, mounted alongside each other in the same sample holder. The copper material of the jacket is cold-worked, high tensile strength OFHC copper, since stress levels up to $200 \mathrm{MPa}$ are expected in the jacket during the characterization test, exceeding the yield strength of fully annealed OFHC copper. In practice, the copper jacket can be fully annealed material if this conductor were to be used in a low-stress environment such as in a detector magnet or its bus lines. The peak stress in the stainless steel jacket is well below its yield strength during the characterization test. Pictures of short trial samples of both conduits are presented in Figure 5.20.

Each jacket is lengthwise divided in two halves. The halves of the jackets are assembled and welded using electron-beam welding for both the stainless steel- and the copper jacket. A bimetallic connection between copper joint terminals and stainless steel jacket is created by brazing a $50 \mathrm{~mm}$ long stainless steel adaptor piece to the joint terminals. The six-around-one CORC cable was inserted via the adaptor to the terminal. Access to the terminal for soldering was possible via the small gaps between CORC strands. The CORC CICC with the copper jacket has similar adaptor pieces to provide symmetry between the two conductors. The adaptor pieces also serve as in- and outlet points for the internal gas flow cooling. The adaptors are shown in Figure 5.21. 
(a) Stainless Steel Jacket

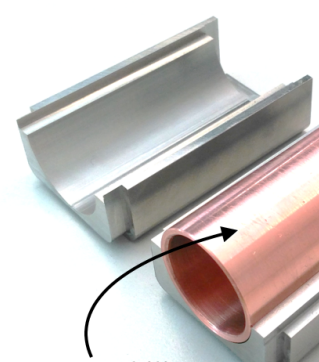

Copper Stabilizer (b)

Copper Jacket

Figure 5.20: Photos of two trial jacket pieces used for testing the assembly and welding procedures.

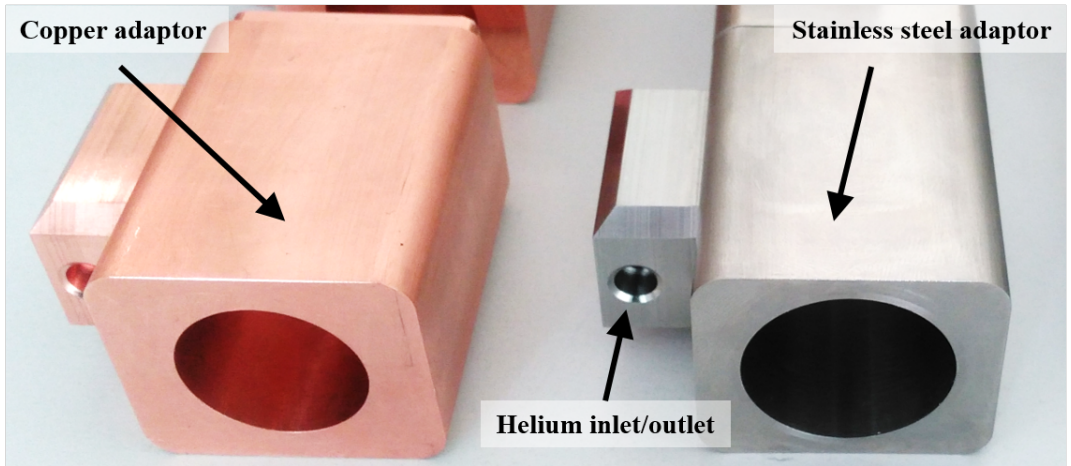

Figure 5.21: The picture shows the copper and stainless steel adaptor pieces before assembly onto the conductor. The adaptor pieces are later brazed to the copper joint terminals and form the link between terminal and jacket. The jackets are welded to the adaptor pieces using electron beam welding. The adaptors also serve as inlet and outlet points for the internal forced-flow cooling.

\subsubsection{Joint Terminal Layout and Optimization}

The joint terminals are designed to furnish a low-resistance connection that distributes current equally between CORC stands. The calculated joint terminal resistance is about $2 \mathrm{n} \Omega$ per terminal at $4.5 \mathrm{~K}$, adding up to a total loop resistance of $8 \mathrm{n} \Omega$. At $50 \mathrm{~K}$, the expected resistance is approximately $6 \mathrm{n} \Omega$ per terminal, giving a total loop resistance of $24 \mathrm{n} \Omega$. The copper joint terminals are $305 \mathrm{~mm}$ long, in which the CORC strands are inserted over a length of $285 \mathrm{~mm}$.

The connection from CORC strands to terminals is made in a similar way as in the demonstrator (2016) CORC CICC sample. The entire terminal is heated to about $196 \pm 2^{\circ} \mathrm{C}$ and filled with eutectic Sn63Pb37 solder. The ends of the CORC strands are staged to a staircase-like geometry and solder-coated. Twisting of the strands within the terminal was impossible due to the stiffness of the solder coating on the strand ends, therefore they remain straight in the terminals. The cooling tubes are soldered in a groove on the surface 
of the joint terminals in the last step of the CICC assembly. Soldering of the cooling tubes is performed with a relatively low-temperature solder (Sn44In42Cd14), such that the solder inside the terminals is not affected.

\subsubsection{Test Setup of the Two CORC CICCs}

The two CORC Cable-In-Conduit Conductors were tested in the SULTAN test facility at PSI, Villigen, Switzerland. This test facility features a 11 T DC dipole magnet, a sample temperature range between 4.5 and $60 \mathrm{~K}$ and a dipole magnet that is able to generate an AC transverse field of $\pm 0.4 \mathrm{~T}$ with a maximum frequency of $10 \mathrm{~Hz}$. The two CORC CICCs were tested in a hairpin-type sample holder, electrically connected in series. One of the two CICCs carried the current through the facility's high-field region and the other CICC carried it back. On one side of the sample holder, both CICCs were connected to the so-called HTS adaptor of the SULTAN setup, which injects current in one sample and extracts it from the other. The two conductors make a 'praying-hands' splice on the other side of the sample holder. A schematic view of the sample holder and the position of the instrumentation is shown in Figure 5.22.

As discussed above, one CICC was designed for application in fusion-type magnets with forced-flow cooling and the other was designed for detector-type magnets with conduction cooling. However, it was uncertain if conduction cooling would be sufficient, therefore the CORC CICC with the copper jacket also comprised a channel for optional forced-flow cooling, as shown in Figure 5.19. Both CORC CICCs can be cooled with only cooling channel 1 in operation. Channel one provides forced-flow cooling to the CORC CICC for fusion and conduction cooling to the CORC CICC for detector magnets. Channel 2 can provide additional forced-flow cooling to the CORC CICC for detector magnets in the case conduction cooling is not sufficient.

The Lorentz forces during the test are pointing outwards due to the orientation of the self-field and the external magnetic field. Therefore, an aluminium clamping system keeps both CORC CICCs together. Both conductors are wrapped in polyamide tape and have a layer of G10 insulation in between them. The peak self-field is $1.05 \mathrm{~T}$ per $50 \mathrm{kA}$, as illustrated by Figure 5.23.

\subsubsection{Results of the Two CORC Cable-In-Conduit Conductors}

Both conductors were tested during two test campaigns with a thermal cycle in-between. Both were able to carry $80 \mathrm{kA}$ in self-field at a temperature of $5 \mathrm{~K}$ during the first control measurements in both cool-down campaigns, as shown in Figure 5.24. The measurement at $5 \mathrm{~K}$ without external field shows a systematic voltage rise as function of current over the high-field section of both CICC samples, visible both in the level of the flat DC 'steps' corresponding to the current plateaus and in the slew of the voltage during actual ramping of the current. This voltage may indirectly originate from the joint terminals of the samples. An imperfect contact between the jacket and the cable bundle can result in parasitic voltages over the jacket. The jacket is then not at the same potential as with 


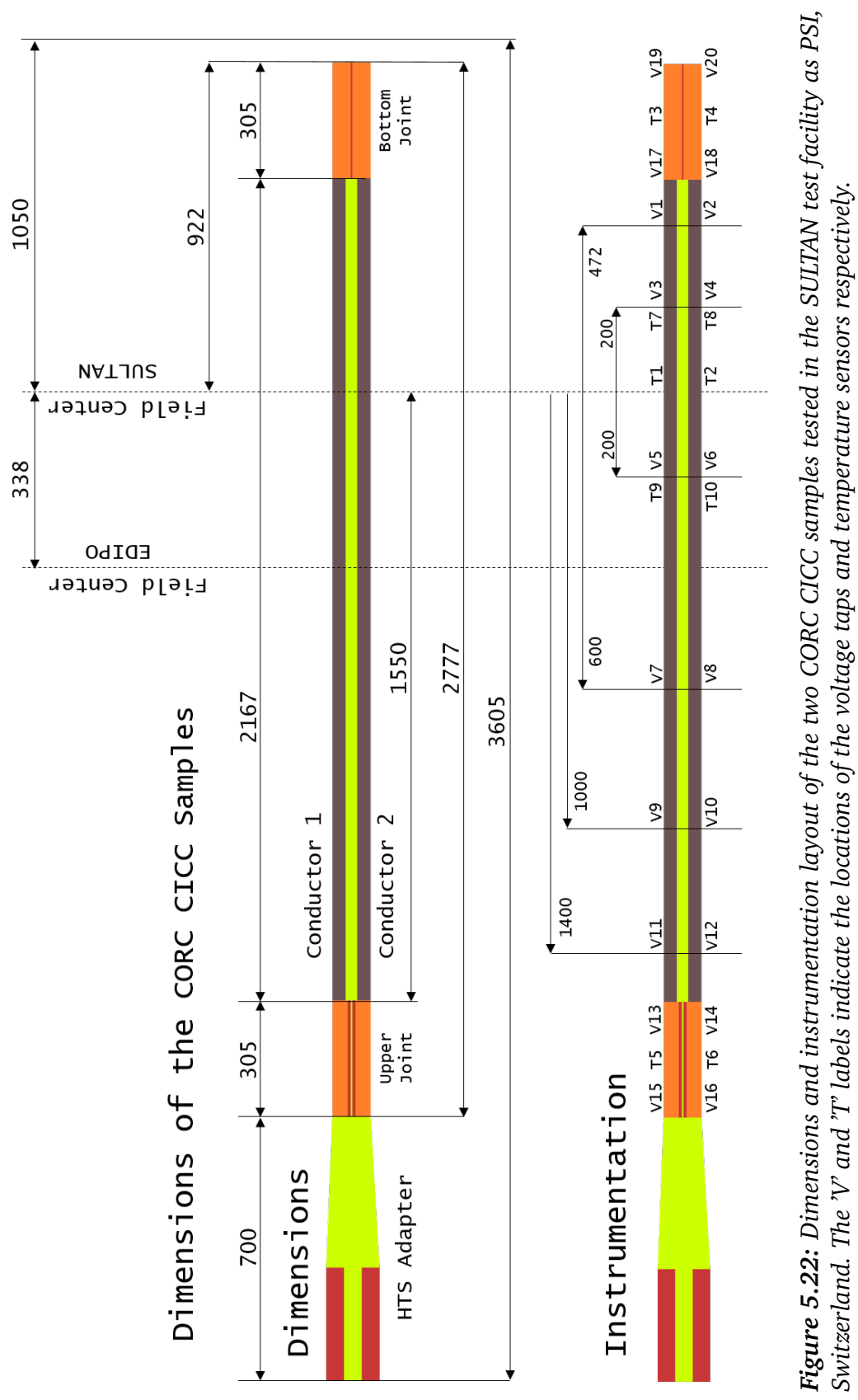




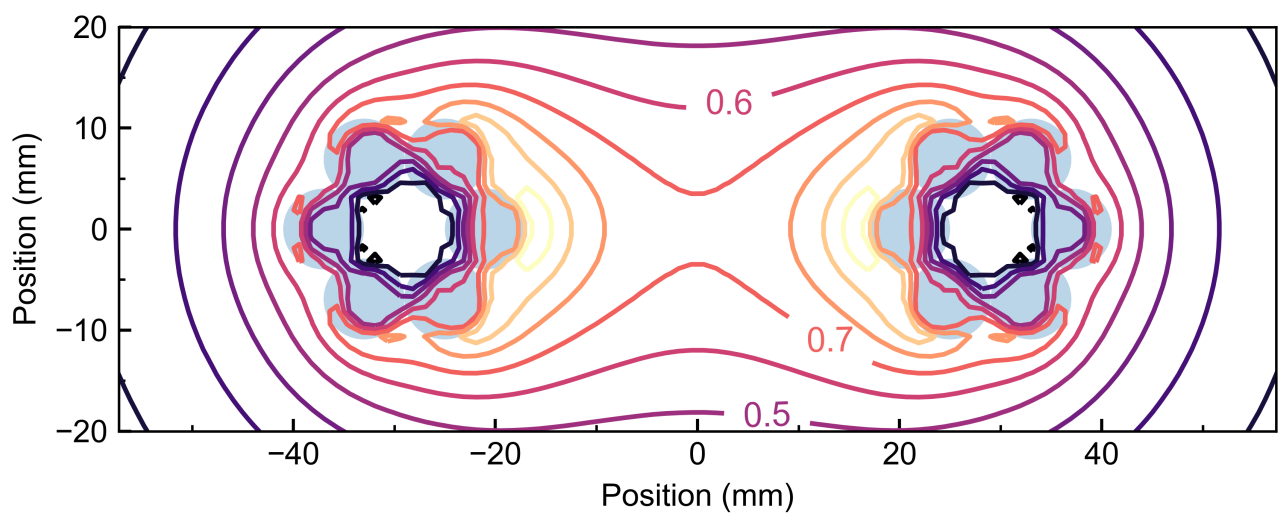

Figure 5.23: Self-field component of the two CORC CICCs at a current of $50 \mathrm{kA}$. The peak self-field on the CORC strands closest to the center of the sample holder is $1.05 \mathrm{~T}$ per $50 \mathrm{kA}$.

the cable bundle, but acts more as a shunt over the conductor length. In such case, if a voltage near the joint terminals builds up due to e.g. current redistribution near the joint terminals, this voltage drop may spread evenly along the length of the jacket.

Another possible explanation for the voltage that was observed to develop across the main conductor section is that some current redistribution occurred over the entire conductor length throughout the entire current range. However, this scenario is very unlikely as it implies that both cable bundles of each 252 ReBCO tapes would have a resistive component already starting at current levels as low as $0 \mathrm{~A}$. The measured resistances were retracted from the voltage signal over the main conductor and added to the voltage over the joint terminal to provide a correct representation of the splice resistance and performance of the conductor.

A similar situation was observed for the non-linear part of the current-voltage relation corresponding to the superconducting to normal state transition of the cable bundle in the high-field region. This voltage is also spread along the length of jacket in both samples. Therefore the non-linear voltages corresponding to this transition measured across, but also next to, the high-field region are summed and assumed to primarily originate in the high-field region.

The temperature during the first control measurement rose to 10 and $14 \mathrm{~K}$ for the SSjacketed and Cu-jacketed CICC, respectively. The mean heat source was the splice connecting the bottom joint terminals of the two conductors, which has a resistance of 17 $\mathrm{n} \Omega$. The helium, heated by the bottom joint, flows towards the main conductor section of the Cu-jacketed CICC thereby increasing its temperature significantly. In this run, this temperature was somewhat mitigated by the helium flow in the optional second cooling path (Figure 5.19).

During the second test campaign, current is ramped up to $80 \mathrm{kA}$ in steps of $10 \mathrm{kA}$. The maximum temperature of both conductors during this run is higher than in the first run, 

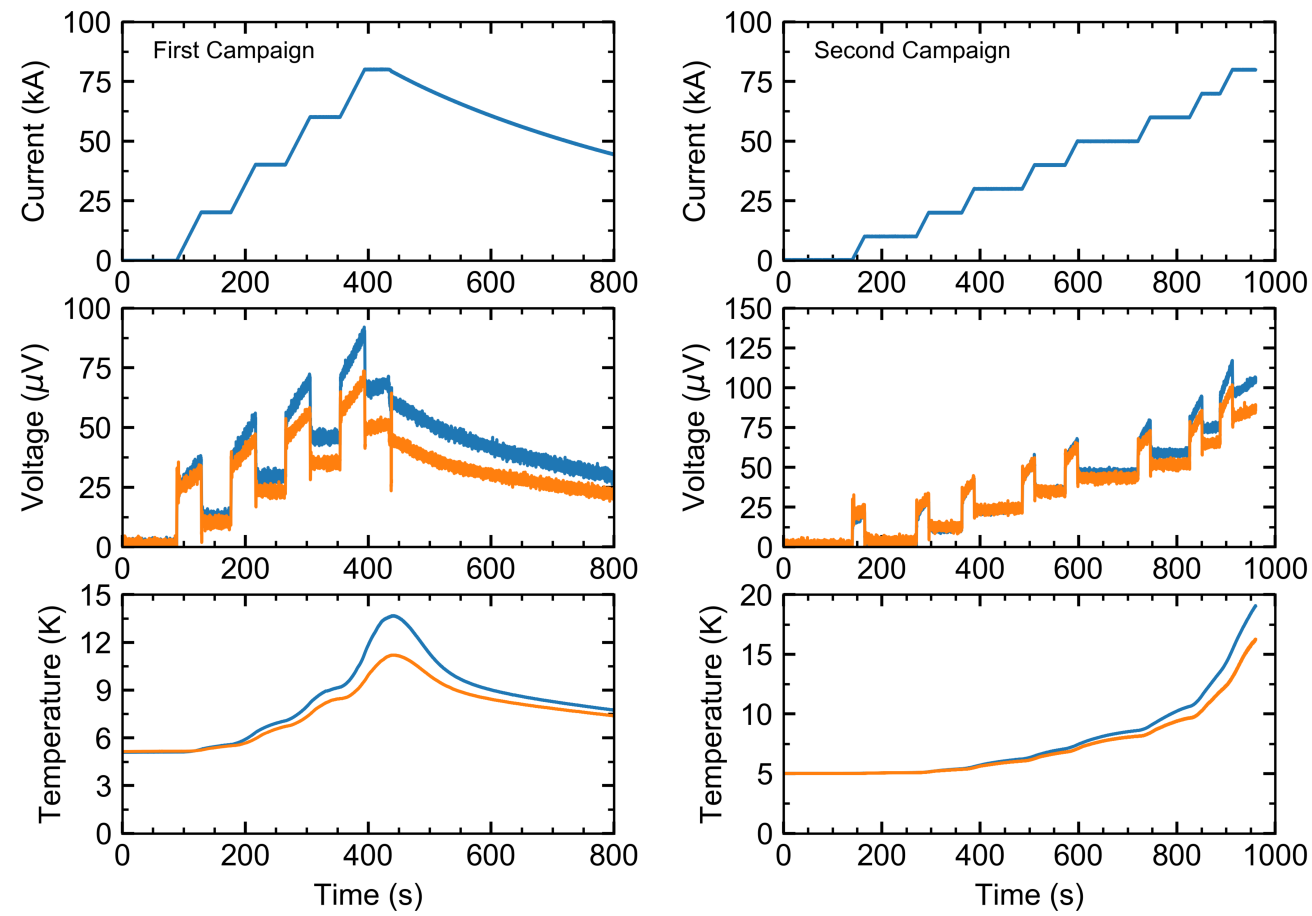

Figure 5.24: Current, voltage over the high-field region (using voltage tap pairs V3-V5 and V4-V6) and temperature (measured with sensors 'T9' and 'T10' indicated in Figure 5.23) of the two conductor samples during the first test of the first (left) and second (right) campaigns. The blue curves correspond to the 'detector-type' copper-jacketed cable and the orange ones to the 'fusion-type' sample with the steel jacket.

at a temperature of 16 and $19 \mathrm{~K}$ for the fusion and detector CICC, because the current is kept stable at 4 additional plateaus, thereby increasing the measurement time and thus the amount of released heat. The resistivity of the copper in the joints increases as function of temperature. This is observed in the voltage trace measured over the jacket as a non-zero slope of the voltage vs. time curve during the plateaus at 70 and $80 \mathrm{kA}$, correlated with the observed rise in temperature.

\section{In-Field Performance of both CORC CIC-Conductors}

Both conductors were extensively tested in the temperature range from 5 to $60 \mathrm{~K}$ in external magnetic fields up to $10.9 \mathrm{~T}$. The Cu-jacketed CICC had a lower critical current than expected over the entire temperature- and magnetic field range. E.g. at $5 \mathrm{~K}$ in an external magnetic field of $10.9 \mathrm{~T}$, the critical current of this conductor is measured to be $37 \pm 4 \mathrm{kA}$, i.e. only 30 to $40 \%$ of its expected value. The SS-jacketed CICC, on the other hand, did not show a reduced performance, but instead has a critical current is close to the predicted value. Unfortunately, in the low-temperature range of 5 to $30 \mathrm{~K}$ 
it was only possible to characterize the Cu-jacketed CICC, since this conductor would quench at current levels far below the transition current of the SS-jacketed CICC, shown in Figure 5.25.
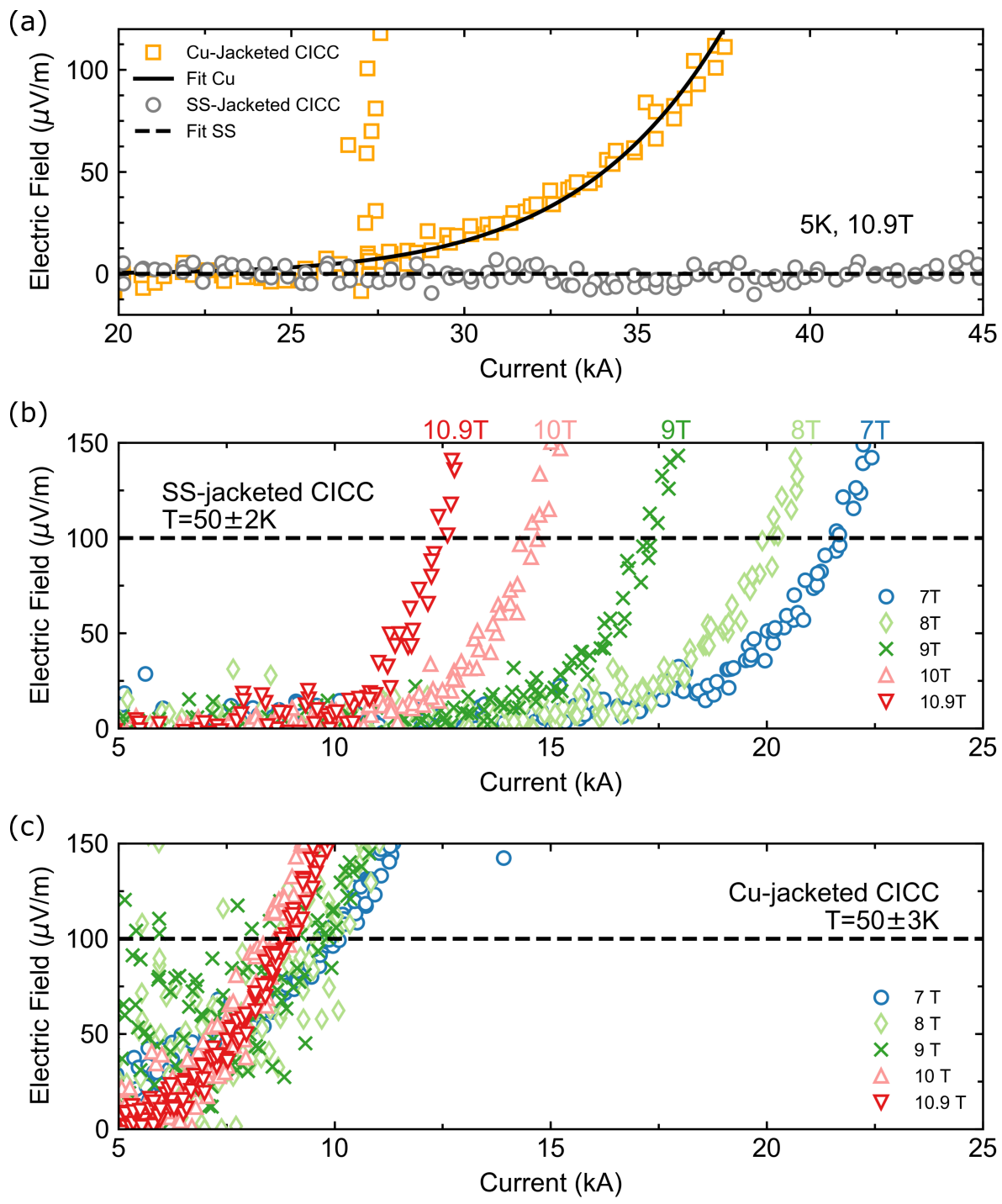

Figure 5.25: Electric fields over measured in the high-field region of both conductors. Figure (a) shows the electric field as function of current at $5 \mathrm{~K}$ and an external magnetic field of $10.9 \mathrm{~T}$. The $I_{c}$ of the $\mathrm{Cu}$-jacketed CICC is approximately $37 \mathrm{kA}$ at $5 \mathrm{~K}$ and $10.9 \mathrm{~T}$ external field with the $\mathrm{I}_{c}$ criterion of $1 \mu \mathrm{m} / \mathrm{cm}$. The critical current of SS-jacketed CICC could not be reached as the Cu-jacketed sample showed thermal runaway at $50 \mathrm{kA}$. Figures (b) and (c) show the electric field along the SS-jacketed CICC and the Cu-jacketed CICC, respectively, at a temperature of $50 \mathrm{~K}$ in various background fields. 
Above $40 \mathrm{~K}$, the heat capacity of both conductors is sufficiently large to allow critical current measurements on both conductors before thermal runaway of the Cu-jacketed CICC occurs. The measured critical currents of both conductors for both measurement campaigns at a temperature of 40, 45 and $50 \mathrm{~K}$ are presented in Figure 5.26. The n-values of both conductors across the measured temperature range are given in Figure 5.27. The critical current of the SS-jacketed CICC is as expected in the temperature range of 40 to $50 \mathrm{~K}$. The uncertainty on the critical current indicated by the error bars resulted from a change in temperature during the measurement runs in combination with small differences in starting temperature of each run. The Cu-jacketed CICC has a larger variance in starting temperature as the temperature of this conductor was difficult to regulate precisely due to the combination of the two cooling channels in this conductor (Figure 5.19), each supplying helium with a different temperature. The n-value of the SS-jacketed CICC is $14 \pm 3$ in the temperature range of 40 to $60 \mathrm{~K}$, similar to the n-value of $14 \pm 1$ measured for the first demonstrator CORC CICC at a temperature of $77 \mathrm{~K}$ and described above in Section 5.3. It was not possible to determine the n-value for the SS-jacketed CICC at temperatures lower than $40 \mathrm{~K}$, since the superconducting transition of this conductor could not be reached. The n-value of the Cu-jacketed CICC is significantly lower, with a value of $5 \pm 1$ in the temperature range of 40 to $60 \mathrm{~K}$, a clear indication of a very early onset of current sharing between CORC strands. A slightly higher n-value is measured at temperatures below $20 \mathrm{~K}$. No significant change is observed in critical currents and n-values of both conductors after the thermal cycle to room temperature between the measurement campaigns.
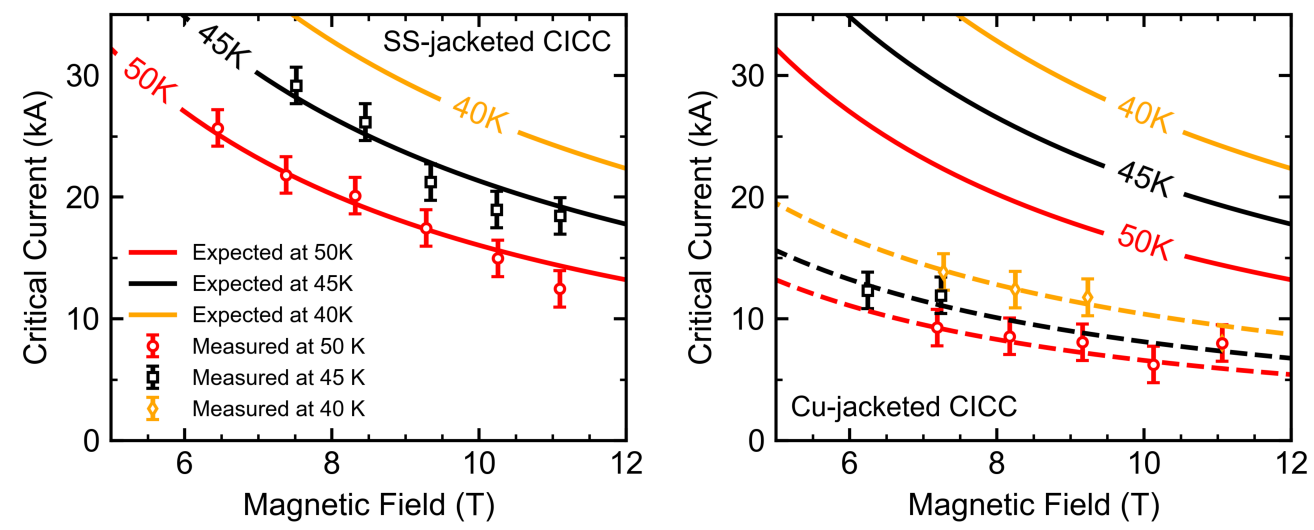

Figure 5.26: Average measured critical current (markers) of the SS-jacketed CORC CICC (left) and the Cu-jacketed CORC CICC (right) as function of total magnetic field. The SS-jacketed CICC demonstrates good correspondence to the expected critical current values (solid lines). The Cu-jacketed CICC does not. The small uncertainty of the measured in critical current values results from the fact that the temperature could not be kept stable at exactly the desired temperature and slightly increased during the measurement runs as the joint terminals heated the forced-flow helium gas. The temperature at $I_{c}$ is within $1 \mathrm{~K}$ of the desired temperature for the stainless steel CICC and within $2 \mathrm{~K}$ for the copper CICC. 


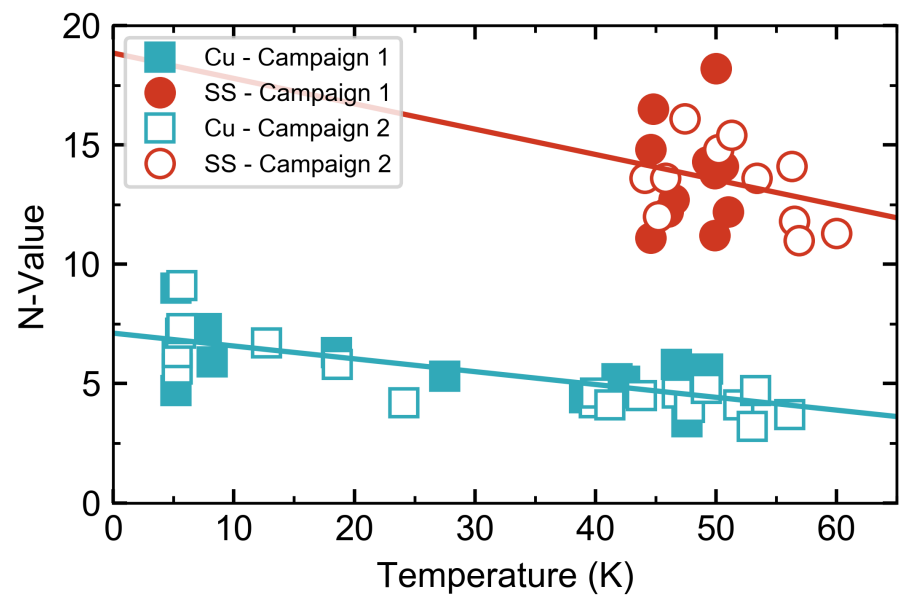

Figure 5.27: Measured n-values of both conductors before and after refurbishing of the bottom joint. The $n$-value of the CICC with the stainless steel jacket is $14 \pm 3$ in the temperature range of 40 to $60 \mathrm{~K}$. At temperatures lower than $40 \mathrm{~K}$ it was not possible to fit the n-value with a high accuracy since the superconducting transition of this conductor could not be reached. The n-value of the CICC with the copper jacket is substantially lower, with a value of $7 \pm 3$ at a temperature range of 5 to $40 \mathrm{~K}$ and $5 \pm 1$ at the temperature range of 40 to $60 \mathrm{~K}$.

\section{Feasibility of Conduction Cooling}

Two scenarios were tested to assess the efficiency of conduction cooling for CORC CableIn-Conduit Conductors. In the first scenario, the flow through cooling channel 2 (see Figure 5.19) was stopped completely, which results in internal forced-flow cooling of the SS-jacketed CORC CICC and external conduction cooling of Cu-jacketed CORC CICC via the cooling line integrated in its jacket. Current was ramped to $35 \mathrm{kA}$ in an external magnetic field of $10.9 \mathrm{~T}$. The initial temperature of both conductors was $5 \mathrm{~K}$ and it stabilized at approximately $10 \mathrm{~K} 500$ seconds after ramping was finished. The Cu-Jacket CORC CICC is close to it's critical current at the current $35 \mathrm{kA}$ at $10 \mathrm{~K}$. Stable operation of both conductors is possible even near and above the conductors critical current, as illustrated in Figure 5.28.

In the second scenario the flow through cooling channel 1 was stopped completely, which resulted in internal forced-flow cooling of the Cu-jacketed CORC CICC and conduction cooling of the SS-jacketed CORC CICC only through thermal conduction via the bottom joint that connected the two conductors and the limited amount of copper in- and around the CORC cable bundle. The temperature of the SS-jacketed CORC CICC started at $10 \mathrm{~K}$ and the temperature of the Cu-jacketed CORC CICC at $5 \mathrm{~K}$. Current was ramped up to $50 \mathrm{kA}$ and remained at this level for 300 seconds in an external magnetic field of $8 \mathrm{~T}$. The temperature of the $\mathrm{Cu}$-jacketed CORC CICC stabilized at $9 \mathrm{~K}$ and the temperature of the SS-jacketed CORC CICC stabilized around $14 \mathrm{~K}$. A change in sample current had a rather large influence on the temperature of the SS-jacketed CICC conductor; especially ramping 

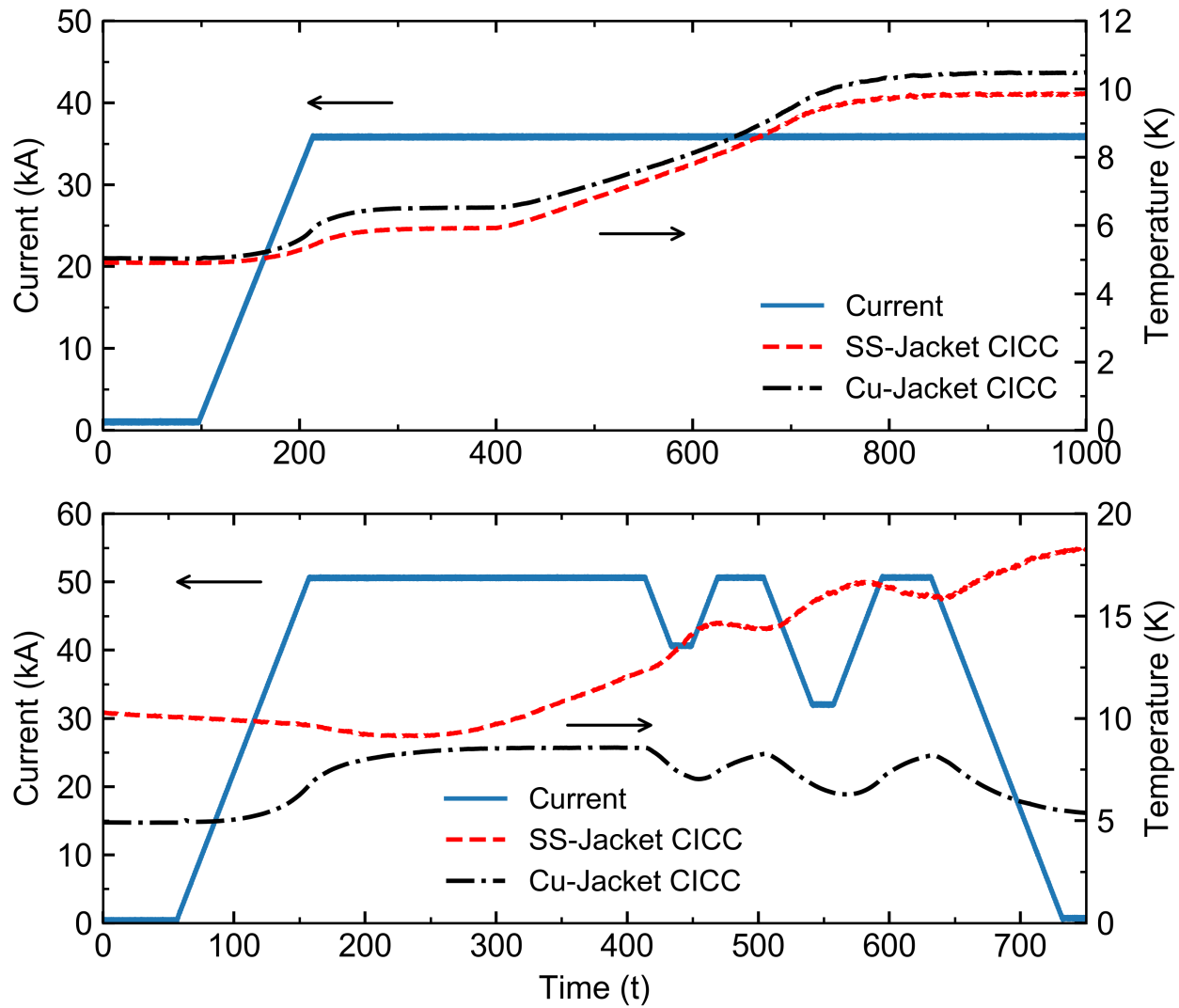

Figure 5.28: Current (solid blue line) and temperature evolution (dashed lines) in the two CORC CIC-Conductors in two scenario's. Scenario 1 (top) only has cooling through cooling channel 1 that allows helium forced-flow cooling of the SS-jacketed CICC and conduction cooling of the Cu-jacketed CICC via its jacket. Scenario 2 (bottom) has only forced-flow cooling through the Cu-jacketed CICC and has conduction cooling of the SS-jacketed CICC via its bottom joint terminal. Scenario 1 is tested in a background field of $10.9 \mathrm{~T}$ and with a current of $35 \mathrm{kA}$, close to the critical current of the Cu-jacketed CICC; stable operation is found at $10 \mathrm{~K}$. Scenario 2 is at a background field of $8 \mathrm{~T}$ to allow for a higher current of $50 \mathrm{kA}$ and the temperature stabilizes at $9 \mathrm{~K}$ and $14 \mathrm{~K}$ for the Cu and SS-jacketed CICC, respectively.

down to lower current caused an increase in temperature of the SS-jacketed CICC due to AC-loss in the cable bundle and in the copper tube surrounding it, as can be observed in Figure 5.28. Once the excursion from 50 to $40 \mathrm{kA}$ and back is finished, the temperature of the SS-jacketed sample drops again towards $14 \mathrm{~K}$. During this measurement run energy was dissipated mainly in the joint terminals and, due to the connected heat-exchanger, heat from the outgoing warm helium was transferred to the incoming cold helium thereby slowly increasing the temperature of the samples. 
Both test scenario's demonstrated the feasibility of conduction cooling as well as the high stability of ReBCO conductors.

\section{AC-loss in the CORC CICCs}

The AC-loss of the SS-jacketed CORC CICC was measured in an external magnetic field of $2 \mathrm{~T}$ and an AC magnetic field of $\pm 0.2 \mathrm{~T}$. The frequency of the applied AC magnetic field is between 0.1 and $1 \mathrm{~Hz}$. The sample is at $5 \mathrm{~K}$ and carries no current during this test. The temperature is measured on the conductor's jacket in positions at either side of the region exposed to the AC-field. The AC-loss per cycle is calculated using the enthalpy difference of the gas:

$$
Q=\left[\left(H_{12}-H_{11}\right) \dot{m}\right] / f
$$

Here, $H_{11}$ and $H_{12}$ are the enthalpies of the helium gas before and after flowing through the exposed sample section, respectively, $\dot{m}$ is the mass-flow and $f$ is the frequency of the AC magnetic field. The inlet temperature drifted slightly upwards during the ACloss measurements. Presumably, this change in temperature was caused by high AC-loss in the conductor with the higher-conductivity copper jacket. The outlet temperature also increased steadily over time and had to be compensated for the drift of the inlet temperature. A schematic of the AC-loss setup is presented in Figure 5.29.

The measured AC-loss is shown in Figure 5.30. The loss scales linearly with frequency, indicating that mainly eddy-current or coupling losses are observed. Given the large copper tube present around the six-around-one cable, it is likely that the loss is dominated by eddy currents within this tube. The copper tube around the six-around-one CORC cable is composed of two half shells. It is probable that the AC-loss is higher if the shell is a solid copper cylinder. Nevertheless, a value of $6.9 \pm 0.7 \mathrm{~mJ} / \mathrm{cycle} / \mathrm{cm}^{3}$ is found at a frequency of $1 \mathrm{~Hz}$, which is more than 4 times lower than the measured AC-loss in conductors for the ITER experiment using the same experimental settings [110].

The conductor was not cycled mechanically and thermally before the AC tests were performed and is thus considered to be a 'virgin' conductor. In $\mathrm{Nb}_{3} \mathrm{Sn}$ conductors for ITER it is usually observed that the AC-loss first decreases with mechanical cycling [111] and later increases substantially again [112]. Unfortunately, due to limited measurement time it was not possible to test for similar behavior with the CORC Cable-In-Conduit Conductors.

The loss in the Cu-jacketed CICC could not be determined, since the loss levels were much larger and no stable temperature could be reached. The copper cross-sectional area of this conductor is almost 3 times larger than that of the conductor with the stainless steel jacket and eddy current loss increases accordingly. The exotic conduction cooling layout, with two different flow directions in the Cu-jacketed CICC, also means that the temperature gradient was unfit for quantifying the AC loss in this conductor. 


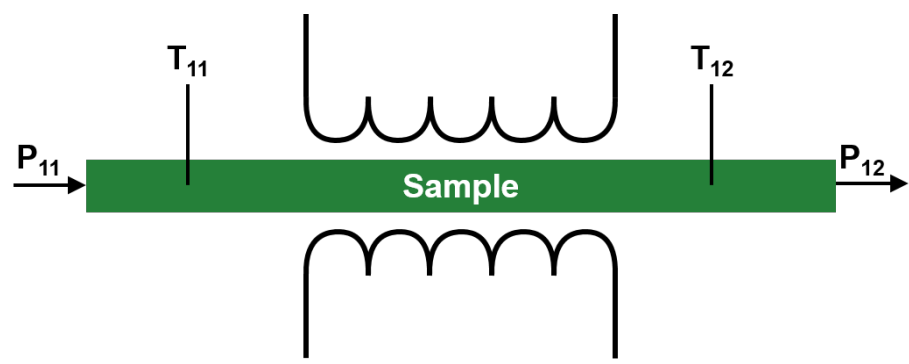

Figure 5.29: Schematic overview of the AC-loss setup. The heat generated by the AC-losses is transported along the conductor's length by the helium gas, which creates a temperature gradient over the conductor section which is exposed to the AC field. This temperature gradient and the pressure drop of the helium gas are used to calculated the AC-loss with the aid of Equation 5.1.

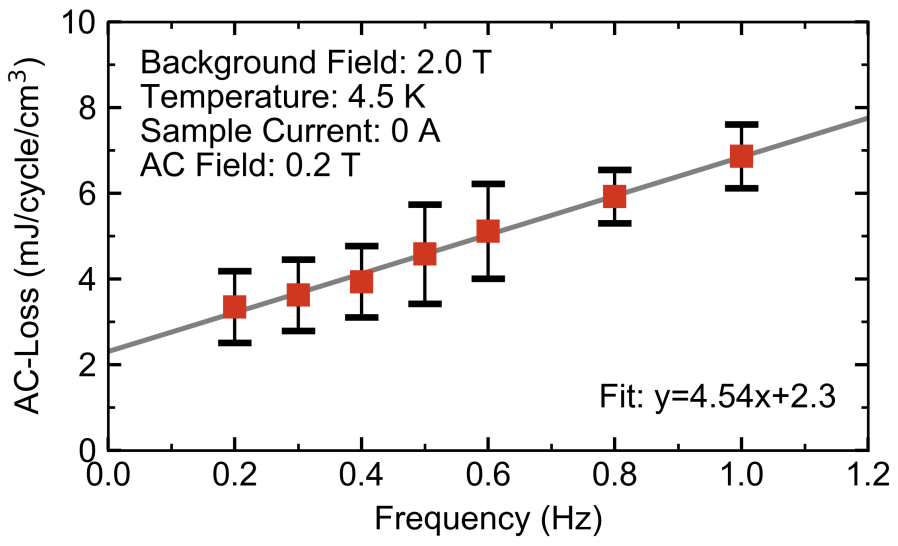

Figure 5.30: Measured AC-loss of the steel-jacketed CORC CIC-Conductor normalized to the cable volume including the copper tube that is mounted around the twisted strands. The AC-loss is proportional with frequency, indicating it to be dominated by eddy-current and coupling loss.

\section{Performance of the Joint Terminals}

The measured and predicted resistances of each joint terminal and the total resistance of the two individual samples for both measurement campaigns are presented in Figures 5.31 and 5.32 and in Table 5.3.

In the first cool-down campaign, a clear difference is found between the resistance of the 'top' terminals (connected to the HTS-adaptor of SULTAN) and the resistance of the bottom joint terminals (spliced together). The resistances of the top terminals are for both conductors close to their predicted values over the entire temperature range. The resistances of both bottom terminals are about twice the expected values. The splice that connects both samples together at the bottom of the sample-holder has a resistance of $17.1 \pm 0.5 \mathrm{n} \Omega$, much larger than expected. The difference in resistances between the 'top' and 'bottom' joint terminals can be explained by non-uniform current distribution due 
Table 5.3: Average measured and predicted joint terminal resistances for the top and bottom terminals of the two CORC CICCs for three temperature ranges using voltage tap pairs V11-V15, V1-V19, V12-V16 and $V 2-V 20$, as well as the measured and expected resistances of the splice that connects both samples at the bottom of the sample holder using voltage tap pair V17-V18.

\begin{tabular}{|c|c|c|c|c|c|c|}
\hline & Predicted & \multicolumn{2}{|c|}{ Campaign 1} & \multicolumn{2}{|c|}{ Campaign 2} & "Unit \\
\hline Cu-jacketed CICC & & Top & Bottom & Top & Bottom & \\
\hline $5-20 \mathrm{~K}$ & 1.9 & $2.4 \pm 0.1$ & $4.9 \pm 0.7$ & $2.1 \pm 0.4$ & $4.3 \pm 0.7$ & $\mathrm{n} \Omega$ \\
\hline $20-40 \mathrm{~K}$ & 3.5 & $3.0 \pm 0.7$ & $7.5 \pm 1.9$ & $3.2 \pm 0.6$ & $6.2 \pm 0.9$ & $\mathrm{n} \Omega$ \\
\hline $40-60 \mathrm{~K}$ & 6.0 & $5.7 \pm 2.3$ & $12.4 \pm 2.9$ & $4.5 \pm 0.7$ & $8.0 \pm 0.9$ & $\mathrm{n} \Omega$ \\
\hline SS-jacketed CICC & & Top & Bottom & Top & Bottom & \\
\hline $5-20 \mathrm{~K}$ & 1.9 & $2.2 \pm 0.2$ & $4.3 \pm 0.4$ & $2.1 \pm 0.1$ & $4.1 \pm 0.4$ & $\mathrm{n} \Omega$ \\
\hline $20-40 \mathrm{~K}$ & 3.5 & $2.7 \pm 0.4$ & $6.1 \pm 1.7$ & $3.0 \pm 0.8$ & $6.0 \pm 1.7$ & $\mathrm{n} \Omega$ \\
\hline $40-60 \mathrm{~K}$ & 6.0 & $5.4 \pm 2.5$ & $12.4 \pm 5.4$ & $5.6 \pm 2.0$ & $11.1 \pm 3.4$ & $\mathrm{n} \Omega$ \\
\hline $\begin{array}{l}\text { Bottom Splice } \\
5 \mathrm{~K}\end{array}$ & 2.2 & \multicolumn{2}{|c|}{$17.1 \pm 0.5$} & \multicolumn{2}{|c|}{$3.3 \pm 0.2$} & $\mathrm{n} \Omega$ \\
\hline
\end{tabular}
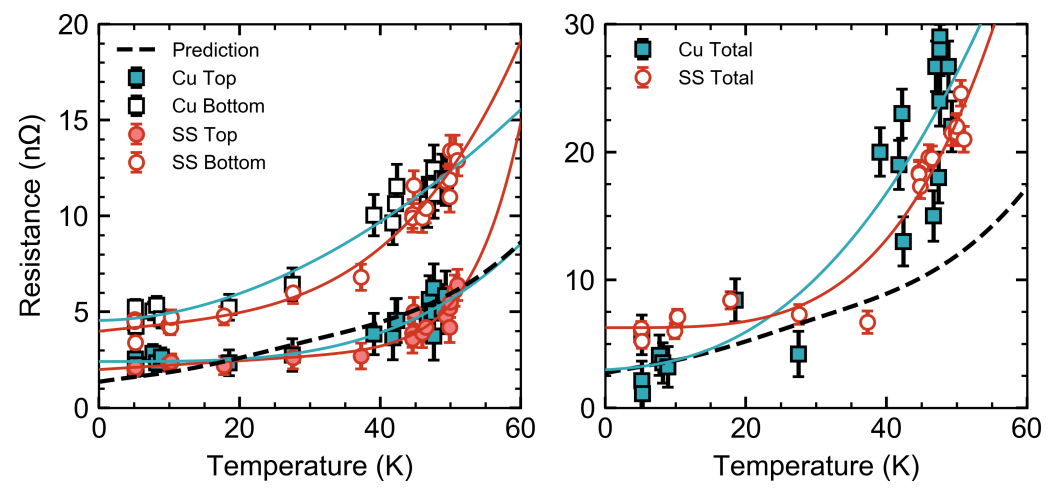

Figure 5.31: Left: the resistance of the top and bottom joint terminals of the Cu- and SS-jacketed CORC CICCs presented as function of temperature for the first measurement campaign. Symbols represent the measured data, the dashed line indicates the model prediction and the solid lines are the splines that serve as a guide for the eye. A clear difference is observed between the top and bottom joint terminals of both conductors. The top terminals have a significantly lower resistance than the bottom ones, indicating imperfect current distribution between strands. The resistance of the bottom terminals is 3 to $7 \mathrm{n} \Omega$ higher than expected, while the resistance of the top terminals is slightly lower than expected. Right: the total resistance over each sample is presented as function of temperature. For both conductors, this total resistance is higher than expected, but close to the sum of the resistances of both top and bottom terminals.

to the relatively bad splice between the bottom joint terminals of the two conductors. The total resistance of each conductor, measured from joint to joint, is some $15 \%$ larger than the sum of the resistance of their two joint terminals. Consequently some current redistribution appears to occur also in the conductor section between the joints. 

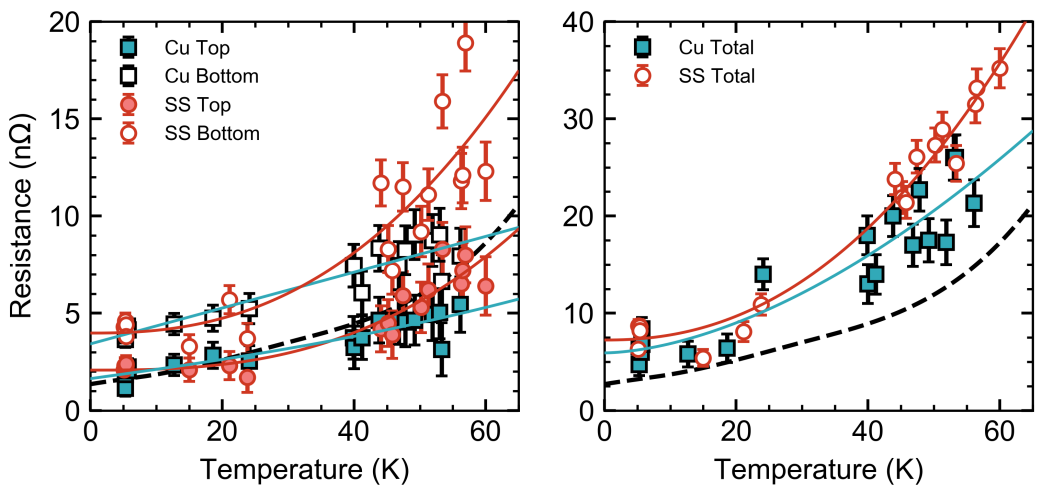

Figure 5.32: Left: the resistances of the top and bottom joint terminals of the Cu- and SS-jacketed CORC CICCs as function of temperature for the second test campaign, after the revision of the splice between the two bottom joint terminal. Only minor changes are observed in the resistance of the top joint terminals of both conductors. The resistance of the bottom joint terminal of the Cu-jacketed CICC decreased while the resistance of the bottom joint terminal of the SS-jacketed conductor did not change. Right: the total resistance over each sample as function of temperature. Minor changes are observed in the total resistance of Cu-jacketed CICC. A slight increase in total resistance is observed in the SS-jacketed CICC.

The bottom joint terminals of the two conductors are clamped together with indium as interface layer for the first campaign. This jointing method was successfully applied to the connection between the sample and the HTS adaptor of the SULTAN facility, but seems to have failed in this splice causing the large splice resistance of $17.1 \mathrm{n} \Omega$. The bottom joint terminals are in the stray field of the facility's dipole magnet and are subjected to large Lorentz forces pointing outwards. Insufficient tension can have caused slight opening of the clamped joint during operation. Therefore the splice of the bottom joint terminals was revised for the second test campaign. In the new splice, the two terminals were soldered with indium instead of just clamped. As a result, the splice resistance between the two joint terminals was reduced from $17.1 \pm 0.5 \mathrm{n} \Omega$ to $3.3 \pm 0.2 \mathrm{n} \Omega$ at $4.5 \mathrm{~K}$.

In the second measurement campaign, minimal change in the resistance of both 'top' terminals was observed after refurbishing the splice between the two CIC-Conductors. Both top terminals still have a resistances close to their predicted values. The resistance of the bottom terminal of the Cu-jacketed CICC is reduced by approximately $4 \mathrm{n} \Omega$ in the temperature region of 40 to $60 \mathrm{~K}$, but is still slightly above its expected value. No change is observed in the average joint resistance of the bottom terminal of the SS-jacketed CICC compared to its resistance during the first measurement campaign. However, a larger spread in joint resistance is measured. A slight decrease of 2 to $3 \mathrm{n} \Omega$ is observed in the total joint-to-joint resistance of the Cu-jacketed CICC between the two measurement campaigns, following the decrease in resistance of its bottom joint terminal. An increase of 2 to $3 \mathrm{n} \Omega$ is observed in the total joint-to-joint resistance of the SS-jacketed CICC. The total resistance over the two samples is for both samples on average 35\% higher than the sum of the resistance of the two joint terminals of each conductor. Therefore, it is likely 
that the current distribution is less homogeneous after the refurbishment once the large resistive barrier between the two conductors has been removed and defects in either the joints or, more likely, the degraded conductor become an important factor.

The resistance for both top joint terminals, and in the second measurement campaign also the bottom joint terminal of the Cu-jacketed CICC, are very close to the expected joint resistances. Nevertheless, further improvements are required as the total resistance over the samples is still larger than the sum of the individual joint terminals, an indication that the current distribution is still imperfect. The two bottom joint terminals that make a 'praying hands' type splice perform worse than the top joint terminals that have a 'shaking hands' type of splice. A similar effect is observed in joint performance during the test of the first demonstrator CORC Cable-In-Conduit Conductor at CERN (see Section 5.3.3). The type of splice has influence on the joint resistance and current distribution, especially during current ramping.

\subsubsection{Extracted Single-Strand and Single Tape Analyses}

During the characterization test of the two CORC CICC conductors, the Cu-jacketed CORC CICC performed far worse than expected over the entire temperature and magnet field ranges. Therefore, this sample was examined in detail. The jacket of this conductor was opened, which allowed for inspection of the individual strands in an attempt to identify the position and nature of the failure.

Clear indentations of the strands were observed on both halves of the copper jacket; the deepest indentations were present in the half to which the largest Lorentz forces were directed, smaller indentations were present on the other, as shown in Figure 5.33. Some gaps of a few $\mathrm{mm}$ were found between CORC strands, primarily in the cable section that was located outside the high-field region. These were not present (or less pronounced) before the jacketing, which indicates movement of CORC strands occurred during the tests. No clear indication of failure could be found by further visual inspection of the outer surface of the cable only.

A more in-depth analysis was performed on the single CORC strands in the six-aroundone cable at Advanced Conductor Technologies. Voltage taps were attached to various positions on the single CORC strands to monitor single strand behavior. Single strand instrumentation was not implemented during the test in the SULTAN facility, as it would over-complicate the experimental setup to assure leak tightness of the samples. The CORC CICC, without the jacket, was tested at a temperature of $76 \mathrm{~K}$ up to a current of $13 \mathrm{kA}$. The joint resistance was measured by voltage tap pairs from the copper joint terminals for each of the CORC strands. The joint terminals shows a resistance of $50 \mathrm{n} \Omega$ during the test, approximately 2.5 times higher than the measured resistance at $50 \mathrm{~K}$. Two adjacent strands are observed to perform much worse than the others, as shown in Figure 5.34. Strands 2 and 3 started the transition at a current of around $6 \mathrm{kA}$, strands 1, 4 and 6 at a slightly higher current of around 8 to $9 \mathrm{kA}$ and stand 5 at a current of $11 \mathrm{kA}$. These results constitute another clear sign of either severe degradation of the CORC strands or very non-uniform current distribution between strands initiated in the joint terminals. 


\subsection{TWO NEW CORC CICC SAMPLES FOR MAGNETS AIMING AT FUSION EXPERIMENTS AND PARTICLE DETECTORS}

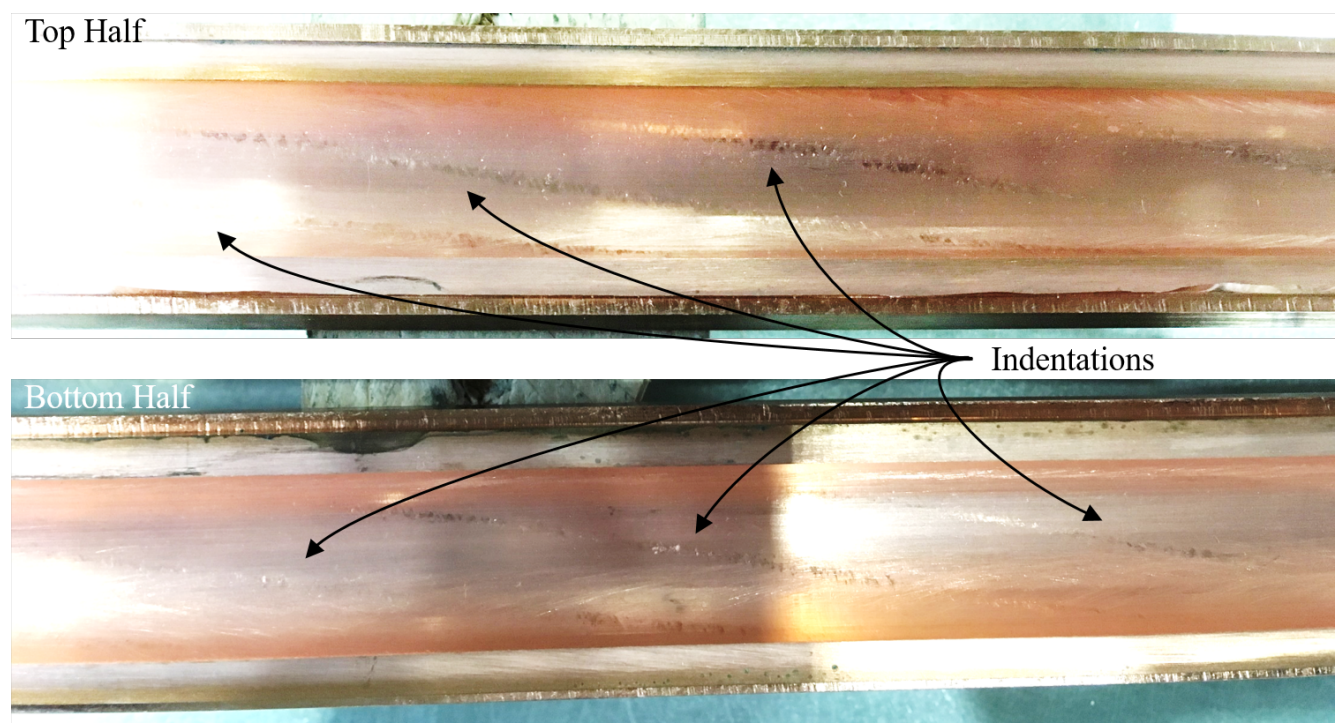

Figure 5.33: Picture of the top and bottom halves of the copper jacket with clear indentations of the CORC strands visible. The indentations are deeper on the top half of the jacket as the Lorentz forces pointing in that direction were larger than those pointing towards the bottom half of the jacket.

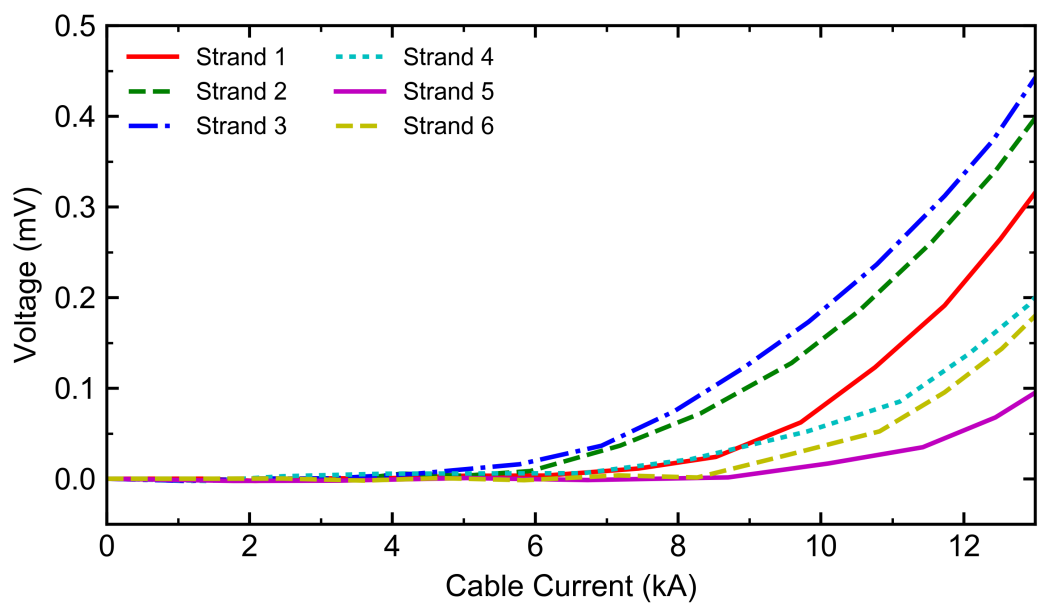

Figure 5.34: Voltage over the strands as function of overall cable current at $76 \mathrm{~K}$ measured at and reported by ACT [113].

\section{Single Strand Analyses}

The top joint terminal was removed from the six-around-one cable bundle and singletube terminals were attached to the ends of each individual CORC strand, as displayed in 
Figure 5.35. Current was injected consecutively into each of the single strands while the conductor was submerged in liquid nitrogen to characterize the single strand performance and to localize any defects. E(I) curves obtained from these single-strand measurements, as shown in Figure 5.36, are similar to the earlier measurement of six-around-one cable bundle, which proves that the measured poor CICC behavior is a result of severe strand degradation and not due to inhomogeneous current distribution effects. Especially two CORC strands are severely degraded and retain only $20 \%$ to $30 \%$ of the expected $\mathrm{I}_{c}$. The other four strands showed less, but still significant degradation and had an $\mathrm{I}_{c}$ retention ranging from $40 \%$ to $60 \%$.

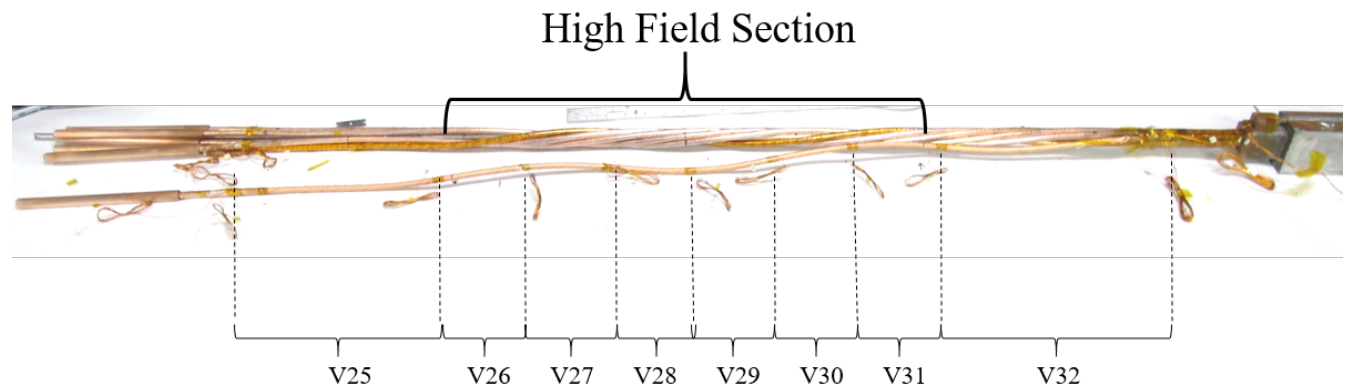

Figure 5.35: Picture of the six-around-one CORC bundle extracted from the Cu-jacketed CICC with separate joint terminals attached to the ends of each of the six CORC strands. Voltage taps were attached to the strands to measure their individual performance and to localize the point of failure. The picture is courtesy of ACT.
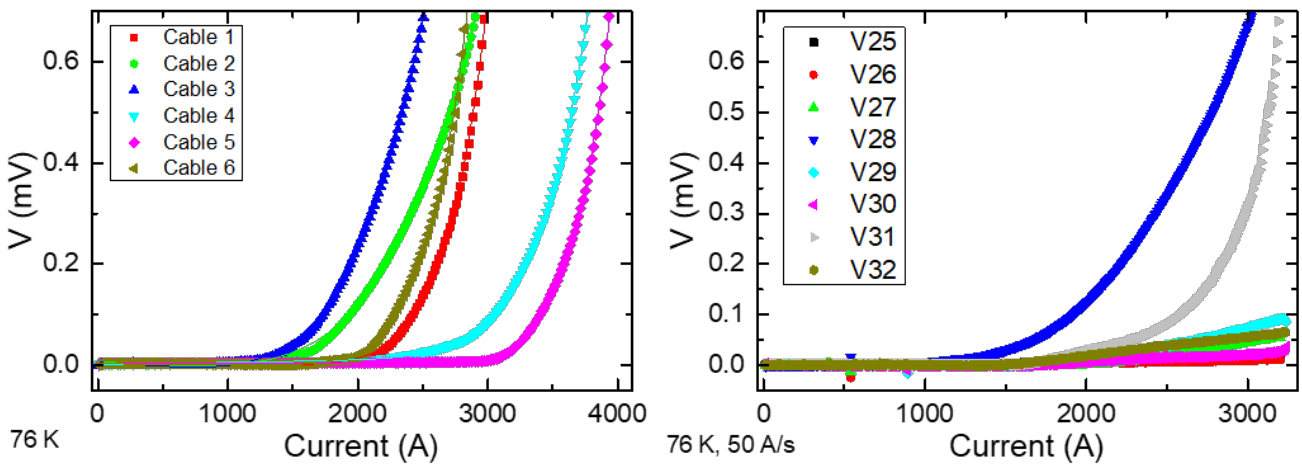

Figure 5.36: Left: V(I) curves of the individually tested strands at $76 \mathrm{~K}$ measured at and reported by ACT [113]. Right: V(I) curves of eight voltage tap pairs attached to the CORC strand nr. 3, which demonstrate very local degradation within the sections of voltage tap pair V28 and V31.

The strand sections that experienced the most severe degradation are located within voltage tap pairs V28 and V31, as shown in Figure 5.36, both in the conductor section that was in the high-field region of SULTAN. This indicates that the degradation was likely caused by the electro-magnetic load during the test. Closer visual inspection of 


\subsection{TWO NEW CORC CICC SAMPLES FOR MAGNETS AIMING AT FUSION EXPERIMENTS AND PARTICLE DETECTORS}

the worst performing section showed signs of damage resulting in a 'teardrop'-shaped conductor cross-section, which means the conductor irreversibly deformed under the large EM-load. It should be noted that the cable comprised a full twist pitch within the highfield region, therefore it might be expected that all strands would experience similar levels of degradation. However, this is not observed and the reason for this is uncertain.

\section{Single Tape Analyses}

A $10 \mathrm{~cm}$ long section was taken from the most severely degraded strand $\mathrm{nr} .3$ and its tapes were extracted for even more detailed $\mathrm{I}_{c}$ measurements. Only 2 out of the 42 tapes carried current close to their original critical current (expected to be $145 \mathrm{~A}$ ), all others were moderately to severely degraded. The most severe degradation occurred to the tapes located in the most inner 8 layers, which had only 0 to $20 \% \mathrm{I}_{c}$ retention, followed by 6 layers with an average of $50 \% \mathrm{I}_{c}$ retention, as presented in Figure 5.38. The overall tape $\mathrm{I}_{c}$ of this cable adds up to $1858 \mathrm{~A}$, which is $26 \%$ strand $\mathrm{I}_{c}$ retention. Visual inspection of the tapes showed the same type of deformation, as shown in Figure 5.37, which was most pronounced on the tapes situated closest to the strand's core.

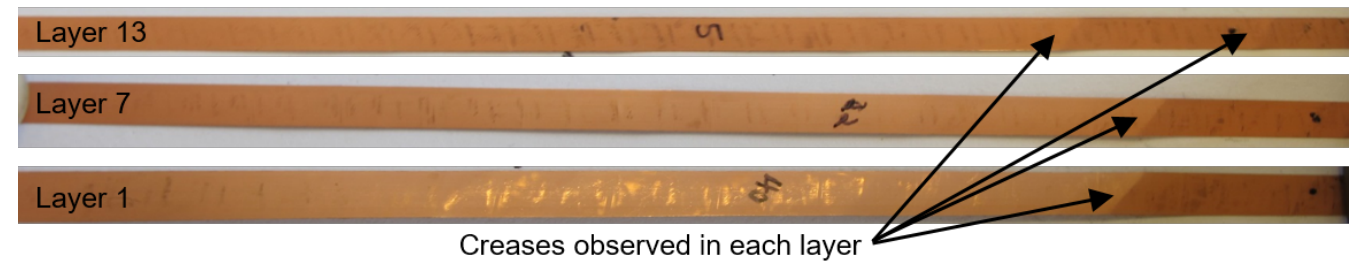

Figure 5.37: Observed creases in tapes from each layer corresponding to the strand position that showed the most severe degradation [113].
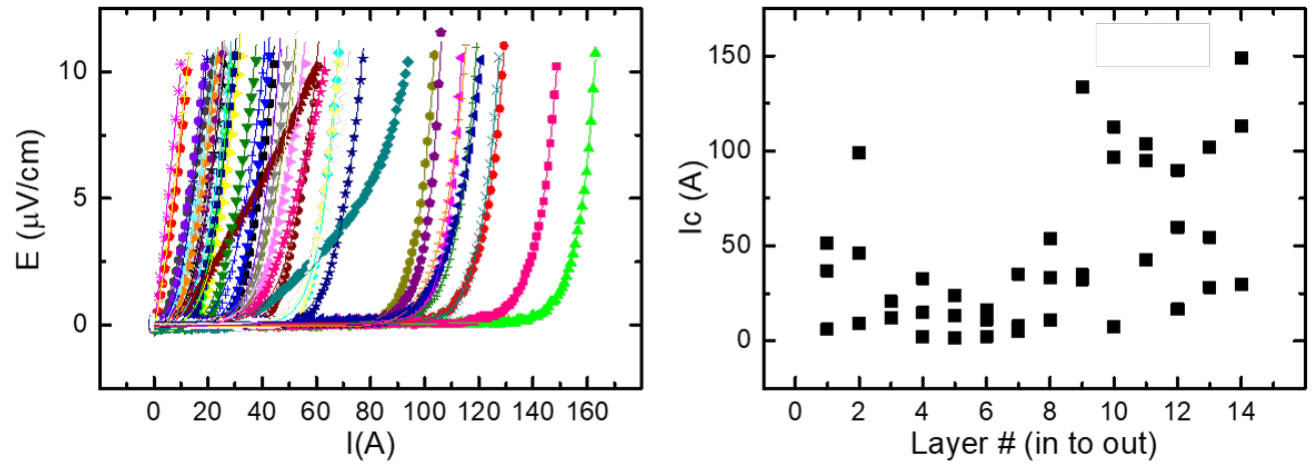

Figure 5.38: Left: E(I) curves of the extracted tapes at $76 \mathrm{~K}$ measured at and reported by ACT [113]. Right: measured critical current as function of layer position within the CORC strand. Tapes located closer to the strand's core suffered larger degradation compared to tapes located in the more outer situated layers. 


\subsubsection{Plausible Cause of the Observed Degradation}

A feasible explanation for the large reduction of the tape's critical current is the Lorentz force during the test causing a 'pinching' effect on the CORC strands, instead of a uniform transverse load, as schematically illustrated in Figure 5.39. Such unevenly distributed EM-load can push the local strain in the tapes over their critical limit. This effect would be more pronounced in tapes closer the strand's core, since these are already heavily strained due to their small bending radius. This scenario is supported by the $\mathrm{I}_{c}$ measurement on the tapes extracted from the most degraded strand (Figure 5.38).

Only the Cu-jacketed CICC showed severe degradation during the test in the SULTAN facility. The SS-jacketed CICC did not provide any indication that it suffered from a similar defect. The difference between the two conductors is primarily in the layout and cabling parameters of the CORC strands that were used. The CORC strands for the $\mathrm{Cu}$ jacketed CICC have a solid copper core of $4 \mathrm{~mm}$ in diameter, extended to $5 \mathrm{~mm}$ by copper tapes, whereas the strands for the SS-jacketed CICC have a solid copper core of $5 \mathrm{~mm}$ in diameter. It appears that the thinner core in the strands of the Cu-jacketed CICC turned out to have a negative effect on the strands' resilience against the large electro-magnetic loads. Therefore the root cause of the degradation can traced back to a cabling parameter unique to the preparation of the CORC strands for the Cu-jacketed CICC. Also, a larger tolerance between the six-around-one cable bundle and jacket allowed more movement of the CORC strands in the Cu-jacketed CICC compared to the SS-jacketed one under high EM-load conditions. This could have had a negative effect on how the transverse forces were handled by the CORC strands. The conclusion is that the combination of the CORC strand internal layout, tape winding tension and looseness of the cable bundle in the CICC jacket all likely have played a large role in the eventual degradation of the $\mathrm{Cu}$-jacketed CICC. This observation constitutes important knowledge gained from the test and can be used to further optimize strand and cable manufacturing.
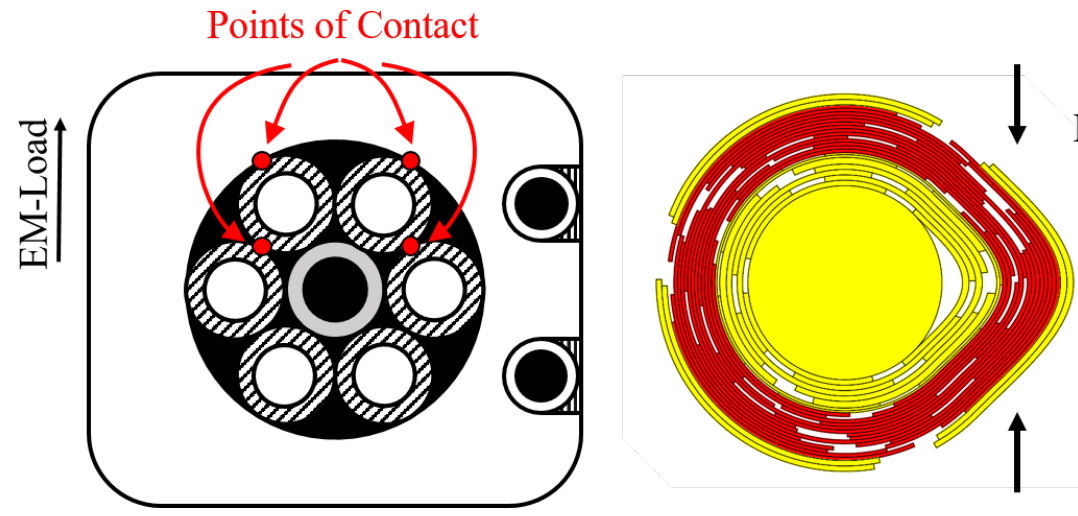

Pinching Deformation

Figure 5.39: Illustration of the pinching effect that plausibly has caused the measured strand degradation. The points of contact and the direction of the large EM-loads were not centered relative to the strands core. This can cause a 'pinching' effect on the tapes as the forces are transferred by the tapes instead of the strand's core, thereby pushing the strain within the affected tapes over its critical limit. 


\subsubsection{Discussion on Future Conductor and Joint Improvements}

The Cu-jacketed CICC sustained severe degradation during the test in the SULTAN facility. The primary cause of the degradation is a pinching effect on the strands, introduced by Lorentz forces on the strands causing movement and deformation, due to a combination of strand internal layout, winding tension and looseness of the cable bundle. Strand preparation and cable parameters therefore need to be adjusted to prevent such defect from re-occurring. A new CORC CIC-Conductor is under development to replace the degraded $\mathrm{Cu}$-jacketed CICC. The new conductor features a further optimized six-aroundone CORC cable bundle with a copper jacket. The strand has a central core of $5.4 \mathrm{~mm}$, slightly higher than the core in the strands of the current SS-jacketed CICC. The sixaround-one cable bundle will be twisted more tightly to reduce the spacing between strands and will have a closer fit in the jacket to prevent strand movement under Lorentz forces.

The joint terminals performed close to expectations. Nevertheless, still some signs of imperfect current sharing between CORC strands were observed. Therefore, in parallel with the design of the new CORC CICC, a new and improved joint terminal is under development allowing improved current sharing among CORC strands and further reducing the overall joint resistance. In the current joint terminal design all strands are inserted into a shared bore in a large copper terminal, which is thereafter filled with $\mathrm{SnPb}$ solder material. In the new joint, indium will be used to reduce the joint contact resistances further. Also, pure indium has a lower mechanical yield strength and stiffness compared to $\mathrm{SnPb}$, which can alleviate stress concentrations inside the terminals due to the thermal shrinkage mismatch between the solder and the ReBCO tapes.

\subsubsection{Conclusion}

Two new ReBCO CORC Cable-In-Conduit Conductors were designed, prepared and tested with an unprecedented current-carrying capacity. The conductors feature a new design that allows for a combination of external conduction cooling and/or internal forced-flow gas cooling. The conductor samples have a length of $2.7 \mathrm{~m}$, including joint terminals, and are rated for $80 \mathrm{kA}$ at $12 \mathrm{~T}$ and $4.5 \mathrm{~K}$. The conductors differ from each-other in the choice of jacket material and of strand cabling parameters. One has a copper jacket to optimize conduction cooling, while the other has a stainless steel jacket that is able to cope with the considerable electro-magnetic loads which are common to conductors in large tokamak magnets.

The two Cable-In-Conduit Conductors were tested together in a 'hairpin' combination connected electrically in series in the SULTAN facility of EPFL in Villigen, Switzerland, in a temperature range from 5 to $60 \mathrm{~K}$ and a magnetic field range from 5 to $10.9 \mathrm{~T}$.

The Cu-jacketed CICC showed severe degradation, with a measured critical current of only $30 \%$ to $40 \%$ of it's expected value throughout the entire temperature and magnetic field range. This measured critical current is of $37 \pm 4 \mathrm{kA}$ at $5 \mathrm{~K}$ and $10.9 \mathrm{~T}$ applied magnetic field. 
The SS-jacketed CICC, on the other hand, has a measured $\mathrm{I}_{c}$ value that is close to the expected critical current in the temperature range of 40 to $60 \mathrm{~K}$. The critical current of this conductor could not be determined at temperatures lower than $40 \mathrm{~K}$, since the neighboring Cu-jacketed CICC showed thermal runaway behavior before the SS-jacketed CICC reach the onset of the superconducting to normal transition.

The n-value of the Cu-jacketed CICC is low, with a value of $5 \pm 1$. The n-value of the SSjacketed CICC is $14 \pm 3$, which is similar to the n-value of the first CORC Cable-In-Conduit Conductor sample characterized at CERN.

Three out of the four joint terminals show resistance values close to the expected ones, while one joint terminal has a resistance that is several $\mathrm{n} \Omega$ higher than expected. The total resistance, measured from joint- to joint terminal, for both conductors is $35 \%$ higher than the sum of the two single joint terminal, indicating that some current is still redistributed outside the joint terminal. This is possibly directly related with the degradation of the $\mathrm{Cu}$ jacketed conductor. Further improvement of the cable and the joint terminal technology is therefore still required.

The strands from the degraded Cu-jacketed CICC were extracted and characterized to identify the failure mode. Two CORC strands showed severe degradation and had only $20 \%$ to $30 \% \mathrm{I}_{c}$ retention. The other four strands showed less, but still sizable degradation and had an $\mathrm{I}_{c}$ retention ranging from $40 \%$ to $60 \%$. The degradation was narrowed down to the conductor section positioned in the high-field region of SULTAN. Visual inspection and analyses of single tapes extracted from the worst performing strand revealed that the lowest $\mathrm{I}_{c}$ retention is situated in the inner most ReBCO layers. The primary cause of the degradation appears to be a pinching effect on the strands that occurred due to the Lorentz force in high magnetic field, which was traced back to non-optimal cabling parameters unique to the strands in the $\mathrm{Cu}$-jacketed CICC. The observed degradation mode is not intrinsic to the CICC design and optimization of strand cabling parameters and a tighter fit of the cable bundle in the jacket in all likelihood will avoid such future problems.

A new Cu-jacketed CICC is under development, featuring improved CORC strands and joint terminals. This new conductor will be tested in the autumn of 2018. 


\subsection{CORC CICC Bus Bars}

Bus lines that feed large currents of many kilo-amperes to large magnet systems are commonly composed of conventional copper or aluminum conducting materials. These materials are practical in use, however they have the disadvantage that the power converters need to be relatively close to the magnets to limit the operating voltage and ohmic loss. CERN is developing $\mathrm{MgB}_{2}$ superconducting links rated for $150 \mathrm{kA}$ to replace current copper conducting that feed current to the magnets systems of the Large Hadron Collider (LHC) [9]. These superconducting links allow placement of the power-converters further away from the LHC machine which excludes radiation damage to the devices, allows more practical access for maintenance and the switch from normal conducting to superconducting materials decreases the voltage drop over the bus bars and thus the DC power loss.

The Future Circular Collider (FCC) is a proposed next collider after the Large Hadron Collider. The FCC features a $100 \mathrm{~km}$ long circular collider up to $350 \mathrm{~m}$ underground. The detector magnets in the current design feature operating currents in the order of 30 to $80 \mathrm{kA}$ and the dipole and quadrupole magnets inside the ring feature currents of 12 to $16 \mathrm{kA}$. Maintenance and operation of the cryogenic plant and the power converter of the detector magnets are more practical when located on the surface. Therefore bus bars of some $400 \mathrm{~m}$ in length are required to bring current down to the detector magnets, thereby vastly increasing the voltage and thereby power requirements of the power converter.

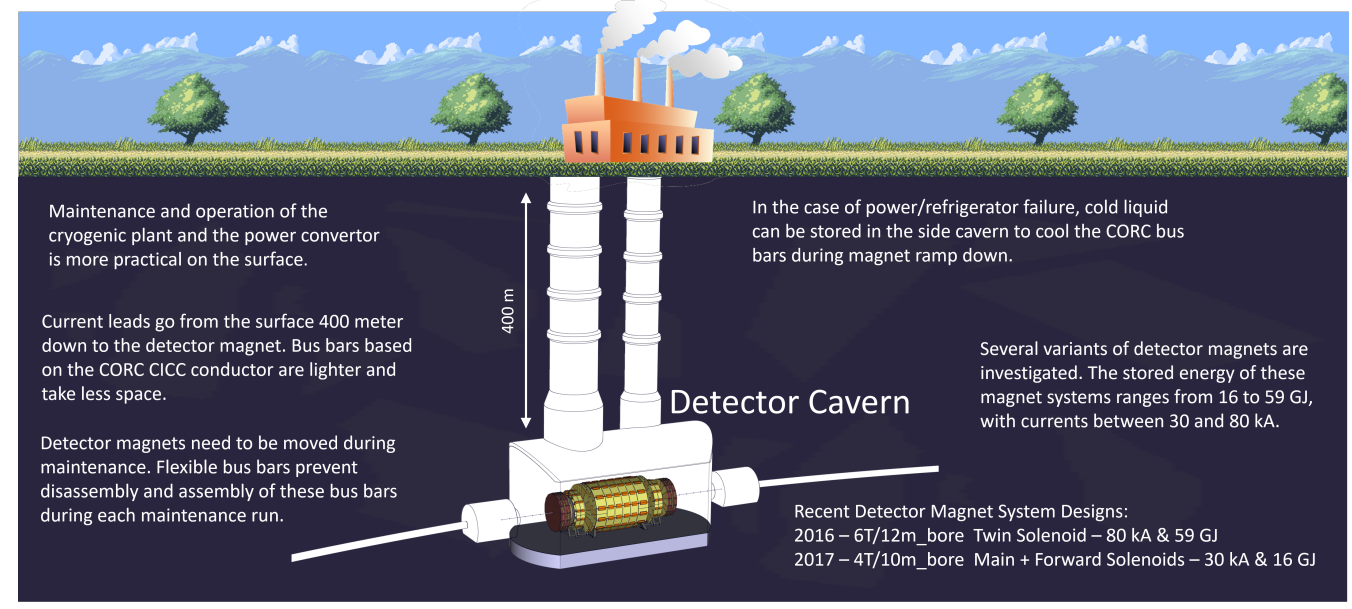

Figure 5.40: Illustration of a future detector magnet and the requirements for its bus lines that feed current from the surface down to the detector cavern. 


\section{Benefits and Requirements of Superconducting Bus Bars}

Various designs of magnet systems for the future detectors are under development. The first design, dating back to 2016, features an actively shielded twin solenoid design operating at $80 \mathrm{kA}$ with a total stored energy of 59 GJ [114]. A second design in 2017 features a less challenging magnet system operating at $30 \mathrm{kA}$ with a stored energy of 16 GJ [115]. Table 5.4 presents operational details on the current bus bars of the ATLAS detector magnets, details of the requirements for the future twin solenoid detector magnet for both normal and superconducting bus lines. Superconducting bus bars, i.e. based on ReBCO coated conductor technology, can realize a reduction in operating voltage, volume and mass compared to pure aluminium bus bars. However, superconducting bus-lines do require a more complex cooling, maintenance and safety infrastructure compared to conventional bus lines:

- Superconducting bus lines require cooling to a temperature below their currentsharing temperature. Cooling can be established via cold gas returning from the magnet system to the magnet's liquefier. In the case of cryogenic failure back-up cooling of the CORC bus lines can be established using helium stored in the side cavern for magnet ramp down.

- Maintenance work on the detector commonly requires opening and closing of the magnet system without detaching the bus lines and therefore the bus lines require flexibility while cold.

- ReBCO bus lines have to survive a quench in the detector magnets and a quench in the bus bars themselves. Sufficient stabilizer material parallel to the superconductor is required to assure hot spot temperatures below the limit of degradation during a quench event.

The advantage of omni-directional bending in a superconductor is unique to the ReBCO CORC conductor for the operating temperature range of 20 to $80 \mathrm{~K}$, which in combination with the high thermal and electrical stability of ReBCO makes CORC conductors a very suitable candidate for use in a superconducting bus bar.

Table 5.4: Parameters of bus bars of the magnet system of the ATLAS detector magnets and the future potential normal en superconducting bus bars of an $80 \mathrm{kA}$ detector magnet.

\begin{tabular}{llll}
\hline \hline & ATLAS Bus Bars & Future Normal & Future SC \\
& Bus Bars & Bus Bars & Bus Bars \\
\hline Current (kA) & 20 & 80 & 80 \\
Material & Aluminium & Aluminium & ReBCO CORC \\
Length (m) & 300 & 1000 & 1000 \\
Cross-section $\left(\mathrm{cm}^{2}\right)$ & 400 & 1600 & 200 \\
$\mathrm{~J}_{\text {stabilizer }}\left(\mathrm{A} / \mathrm{mm}^{2}\right)$ & 0.5 & 0.5 & 10 \\
Mass (Tonnes) & 33 & 430 & 80 \\
Voltage @ Operation $(\mathrm{V})$ & 8 & 28 & - \\
Power @ Operation $(\mathrm{kW})$ & 160 & 2240 & - \\
\hline \hline
\end{tabular}




\section{Design of a CORC Six-Around-One Flexible Bus Bar}

The ReBCO CORC conductor is a flexible conductor that can carry large currents as a single conductor or as a six-around-one cable bundle (demonstrated in Sections 5.3 and 5.4) or any other convenient layout. The six-around-one cable bundle is able to carry the current required in both the twin-solenoid and the forward-solenoid detector magnet designs when a similar CORC strand is used as in the latest two CORC Cable-In-Conduit Conductors. The bus bars are in the stray field of maximum $1 \mathrm{~T}$. The six-around-one cable bundle is expected to carry the $80 \mathrm{kA}$ required for the twin solenoid magnet design at a temperature of $55 \mathrm{~K}$, while currents of $30 \mathrm{kA}$ already can be reached at a temperature of $70 \mathrm{~K}$ in a background magnetic field of $1 \mathrm{~T}$.

Cooling of the conductor can be established by either conduction cooling via integrated cooling lines within or around the CORC bundle. Additional copper stabilizer is added in parallel to the CORC cable bundle to protect it in the case of a quench or cryogenic failure. A 3D-generated representation of a preliminary design of the CORC six-around-one bus bar is presented in Figure 5.41.

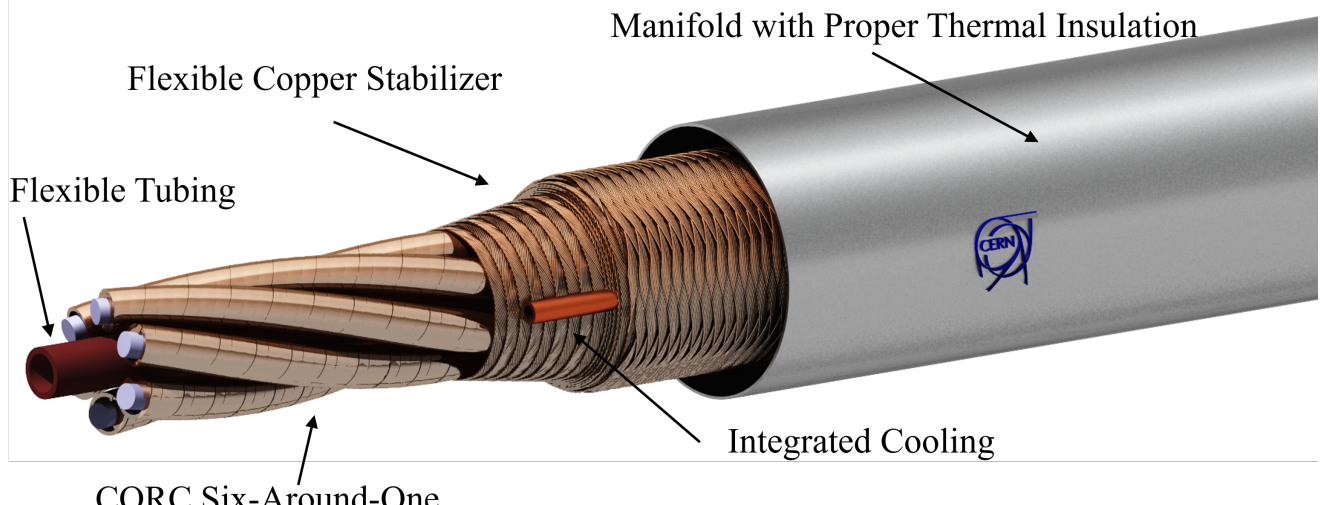

Figure 5.41: 3D-generated image representing a preliminary design of the CORC six-around-one bus bar. The design features the six-around-one CORC bundle, wrapped in several layers of flexible copper braid wires with integrated cooling tubes for helium flow.

The required amount of copper stabilizer depends on the maximum allowable hot spot temperature of the bus bar. Adding more stabilizer reduces the normal resistance of the bus bar and increases its thermal capacity. A plausible operating scenario is a quench in only one bus bar, after which current has to be extracted from the magnet using its dump resistor. The maximum hot spot temperature of the bus bars are calculated using the parameters of the twin solenoid detector magnet, which uses a $20 \mathrm{~m} \Omega$ dump resistor in its current design, as shown in Figure 5.42. A hot spot temperature of $150 \mathrm{~K}$ is reached with a stabilizer current density of $10 \mathrm{~A} / \mathrm{mm}^{2}$, which is only a temperature rise of $100 \mathrm{~K}$. Minimal difference in current extraction time is seen between the various current density scenarios as most of the stored energy is dissipated in the dump resistor of the magnet's 
protection system. The second detector magnet design, with an operating current of $30 \mathrm{kA}$ and a stored energy of $16 \mathrm{GJ}$, can have bus bars with much higher copper current density as there is less energy to be dissipated. In this case a similar hot spot temperature of $150 \mathrm{~K}$ is reached with a stabilizer current density of $15 \mathrm{~A} / \mathrm{mm}^{2}$.
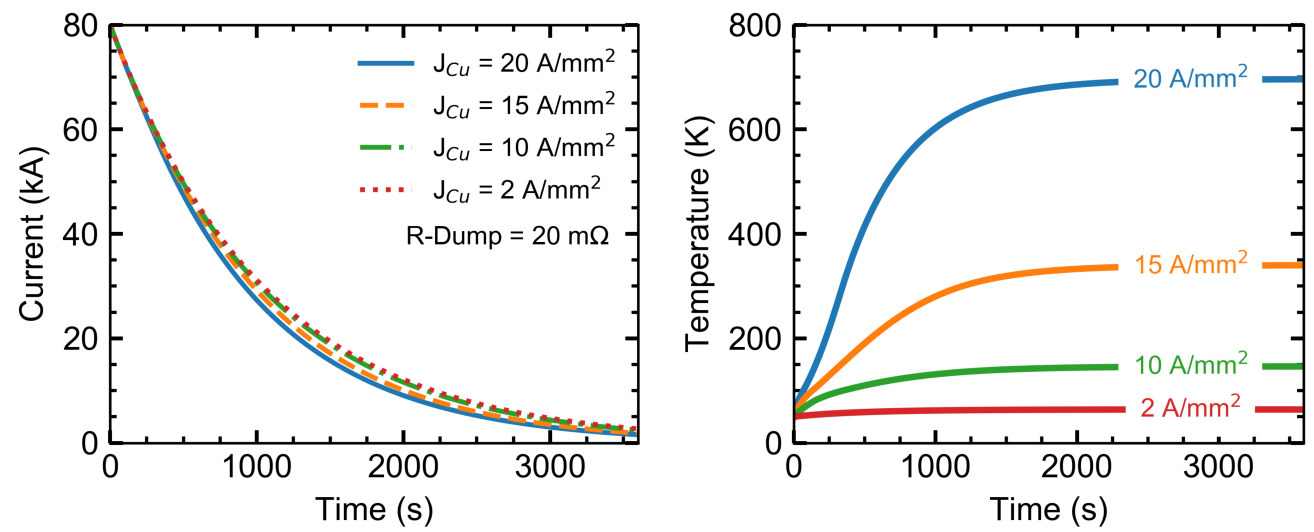

Figure 5.42: Current extraction time and hot spot temperature of the CORC bus bars as function of time using the design for various stabilizer current densities using the operating parameters of the Twin Solenoid detector magnet design. An acceptable hot spot temperature of $150 \mathrm{~K}$ is reached with a stabilizer current density of $10 \mathrm{~A} / \mathrm{mm}^{2}$.

Superconducting bus bars, either based on the ReBCO CORC conductor or other superconducting cables, show advantages regarding several aspects compared to their normal conducting counterparts. However, superconducting bus bars also increase the complexity and costs of the entire system. It is likely that many future large magnet systems will use superconducting (ReBCO CORC based) bus lines when the cost of the superconducting materials drops. At the time of writing, the ReBCO coated conductors are still too valuable to use on large scale. 


\subsection{Conclusion}

Three unique and first in the world demonstrator ReBCO CORC Cable-In-Conduit Conductors were prepared in a common effort of CERN and Advanced Conductor Technologies. All three conductors use the six-around-one cabling concept, implement joint terminals of the latest design, but differentiate in cooling method and jacket material. Characterization tests in the temperature range of 4.2 to $77 \mathrm{~K}$ and a magnetic field range of 5 to $11 \mathrm{~T}$, demonstrated the expected good performance of two out of the three Cable-In-Conduit Conductors. The third conductor showed severe degradation after the in-field tests due to the large electro-magnetic loads on the CORC strands. The two well performing CORC Cable-In-Conduit Conductors show that preparation methods and calculation methods to determine the conductor performance are valid and can be used for future CORC based Cable-In-Conduit Conductors. The degradation observed in the third conductor was traced back to a cabling parameter unique to the strands used in this conductor, which is used as feedback in the production of a new CORC Cable-In-Conduit Conductor to be tested the autumn of 2018 .

Three cooling methods were applied during the tests; liquid bath cooling, forced-flow gas cooling and conduction cooling. All three cooling methods did allow stable operation of the CORC Cable-In-Conduit Conductor and demonstrated their feasibility.

The joint terminals of the three conductors were performing close to their expected performance, but still showed several points for improvement in terms of either resistance or current distribution. The, in total, four terminals of the detector and fusion type CORC cable-in-conduit conductors have resistances of $2.1,2.1,4.3$ and $4.1 \mathrm{n} \Omega(1.9 \mathrm{n} \Omega$ expected) at $5 \mathrm{~K}$ and 4.5, 5.7, 8.0 and $11.1 \mathrm{n} \Omega(6.0 \mathrm{n} \Omega$ expected) at $50 \mathrm{~K}$. The two 'top' terminals have resistances very close to their expected values over the entire temperature range. The two 'bottom' terminals have resistances of several $\mathrm{n} \Omega$ higher than predicted. The higher resistances of the bottom terminals is possibly caused by inhomogeneous current distribution resulting from the degradation of the Cu-jacketed conductor. Also, a difference (as expected for ramp-rate conditions) between the performance of joint terminals making a 'shaking hands' type splice and terminals making a 'praying hands' type splice was observed. The terminals that made the 'shaking hand' type splice are performing better compared to the terminal that made the 'praying hands' type splice both in DC operating and during ramping. The difference in performance is not fully explained yet, though in practice most joints of such conductor in future large magnet system are likely of the 'shaking hand' splice type. The research on joint terminals for the CORC Cable-In-Conduit Conductor is ongoing to further improve joint resistance and current distribution among CORC strands.

The CORC technology has taken a great leap forward with the preparation and testing of three state-of-the-art CORC based Cable-In-Conduit Conductors. More CORC Cable-InConduit Conductors of longer length and with a higher current rating are expected in the near future and possibly to be implement in future large magnet systems either as main conductor or as superconducting bus bar. 


\section{Chapter 6}

\section{Conclusion}

The work reported in this thesis aims at advancing the development of CORC technology by enabling high current density CORC wires and cables for high-field magnets, as well as highcurrent CORC Cable-In-Conduit Conductors for large-scale magnets and their bus lines. One of the key results is the demonstration of a novel type of joint terminal for CORC wires, cables and CICCs that allows for a drastic reduction in joint resistance and for a near-homogeneous current distribution.

\subsection{CORC Wires and Cables for High-Field Magnets}

CORC cables, assembled from standard SuperPower ReBCO tapes of $4 \mathrm{~mm}$ wide with a $50 \mu \mathrm{m}$ Hastelloy substrate, are relatively thick with a typical diameter of more than $5 \mathrm{~mm}$. Hence, they offer a relatively low overall current density due to the large volume fraction of the normal conducting core. This renders them less-suited for compact high-field magnets, in particular for accelerators, where high current density is a key prerequisite. In large-scale magnets for fusion experiments and for particle detectors on the other hand, the conductor's current density is not defining and may be designed significantly lower, in favor of increased electro-magnetic and thermal stability. The lowest achievable diameter of a CORC cable is essentially limited by the minimum bending diameter of the ReBCO tapes. Reducing the Hastelloy thickness from 50 to $30 \mu \mathrm{m}$ allows to lower the minimum bending radius of the tapes and is therefore a necessary development to enable the preparation of CORC wires with a diameter below $4 \mathrm{~mm}$ and an overall current density exceeding $300 \mathrm{~A} / \mathrm{mm}^{2}$ at $20 \mathrm{~T}$ and $4.2 \mathrm{~K}$.

Four such new CORC wires, each with different tape-, layer- and core layout, were developed in cooperation with Advanced Conductor Technologies and characterized at the University of Twente in order to demonstrate their electrical and mechanical performance and their flexibility, as well as to identify which issues can be improved in the conductor or in its production process. After the tests, individual tapes were extracted from the CORC wires and analyzed separately. The following conclusions are drawn: 
- CORC wires with either 2 or $3 \mathrm{~mm}$ wide tapes were tested. Bending the CORC wire assembled from $3 \mathrm{~mm}$ wide tape caused buckling of tapes in some of the bends with a radius close to $30 \mathrm{~mm}$, resulting in severe and irreversible critical current degradation in these sections. CORC wires with $2 \mathrm{~mm}$ wide tape did not show this effect. Therefore, ReBCO tapes of $2 \mathrm{~mm}$ or even less in width have to be used in CORC wires for compact magnets, where flexibility is of the highest importance for ease of winding.

- Two few-turn solenoids were wound from CORC wires. The wires, both assembled from tapes with a $30 \mu \mathrm{m}$ thick substrate, had respective diameters of 3.5 and $4.1 \mathrm{~mm}$. The coils were tested with the Lorentz force pointing either inwards (tensile hoop stress) or outwards (compressive hoop stress). The CORC wire with the inwards force showed a $10 \%$ degradation of the critical current after 14 excitation cycles. However, no degradation was observed with the Lorentz force pointing outwards. Better performance of CORC wires may thus be expected under tension. Presumable, compressive hoop stress on the wire leads to stress concentrations within the tapes that is beyond its limit and thus some level of cracking of the ReBCO layer.

- The minimum bending radius of ReBCO tapes with a substrate thickness of $30 \mu \mathrm{m}$ corresponds, as expected, to a compressive strain limit of $-0.8 \%$ in the ReBCO layer. Major tape degradation is observed beyond this limit. Tapes with even thinner substrates, in the range of 25 to $20 \mu \mathrm{m}$, or with the ReBCO layer centered within the tape's cross-section will not only allow for a further increase in overall current density, but also for a decrease in the minimum diameter of CORC wires, since such development will reduce the tape's minimum bending radius.

- Tape-cabling tension is an important factor in possible later degradation caused by the bending of CORC wires. Excessive tension levels hamper the sliding of tapes, resulting in higher local stress during wire bending. On the other hand, insufficient cabling tension increases the risk of tape buckling, since tapes are more likely to get caught between the tapes of the next layer. The degradation observed in CORC wire nr. 3 mainly occurred in its outer layers, which cannot be correlated with the bending radius of the affected tapes and is therefore likely a result of inadequate tape-cabling tension. This tension level has to be optimized for each specific CORC conductor layout, taking into account its envisioned application.

- Torque applied along the axis of a CORC conductor (i.e. conductor 'twisting') can cause degradation in the ReBCO tapes of every second layer, since some layers are tightened while others are loosened. Such periodic degradation was observed in CORC wire nr. 4. Therefore, it is important to avoid axial torque while bending a CORC conductor.

The latest CORC wire supported a current density of $412 \mathrm{~A} / \mathrm{mm}^{2}$ at $4.2 \mathrm{~K}$ and $10 \mathrm{~T}$, which is similar to the value carried by NbTi Rutherford cables in LHC dipole magnets. It should be noted that this wire comprises ReBCO tapes with quite a low lift-factor of 1.8, where 2.7 was expected, and suffered an additional $18 \%$ degradation due to axial torque and bending. It is therefore not unreasonable to extrapolate that future CORC wires with a higher lift factor, additional Zr-doping and optimized tape-cabling tension may well reach 
substantially higher current densities, in the range of 800 to $1000 \mathrm{~A} / \mathrm{mm}^{2}$ at $4.2 \mathrm{~K}$ and $10 \mathrm{~T}$ and 400 to $500 \mathrm{~A} / \mathrm{mm}^{2}$ at $4.2 \mathrm{~K}$ and $20 \mathrm{~T}$. This will render them excellently suited for high-stability high-field magnets generating fields in the range of 12 to $20 \mathrm{~T}$ and possibly even beyond.

\subsection{CORC Cable-In-Conduit Conductors for Large-Scale Magnets}

CORC multi-strand Cable-In-Conduit Conductors are especially, but not exclusively, of interest to the high energy physics and nuclear fusion communities, for application in future large particle detectors and in tokamak reactors, as well as in the bus bars that feed such magnet systems. Three first-in-the-world CORC CICCs were prepared as proof of concept and to demonstrate their substantial current carrying capacity.

A CORC CICC consists of a cable of multiple twisted CORC strands, enclosed by a highstrength metallic jacket. This work focused on the 'six-around-one' cable layout, in which six CORC strands are helically wound around a central rod or tube. Among the various multi-strand cable layouts that were considered, six-around-one offers an adequate balance between ease of assembly and high current density.

The first CORC six-around-one CICC was assembled and tested at CERN in 2016. This conductor is $1.7 \mathrm{~m}$ long, has an aluminium 5083 jacket and is designed to carry $48 \mathrm{kA}$ at $4.2 \mathrm{~K}$ and $10 \mathrm{~T}$. At $77 \mathrm{~K}$ in self-field, the conductor reached its expected critical current of $12 \mathrm{kA}$ with an n-value of 14 , while at $4.2 \mathrm{~K}$ in a $9 \mathrm{~T}$ background field, it remained fully superconducting up to the test facility's compliance limit of $32 \mathrm{kA}$. These test results convincingly demonstrate that the chosen assembly methods for CORC CICCs are sound and that the ReBCO CORC strands within the cable display a high degree of electrical and thermal stability during its operation.

Next, two CORC CICCs with increased length and -current rating were developed: each is $2.7 \mathrm{~m}$ long and assembled from new high-performance CORC strands to boost the rating to $80 \mathrm{kA}$ at $5 \mathrm{~K}$ and $12 \mathrm{~T}$. These CICCs were specifically designed to meet the operating requirements of detector- and fusion-type magnets. One conductor has a high-stability copper jacket and is conduction cooled, the preferred solution for particle detector magnets. The second conductor has a stainless steel jacket and internal forced-flow gas cooling, as dictated by the stringent demands on mechanical stress- and heat load management in typical fusion magnets.

The tests of these cables demonstrated that stable DC operation of CORC CICCs at a current of $50 \mathrm{kA}$ in magnetic fields over $8 \mathrm{~T}$ is feasible, both with conduction cooling via the conductor's jacket and with internal forced-flow gas cooling. Heat is mainly produced in the joint terminals and by eddy- and coupling current losses during ramping. AC operation is possible as long as the heat generated by AC-losses can be extracted by the cold helium gas. 
The CORC strands in the copper-jacketed CICC suffered significant local degradation under electro-magnetic loading, reducing the critical current to approximately 35\% of its design value. At a temperature of $5 \mathrm{~K}$ and applied field of $10.9 \mathrm{~T}$, this limited the maximum current that could be sustained stably to $50 \mathrm{kA}$. This implied that also the steel-jacketed CICC could not be tested up to its transition current at temperatures below $30 \mathrm{~K}$, since both cables were powered in series. However, the critical current of the steeljacketed CICC could be measured in the temperature range of 40 to $60 \mathrm{~K}$ in external magnetic fields ranging from 5 to $10.9 \mathrm{~T}$ and was found to agree with its design value. The conductor's n-value was measured to be 14 , similar to the first CORC CICC.

Post-mortem inspection of the CORC strands in the degraded copper-jacketed CICC showed strand 'pinching' to be the responsible failure mode. Pinching refers to significant and irreversible deformation of the CORC strands by an electro-magnetic load applied asymmetrically with respect to the strand's axis, which locally pushes the strain in the ReBCO tapes over its maximum allowed limit. This degradation mechanism was traced back to a cabling parameter that is unique to the strands used in the copper-jacketed conductor. Therefore, it is likely to be an unfortunate artifact associated with this specific sample, which can be avoided in follow-up conductors.

In such a follow up, the most significant gains can be expected from the cabling process of the six-around-one cable, from the layout of the CORC strands and from the strand production process. Twisting of the CORC strands is now done manually and is likely to benefit from mechanical aids that allow to apply a more uniform twist to the cable bundle. This will reduce the variation in the cable's outer diameter along its length and thus allow for a better fit and support of the strands inside the jacket. The specific layout of the CORC strands in the copper-jacketed CICC caused their degradation. Using a thicker, more sturdy strand core will reduce the pinching effect that occurred under the high electro-magnetic loads. Further research into the detailed influence of the CORC strand layout on its mechanical response to complex electro-magnetic loads will be instrumental in boosting CICC performance.

In this present pioneering work, CORC Cable-In-Conduit Conductors were demonstrated to sustain current levels of at least $32 \mathrm{kA}$ at $4.2 \mathrm{~K}$ and $9 \mathrm{~T}$ and of $50 \mathrm{kA}$ at $5 \mathrm{~K}$ and $12 \mathrm{~T}$, which correspond to overall current densities of 36 and $56 \mathrm{~A} / \mathrm{mm}^{2}$, respectively. Reasonable extrapolation leads to expected currents up to $80 \mathrm{kA}$ at $5 \mathrm{~K}$ and $12 \mathrm{~T}\left(89 \mathrm{~A} / \mathrm{mm}^{2}\right)$ to be feasible. It should be noted not only that these values surpass the current density in the ATLAS barrel toroid magnet $\left(30 \mathrm{~A} / \mathrm{mm}^{2}\right.$ at $4.5 \mathrm{~K}$ and $\left.3 \mathrm{~T}\right)$ and in the ITER CS and TF conductors $\left(19 \mathrm{~A} / \mathrm{mm}^{2}\right.$ and $45 \mathrm{~A} / \mathrm{mm}^{2}$ at $4.5 \mathrm{~K}$ and $12-13 \mathrm{~T}$, respectively), but also that ReBCO CORC CICC technology provides an order-of-magnitude increase in electromagnetic and thermal stability.

\subsection{Novel Joint Terminals for CORC Conductors}

Low-resistance joint terminals for CORC conductors are essential for their application, even to the point that the joints may be argued to determine to a large extent the conductor's performance. A low and well-distributed joint resistance is important for two distinct 
reasons: it limits the ohmic energy dissipation in the joint, which under normal operation constitutes the main heat load on the conductor; and an identical contact resistance to each $R e B C O$ tape drives a homogeneous DC current distribution throughout the CORC conductor and hence results in maximum electro-magnetic and thermal stability.

A novel joint terminal design is introduced that leads to a significant reduction in joint resistance and to a much more homogeneous current distribution within the CORC conductors. In this new design, the ReBCO layers in the conductor ends are staged to a staircase-like pattern by cutting away part of each layer of tapes in the CORC joint section. This allows to inject the current directly into each layer, without having to pass the resistive substrate and buffer layers of the tapes of the outermost layers.

Numerical predictions show a major improvement in the current distribution among the ReBCO layers when comparing old and new joint designs. In theory, a perfectly uniform current distribution can be achieved by optimizing the length of each stage. For example, in a CORC conductor with $12 \mathrm{ReBCO}$ layers the new joint design causes a reduction of the overall contact resistance by $80 \%$ by eliminating the resistive Hastelloy and buffer layers from the current path. It also makes this resistance value independent of the current level, which was not the case in earlier joint designs.

A practical staging protocol is developed to prepare the ends of CORC conductors for insertion in metal joint terminals. A 'bandage' of tin-lead solder is applied around each stage, which makes sure that none of the tapes 'spring out' radially before insertion. This solder bandage melts when the joint terminals are later filled with solder, at which point the tapes do spring out slightly and solder can flow more easily in-between them. Currently, tin-lead based solders are successfully used at CERN for CORC joint preparation. The use of other solder materials, such as indium, as well as tapes with thinner substrates are expected to reduce the resistance of the CORC joint terminals by a further 20 to $80 \%$, depending on the detailed joint configuration. Indium is also mechanically softer than any of the tin-lead solders and will therefore reduce thermal stresses in the ReBCO tapes caused by the differential thermal contraction of materials.

Indicative engineering values for the joint resistance at $4.2 \mathrm{~K}$ as function of the joint length $L$ were numerically estimated with the nodal-network model for typical CORC wires, for cables and for cable-in-conduit conductors:

$$
\begin{array}{rlrl}
R_{\text {Wire }}(L) & =1.8 \cdot 10^{-9}[\Omega \mathrm{m}] / L[\mathrm{~m}], & \\
R_{\text {Cable }}(L) & =2.8 \cdot 10^{-9}[\Omega \mathrm{m}] / L[\mathrm{~m}], & \\
R_{\text {CICC }}(L) & =0.6 \cdot 10^{-9}[\Omega \mathrm{m}] / L[\mathrm{~m}], & & \text { (single side injection) } \\
R_{\text {CICC }}(L) & =0.4 \cdot 10^{-9}[\Omega \mathrm{m}] / L[\mathrm{~m}] . & & \text { (double sided injection) }
\end{array}
$$

Note that these numbers merely provide a rough first estimate of the expected joint resistance. The actual resistance of a given CORC joint will depend on its layout and on the layout of the cable, which will result in some variation around these cited values.

Experiments demonstrated that low and current-independent values of the joint resistance can indeed be achieved which are close to the model predictions. However, these tests also revealed that the preparation protocols may still be improved further, since some 
of the joint terminals showed a higher than expected resistance value, likely due to gas pockets and other voids in the terminals.

The new joint terminal design can be scaled up for use with the CORC Cable-In-Conduit Conductor. In the course of this work, seven joint terminals for CORC CICCs were prepared: one trial prototype and six fully functional terminals for the three realized and tested CORC CICCs.

A uniform current distribution requires each of the CORC strands in a CICC to have a similar contact resistance, especially in short-sample cables with a low self-inductance. The largest current imbalance can occur when current is injected only in one side of a joint terminal, since the contact resistance associated with the strands located closest to this injection plane is lower than for those located further away. However, the current distribution in the six-around-one CICCs can be improved to near-uniform by controlling the strand positions inside both terminals simultaneously, in such fashion that strands which have a relatively high contact resistance in one terminal experience a lower one in the other, evening-out the total contact resistance of each strand.

Adequate performance of the joint terminals of the CORC CICCs was extensively verified. Their resistance as function of temperature behaves as expected, but is commonly a few $\mathrm{n} \Omega$ higher than the model predictions, likely due to a combination of the magnetoresistance of the copper and the presence of residual gas pockets or voids in the solder filling. The current was found to be distributed evenly among the CORC strands in each of the CICC samples, which is a convincing validation of the new joint technology. Also, joint terminals in a shaking-hands type of splice had a lower resistance and lead to a more uniform current distribution than those in a praying-hands type of splice. The exact nature of this phenomenon has to be investigated in more detail. 


\section{Bibliography}

[1] M. N. Wilson, "NbTi superconductors with low AC loss: A review", Cryogenics, vol. 48, no. 7-8, pp. 381-395, 2008.

[2] A. Zlobin, G. Ambrosio, N. Andreev, E. Barzi, R. Bossert, R. Carcagno, G. Chlachidze, J. DiMarco, S. Feher, V. Kashikhin, et al., " $\mathrm{Nb}_{3} \mathrm{Sn}$ accelerator magnet technology R\&D at Fermilab", Proceedings of the Particle Accelerator Conference, pp. 482-484, 2007.

[3] S. Caspi and P. Ferracin, "Limits of $\mathrm{Nb}_{3} \mathrm{Sn}$ accelerator magnets", Proceedings of the Particle Accelerator Conference, pp. 107-111, 2005.

[4] R. Perin, "The superconducting magnet system for the LHC", IEEE Transactions on Magnetics, vol. 27, no. 2, pp. 1735-1742, 1991.

[5] P. Pugnat and A. Siemko, "Review of quench performance of LHC main superconducting magnets", IEEE Transactions on Applied Superconductivity, vol. 17, no. 2, pp. 1091-1096, 2007.

[6] H. H. J. ten Kate, "The superconducting magnet system for the ATLAS detector at CERN", IEEE Transactions on Applied Superconductivity, vol. 10, no. 1, pp. 347-352, 2000.

[7] H. H. J. ten Kate, "The ATLAS superconducting magnet system at the Large Hadron Collider", Physica C: Superconductivity, vol. 468, no. 15-20, pp. 2137-2142, 2008.

[8] N. Mitchell, D. Bessette, R. Gallix, C. Jong, J. Knaster, P. Libeyre, C. Sborchia, and F. Simon, "The ITER magnet system", IEEE Transactions on Applied Superconductivity, vol. 18, no. 2, pp. 435-440, 2008.

[9] A. Ballarino, "Development of superconducting links for the Large Hadron Collider machine", Superconductor Science and Technology, vol. 27, no. 4, Art. ID 044024, 2014.

[10] M.-K. Wu, J. R. Ashburn, C. Torng, P. H. Hor, R. L. Meng, L. Gao, Z. J. Huang, $\mathrm{Y}$. Wang, and a. Chu, "Superconductivity at $93 \mathrm{~K}$ in a new mixed-phase Y-Ba-Cu-O compound system at ambient pressure", Physical Review Letters, vol. 58, no. 9, Art. ID 908, 1987. 
[11] K. H. Sandhage, G. N. Riley, and W. L. Carter, "Critical issues in the OPIT processing of high-J $\mathrm{J}_{c}$ BSCCO superconductors", Journal of the Minerals, Metals \& Materials Society, vol. 43, no. 3, pp. 21-25, 1991.

[12] A. Malozemoff, S. Fleshler, M. Rupich, C. Thieme, X. Li, W. Zhang, A. Otto, J. Maguire, D. Folts, J. Yuan, et al., "Progress in high temperature superconductor coated conductors and their applications", Superconductor Science and Technology, vol. 21, no. 3, Art. ID 034005, 2008.

[13] V. Selvamanickam, Y. Chen, X. Xiong, Y. Xie, X. Zhang, A. Rar, M. Martchevskii, R. Schmidt, K. Lenseth, and J. Herrin, "Progress in second-generation HTS wire development and manufacturing", Physica C: Superconductivity, vol. 468, no. 15-20, pp. 1504-1509, 2008.

[14] T. Sekitani, N. Miura, S. Ikeda, Y. Matsuda, and Y. Shiohara, "Upper critical field for optimally-doped $\mathrm{YBa}_{2} \mathrm{Cu}_{3} \mathrm{O}_{7-\delta}$ ", Physica B: Condensed Matter, vol. 346, pp. 319324, 2004.

[15] W. D. Markiewicz, D. C. Larbalestier, H. W. Weijers, A. J. Voran, K. W. Pickard, W. R. Sheppard, J. Jaroszynski, A. Xu, R. P. Walsh, J. Lu, et al., "Design of a superconducting 32 T magnet with REBCO high field coils", IEEE Transactions on Applied Superconductivity, vol. 22, no. 3, Art. ID 4300704, 2012.

[16] Y. Iwasa, "HTS magnets: stability; protection; cryogenics; economics; current stability/protection activities at FBML", Cryogenics, vol. 43, no. 3-5, pp. 303-316, 2003.

[17] J. Maguire, F. Schmidt, S. Bratt, T. Welsh, J. Yuan, A. Allais, and F. Hamber, "Development and demonstration of a HTS power cable to operate in the long island power authority transmission grid", IEEE Transactions on Applied Superconductivity, vol. 17, no. 2, pp. 2034-2037, 2007.

[18] M. Stemmle, F. Merschel, M. Noe, and A. Hobl, "Ampacity project-worldwide first superconducting cable and fault current limiter installation in a German city center", presented at the 22nd International Conference and Exhibition on Electricity Distribution, 2013.

[19] W. Goldacker, F. Grilli, E. Pardo, A. Kario, S. I. Schlachter, and M. Vojenčiak, "Roebel cables from REBCO coated conductors: A one-century-old concept for the superconductivity of the future", Superconductor Science and Technology, vol. 27, no. 9, Art. ID 093001, 2014.

[20] R. A. Badcock, N. J. Long, M. Mulholland, S. Hellmann, A. Wright, and K. A. Hamilton, "Progress in the manufacture of long length YBCO Roebel cables", IEEE Transactions on Applied Superconductivity, vol. 19, no. 3, pp. 3244-3247, 2009.

[21] M. Takayasu, L. Chiesa, L. Bromberg, and J. V. Minervini, "HTS twisted stackedtape cable conductor", Superconductor Science and Technology, vol. 25, no. 1, Art. ID 014011, 2011. 
[22] D. C. van der Laan, " $\mathrm{YBa}_{2} \mathrm{Cu}_{3} \mathrm{O}_{7}-\delta$ coated conductor cabling for low ac-loss and high-field magnet applications", Superconductor Science and Technology, vol. 22, no. 6, Art. ID 065013, 2009.

[23] D. C. van der Laan, X. Lu, and L. F. Goodrich, "Compact $\mathrm{GdBa}_{2} \mathrm{Cu}_{3} \mathrm{O}_{7-\delta}$ coated conductor cables for electric power transmission and magnet applications", Superconductor Science and Technology, vol. 24, no. 4, Art. ID 042001, 2011.

[24] L. Roebel, "Electrical conductor.", June 22 1915. US Patent 1,144,252.

[25] M. N. Wilson, "Superconductivity and accelerators: the good companions", IEEE Transactions on Applied Superconductivity, vol. 9, no. 2, pp. 111-121, 1999.

[26] W. Goldacker, R. Nast, G. Kotzyba, S. Schlachter, A. Frank, B. Ringsdorf, C. Schmidt, and P. Komarek, "High current DyBCO-ROEBEL assembled coated conductor (RACC)", Journal of Physics: Conference Series, vol. 43, no. 1, pp. 901-904, 2006.

[27] G. A. Kirby, J. van Nugteren, H. Bajas, V. Benda, A. Ballarino, M. Bajko, L. Bottura, K. Broekens, M. Canale, A. Chiuchiolo, et al., "First cold powering test of REBCO roebel wound coil for the EuCARD2 future magnet development project", IEEE Transactions on Applied Superconductivity, vol. 27, no. 4, pp. 1-7, 2017.

[28] M. Takayasu, L. Chiesa, N. C. Allen, and J. V. Minervini, "Present status and recent developments of the twisted stacked-tape cable conductor", IEEE Transactions on Applied Superconductivity, vol. 26, no. 2, pp. 25-34, 2016.

[29] J. D. Weiss, T. Mulder, H. H. J. ten Kate, and D. C. van der Laan, "Introduction of CORC ${ }^{\circledR}$ wires: highly flexible, round high-temperature superconducting wires for magnet and power transmission applications", Superconductor Science and Technology, vol. 30, no. 1, Art. ID 014002, 2016.

[30] T. Mulder, A. Dudarev, M. Mentink, H. Silva, D. van der Laan, M. Dhallé, and H. H. J. ten Kate, "Design and manufacturing of a $45 \mathrm{kA}$ at $10 \mathrm{~T}$ ReBCO-CORC cable-in-conduit conductor for large-scale magnets", IEEE Transactions on Applied Superconductivity, vol. 26, no. 4, pp. 1-5, 2016.

[31] A. Dudarev, M. Doser, D. Perini, and H. H. J. ten Kate, "Design of a Superconducting Magnet System for the AEGIS Experiment at CERN", IEEE Transactions on Applied Superconductivity, vol. 21, no. 3, pp. 1721-1724, 2011.

[32] L. Rossi, "The LHC main dipoles and quadrupoles toward series production", IEEE Transactions on Applied Superconductivity, vol. 13, no. 2, pp. 1221-1228, 2003.

[33] P. Ferracin, M. Devaux, M. Durante, P. Fazilleau, P. Fessia, P. Manil, A. Milanese, J. M. Garcia, L. Oberli, J. Perez, et al., "Development of the EuCARD $\mathrm{Nb}_{3} \mathrm{Sn}$ Dipole Magnet FRESCA2", IEEE Transactions on Applied Superconductivity, vol. 23, no. 3, pp. 4002005-4002005, 2013.

[34] J. Fleiter, A. Ballarino, L. Bottura, W. Goldacker, and A. Kario, "Characterization of Roebel cables for potential use in high-field magnets", IEEE Transactions on Applied Superconductivity, vol. 25, no. 3, pp. 1-4, 2015. 
[35] T. Mulder, D. van der Laan, J. Weiss, A. Dudarev, M. Dhallé, and H. H. J. ten Kate, "Design and Preparation of Two ReBCO-CORC ${ }^{\circledR}$ Cable-In-Conduit Conductors for Fusion and Detector Magnets", IOP Conference Series: Materials Science and Engineering, vol. 279, no. 1, Art. ID 012033, 2017.

[36] J. Sapper et al., "Superconducting coil development for the Wendelstein 7-X stellarator", Fusion Engineering and Design, vol. 20, pp. 23-32, 1993.

[37] A. Devred, I. Backbier, D. Bessette, G. Bevillard, M. Gardner, M. Jewell, N. Mitchell, I. Pong, and A. Vostner, "Status of ITER conductor development and production", IEEE Transactions on Applied Superconductivity, vol. 22, no. 3, pp. 48049094804909, 2012.

[38] G. Celentano, G. De Marzi, F. Fabbri, L. Muzzi, G. Tomassetti, A. Anemona, S. Chiarelli, M. Seri, A. Bragagni, and A. della Corte, "Design of an industrially feasible twisted-stack HTS cable-in-conduit conductor for fusion application", IEEE Transactions on Applied Superconductivity, vol. 24, no. 3, pp. 1-5, 2014.

[39] D. Uglietti, N. Bykovsky, K. Sedlak, B. Stepanov, R. Wesche, and P. Bruzzone, "Test of $60 \mathrm{kA}$ coated conductor cable prototypes for fusion magnets", Superconductor Science and Technology, vol. 28, no. 12, Art. ID 124005, 2015.

[40] D. van der Laan, "Conductor on Round Core (CORC) cable development for accelerator magnets", presented at WAMHTS-2014, 2014.

[41] D. M. Feldmann, T. G. Holesinger, R. Feenstra, and D. C. Larbalestier, "A review of the influence of grain boundary geometry on the electromagnetic properties of polycrystalline $\mathrm{YBa}_{2} \mathrm{Cu}_{3} \mathrm{O}_{7-x}$ films", Journal of the American Ceramic Society, vol. 91, no. 6, pp. 1869-1882, 2008.

[42] C. Wang, K. Do, M. Beasley, T. Geballe, and R. Hammond, "Deposition of in-plane textured $\mathrm{MgO}$ on amorphous $\mathrm{Si}_{3} \mathrm{~N}_{4}$ substrates by ion-beam-assisted deposition and comparisons with ion-beam-assisted deposited yttria-stabilized-zirconia", Applied Physics Letters, vol. 71, no. 20, pp. 2955-2957, 1997.

[43] A. Goyal, M. P. Paranthaman, and U. Schoop, "The RABiTS approach: Using rollingassisted biaxially textured substrates for high-performance YBCO superconductors", MRS bulletin, vol. 29, no. 8, pp. 552-561, 2004.

[44] A. Gupta, R. Jagannathan, E. I. Cooper, E. Giess, J. Landman, and B. Hussey, "Superconducting oxide films with high transition temperature prepared from metal trifluoroacetate precursors", Applied Physics Letters, vol. 52, no. 24, pp. 2077-2079, 1988.

[45] P. Chou, Q. Zhong, Q. Li, K. Abazajian, A. Ignatiev, C. Wang, E. Deal, and J. Chen, "Optimization of $\mathrm{J}_{c}$ of YBCO thin films prepared by photo-assisted MOCVD through statistical robust design", Physica C: Superconductivity, vol. 254, no. 1-2, pp. 93112, 1995.

[46] K. Hasegawa, K. Fujino, H. Mukai, M. Konishi, K. Hayashi, K. Sato, S. Honjo, Y. Sato, H. Ishii, and Y. Iwata, "Biaxially aligned YBCO film tapes fabricated by all pulsed 
laser deposition", IEEE Transactions on Applied Superconductivity, vol. 4, no. 10, pp. 487-493, 1996.

[47] V. Matias, E. J. Rowley, Y. Coulter, B. Maiorov, T. Holesinger, C. Yung, V. Glyantsev, and B. Moeckly, "YBCO films grown by reactive co-evaporation on simplified IBAD$\mathrm{MgO}$ coated conductor templates", Superconductor Science and Technology, vol. 23, no. 1, Art. ID 014018, 2009.

[48] V. Matias, "Perspectives on IBAD Coated Conductors: Cost and Future", CCA2016, 2016.

[49] "Superpower Inc. website", http://www.superpower-inc.com/content/2g-hts-wire. Accessed: 2018-04-16.

[50] J. Kellers, "Development of a Superconductive Wind Power Generator within the EcoSwing Project", presented at MT-25, 2017.

[51] K. Tsuchiya, A. Kikuchi, A. Terashima, K. Norimoto, M. Uchida, M. Tawada, M. Masuzawa, N. Ohuchi, X. Wang, T. Takao, et al., "Critical current measurement of commercial REBCO conductors at 4.2 K", Cryogenics, vol. 85, pp. 1-7, 2017.

[52] K. Tsuchiya, A. Kikuchi, A. Terashima, K. Suzuki, K. Norimoto, M. Tawada, M. Masuzawa, N. Ohuchi, X. Wang, Y. Iijima, et al., "Critical current characterization of commercial REBCO coated conductors at 4.2 and 77 K", IEEE Transactions on Applied Superconductivity, vol. 27, no. 4, pp. 1-5, 2017.

[53] V. Braccini, A. Xu, J. Jaroszynski, Y. Xin, D. Larbalestier, Y. Chen, G. Carota, J. Dackow, I. Kesgin, Y. Yao, et al., "Properties of recent IBAD-MOCVD coated conductors relevant to their high field, low temperature magnet use", Superconductor Science and Technology, vol. 24, no. 3, Art. ID 035001, 2010.

[54] L. Stan, T. Holesinger, B. Maiorov, Y. Chen, D. Feldmann, I. Usov, R. DePaula, V. Selvamanickam, L. Civale, S. Foltyn, et al., "Structural and superconducting properties of $(\mathrm{Y}, \mathrm{Gd}) \mathrm{Ba}_{2} \mathrm{Cu}_{3} \mathrm{O}_{7-\delta}$ grown by MOCVD on samarium zirconate buffered IBADMgO", Superconductor Science and Technology, vol. 21, no. 10, Art. ID 105023, 2008.

[55] B. Maiorov, S. Baily, H. Zhou, O. Ugurlu, J. Kennison, P. Dowden, T. Holesinger, S. Foltyn, and L. Civale, "Synergetic combination of different types of defect to optimize pinning landscape using BaZrO3-doped $\mathrm{YBa}_{2} \mathrm{Cu}_{3} \mathrm{O}_{7}$ ", Nature materials, vol. 8, no. 5, pp. 398-404, 2009.

[56] D. Hazelton, "Progress of Wire Development and Process Improvement on 2G HTS at SuperPower", presented at EUCAS-2017, 2017.

[57] D. Hilton, A. Gavrilin, and U. Trociewitz, "Practical fit functions for transport critical current versus field magnitude and angle data from (RE)BCO coated conductors at fixed low temperatures and in high magnetic fields", Superconductor Science and Technology, vol. 28, no. 7, Art. ID 074002, 2015.

[58] A. Sundaram, Y. Zhang, A. Knoll, D. Abraimov, P. Brownsey, M. Kasahara, G. Carota, R. Nakasaki, J. Cameron, G. Schwab, et al., "2G HTS wires made on $30 \mu \mathrm{m}$ thick 
Hastelloy substrate", Superconductor Science and Technology, vol. 29, no. 10, Art. ID 104007, 2016.

[59] M. Vojenčiak, A. Kario, B. Ringsdorf, R. Nast, D. van der Laan, J. Scheiter, A. Jung, B. Runtsch, F. Gömöry, and W. Goldacker, "Magnetization AC loss reduction in HTS CORC ${ }^{\circledR}$ cables made of striated coated conductors", Superconductor Science and Technology, vol. 28, no. 10, Art. ID 104006, 2015.

[60] D. C. van der Laan, D. Abraimov, A. Polyanskii, D. C. Larbalestier, J. Douglas, R. Semerad, and M. Bauer, "Anisotropic in-plane reversible strain effect in $\mathrm{Y}_{0.5} \mathrm{Gd}_{0.5} \mathrm{Ba}_{2} \mathrm{Cu}_{3} \mathrm{O}_{7-\delta}$ coated conductors", Superconductor Science and Technology, vol. 24, no. 11, Art. ID 115010, 2011.

[61] H.-S. Shin, K.-H. Kim, J. R. C. Dizon, T.-Y. Kim, R.-K. Ko, and S.-S. Oh, "The strain effect on critical current in YBCO coated conductors with different stabilizing layers", Superconductor Science and Technology, vol. 18, no. 12, Art. ID S364, 2005.

[62] N. Cheggour, J. Ekin, Y.-Y. Xie, V. Selvamanickam, C. Thieme, and D. Verebelyi, "Enhancement of the irreversible axial-strain limit of Y-Ba-Cu-O-coated conductors with the addition of a Cu layer", Applied Physics Letters, vol. 87, no. 21, Art. ID 212505, 2005.

[63] M. Sugano, K. Shikimachi, N. Hirano, and S. Nagaya, "The reversible strain effect on critical current over a wide range of temperatures and magnetic fields for YBCO coated conductors", Superconductor Science and Technology, vol. 23, no. 8, Art. ID 085013, 2010.

[64] D. C. van der Laan, J. Ekin, J. Douglas, C. Clickner, T. C. Stauffer, and L. Goodrich, "Effect of strain, magnetic field and field angle on the critical current density of $\mathrm{YBa}_{2} \mathrm{Cu}_{3} \mathrm{O}_{7-\delta}$ coated conductors", Superconductor Science and Technology, vol. 23, no. 7, Art. ID 072001, 2010.

[65] S. Otten, A. Kario, A. Kling, and W. Goldacker, "Bending properties of different REBCO coated conductor tapes and Roebel cables at T=77 K", Superconductor Science and Technology, vol. 29, no. 12, Art. ID 125003, 2016.

[66] T. Takao, S. Koizuka, K. Oi, A. Ishiyama, J. Kato, T. Machi, K. Nakao, and Y. Shiohara, "Characteristics of compressive strain and superconducting property in YBCO coated conductor", IEEE Transactions on Applied Superconductivity, vol. 17, no. 2, pp. 3517-3519, 2007.

[67] D. C. van der Laan, J. Ekin, C. C. Clickner, and T. C. Stauffer, "Delamination strength of YBCO coated conductors under transverse tensile stress", Superconductor Science and Technology, vol. 20, no. 8, Art. ID 765, 2007.

[68] A. Kario, "Progress and plan for Roebel", presented at EUCARD-2 WP10, 2016.

[69] D. C. van der Laan, X. Lu, and L. F. Goodrich, "Compact $\mathrm{GdBa}_{2} \mathrm{Cu}_{3} \mathrm{O}_{7-\delta}$ coated conductor cables for electric power transmission and magnet applications", Superconductor Science and Technology, vol. 24, no. 4, Art. ID 042001, 2011. 
[70] T. Mulder, J. Weiss, D. van der Laan, M. Dhalle, and H. H. J. ten Kate, "Development of ReBCO-CORC Wires with Current Densities of 400 to $600 \mathrm{~A} / \mathrm{mm}^{2}$ at $10 \mathrm{~T}$ and 4.2 K", IEEE Transactions on Applied Superconductivity, 2017.

[71] C. Barth, D. van der Laan, N. Bagrets, C. M. Bayer, K.-P. Weiss, and C. Lange, "Temperature-and field-dependent characterization of a conductor on round core cable", Superconductor Science and Technology, vol. 28, no. 6, Art. ID 065007, 2015.

[72] G. Willering, D. van der Laan, H. Weijers, P. Noyes, G. Miller, and Y. Viouchkov, "Effect of variations in terminal contact resistances on the current distribution in high-temperature superconducting cables", Superconductor Science and Technology, vol. 28, no. 3, Art. ID 035001, 2015.

[73] D. van der Laan, F. Douglas, X. Lu, A. de Jager, L. Bromberg, P. Michael, J. Minervini, U. Trociewitz, P. Noyes, G. Miller, et al., "Development of HTS conductor on round core (CORC) cables for fusion applications at advanced conductor technologies", presented at HTS4Fusion, 2014.

[74] D. van der Laan, "Personal Correspondence", 2014.

[75] K. Yagotintsev, W. A. Wessel, A. Vostner, A. Devred, D. Bessette, Y. Nabara, T. Boutboul, V. Tronza, S. Park, W. Yu, et al., "Status of AC loss verification tests on ITER conductors with transverse load cycling", in presented at ICEC/ICMC 2014, 2014.

[76] J. Fleiter and A. Ballarino, "In-Field Electrical Resistance at $4.2 \mathrm{~K}$ of REBCO Splices", IEEE Transactions on Applied Superconductivity, vol. 27, no. 4, pp. 1-5, 2017.

[77] C. Baldan, U. Oliveira, A. Bernardes, V. Oliveira, C. Shigue, and E. Ruppert, "Electrical and superconducting properties in lap joints for YBCO tapes", Journal of superconductivity and novel magnetism, vol. 26, no. 5, pp. 2089-2092, 2013.

[78] J. Zhu, X. Bao, L. Guo, Z. Xia, M. Qiu, and W. Yuan, "Optimal design of current sharing in transmission conductors of a $110 \mathrm{kV} / 3 \mathrm{kA}$ cold dielectric superconducting cable consisted of YBCO tapes", IEEE Transactions on Applied Superconductivity, vol. 23, no. 3, pp. 5402505-5402505, 2013.

[79] S. Mukoyama, K. Miyoshi, H. Tsubouti, T. Yoshida, M. Mimura, N. Uno, M. Ikeda, S. Honjo, N. Hobara, and Y. Iwata, "Uniform current distribution conductor of HTS power cable with variable tape-winding pitches", Advances in Superconductivity XI, pp. 1373-1376, 1999.

[80] S. Mukoyama, K. Miyoshi, H. Tsubouti, M. Mimitra, N. Uno, N. Ichtyanagi, Y. Tanaka, M. Ikeda, H. Ishii, S. Honjo, et al., "50-m long HTS conductor for power cable", IEEE Transactions on Applied Superconductivity, vol. 7, no. 2, pp. 1069-1072, 1997.

[81] J. Ekin, Experimental techniques for low-temperature measurements: cryostat design, material properties and superconductor critical-current testing. Book, Oxford university press, 2006.

[82] A. Clark, G. Childs, and G. Wallace, "Electrical resistivity of some engineering alloys at low temperatures", Cryogenics, vol. 10, no. 4, pp. 295-305, 1970. 
[83] Y. Iwasa, Case studies in superconducting magnets: design and operational issues. Book, Springer Science \& Business Media, 2009.

[84] R. Fast, W. Craddock, M. Kobayashi, and M. Mruzek, "Electrical and mechanical properties of lead/tin solders and splices for superconducting cables", Cryogenics, vol. 28, no. 1, pp. 7-9, 1988.

[85] M. Polak, P. Barnes, and G. Levin, "YBCO/Ag boundary resistivity in YBCO tapes with metallic substrates", Superconductor Science and Technology, vol. 19, no. 8, Art. ID 817, 2006.

[86] S. Yazaki, A. Karasawa, T. Kotoyori, A. Ishiyama, and N. Miyahara, "Critical current degradation in high-temperature superconducting tapes caused by temperature rise", IEEE Transactions on Applied Superconductivity, vol. 23, no. 3, pp. 46023044602304, 2013.

[87] D. Uglietti, "Heating tolerance of critical current in industrial coated conductors", presented at EUCAS-2017, 2017.

[88] C. Senatore, C. Barth, M. Bonura, M. Kulich, and G. Mondonico, "Field and temperature scaling of the critical current density in commercial REBCO coated conductors", Superconductor Science and Technology, vol. 29, no. 1, Art. ID 014002, 2015.

[89] J. Liu, S. Song, Q. Wang, and Q. Zhang, "Critical current analysis of an YBCO insert for ultrahigh-field all-superconducting magnet", IEEE Transactions on Applied Superconductivity, vol. 26, no. 3, pp. 1-6, 2016.

[90] A. Verweij, J. Genest, A. Knezovic, D. Leroy, J.-P. Marzolf, and L. Oberli, "1.9 K test facility for the reception of the superconducting cables for the LHC", IEEE Transactions on Applied Superconductivity, vol. 9, no. 2, pp. 153-156, 1999.

[91] J. Fleiter, A. Ballarino, L. Bottura, and P. Tixador, "Electrical characterization of REBCO Roebel cables", Superconductor Science and Technology, vol. 26, no. 6, Art. ID 065014, 2013.

[92] J. Fleiter, "Personal Correspondence", 2014.

[93] V. Selvamanickam, "Recent developments in high performance REBCO for high magnetic field applications", LTSW, Jacksonville, USA, 2018.

[94] S. Kar, W. Luo, A. B. Yahia, X.-F. Li, G. Majkic, and V. Selvamanickam, “Je (4.2 K, 15 T) beyond $450 \mathrm{~A} / \mathrm{mm}^{2}$ at $15 \mathrm{~mm}$ bend radius with REBCO Symmetric Tape Round (STAR) wire: A prospective candidate for future accelerator magnet applications", Superconductor Science and Technology, 2018.

[95] H. Weijers et al., "The NHMFL $32 \mathrm{~T}$ superconducting magnet", presented at EUCAS2017, 2017.

[96] S. Yoon, J. Kim, K. Cheon, H. Lee, S. Hahn, and S.-H. Moon, "26 T 35 mm all$\mathrm{GdBa}_{2} \mathrm{Cu}_{3} \mathrm{O}_{7-\delta}$ multi-width no-insulation superconducting magnet", Superconductor Science and Technology, vol. 29, no. 4, Art. ID 04LT04, 2016. 
[97] J.-B. Song, S. Hahn, T. Lécrevisse, J. Voccio, J. Bascuñán, and Y. Iwasa, "Overcurrent quench test and self-protecting behavior of a $7 \mathrm{~T} / 78 \mathrm{~mm}$ multi-width no-insulation REBCO magnet at 4.2 K", Superconductor Science and Technology, vol. 28, no. 11, Art. ID 114001, 2015.

[98] J. van Nugteren et al., "HTS Accelerator Magnet Dipole Assembly and Cold Test by CERN for EuCARD2", presented at MT-25, 2017.

[99] X. Wang et al., "Development and test of REBCO canted $\cos \theta$ dipole coils with CORC wires", presented at MT-25, 2017.

[100] J. J. Himbele, A. Badel, and P. Tixador, "Partially insulated twisted stacked cable for HTS insert of a particle accelerator", IEEE Transactions on Applied Superconductivity, vol. 27, no. 4, pp. 1-5, 2017.

[101] J. van Nugteren, G. Kirby, G. de Rijk, L. Rossi, H. H. J. ten Kate, and M. M. Dhalle, "Study of a 5 T research dipole insert-magnet using an anisotropic ReBCO Roebel cable", IEEE Transactions on Applied Superconductivity, vol. 25, no. 3, pp. 1-5, 2015.

[102] S. Rindt et al., "Proof of Concept for ReBCO-CORC Based Magnet Applications", Master Thesis, CERN/TU Eindhoven, 2018.

[103] T. Takematsu, R. Hu, T. Takao, Y. Yanagisawa, H. Nakagome, D. Uglietti, T. Kiyoshi, M. Takahashi, and H. Maeda, "Degradation of the performance of a YBCO-coated conductor double pancake coil due to epoxy impregnation", Physica C: Superconductivity and its Applications, vol. 470, no. 17-18, pp. 674-677, 2010.

[104] M. Morpurgo, "A large superconducting dipole cooled by forced circulation of two phase helium", Cryogenics, vol. 19, no. 7, pp. 411-414, 1979.

[105] N. Abgrall, O. Andreeva, A. Aduszkiewicz, Y. Ali, T. Anticic, N. Antoniou, B. Baatar, F. Bay, A. Blondel, J. Blumer, et al., "NA61/SHINE facility at the CERN SPS: beams and detector system", Journal of Instrumentation, vol. 9, no. 06, Art. ID P06005, 2014.

[106] R. Zanino, M. Bagnasco, D. Ciazynski, B. Lacroix, E. van Lanen, S. Nicollet, A. Nijhuis, L. S. Richard, C. Sborchia, A. Torre, et al., "EU contribution to the test and analysis of the ITER poloidal field conductor insert and the central solenoid model coil", Superconductor Science and Technology, vol. 22, no. 8, Art. ID 085006, 2009.

[107] N. N. Martovetsky, W. T. Reiersen, G. R. Murdoch, P. Bruzzone, and B. Stepanov, "Qualification of the US Conductors for ITER TF Magnet System", IEEE Transactions on Plasma Science, vol. 46, no. 5, pp. 1477-1483, 2018.

[108] D. C. van der Laan, P. D. Noyes, G. Miller, H. W. Weijers, and G. Willering, "Characterization of a high-temperature superconducting conductor on round core cables in magnetic fields up to 20 T", Superconductor Science and Technology, vol. 26, no. 4, Art. ID 045005, 2013.

[109] D. Uglietti, N. Bykovsky, R. Wesche, and P. Bruzzone, "Development of HTS conductors for fusion magnets", IEEE Transactions on Applied Superconductivity, vol. 25, no. 3, pp. 1-6, 2015. 
[110] M. Breschi, M. Bianchi, A. Ricchiuto, P. Ribani, and A. Devred, "Analysis of AC Losses in a CS Conductor Sample for the ITER Project", IEEE Transactions on Applied Superconductivity, vol. 28, no. 3, pp. 1-5, 2018.

[111] P. Bruzzone, M. Bagnasco, M. Calvi, F. Cau, D. Ciazynski, A. Della Corte, A. Di Zenobio, L. Muzzi, A. Nijhuis, E. Salpietro, et al., "Test results of two European ITER TF conductor samples in SULTAN", IEEE Transactions on Applied Superconductivity, vol. 18, no. 2, pp. 1088-1091, 2008.

[112] Y. A. Ilyin, A. Nijhuis, W. Abbas, P. Bruzzone, B. Stepanov, L. Muzzi, P. Gislon, and L. Zani, "Effect of cyclic loading and conductor layout on contact resistance of full-size ITER PFCI conductors", IEEE Transactions on Applied Superconductivity, vol. 15, no. 2, pp. 1359-1362, 2005.

[113] D. van der Laan and J. Weiss, "Personal Correspondence", 2018.

[114] M. Mentink, A. Dudarev, H. F. P. Da Silva, C. P. Berriaud, G. Rolando, R. Pots, B. Cure, A. Gaddi, V. Klyukhin, H. Gerwig, et al., "Design of a 56-GJ Twin solenoid and dipoles detector magnet system for the future circular collider", IEEE Transactions on Applied Superconductivity, vol. 26, no. 3, pp. 1-6, 2016.

[115] M. Mentink, H. P. Da Silva, A. Dudarev, G. Rolando, B. Cure, A. Gaddi, V. Klyukhin, H. Gerwig, U. Wagner, and H. H. J. ten Kate, "Design of 4 Tm Forward Dipoles for the FCC-hh Detector Magnet System", IEEE Transactions on Applied Superconductivity, vol. 27, no. 4, pp. 1-6, 2017. 


\section{Summary}

ReBCO coated conductors are ceramic flat tape high-temperature superconductors capable of carrying large currents in a broad temperature and magnetic field range. ReBCO tapes significantly exceed the performance reach of $\mathrm{NbTi}_{\text {and }} \mathrm{Nb}_{3} \mathrm{Sn}$ low temperature superconductors, which allows the creation of large-scale magnets capable of generating magnetic field far beyond $20 \mathrm{~T}$ at $4.5 \mathrm{~K}$ and of magnets operating in the temperature range of 30 to $60 \mathrm{~K}$, a range served uniquely by ReBCO material. Large-scale magnets require currents far beyond the capacity of a single ReBCO tape and therefore multiple ReBCO tapes must be combined into a multi-tape, high-current ReBCO cable. The three main cabling concepts today are Roebel, Twisted Stack Tape and Conductor On Round Core (CORC). The focus of the work presented in this thesis is on the development of CORC technology, which includes both the CORC conductor and its joint terminals. CORC cables are round and comprise many layers of $\mathrm{ReBCO}$ tapes wound around a central metal core. The cable is relatively flexible and allows omni-directional bending due to its round shape. CORC can be grouped in three categories: CORC cable, which is a standalone general purpose HTS cable; thin CORC wire that aims at a high-current density for application in accelerator magnets and insert coils; and CORC Cable-In-Conduit Conductor (CICC) designed for enabling stable, high-current magnets and their bus lines.

Well performing electrical connection to the CORC conductor are essential for its performance, as they have a dominant role in determining current distribution among ReBCO tapes and are a constant source of ohmic heating. Therefore, low-resistive joints that feature a uniform current distribution are key. The state-of-the art joints for CORC that were available at the start of this work required improvements, as they were not able to provide a low-resistance connection in a compact manner with uniform current injection and extraction. A novel conductor joint design was realized that offers a significant improvement of current distribution among ReBCO tapes and a significant reduction in joint resistance compared to previous designs. This new design introduces staging, also known as tapering, to the layers of the CORC cable, to form a staircase-like geometry at the cable ends. In this design, current is able to flow directly to the more inner situated layers, without having to pass the highly resistive substrate and buffer layers of the tapes that were in the layers above. A joint preparation method was developed featuring a practical realization of the new design. Moreover, a numerical model was developed to simulate and predict the performance of the new and old joint terminals. Several demonstration joints were prepared to test and to fine-tune the preparation procedures. All CORC samples tested throughout this work included joint terminals of the new design. 
CORC conductors and their production technique were new and in the early stage of development at the start of this work. A better understanding of the performance of CORC conductors, current sharing between layers, current distribution between individual ReBCO coated conductors, CORC bending/handling and all details appertained to the production of CORC conductors are required. Therefore, methods for predicting the performance of CORC conductors were developed using techniques to scale the performance of single ReBCO tapes at $77 \mathrm{~K}$ to the performance of a CORC wire or cable at $4.2 \mathrm{~K}$ in external magnetic field. A first $12 \mathrm{~m}$ long CORC cable with $38 \mathrm{ReBCO}$ tapes in 12 layers was procured by CERN. Part of this cable is characterized at 1.9 and $4.2 \mathrm{~K}$ in a magnetic field up to $9.6 \mathrm{~T}$ to investigate scaling methods and its joint terminals prepared according to the new joint design. This cable was later used in the first-in-the-world CORC six-around-one Cable-In-Conduit Conductor developed within this project.

Thinner substrate ReBCO tapes make thin CORC wires of 3 to $4 \mathrm{~mm}$ diameter possible. The reduction of the tape's substrate thickness from 100 via 50 to 30 micron leads to a significantly reduced minimum bending radius of $R e B C O$ tapes and cables made out of these. The 30 micron substrate tapes can now also be manufactured with a width of only $2 \mathrm{~mm}$. Thinner substrates and narrower tapes allow production of CORC wires with more flexibility and a much higher current density compared to the thicker, 5 to $8 \mathrm{~mm}$ in diameter, CORC cables. CORC wires with various tape layouts were tested in common effort of CERN, ACT and the University of Twente to advance this breakthrough technology. Four CORC wires, of 3.0 to $4.5 \mathrm{~mm}$ diameter, were tested in transverse magnetic field of up to $11 \mathrm{~T}$ as small solenoids at $4.2 \mathrm{~K}$ and in self field at $77 \mathrm{~K}$. The tests demonstrate the ease of use and high performance of the new generation of CORC wires. In addition the tests provide useful feedback for optimizing wire manufacturing and joint terminal production.

The ReBCO CORC six-around-one Cable-In-Conduit Conductor is a high-current multistrand conductor aimed at application in large-scale magnets, i.e. for detector- and fusion experiments, but also for bus lines that feed high currents to such magnets. Three firstin-the-world CORC Cable-In-Conduit Conductors were prepared at CERN. The very first CICC is $1.7 \mathrm{~m}$ long and has an aluminium alloy jacket. It has a design current of $48 \mathrm{kA}$ at $10 \mathrm{~T}$ and $4.2 \mathrm{~K}$ and $13 \mathrm{kA}$ at $77 \mathrm{~K}$ in self-field and was successfully tested at CERN. The conductor's performance was according to prediction and showed that the preparation and prediction methods are valid. The second and third CIC-Conductor were especially designed for application in fusion and detector magnets. The second conductor has a copper jacket that allows conduction cooling, which is the more practical cooling solution for large magnets without large heat loads. The third CIC-Conductor was designed for fusion magnets and comprised a stainless steel jacket and internal forced-flow cooling, a requirement for the large heat loads common to fusion type of magnets. The six-aroundone cable bundles in both jackets were both rated for $80 \mathrm{kA}$ at $12 \mathrm{~T}$.

Conductors two and three were tested at the SULTAN test facility of PSI, Villigen, Switzerland in a temperature range of 5 to $60 \mathrm{~K}$ in magnetic field up to $11 \mathrm{~T}$. The CORC CICC with the stainless steel jacket for fusion magnets performed as expected in the high temperature regime of 40 to $60 \mathrm{~K}$. The CORC CICC with the copper jacket for detector magnets, however, performed below expectation and demonstrated a critical current of $30 \%$ to 
$40 \%$ of the expected value. The limited performance of this CICC prevented accurate measurements of the other CICC in the temperature range of 5 to $40 \mathrm{~K}$ as both conductors were tested in series. Both, forced-flow and conduction cooling, proved to be effective cooling methods for the CORC Cable-In-Conduit Conductor. The strands from the CICC with copper jacket were extracted and tested individually to localize and quantify degradation in each CORC strand. Degradation appeared to have occurred only in the high-field region, indicating that the large electro-magnetic load locally pushed the strain in the ReBCO tapes over their intrinsic limit. No clear indication was observed that a similar type of degradation occurred in the CORC CICC with stainless steel jacket. The cause of the degradation was traced back to a cabling parameter unique for the CORC strands in the CORC CICC with copper jacket.

The results achieved with the three unique ReBCO tape based Cable-In-Conduit Conductors clearly demonstrate that exercising conductor production is extremely useful in order to identify relevant design and production parameters for controlling the quality of these new-technology conductors. 


\section{Samenvatting}

ReBCO coated conductors zijn platte ceramische hoge-temperature supergeleiders die in staat zijn om hoge stromen te dragen in een breed temperatuur en magneetveld bereik. ReBCO tapes overtreffen de prestaties van $\mathrm{NbTi}$ en $\mathrm{Nb}_{3} \mathrm{Sn}$ lage-temperatuur supergeleiders aanzienlijk, waardoor het mogelijk wordt grootschalige magneten te maken die een magnetisch veld van meer dan $20 \mathrm{~T}$ bij $4.5 \mathrm{~K}$ kunnen genereren en magneten die werken in het temperatuurbereik van 30 tot $60 \mathrm{~K}$, een bereik uniek voor ReBCO materiaal. Grootschalige magneten vereisen stromen die de capaciteit van een enkele ReBCO tape ver overtreffen en daarom moeten meerdere tapes worden gecombineerd in een multi-tape, hoge-stroom ReBCO kabel. De drie belangrijkste kabel-concepten momenteel zijn Roebel, Twisted Stack Tape en Conductor On Round Core (CORC). De focus van het werk in dit proefschrift ligt op de ontwikkeling van de CORC-technologie, zowel de CORC-geleider als de electrische connecties naar deze geleider. CORC kabels zijn rond en bestaan uit vele lagen $R e B C O$ tapes die rond een centrale metalen kern zijn gewonden. De kabel is relatief flexibel en kan alle kanten opbuigen vanwege z'n ronde vorm. CORC kan worden gegroepeerd in drie categorieën: CORC-kabel, een op zichzelf staande universele HTS-kabel; dun CORC-draad, een draad met hoge stroomdichtheid voor toepassing in versneller-magneten; en CORC Cable-In-Conduit Conductor (CICC) ontworpen voor magneten met hoge stroom en voor de aanvoer van stroom voor zulke magneten.

Goed presterende elektrische verbindingen naar de CORC-geleider zijn essentieel, omdat ze een dominante rol spelen bij het bepalen van de stroom-verdeling tussen ReBCO tapes en een constante bron van ohmse verliezen zijn. Daarom zijn verbindingen met lage weerstand en uniforme stroomverdeling van cruciaal belang. De verbindingen voor CORC die beschikbaar waren aan het begin van dit werk vereisten een verbetering, omdat ze niet in staat waren om op een compacte manier een verbinding met lage weerstand met uniforme stroominjectie en -extractie te bieden. Daarom, is er een nieuw ontwerp van de verbinding gerealiseerd die stroomverdeling tussen $R e B C O$ tapes aanzienlijk verbetert en de weerstand vermindert in vergelijking met eerdere ontwerpen. Dit nieuwe ontwerp introduceert 'staging', ook wel 'tapering' genoemd, van de lagen van de CORC-kabel, dat een trap-achtige geometrie aan de kabeluiteinden vormt. Hierdoor kan de stroom direct naar de binnenste lagen stromen, zonder dat het het hoog-resistieve substraat en de bufferlagen van de buitenste lagen hoeft te passeren. Een methode is ontwikkeld voor de praktische realisatie van het nieuwe ontwerp. Daarnast is een numeriek model ontwikkeld om de prestaties van de nieuwe en oude verbindingen te simuleren en te voorspellen. Er zijn verschillende demonstratie-verbindingen geprepareerd om de proce- 
dures te testen en te verfijnen. Alle CORC geleiders die tijdens dit werk werden getest, omvatten verbindingen van het nieuwe ontwerp.

De CORC geleiders en hun productietechniek waren nieuw en in de vroege stadia van ontwikkeling aan het begin van dit werk. Een beter begrip van de prestaties van CORCgeleiders, de stroomverdeling tussen verschillende lagen, de stroomverdeling tussen individuele ReBCO tapes, het buigen van de kabel en alle details die betrekking tot de productie van CORC-geleiders is vereist. Methodes voor het voorspellen van de prestaties van CORC-geleiders zijn ontwikkeld die de prestatie van een enkel tape schalen naar de prestatie van een CORC geleider. De eerste $12 \mathrm{~m}$ lange CORC-kabel met 38 ReBCO tapes in 12 lagen is verkregen door CERN. Een deel van deze kabel is gekarakteriseerd op 1.9 en $4.2 \mathrm{~K}$ in een magnetisch veld tot $9.6 \mathrm{~T}$ om de schalings-methodes en de verbindingen, die zijn vervaardigd volgens het nieuwe ontwerp, te testen. Deze kabel is later gebruikt in de eerste CORC Cable-In-Conduit Conductor ter wereld die binnen dit project is ontwikkeld.

ReBCO tapes met dunner substraat maken dunne CORC-draden met een diameter van 3 tot $4 \mathrm{~mm}$ mogelijk. De reductie van de substraatdikte van 100 via 50 tot 30 micron leidt tot een aanzienlijk verminderde minimale buigradius van de tapes en de kabels die hiervan gemaakt zijn. De tapes met 30 micron substraat kunnen worden vervaardigd met een breedte van slechts $2 \mathrm{~mm}$. Dunnere substraten en smallere tapes maken de productie van CORC-draden mogelijk met meer flexibiliteit en een veel hogere stroomdichtheid in vergelijking met de dikkere CORC-kabels met een diameter van 5 tot $8 \mathrm{~mm}$. CORCdraden met verschillende tape layouts zijn in gezamenlijke inspanning van CERN, ACT en de Universiteit Twente getest om deze technologie te bevorderen. Vier CORC-draden, met een diameter van 3.0 tot $4.5 \mathrm{~mm}$, zijn getest in een transversaal magnetisch veld tot $11 \mathrm{~T}$ en een temperatuur van $4.2 \mathrm{~K}$ als kleine spoelen. De tests demonstreren het gebruiksgemak en de hoge prestaties van de nieuwe generatie CORC-draden. Bovendien bieden de tests bruikbare terugkoppeling voor het optimaliseren van de draad-productie en de electrische verbindingen.

De ReBCO CORC zes-rond-één Cable-In-Conduit Conductor is een hoge-stroom multidraad geleider gericht op toepassing in grootschalige magneten, voor onder andere detector en fusie-experimenten, maar ook voor aanvoers-lijnen die hoge stromen naar dergelijke magneten voeden. Drie eerste-in-de-wereld CORC CIC-geleiders zijn ontwikkeld op CERN. De allereerste CICC is $1.7 \mathrm{~m}$ lang en heeft een behuizing van aluminium legering. De geleider heeft een ontwerpstroom van $48 \mathrm{kA}$ bij $10 \mathrm{~T}$ en $4.2 \mathrm{~K}$ en $13 \mathrm{kA}$ bij $77 \mathrm{~K}$ in eigen magneetveld en is met succes getest op CERN. De prestaties van de geleider waren volgens de voorspelling en toonden aan dat de preparatie- en voorspellings methoden naar behoren werken. De tweede en derde CIC-geleiders zijn speciaal ontworpen voor toepassing in detector- en fusie-magneten. De tweede geleider heeft een koperen behuizing die koeling via geleiding mogelijk maakt, wat een praktischere koel-oplossing is voor grote magneten zonder grote warmtebelasting. De derde CIC-geleider is ontworpen voor fusie-magneten en bestaat uit een roestvrijstalen behuizing en inwendige convectie koeling, een vereiste voor de grote warmtebelastingen in zulke type magneten. De zesrond-één kabelbundels in beide geleiders hebben een ontwerp kritieke stroom van $80 \mathrm{kA}$ bij $12 \mathrm{~T}$ en $4.5 \mathrm{~K}$. 
Geleiders twee en drie zijn getest in de SULTAN-testfaciliteit van PSI, Villigen, Zwitserland in een temperatuurbereik van 5 tot $60 \mathrm{~K}$ in een magnetisch veld tot $11 \mathrm{~T}$. De CORC CICC met de roestvrijstalen behuizing voor fusie-magneten presteerde naar verwachting in de hoge temperatuurregime van 40 tot $60 \mathrm{~K}$. De CORC CICC met de koperen behuizing voor detector-magneten presteerde echter onder verwachting en toonde een kritische stroom van $30 \%$ tot $40 \%$ van de verwachte waarde. De beperkte prestaties van deze CICC beperkte metingen van de andere CICC in het temperatuurbereik van 5 tot $40 \mathrm{~K}$, omdat beide geleiders in serie zijn getest. Zowel convectie als geleidings koeling bleken effectieve koelmethodes voor de CORC CICC. De CORC-draden van de CICC met koperen behuizing zijn afzonderlijk getest om degradatie in elke CORC-draad te lokaliseren en te kwantificeren. Degradatie bleek alleen in het hoogveldgebied te hebben plaatsgevonden, wat aangeeft dat de grote elektromagnetische belasting lokaal stress in de ReBCO tapes over hun intrinsieke limiet heeft geduwd. Geen duidelijke aanwijzingen zijn gevonden dat een vergelijkbaar type degradatie optrad in de CORC CICC met roestvrijstalen behuizing. De oorzaak van de degradatie is terug te leiden naar een bekabelingsparameter die uniek is voor de CORC-draden in de CORC CICC met koperen behuizing.

De behaalde resultaten met de drie unieke ReBCO CORC Cable-In-Conduit Conductors tonen duidelijk aan dat oefenen met het prepareren van de geleider uiterst nuttig is om relevante ontwerp- en productieparameters te identificeren voor het beheer van de kwaliteit van deze nieuwe geleider technologie. 


\section{Acknowledgments}

During my masters degree in Applied Physics, I did my internship at CERN and really enjoyed the working environment, the drive to develop new technologies and of course the beautiful mountainous surroundings. Therefore, near the end of my master studies, I asked my internship supervisor Herman ten Kate if there was any possibility for me to continue in the field of superconductivity at CERN. I was offered a PhD position on the topic of advancing the CORC technology, which was the start of 4.5 years of interesting, intensive, sometimes frustrating, but always satisfying work. I would like to offer my sincere gratitude to my promoter Herman ten Kate, co-promoter Marc Dhallé and daily supervisor at CERN Alexey Dudarev for this amazing opportunity, all of their guidance and the interesting discussions.

The work contained a large portion of experimental work and all the work could not have been completed without the aid and expertise of skilled technical staff Nikolay, Philippe, Igor and Anton at CERN and Sander and Peng at the University of Twente, who helped in the preparation of the CORC conductors and the experimental setups. The practical realization of the samples did not always go smoothly, but with all their help everything always worked out in the end.

Danko and Jeremy, of Advanced Conductor Technologies, deserve a word of gratitude. Not only have they prepared all the CORC wires, cables and strands, they also participated in a lot of fruitful discussions and and provided a lot of help with writing several papers.

The first three-and-a-half years of my PhD, I was at the CERN. There I met a lot of great people and I want to thank them all for their discussions and help during work, but also for the fun times at dinner parties, game nights, amazing hikes and challenging via-ferrata climbs. Thanks Helder, Matthijs, Nikkie, Jeroen, Felix, Jaakko, Valentina, Erwin, Rosalinde and all others!

The last part of my PhD I finished at the University of Twente. Although I have been away from Enschede for over 3 years, a lot a familiar faces remained both at the EMS group of the university, as well as at my old sports club Arashi. I want to thank you all for the great time during lunch breaks, during sports and all other activities!

I would like to give a special thanks to Tiemo. It is always nice talking to you, as you always have so many innovative ideas. I will for sure come and visit you in Germany. And a special thanks to Bas, who was always happy to talk, even when times were tough, and I wish him all the best for the many years to come. 
Ik wil graag mijn moeder en de rest van de familie bedanken voor al hun hulp en steun in de afgelopen jaren! En in het bijzonder mijn vader, die dit zeker mee had willen maken en heel erg trots zou zijn geweest.

And of course, I would like to thank Anne, who always stood by my side through the difficulty of being so far away from each other for most of the time. During first three years of my $\mathrm{PhD}$, we saw each other most often when we traveled together to many remote places all around Europe, North- and Central America and Asia and I hope in the future more beautiful destinations are added to our list. The last year we have been living together and I really appreciate your incredible patience with me. 


\section{List of Publications}

T. Mulder, J. Weiss, D. van der Laan, M. Dhallé and H.H.J. ten Kate, 'Development of ReBCO-CORC Wires with Current Densities of 400 to $600 \mathrm{~A} / \mathrm{mm}^{2}$ at $10 \mathrm{~T}$ and $4.2 \mathrm{~K}$, IEEE Transactions on Applied Superconductivity, vol. 28, no. 3, Art. ID 4800504, 2018.

T. Mulder, D. van der Laan, J. Weiss, A. Dudarev, M. Dhallé and H.H.J ten Kate, 'Design and Preparation of Two ReBCO-CORC Cable-In-Conduit Conductors for Fusion and Detector Magnets', IOP Conference Series: Materials Science and Engineering, vol. 279, no. 1, Art. ID 012033, 2017.

T. Mulder, J. Fleiter, G. Willering, A. Dudarev, M. Mentink, M. Dhallé and H.H.J. ten Kate, 'Demonstration of the ReBCO CORC 47kA @ 10T/4.2 K Cable-In-Conduit-Conductor and its Joint Terminals at 4.5 and 77 K', IEEE Transactions on Applied Superconductivity, vol. 27, no. 4, Art. ID 4801004, 2017.

T. Mulder, A. Dudarev, M. Mentink, H. Silva, D. van der Laan, M. Dhallé and H.H.J. ten Kate, 'Design and Manufacturing of a $45 \mathrm{kA}$ at 10 T ReBCO-CORC Cable-In-Conduit Conductor for Large-Scale Magnets', IEEE Transactions on Applied Superconductivity, vol. 26, no. 4, Art. ID 4803605, 2016.

T. Mulder, A. Dudarev, M. Mentink, D. van der Laan, M. Dhallé and H.H.J. ten Kate, 'Performance Test of an 8 kA@ 10-T 4.2-K ReBCO-CORC Cable', IEEE Transactions on Applied Superconductivity, vol. 26, no. 4, Art ID 4803705, 2016.

T. Mulder, A. Dudarev, M. Mentink, M. Dhallé and H.H.J. ten Kate, 'Development of Joint Terminals for a New Six-Around-One ReBCO-CORC Cable-In-Conduit Conductor rated $45 \mathrm{kA}$ at $10 \mathrm{~T} / 4$ K'. IEEE Transactions on Applied Superconductivity, vol. 26, no. 3, Art ID 4801704, 2016.

T. Mulder, A. Dudarev, D. van der Laan, M. Mentink, M. Dhallé and H.H.J. ten Kate, 'Optimized and Practical Electrical Joints for CORC type HTS Cables', IOP Conference Series: Materials Science and Engineering, vol. 102, no. 1, Art ID 012026, 2015. 

